Prepared for the U.S. Department of Energy

under Contract DE-AC05-76RL01830

\title{
Coupled Multi-Group Neutron Photon Transport for the Simulation of High-Resolution Gamma-Ray Spectroscopy Applications
}

KA Burns

August 2009

\section{Pacific Northwest}

NATIONAL LABORATORY

Proudly Operated by Battelle Since 1965 


\title{
DISCLAIMER
}

This report was prepared as an account of work sponsored by an agency of the United States Government. Neither the United States Government nor any agency thereof, nor Battelle Memorial Institute, nor any of their employees, makes any warranty, express or implied, or assumes any legal liability or responsibility for the accuracy, completeness, or usefulness of any information, apparatus, product, or process disclosed, or represents that its use would not infringe privately owned rights. Reference herein to any specific commercial product, process, or service by trade name, trademark, manufacturer, or otherwise does not necessarily constitute or imply its endorsement, recommendation, or favoring by the United States Government or any agency thereof, or Battelle Memorial Institute. The views and opinions of authors expressed herein do not necessarily state or reflect those of the United States Government or any agency thereof.

\author{
PACIFIC NORTHWEST NATIONAL LABORATORY \\ operated by \\ BATTELLE \\ for the \\ UNITED STATES DEPARTMENT OF ENERGY \\ under Contract DE-AC05-76RL01830
}

Printed in the United States of America
Available to DOE and DOE contractors from the Office of Scientific and Technical Information,
P.O. Box 62, Oak Ridge, TN 37831-0062;
ph: (865) 576-8401
fax: $(865)$ 576-5728
email: reports@adonis.osti.gov

\begin{abstract}
Available to the public from the National Technical Information Service, U.S. Department of Commerce, 5285 Port Royal Rd., Springfield, VA 22161 ph: (800) 553-6847 fax: $(703) 605-6900$ email: orders@ntis.fedworld.gov online ordering: http://www.ntis.gov/ordering.htm
\end{abstract}

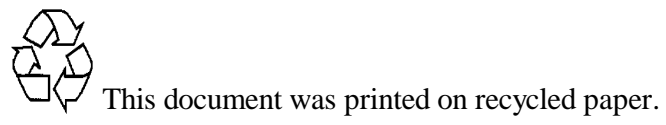




\title{
Coupled Multi-Group Neutron Photon Transport for the SiMULATION OF HIGH-RESOLUTION GAMMA-RAY SPECTROSCOPY APPLiCATIONS
}

\author{
A Dissertation \\ Presented to \\ The Academic Faculty \\ By \\ Kimberly Ann Burns \\ In Partial Fulfillment \\ of the Requirements for the Degree \\ Doctoral of Philosophy in \\ Nuclear Engineering
}

Georgia Institute of Technology

August 2009 


\section{Coupled Multi-Group Neutron Photon Transport for the SIMULATION OF HIGH-RESOLUTION GAMMA-RAY SPECTROSCOPY APPLiCATIONS}

Approved by:

Dr. Nolan E. Hertel, Advisor Nuclear and Radiological Engineering Georgia Institute of Technology

Dr. Farzad Rahnema

Nuclear and Radiological Engineering Georgia Institute of Technology

Dr. Chris Wang

Nuclear and Radiological Engineering Georgia Institute of Technology

Dr. Bojan Petrovic

Nuclear and Radiological Engineering Georgia Institute of Technology
Dr. L. Eric Smith

Radiation Detection and Nuclear Sciences Pacific Northwest National Laboratory

Dr. Richard Pagh

Nuclear Materials and Engineering Analysis

Pacific Northwest National Laboratory

Dr. Eva Lee

Industrial and Systems Engineering Georgia Institute of Technology

Dr. William David Kulp III

Physics

Georgia Institute of Technology

Date Approved:

June 12, 2009 


\section{ACKNOWLEDGEMENTS}

First and foremost, I would like to thank my family. Since I was a little girl, you have always told me I can be anything I want. Your faith in me and the belief that I can achieve anything by setting goals for myself has helped me reach this point in my life. I would not be here if not for your support. Thank you.

I would like to thank the entire RADSAT team - Eric Smith, Christopher Gesh, Michael Batdorf, Mark Shaver, Edward Ellis, Erin Miller, Andrei Valsan, Richard Wittman, Jacob Benz and Andrew Casella, particularly Eric Smith, for entrusting such a large section of the project to me. The aid and support of the team has helped to guide and shape the task at hand. The collaboration with the RADSAT team has made working on this project an enjoyable task. I would also like to give a special thanks to Michael Batdorf for helping me turn my algorithms for the cross section collapsing into a fully functional code. He is responsible for all of the computer programming implemented in conjunction with this work. Without his help, the cross section development would not have reached the maturity it has.

Thank you to my committee - Dr. Nolan Hertel, Dr. Eric Smith, Dr. Richard Pagh, Dr. Farzad Rahnema, Dr. Chris Wang, Dr. Eva Lee, Dr. Bojan Petrovic, and Dr. William David Kulp - for their time and contributions. I would particularly like to thank Dr. Nolan Hertel, my advisor, for his support and guidance. Eric Smith and Richard Pagh also deserve special thanks for their contributions to the project and for their patience with me. 
I would also like to acknowledge a few people who helped me with some technical issues and thank them for their individual contributions. Ian Davis of Transpire, Inc. and Christopher Gesh of Pacific Northwest National Laboratory provided guidance for the application of Attila. John Mattingly of Sandia National Laboratories provided the Kynea3 cross-section library. A.C. Kahler and Morgan White of Los Alamos National Laboratory answered a number of questions on NJOY. Jeffrey Favorite, also of Los Alamos National Laboratory, converted the Kynea3 cross-section library to a format compatible with Attila. Gus Caffrey of Idaho National Laboratory and the United States Army provided some funding and motivation for this project through the PINS (Portable Isotopic Neutron Spectroscopy) Project. Thank you to everyone. I could not have done this without everyone's help and support. 


\section{TABLE OF CONTENTS}

Page

ACKNOWLEDGEMENTS

iii

LIST OF TABLES

viii

LIST OF FIGURES

$\mathrm{X}$

LIST OF SYMBOLS X xiii

LIST OF ABBREVIATIONS $\quad$ Xv

$\begin{array}{ll}\text { SUMMARY Xvi } & \text { XVi }\end{array}$

CHAPTER 1 BACKGROUND 1

1.1 Computational Methods 2

1.1.1 Monte Carlo Methods 2

1.1.2 Deterministic Methods 5

1.2 Coupled Stochastic-Deterministic Methods $\quad 9$

1.3 Applications of Coupled Method 14

1.3.1 Interrogation of Cargo Containers 14

$\begin{array}{lll}\text { 1.3.2 } \mathrm{UF}_{6} & 16\end{array}$

1.3.3 Prompt Gamma Neutron Activation Analysis 17

$\begin{array}{lll}\text { CHAPTER } 2 & \text { METHODS }\end{array}$

2.1 Experimental Design for Testing Methods 19

$\begin{array}{lll}2.2 & \text { Methods Discussion } & 21\end{array}$

$\begin{array}{lll}\text { 2.2.1 Neutron Transport Calculation } & 23\end{array}$

2.2.2 Generation of Neutron-Induced Photon Spectrum 44

2.2.3 Photon Transport 56

2.2.4 Detector Response Calculation $\quad 60$ 
$\begin{array}{lll}\text { CHAPTER } 3 & \text { RESULTS }\end{array}$

$\begin{array}{lll}3.1 & \text { Polyethylene } & 62\end{array}$

3.1.1 Cross Sections 63

$\begin{array}{lll}\text { 3.1.2 Detector Response } & 67\end{array}$

$\begin{array}{lll}3.2 & \text { Phosphorus } & 69\end{array}$

3.2.1 Neutron Flux Comparison $\quad 69$

$\begin{array}{lll}\text { 3.2.2 Cross Sections } & 72\end{array}$

3.2.3 Photon Flux within Detector 75

$\begin{array}{lll}3.3 & \text { Nitrogen } & 77\end{array}$

$\begin{array}{lll}\text { 3.3.1 Neutron Flux Comparison } & 77\end{array}$

$\begin{array}{lll}\text { 3.3.2 Cross Sections } & 79\end{array}$

3.3.3 Photon Flux within Detector 84

$\begin{array}{llr}3.4 & \text { Oxygen } & 85\end{array}$

3.4.1 Neutron Flux Comparison $\quad 86$

$\begin{array}{lll}\text { 3.4.2 Cross Sections } & 88\end{array}$

3.4.3 Photon Flux within Detector 92

$\begin{array}{lll}3.5 & \text { Iron } & 94\end{array}$

3.5.1 Neutron Flux Comparison 94

$\begin{array}{lll}\text { 3.5.2 Cross Sections } & 95\end{array}$

3.5.3 Photon Flux within Detector 102

$\begin{array}{lll}\text { CHAPTER } 4 \text { CONCLUSIONS } & 105\end{array}$

$\begin{array}{lll}\text { CHAPTER } 5 & \text { RECOMMENDATIONS } & 108\end{array}$

APPENDIX A Example Attila Input File for Neutron Transport Problem in $\mathrm{C}_{2} \mathrm{H}_{4}$

APPENDIX B Radion15, SCALE44 and Kynea3 Group Structure 119

APPENDIX C MCNP5 Input File for $\mathrm{C}_{2} \mathrm{H}_{4}$ Cube 122 
APPENDIX D Summary of NJOY99.259 Modules Used in Calculations

APPENDIX E NJOY99.259 Input File to Produce ACER file for Hydrogen

APPENDIX F RADSAT-NG Cross Section Example Format

APPENDIX G NJOY99.259 Input File to Produce GROUPR file for Hydrogen

133

APPENDIX H Example RADSAT "Pseudo-Isotope" File for Phosphorus

REFERENCES 


\section{LIST OF TABLES}

Table 1. Comparison of MCNP and RADSAT Computational Times for Simple Cube Problems xix

Table 2. Reaction Rates Calculated using Attila Flux and Kynea3, SCALE44 and Radion 15 Cross Sections and Compared to Reaction Rates Calculated Using MCNP.

Table 3. Comparison of the Scalar Flux $\left(\gamma / \mathrm{cm}^{2}\right.$-sec-source particle) in Each Photopeak inside the Detector from the Irradiation of Polyethylene.

Table 4. Comparison of Total Cross Section for RADSAT-NG and Cross Sections

Produced by GROUPR for Hydrogen, Deuterium and Carbon.....

Table 5. Comparison of Attila and MCNP Total Neutron Flux for the Irradiation of Phosphorus

Table 6. Discrete Gamma Rays Produced by ${ }^{31} \mathrm{P}$

Table 7. Comparison of Total Cross Section for RADSAT-NG and Cross Sections

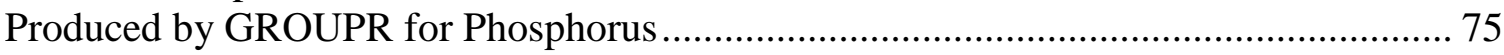

Table 8. Comparison of Photon Flux for Top 2 Most Intense Peaks Produced by the Neutron Irradiation of Phosphorus.

Table 9. Comparison of Attila and MCNP Total Neutron Flux for the Irradiation of Nitrogen

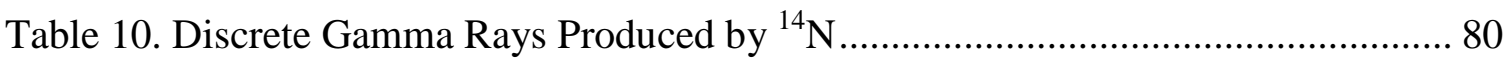

Table 11. Comparison of Total Cross Section for RADSAT-NG and Cross Sections Produced by GROUPR for ${ }^{14} \mathrm{~N}$........................................................... 83

Table 12. Comparison of Photon Flux for Top 5 Most Intense Peaks Produced by the Neutron Irradiation of Nitrogen

Table 13. Comparison of Attila and MCNP Total Neutron Flux for the Irradiation of Oxygen.

Table 14. Discrete Gamma Rays Produced by ${ }^{16} \mathrm{O}$ 88 
Table 15. Comparison of Total Cross Section for RADSAT-NG and Cross

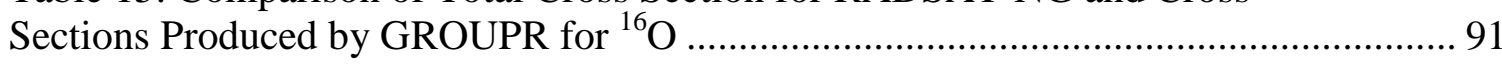

Table 16. Comparison of Photon Flux for Top 6 Most Intense Peaks Produced by the Neutron Irradiation of Oxygen...................................................................... 93

Table 17. Comparison of Attila and MCNP Total Neutron Flux for the Irradiation

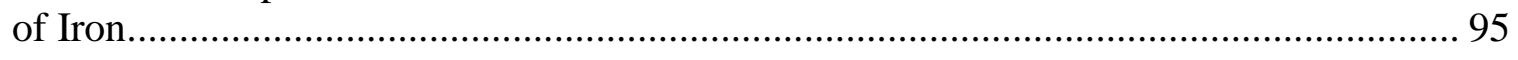

Table 18. Discrete Gamma Rays Produced by Iron..................................................... 97

Table 19. Comparison of Total Cross Section for RADSAT-NG and Cross

Sections Produced by GROUPR for Iron ........................................................... 101

Table 20. Comparison of Photon Flux for Top Seven Most Intense Peaks

Produced by the Neutron Irradiation of Iron........................................................... 104

Table 21. Comparison of MCNP and RADSAT Computational Times for Simple

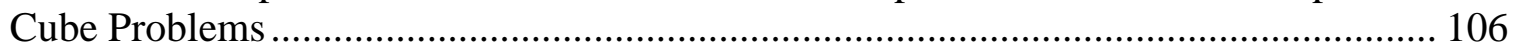

Table 22. Comparison of Radion15, SCALE44 and Kynea3 Neutron Energy

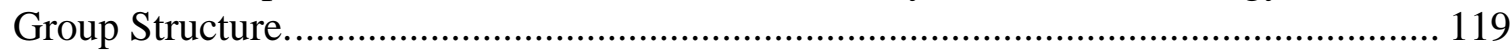




\section{LIST OF FIGURES}

Page

Figure 1: Example of Ray Effects Resulting from the Calculation of the Scalar Flux for a Point Source in Air. $S_{8}, S_{16}$, and $S_{32}$ represent the $S_{n}$ order used in the discrete ordinates calculation.

Figure 2: Illustration of last-collided flux (LCF) edit as a means to mitigate ray effects in discrete ordinates solutions [1]. 12

Figure 3. Passive Interrogation of Cargo Container Using Gamma Detectors to Find Gamma Rays Produced by Neutron Interactions in the Container. 15

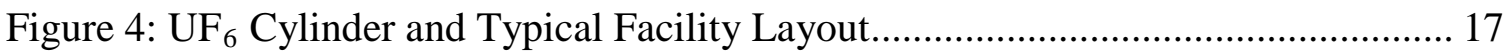

Figure 5. Prompt Gamma Neutron Activation Analysis Setup..................................... 18

Figure 6. Source-sample-detector geometry for the neutron activation analysis problems used in code-to-code comparisons of RADSAT and MCNP5. 20

Figure 7.Schematic of the Ortec Detective HPGe. (See text for a color-coded description of the detector region)

Figure 8. Procedure for Calculating Coupled Neutron-Photon Problems Using RADSAT. The blue boxes (neutron transport, photon transport and detector response) apply principles previously included in RADSAT. Yellow boxes (RADSAT-NG cross-section preparation and neutron-induced photon source generation using RADSAT) are unique steps developed in this work for coupled neutron-photon problems.

Figure 9. Comparison of Volume-Averaged Neutron Flux in Polyethylene Cube from MCNP and Attila using Radion15, SCALE44 and Kynea3 Cross-Section Libraries

Figure 10 Comparison of Volume-Averaged Thermal Neutron Flux in Polyethylene Cube from MCNP and Attila using Radion15, SCALE44 and Kynea3 Cross-Section Libraries

Figure 11. Carbon Radiative Capture Photon Production Cross Section in "Continuous Energy", Kynea3 Group Structure, SCALE 44 Group Structure and Radion15 Group Structure 
Figure 12. Carbon Inelastic Scattering Photon Production Cross Section in "Continuous Energy", Kynea3 Group Structure, SCALE 44 Group Structure and Radion15 Group Structure

Figure 13. TecPlot of Slices of the Log of the Scalar Flux within the Polyethylene Cube. A is a slice along the $\mathrm{z}$-axis at $\mathrm{z}=0, \mathrm{~B}$ is a slice along the $\mathrm{x}$-axis at $\mathrm{x}=0$ and $\mathrm{C}$ is a slice along the $\mathrm{x}$-axis at $\mathrm{x}=9$.

Figure 14. Comparison of Volume-Averaged Photon Flux in Detector for Uniformly Distributed Induced Photon Spectrum, Spatially Dependent Induced Photon Spectrum and MCNP 35

Figure 15. TecPlot of the Scalar Photon Flux within the Polyethylene Cube. 37

Figure 16. Adaptive Algorithm Applied to Neutron-Induced Photon Spectrum from the Irradiation of Iron

Figure 17. Comparison of Hydrogen Radiative Capture Photon Production Cross Section for RADSAT-NG and GROUPR.

Figure 18. Comparison of Deuterium Radiative Capture Photon Production Cross Section for RADSAT-NG and GROUPR

Figure 19. Comparison of Carbon Radiative Capture Photon Production Cross

Section for RADSAT-NG and GROUPR.

Figure 20. Comparison of Carbon Inelastic Scatter Photon Production Cross

Section for RADSAT-NG and GROUPR..

Figure 21. RADSAT and MCNP Detector Response for Neutron Irradiated Polyethylene.

Figure 22. Comparison of Attila and MCNP Neutron Flux for the Irradiation of Phosphorus

Figure 23. Comparison of Phosphorus Radiative Capture Photon Production Cross Section for RADSAT-NG and GROUPR.

Figure 24. Comparison of Phosphorus "Nonelastic" Photon Production Cross Section for RADSAT-NG and GROUPR

Figure 25. Code-to-Code Comparison Using RADSAT and MCNP5 of VolumeAveraged Flux at the Detector Location for Neutron Activation Analysis of Phosphorus. 76

Figure 26. Comparison of Attila and MCNP Neutron Flux for the Irradiation of Nitrogen 
Figure 27. Comparison of ${ }^{14} \mathrm{~N}$ Radiative Capture Photon Production Cross Section for RADSAT-NG and GROUPR 80

Figure 28. Comparison of ${ }^{15} \mathrm{~N}$ (n,np) Photon Production Cross Section for RADSAT-NG and GROUPR 81

Figure 29. Comparison of Energy-dependent ${ }^{14} \mathrm{~N}$ Radiative Capture Photon

Production Cross Section for RADSAT-NG, GROUPR and ACER 82

Figure 30. Comparison of Volume-Averaged Photon Flux in Detector using RADSAT and MCNP for Nitrogen. 85

Figure 31. Comparison of Attila and MCNP Neutron Flux for the Irradiation of Oxygen.....

Figure 32. Comparison of the GROUPR and RADSAT-NG ${ }^{16} \mathrm{O}$ Radiative Capture Photon Production Cross Section.

Figure 33. Comparison of the GROUPR and RADSAT-NG ${ }^{16} \mathrm{O}(\mathrm{n}, \alpha)$ Photon

Production Cross Section.... 89

Figure 34. Comparison of Energy-dependent ${ }^{16} \mathrm{O}(\mathrm{n}, 2 \alpha)$ Photon Production Cross Section for RADSAT-NG, GROUPR and ACER

Figure 35. Comparison of Volume-Averaged Photon Flux in Detector using RADSAT and MCNP for Oxygen

Figure 36. Comparison of Attila and MCNP Neutron Flux for the Irradiation of Iron .

Figure 37. Comparison of the GROUPR and RADSAT-NG ${ }^{54} \mathrm{Fe}$ Radiative Capture Photon Production Cross Section.

Figure 38. Comparison of the GROUPR and RADSAT-NG ${ }^{56} \mathrm{Fe}$ Radiative Capture Photon Production Cross Section

Figure 39. Comparison of the GROUPR and RADSAT-NG ${ }^{58} \mathrm{Fe}$ Radiative Capture Photon Production Cross Section

Figure 40. Comparison of the GROUPR and RADSAT-NG ${ }^{56} \mathrm{Fe}$ (n,p) Photon Production Cross Section

Figure 41. Comparison of Volume-Averaged Photon Flux in Detector using RADSAT and MCNP for Iron 104 


\section{LIST OF SYMBOLS}
$\sigma_{0}^{i}$
Background Cross Section
$\phi^{i}(E)$
Bondarenko Approximated Scalar Flux for Nuclide i
$\phi_{i}$
Cell Averaged Flux
$f\left(E_{n} \rightarrow E_{\gamma}\right)$
Continuum Photon Production Probability Density Function
$\mathrm{P}_{\mathrm{n}}$
Discrete Ordinance Order
$\phi_{n}(E)$
Energy Dependent Neutron Flux
$\phi_{\gamma}(E)$
Energy Dependent Photon Flux
$\varphi_{g}(\vec{r})$
Energy (Group-Wise) and Spatial Dependent Neutron Flux
$\ell$
Iteration Number
$\sigma_{\left(n, \gamma_{\_} \text {discrete }\right)}\left(E_{n} \rightarrow E_{\gamma}\right)$
Microscopic Cross Section for a Neutron with Energy $E_{n}$ to Produce a Discrete Gamma Ray with Energy $\mathrm{E}_{\gamma}$
$\sigma_{\left(n, \gamma_{-} \text {continuum }\right)}\left(E_{n} \rightarrow E_{\gamma}\right)$
Microscopic Cross Section for a Neutron with Energy $E_{n}$ to Produce a Continuum Gamma Ray with Energy $\mathrm{E}_{\gamma}$
$\sigma_{(n, \gamma)}^{i}\left(E_{g} \rightarrow E_{\gamma}\right)$
Microscopic Neutron Cross Section for a Reaction Producing a
Gamma Ray of Isotope i
$\sigma_{n}\left(E_{n}\right)$
Neutron Cross Section 

$\omega_{n}(E)$
Neutron Flux Approximation
$\mathrm{N}_{i}$
Number Density of Isotope $\mathrm{i}$
$\omega_{\gamma}(E)$
Photon Flux Approximation
$\sigma_{(n, \gamma)}\left(E_{n}\right)$
Photon Production Cross Section
$\mathrm{R}\left(E_{\gamma}, \vec{r}\right)$
Photon Production Rate
$y\left(E_{n} \rightarrow E_{\gamma}\right)$
Photon Production Yield
$\mathrm{p}\left(E_{n} \rightarrow E_{\gamma}\right)$
Probability that an Incident Neutron with Energy $\mathrm{E}_{\mathrm{n}}$ will produce a Continuum Gamma Ray with Energy $\mathrm{E}_{\gamma}$
$\mathrm{S}_{\mathrm{n}}$ Spherical Harmonics Scattering Order
$S(\alpha, \beta)$
Thermal Treatment of Bonded Atom
$\sigma_{t}^{i}(E)$
Total Microscopic Neutron Cross Section for Nuclide i 


\section{LIST OF ABBREVIATIONS}

CAD

DFEM

FSDS

GADRAS

GEB

HPGe

LCF

MCNP5

PGNAA

PINS

PWR

RADSAT

RADSAT-NG

ZAID
Computer Aided Design

Discontinuous Finite-Element

First-Scattered-Distributed Source

Gamma-Ray Detector Response Analysis Software

Gaussian Energy Broadening

High-Purity Germanium

Last-Collided Flux

Prompt Gamma Neutron Activation Analysis

Portable Isotopic Neutron Spectroscopy

Pressurized Water Reactor

RAdiation Detection Scenario Analysis Toolbox

RAdiation Detection Scenario Analysis Toolbox-Neutron Gamma

Isotope Identification Number 


\section{SUMMARY}

The accurate and efficient simulation of coupled neutron-photon problems is necessary for several important radiation detection applications. Examples include the detection of nuclear threats concealed in cargo containers and prompt gamma neutron activation analysis for nondestructive determination of elemental composition of unknown samples. In these applications, high-resolution gamma-ray spectrometers are used to preserve as much information as possible about the emitted photon flux, which consists of both continuum and characteristic gamma rays with discrete energies. Monte Carlo transport is the most commonly used modeling tool for this type of problem, but computational times for many problems can be prohibitive. This work explores the use of coupled Monte Carlo-deterministic methods for the simulation of neutron-induced photons for high-resolution gamma-ray spectroscopy applications.

RAdiation Detection Scenario Analysis Toolbox (RADSAT), a code which couples deterministic and Monte Carlo transport to perform radiation detection scenario analysis in three dimensions [1], was used as the building block for the methods derived in this work. RADSAT was capable of performing coupled deterministic-Monte Carlo simulations for gamma-only and neutron-only problems. The purpose of this work was to develop the methodology necessary to perform coupled neutron-photon calculations and add this capability to RADSAT. Performing coupled neutron-photon calculations requires four main steps: the deterministic neutron transport calculation, the neutroninduced photon spectrum calculation, the deterministic photon transport calculation, and 
the Monte Carlo detector response calculation. The necessary requirements for each of these steps were determined.

A major challenge in utilizing multigroup deterministic transport methods for neutron-photon problems was maintaining the discrete neutron-induced photon signatures throughout the simulation. Existing coupled neutron-photon cross-section libraries and the methods used to produce neutron-induced photons were unsuitable for high-resolution gamma-ray spectroscopy applications. Central to this work was the development of a method for generating multigroup neutron-photon cross-sections in a way that separates the discrete and continuum photon emissions so the neutron-induced photon signatures were preserved. The RADSAT-NG cross-section library was developed as a specialized multigroup neutron-photon cross-section set for the simulation of high-resolution gamma-ray spectroscopy applications.

The methodology and cross sections were tested using code-to-code comparison with MCNP5 [2] and NJOY [3]. A simple benchmark geometry was used for all cases compared with MCNP. The geometry consists of a cubical sample with a ${ }^{252} \mathrm{Cf}$ neutron source on one side and a HPGe gamma-ray spectrometer on the opposing side. Different materials were examined in the cubical sample: polyethylene $\left(\mathrm{C}_{2} \mathrm{H}_{4}\right), \mathrm{P}, \mathrm{N}, \mathrm{O}$, and Fe. The cross sections for each of the materials were compared to cross sections collapsed using NJOY. Comparisons of the volume-averaged neutron flux within the sample, volume-averaged photon flux within the detector, and high-purity gamma-ray spectrometer response (only for polyethylene) were completed using RADSAT and MCNP. 
The code-to-code comparisons show promising results for the coupled Monte Carlo-deterministic method. The RADSAT-NG cross-section production method showed good agreement with NJOY for all materials considered although some additional work is needed in the resonance region and in the first and last energy bin. Some cross section discrepancies existed in the lowest and highest energy bin, but the overall shape and magnitude of the two methods agreed. For the volume-averaged photon flux within the detector, typically the five most intense lines agree to within approximately $5 \%$ of the MCNP calculated flux for all of materials considered. The agreement in the code-to-code comparisons cases demonstrates a proof-of-concept of the method for use in RADSAT for coupled neutron-photon problems in high-resolution gamma-ray spectroscopy applications.

One of the primary motivators for using the coupled method over pure Monte Carlo method is the potential for significantly lower computational times. For the codeto-code comparison cases, the run times for RADSAT were approximately 25-500 times shorter than for MCNP, as shown in Table 1. This was assuming a $40 \mathrm{mCi}{ }^{252} \mathrm{Cf}$ neutron source and 600 seconds of "real-world" measurement time. The only variance reduction technique implemented in the MCNP calculation was forward biasing of the source toward the sample target. Improved MCNP runtimes could be achieved with the addition of more advanced variance reduction techniques. 
Table 1. Comparison of MCNP and RADSAT Computational Times for Simple Cube Problems

\begin{tabular}{|l|c|c|c|}
\hline Simulation & $\begin{array}{c}\text { RADSAT } \\
\text { (hours) }\end{array}$ & MCNP (hours) & Run-Time Ratio \\
\hline Polyethylene & 5 & 914 & 183 \\
\hline Phosphorus & 1.9 & 910 & 479 \\
\hline Nitrogen & 3 & 84 & 28 \\
\hline Oxygen & 3.5 & 93 & 27 \\
\hline Iron & 5 & 198 & 40 \\
\hline
\end{tabular}




\section{CHAPTER 1}

\section{BACKGROUND}

Neutron-induced photon signatures are an important component of many radiation detection scenarios. When a neutron interacts in matter, it can undergo a variety

of interactions that can produce gamma rays: radiative capture, inelastic scatter, $(n, 2 n)$, $(n, p),(n, \alpha),(n$, tritium), (n, deuterium), etc. These neutron-induced photon signatures can contribute significantly to the gamma-ray spectrum observed by the detector.

Many important radiation detection applications rely on the neutron-induced photon signatures as a primary focus of the gamma-ray spectrum. One such application includes the use of detectors at ports of entry used to identify nuclear threats concealed in cargo containers. Another application includes prompt gamma neutron activation analysis (PGNAA) for the nondestructive determination of the elemental composition of unknown samples. Both of these applications use high-resolution gamma-ray spectrometers to analyze the neutron-induced photon spectrum.

It is important to accurately predict the performance of these types of radiation detection systems. These predictions would not only aid in assessing the operational capabilities but could also identify promising paths in research. Ideally, these assessments are done through measurements in a realistic environment. Often, completing these measurements is either impractical or impossible due to time and cost constraints, or because the system does not yet exist. When performing measurements is not an option, numerical simulations become the tool of choice for calculating the detector response. 


\subsection{Computational Methods}

The methods for modeling radiation transport problems can be divided into two general categories: the stochastic method and the deterministic method. Additionally, a coupled stochastic-deterministic method can be used to simulate radiation transport problems. In the following sections, a detailed description of each method type and its associated advantages and disadvantages for use in simulating radiation detection scenarios will be discussed.

\subsubsection{Monte Carlo Methods}

The Monte Carlo method uses repeated random sampling of probability distributions to determine the solution to the problem. Monte Carlo simulations can be used to solve a variety of problems of interest in "nuclear reactor design, radiation shielding, nuclear criticality safety, decontamination and decommissioning, detector design and analysis, nuclear safeguards, accelerator target design, health physics, medical tomography and radiotherapy, nuclear oil well logging, waste storage and disposal, and radiography" [4] and are the preferred tool for simulating gamma-ray spectroscopy applications. A number of computer codes apply the Monte Carlo method for solving radiation transport problems including MCNPX [5], MCNP5 [2], Geant4 [6], Fluka [7], MARS [8], and Penelope [9]. The advantages and the disadvantages of the Monte Carlo method will now be discussed briefly.

The Monte Carlo method uses the sampling of probability distributions to simulate radiation transport. By generating a statistical analogue to describe the life of a particle, random numbers can be used to determine what interactions the particle will undergo along its flight path. In order for the analogue to properly represent the particle, 
the simulation must describe the physics of each interaction as accurately as possible [10]. In order to determine the results of the experiment, many particles must be simulated, and the expected value is obtained by determining the average behavior of the simulated particles and the statistical error associated with the average [10].

One important advantage of the Monte Carlo method is its ability to handle complex geometries without significant penalties in computational time. A Monte Carlo simulation offers the flexibility to complete calculations in one-, two- or threedimensions. Complex three-dimensional objects are represented by quadratic surfaces in the Cartesian coordinate system [10]. Unlike many deterministic computer codes, Monte Carlo simulations do not have problems handling curved geometries.

Another benefit of Monte Carlo computer codes is the use of point-wise cross section sets implemented for these calculations. (However, some Monte Carlo codes still use multigroup cross section sets.) Pointwise cross sections allow for the cross section at a specific energy be applied to the particle at that energy instead of an averaged cross section over an energy range. The cross section sets implemented by these codes have been well tested and closely approximate a continuous energy representation [1]. For example, MCNP uses cross sections in an ACER format on an energy grid which allows for linear interpolation between points; this interpolation scheme allows for a specified tolerance within the continuous energy cross section [2].

The other major benefit of using a Monte Carlo simulation is the statistical nature of the calculation. Simulating a given number of particles in a Monte Carlo code produces a solution with a statistical uncertainty. When determining what a detector could or could not see during a given count time, detector analysts will model the number 
of particles produced during that period instead of trying to achieve a desired level of statistics thus introducing statistical noise into the system. This allows the analyst to determine if the region of interest is observable given the permitted count time. By modeling the detector response using the Monte Carlo method, the statistical nature of the problem is maintained.

Additionally, there are a number of shortcomings to overcome when utilizing Monte Carlo computer codes. Certain classes of problems can be time consuming when using the Monte Carlo method. When dealing with problems with a high degree of scatter or attenuation using analog Monte Carlo, tracking each interaction for each individual particle can become computationally expensive and cumbersome since most of the computational time is spent tracking particles that do not significantly contribute to the desired result. In order to obtain a solution with an acceptable level of error, two approaches can be implemented: run more particles or apply variance reduction techniques. Sometimes running more particles is not an option, since increasing the number of particles simulated increases the computation time required to achieve a statistically acceptable result. Additionally, the number of particles able to be simulated is dictated by the size of the random number generator. If the number of simulated particles exceeds the maximum sample size dictated by the random number generator, the simulation may yield results of sufficient precision but not accuracy (i.e. it may contain correlated samples) [10].

Alternatively, variance reduction techniques can be implemented in Monte Carlo simulations in order to reduce the number of particles sampled to achieve the desired statistical accuracy in the result, thus reducing the computational time. Implementing 
variance reduction techniques requires additional time to setup the problem and is only valid for the problem at hand. Additionally, there are some types of problems where variance reduction methods provide little or no benefit.

Another major obstacle is that the Monte Carlo calculations do not provide a global solution, only the specific solution requested in the input file. If the detector location needs to be altered in a Monte Carlo simulation, the solution at the new location will have to be calculated using an entirely new simulation.

For high-resolution gamma-ray spectroscopy applications, the Monte Carlo method is the preferred method for numerical simulations by detector analysts. Monte Carlo codes allow for direct simulation of the pulse-height tally in the detector. Although Monte Carlo methods provide an accurate solution and a direct calculation of the pulseheight tally, the method can be computationally expensive when dealing with problems where scattering and attenuation are prevalent.

\subsubsection{Deterministic Methods}

Deterministic methods are commonly applied to nuclear reactor analysis and shielding problems; the method can also be applied to problems dealing with radiological safety, medical imaging, radiotherapy, fuel transport and storage, fusion research, homeland security and oil exploration [11]. Typically deterministic methods are not used to simulate high-resolution gamma-ray detection scenarios.

Deterministic transport codes, such as THREEDANT [12], PARTISN [13], TORT [14] and Attila [6], implement an approximation to the linearized Boltzmann transport equation; the most common of which is the discrete-ordinates method. Deterministic transport requires the discretization of the integro-differential transport 
equation over space, energy and time. The discrete-ordinates method is obtained by additionally discretizing the angular variable, creating a small number of discrete directions or rays. A global solution is obtained by numerically solving the set of algebraic equations derived through the discretization process [10].

One of the main advantages of using a deterministic code is that it produces a global flux solution. Unlike in a Monte Carlo code where the user is required to define the output prior to the calculation, deterministic codes allow the user the ability to determine the solution to the calculation at any location in the problem regardless of whether or not it was specified prior to the calculation. This feature can be extremely advantageous in a detection scenario if the analyst is interested in moving the location of the detector or determining the proper location to place a detector. If the detection system is small enough that it does not directly affect the flux solution within the problem, the ability to obtain a global flux solution offers some flexibility to the problem. For this class of problems, the flux solution at any point in the problem can be obtained from the global flux solution without having to re-solve the transport problem.

Another advantage of using a deterministic code is that it produces an "infinite time solution," or a solution which does not contain the statistical uncertainty associated with Monte Carlo calculations. This is extremely advantageous when simulating longdwell detection scenarios where little statistical noise is present and Monte Carlo simulations are very time consuming. Because deterministic transport codes calculate a numerical solution to the discretized transport equation, the code computes a solution without the statistical uncertainty associated with Monte Carlo simulations. 
The third major advantage of using a deterministic code is their ability to achieve shorter computational times compared to Monte Carlo for certain classes of problems. Unlike in Monte Carlo calculations where computational time requirements is proportional to the number of particles simulated, the computational requirements for deterministic codes are directly affected by the number of discrete bins in space, energy and angle. For problems dominated by scattering and attenuation, discrete-ordinates codes have the potential to produce accurate results in relatively short computational times, when compared to Monte Carlo. This assumes that a reasonable geometric mesh size can be utilized in the problem [1].

In order to obtain accurate results using the discrete-ordinates approximation, it is important to understand the possible causes of errors. Unlike Monte Carlo simulations which implement close approximations to continuous energy cross sections, the deterministic method requires discretization in energy, resulting in multigroup cross sections. The ability to properly preserve the physics of the problem is directly related to the fidelity of the cross sections. If the cross section is varying rapidly within the energy group, errors can result from the approximation of the cross section for the group. Additionally, the methods used for the discretization of the geometry may result in some limitations on the types of geometric shapes that can be properly approximated. For instance, if a Cartesian coordinate mesh was used (i.e. cubes with faces parallel to the x-, $y$ - and z-plane), the approximation may result in errors for volumes of spheres or other curved surfaces.

The main disadvantage to employing a discrete-ordinates code to a detector response problem is the ray effect. In the discrete-ordinates method, particles are 
assumed to travel along discrete directions while preserving the overall flux. The amount and spacing of these discrete directions is determined by the order of the discreteordinates calculation. Low order calculations of uniform sources artificially bias the particles to travel in only a few discrete directions. This biasing of particle travel causes artificial enhancement and depression of the particle flux known as ray effects [15]. An example of the ray effects calculated of the scalar flux at a constant distance from a point source in air using a discrete ordinates approximation is shown in Figure 1 [1]. The discrete ordinates calculation should produce a uniform flux over all angels at a given distance from the source in air. At the lower discrete ordinates, the flux is artificially increased at some angles and decreased at others. As the $S_{n}$ order is increased, the flux approaches a uniform solution but is computationally expensive. When modeling the detector location, it is possible to choose a position where the flux is artificially high or low. In order to mitigate this effect, the detector simulation should be carried out using a high-order angular approximation and/or using a first-collision code to generate a first collision source which will lead to an increase in the computational time required to converge on a global solution [1]. 


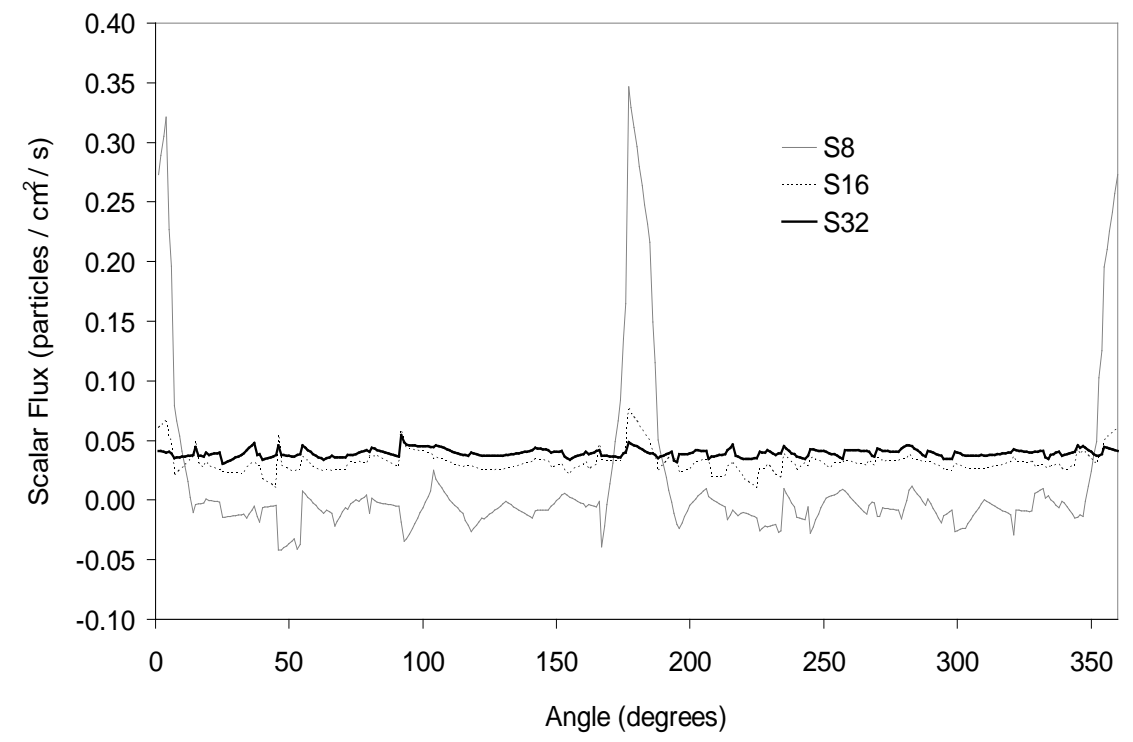

Figure 1: Example of Ray Effects Resulting from the Calculation of the Scalar Flux for a Point Source in Air. $S_{8}, S_{16}$, and $S_{32}$ represent the $S_{n}$ order used in the discrete ordinates calculation.

The other major issue encountered when using a deterministic code to simulate high-resolution gamma-ray detector response is the nature of the method. Detector response is a stochastic process. Although some work is being done to simulate the detector response directly using deterministic transport, the detector response is not typically directly simulated using deterministic transport approximations. The effects of many of the discretization errors described and the statistical nature of the detector response can be mitigated by coupling the discrete-ordinates code to a Monte Carlo simulation to obtain the detector response.

\subsection{Coupled Stochastic-Deterministic Methods}

Coupled stochastic-deterministic methods offer some direct advantages over pure stochastic or deterministic simulations for certain problems with a high degree of scattering or attenuation. It is possible to exploit the positive aspects while mitigating 
some of the negative aspects of each of the methods acting individually. Since discreteordinates calculations offer advantages for determining the global flux solution, the general transport calculation is solved using a discrete-ordinates code. Due to the statistical nature of the stochastic method and its ability to accurately represent a pulseheight tally in a gamma-ray spectrometer, the final transport through the detector is simulated using a Monte Carlo code.

The coupled stochastic-deterministic method offers a number of advantages for high-resolution gamma-ray detector applications. By employing a discrete-ordinates code to calculate the global field solution, it allows for the physical size of the Monte Carlo problem to be significantly reduced, which reduces the computational time required to achieve a solution for some cases. This coupled approach also applies some additional flexibility to the simulation. For instance, if the location of the detector needs to be altered from its original location in the simulation, the flux at the new location can be extracted from the global flux solution provided from the discrete-ordinates calculation. The only additional computational time required to determine the detector response is the Monte Carlo simulation directly around the detector at the new location. The reduction in computational time requirements and the added flexibility makes the coupled discrete ordinates and Monte Carlo method attractive for certain classes of problems.

Radiation Detection Scenario Analysis Toolbox (RADSAT) is a computer interface which aids in the coupling of discrete-ordinates and Monte Carlo calculations for use in determining detector response. Attila, a discrete-ordinates code which was originally developed at Los Alamos National Laboratory and is currently updated and maintained by Transpire Inc. [16], is used to calculate the global flux solution, and Monte 
Carlo N-Particle Version 5 (MCNP5) [2], which was also developed at Los Alamos National Laboratory, is used to calculate the detector response. For some instances Gamma-Ray Detector Response Analysis Software (GADRAS), a one-dimensional deterministic transport code developed at Sandia National Laboratory [17], is used in place of MCNP to calculate the detector response. Each of these computer codes were chosen for a combination of reasons: computational abilities, features, and user familiarity.

The implementation of Attila as the deterministic core of RADSAT was chosen over other deterministic codes due to the inclusion of several attractive features. Unlike most deterministic codes which employ the Cartesian coordinate system for meshing, Attila solves the transport equation using an unstructured tetrahedral mesh, allowing Attila to provide more accurate approximations to complicated geometries such as curved surfaces. Attila also has the ability to incorporate ray-tracing which is well suited for radiation detection problems and aids in the mitigation of ray effects. In order to minimize the ray effects in point source simulations, Attila implements a method called the "first-scattered-distributed source" (FSDS). The FSDS calculation consists of completing the "first-scattered" calculation to obtain a distributed source that is then used to determine the scattered source. Ray tracing is then used to determine the uncollided flux. The sum of these two components is used to determine an accurate global flux profile for the problem [1]. The second tool used to mitigate ray effects in Attila is the "last-collided flux" method or LCF. Once the global flux solution is obtained, the integral transport is applied to the user-defined edit point by transversing the mesh along many individual lines of sight to account analytically for absorption and scattering. An 
LCF calculation is depicted in Figure 2. Without the ability to mitigate ray effects, the computation of the flux on a small detector would result in erroneous solutions.

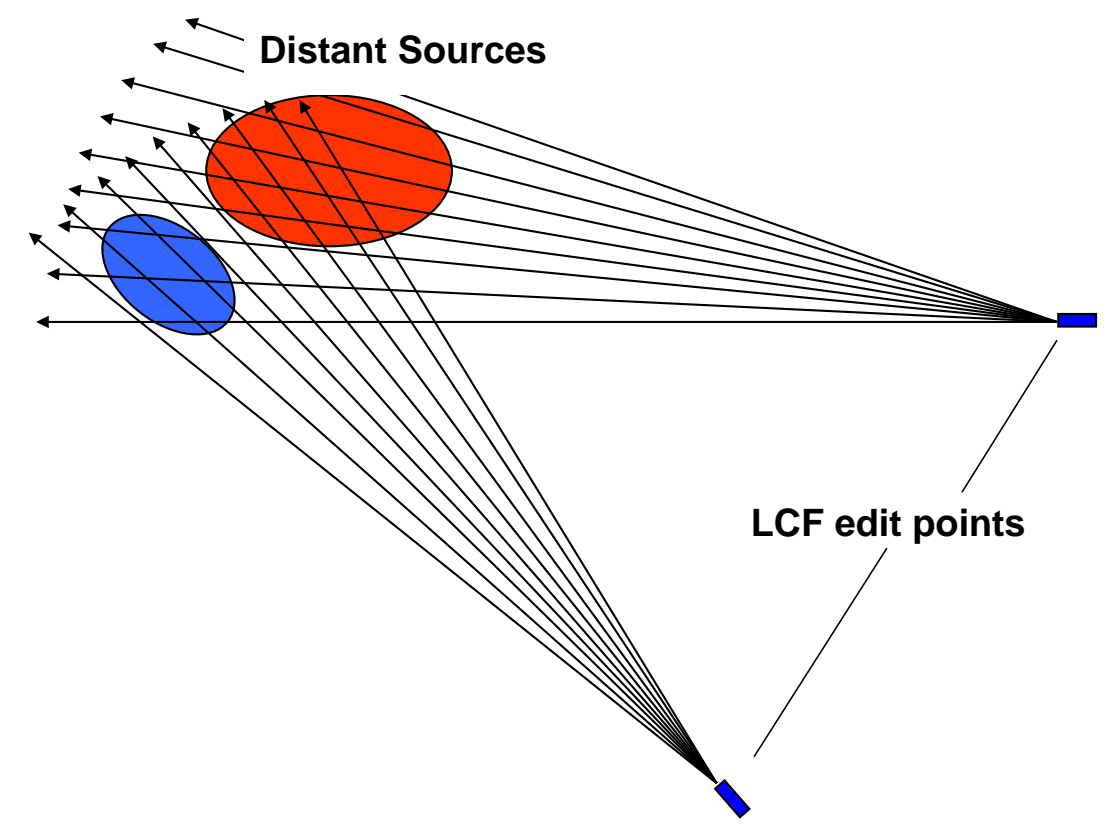

Figure 2: Illustration of last-collided flux (LCF) edit as a means to mitigate ray effects in discrete ordinates solutions [1]

Employing MCNP instead of other Monte Carlo codes such as Geant4 offers some advantages. The Geant 4 input file is written in $\mathrm{C}$ and requires the user to identify the specific physics packages required to solve the problem [6]. Alternatively, the MCNP5 input file is more of a fixed format containing default physics packages. The user is only required to specify the geometry and materials of the detector itself. RADSAT generates the remainder of the input file for MCNP5 using the output file from Attila and the source subroutine module added to MCNP5. Although development up to this point has focused on applying MCNP5 as the Monte Carlo backend for RADSAT, any Monte Carlo simulation code could be used to complete these calculations. 
One alternative to calculating the detector response using MCNP is to employ a deterministic code to complete the calculation. The radiation detection community is familiar with the potential advantages of deterministic transport, particularly in terms of computational time for problems that can be reduced to one-dimensional approximations. This reduction to one-dimension eliminates the problems due to ray effects completely. GADRAS uses these approximations when calculating the transport in its base code ONEDANT. Once the one-dimensional transport equation has been solved, GADRAS utilizes a semi-empirical "calibration" approximation to calculate the detector response, where the one dimensional flux is assumed to be incident on a specific face of the sensor [17]. Although these methods are extremely fast at producing results, even for deep penetration problems, they "are not adequate for more complex detection scenarios, in which one-dimensional approximations are not sufficiently accurate or when using detector types that preserve directional information" [1].

Currently RADSAT has the capability to simulate detector response for neutrononly or photon-only scenarios. It cannot complete a high resolution gamma-ray calculation for photons produced by neutron interactions within the simulation. Attila possesses the capability to generate a photon spectrum from neutron interactions in coarse energy bins within the local environment, as long as the cross-section library provided contains the necessary information. For instance, the Radion 15 cross-section library contains cross section data for twenty-two neutron groups (Groups 1-22) and twenty-five photon groups (Groups 23-47) [18]. When a neutron interacts producing a gamma-ray, it appears as a between group scatter starting in a neutron group and scattering into a photon group. For example, a 2-MeV neutron (Group 10) interacts with 
a hydrogen atom producing a 2.223-MeV gamma-ray (Group 35). This interaction would look like a neutron scattering from Group 10 to Group 35, and no distinction between particle types would be made. This coarse energy group photon spectrum will not preserve the discreteness of photopeaks and therefore will not contain the level of detail required for use in high-resolution gamma-ray spectroscopy applications. In order to obtain the required level of detail for this application in RADSAT, a separate module was constructed to calculate the detailed photon continuum and photopeaks separately in a form that Attila can use to simulate the photon transport.

\subsection{Applications of Coupled Method}

The accurate and efficient simulation of coupled neutron-photon problems is necessary for several important radiation detection applications. Adding the capability to simulate coupled neutron-photon problems using RADSAT has many applications including the simulation of nondestructive assay of a uranium-hexafluoride $\left(\mathrm{UF}_{6}\right)$ cylinder, radiation portal monitor, handheld instruments used to survey people and packages, and other nondestructive assay (NDA) scenarios. The exclusion of the neutron-induced photon spectrum could omit a significant feature of the spectrum for each of these examples. The following sections give more detailed explanations of the applications and benefits of using RADSAT for coupled neutron-photon problems.

\subsubsection{Interrogation of Cargo Containers}

During the screening of vehicles and cargo at a border crossing, a neutron source within a cargo container may induce gamma rays in the surrounding cargo as shown in Figure 3. To simulate this scenario it is necessary to simulate the neutron-induced 
photons or the detectors will not register a source within the vehicle. RADSAT would currently only have the capability to simulate counts in the gamma-ray detectors from gamma-ray sources explicitly defined within the problem.

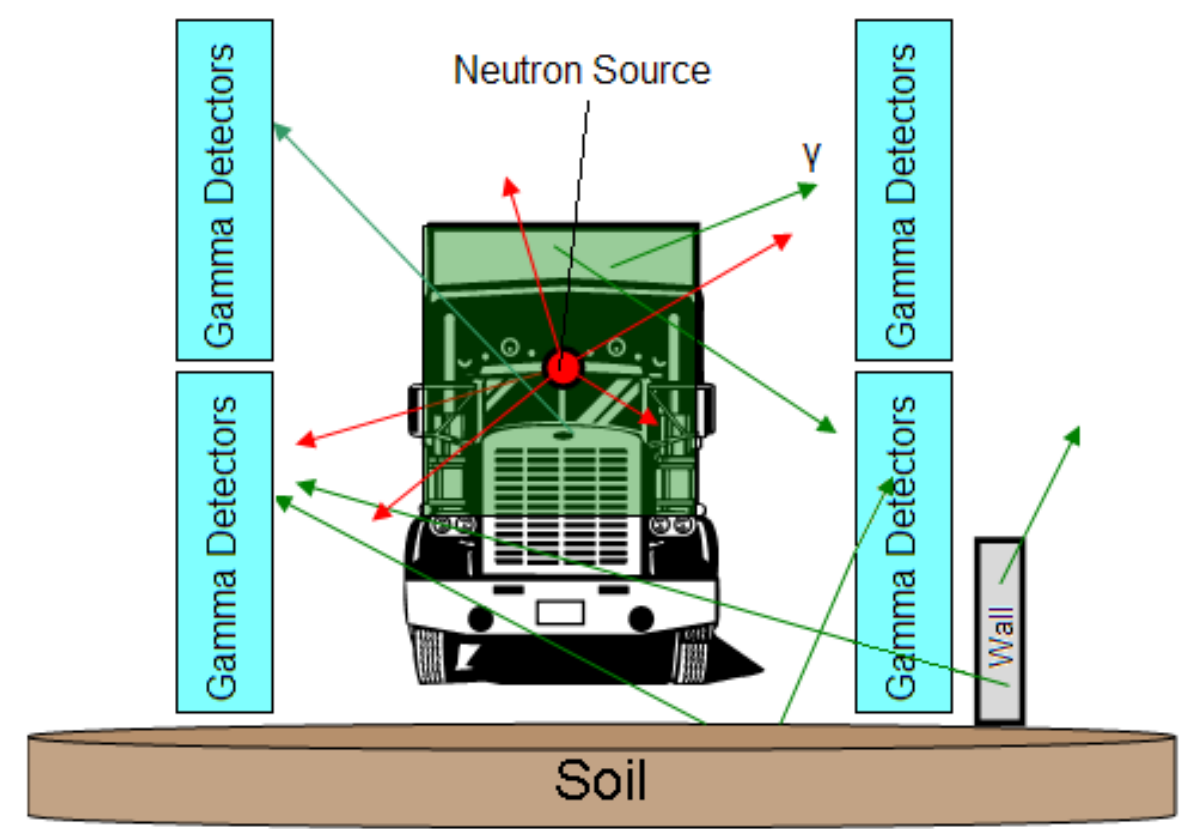

Figure 3. Passive Interrogation of Cargo Container Using Gamma Detectors to Find Gamma Rays Produced by Neutron Interactions in the Container

The RADSAT coupled method offers many advantages for the simulation of passive interrogation scenarios. The interrogation of cargo containers is very complex. These are three dimensional problems, usually containing multiple sources and including deep penetration. The large physical scale of the problem relative to the detector size makes applying pure Monte Carlo simulations extraordinarily time consuming [19]. Also, many possible scenarios must be considered for this class of problems to determine the response of the detectors to different classes of cargo so minimizing the computation 
time is desired. With the addition of the coupled neutron photons, RADSAT is a promising tool for solving passive interrogation problems.

\subsection{2 $\mathrm{UF}_{6}$}

The verification of declared enrichment in $\mathrm{UF}_{6}$ cylinders produced at enrichment facilities is a big component of the international safeguards regimes. Various NDA methods are being utilized and considered for this application. Signatures to examine include both neutrons and gamma-rays. A significant portion of the gamma-ray spectrum produced from the $\mathrm{UF}_{6}$ is due to neutron-induced photons. A diagram of a $\mathrm{UF}_{6}$ cylinder and the general arrangement of such a facility are shown in Figure 4.

Being able to properly predict the results of placing a detection system around a $\mathrm{UF}_{6}$ cylinder in a configuration similar to the one depicted in Figure 4 using RADSAT for the purpose of inventory would be extremely advantageous. The $\mathrm{UF}_{6}$ cylinders contain a high degree of self-attenuation. Additionally, the cylinders are not isolated from each other, resulting in a high radiation background environment. The combination of these two factors makes simulating this scenario in MCNP challenging. In order to properly simulate the gamma-ray spectrum, the neutron-induced photon spectrum must be taken into account. 


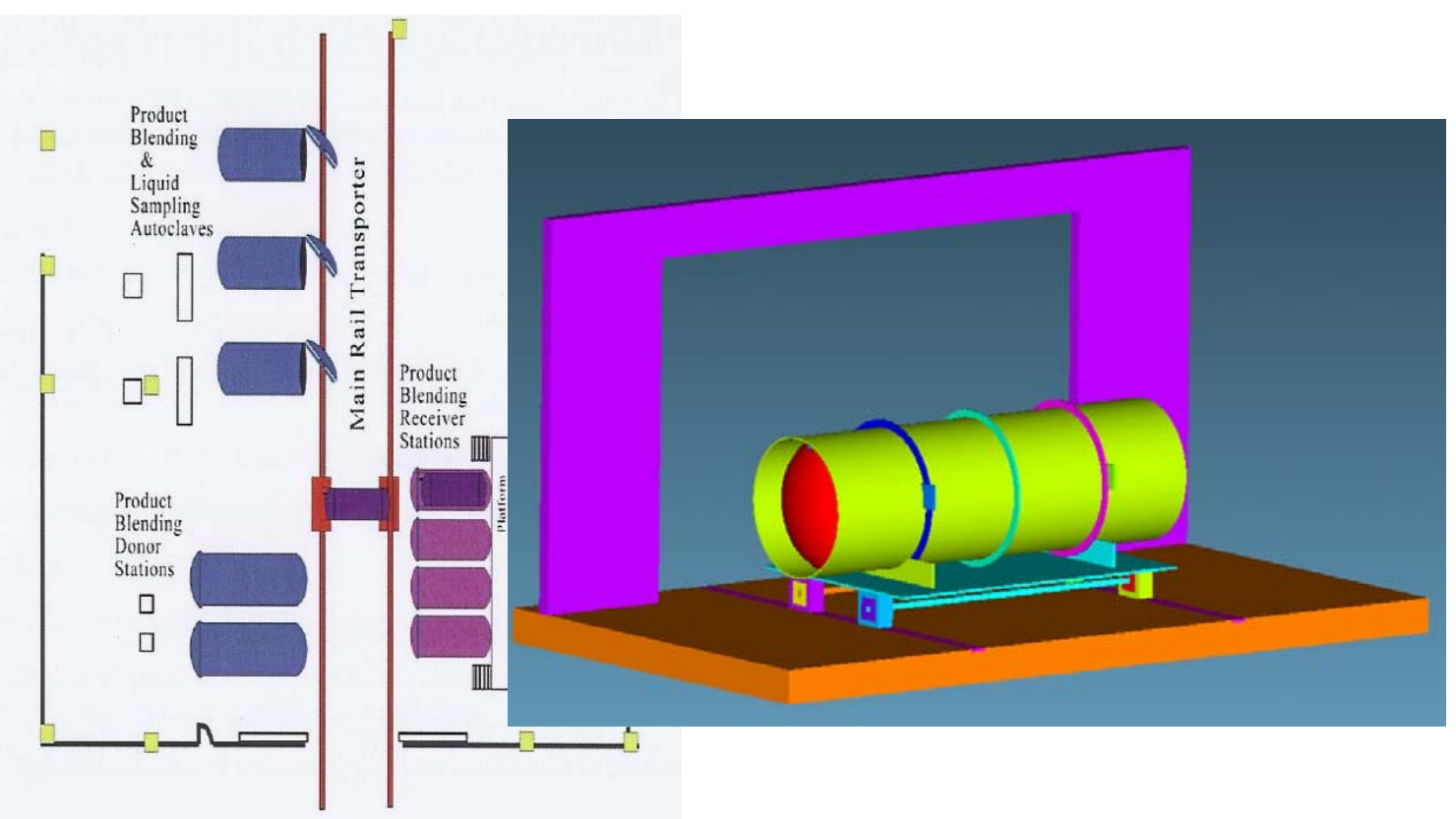

Figure 4: $\mathrm{UF}_{6}$ Cylinder and Typical Facility Layout

\subsubsection{Prompt Gamma Neutron Activation Analysis}

PGNAA is a form of active interrogation in which neutrons are used to actively interrogate a package and nondestructively determine its contents. In this analysis, the material is irradiated with a neutron source. The neutrons interact in the material and produce gamma rays through a number of interactions, including inelastic scattering and radiative capture. An isotope can be identified using the discrete gamma rays produced from the neutron interactions. For example, hydrogen produces a $2.223-\mathrm{MeV}$ gamma ray as a result of radiative capture. The presence and relative portions of these discrete gamma rays can be used to infer the chemical composition and relative abundance of the constituents within the material. In order to obtain the level of detail required to identify particular gamma-rays, the use of a high-resolution gamma-ray spectroscopy system is 
required. Without the ability to account for neutron-induced photons, RADSAT will be unable to simulate neutron activation problems altogether.

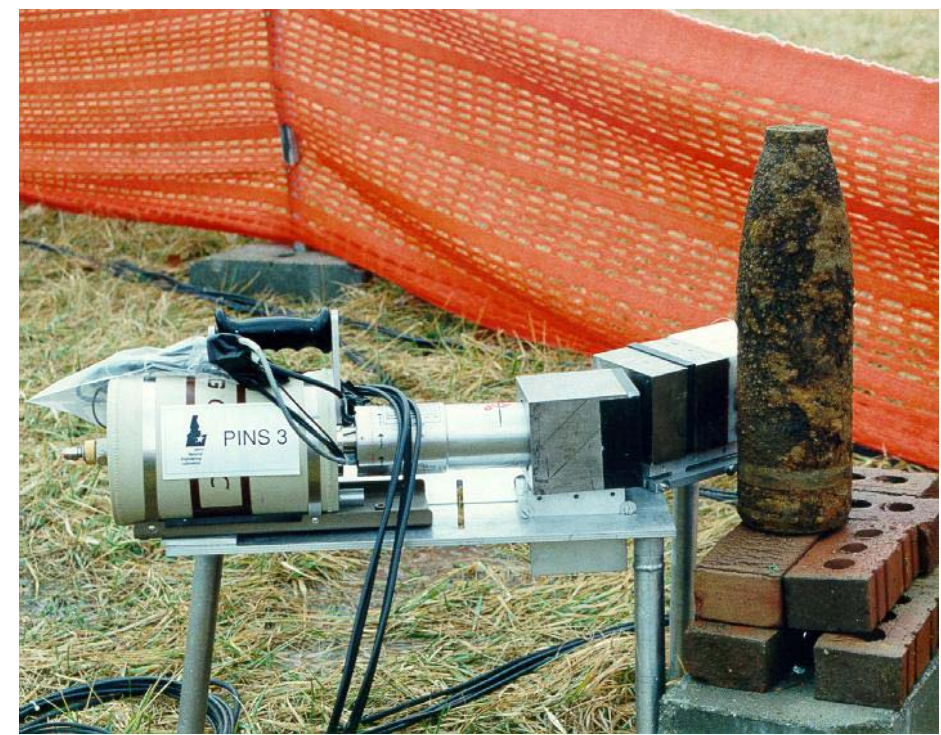

Figure 5. Prompt Gamma Neutron Activation Analysis Setup 


\section{CHAPTER 2}

\section{METHODS}

Can a coupled Monte Carlo-deterministic transport code be used for the simulation of high-resolution neutron-induced gamma-ray spectroscopy? The desired outcome of this research was to add the capability to produce the detector response from a neutron-induced photon spectrum using a coupled deterministic stochastic approach to RADSAT.

\subsection{Experimental Design for Testing Methods}

During the initial design phase for the RADSAT coupled neutron-gamma methods, completing some of the code-to-code comparison in parallel with the development of the methodology was necessary. As a basis for testing the neutrongamma methodology proposed for RADSAT, the RADSAT results were compared to MCNP5 results for a series of simple neutron-activation problems. The geometric configuration for these problems is shown in Figure 6 where a ${ }^{252} \mathrm{Cf}$ neutron source was placed $5 \mathrm{~cm}$ from one face of an $8000 \mathrm{~cm}^{3}$ cube composed of various homogenous materials. A coaxial high-purity germanium (HPGe) spectrometer was placed $5 \mathrm{~cm}$ away from the opposing face of the cube. 


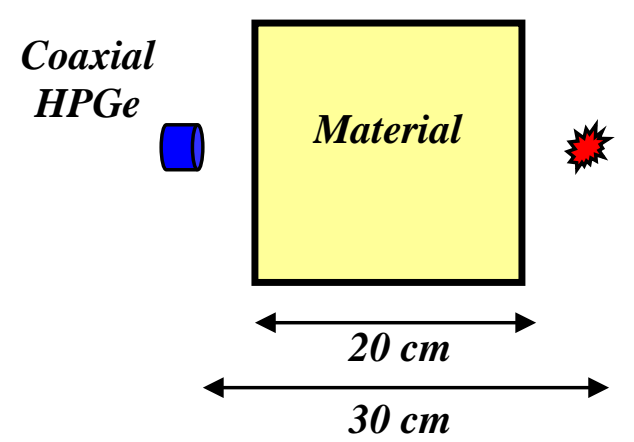

Figure 6. Source-sample-detector geometry for the neutron activation analysis problems used in code-to-code comparisons of RADSAT and MCNP5.

The simulated Ortec Detective HPGe has an active crystal dimension of approximately $5 \mathrm{~cm}$ in diameter by $3 \mathrm{~cm}$ thick with a relative efficiency of approximately $10 \%$. The full-width at half-maximum energy resolution is approximately $3-\mathrm{keV}$ at 1333-keV. A detailed graphic of the detector is shown in Figure 7. The magenta is the germanium crystal in the detector, the white is modeled as a vacuum within the detector volume, the dark blue is the aluminum detector casing, the light blue is the plastic cap on the front of the sensor, the yellow is the thin inner can cap which is modeled as low density carbon $\left(1.01 \mathrm{grams} / \mathrm{cm}^{3}\right)$, and the green is the air surrounding the detector. This detector model had previously been utilized for another RADSAT application and was validated [1]. The original model contained a large region $(10 \mathrm{~cm} \times 10 \mathrm{~cm} \times 7.5 \mathrm{~cm})$ of homogenized polyethylene and aluminum $\left(14.25 \mathrm{grams} / \mathrm{cm}^{3}\right)$ behind the detector to simulate the batteries and electronics. Although this region would provide backscatter into the detector and perturb the neutron flux within the cube of moderating material, it was removed in order to examine only the induced-photon spectrum produced by the neutron irradiation of the target sample. 


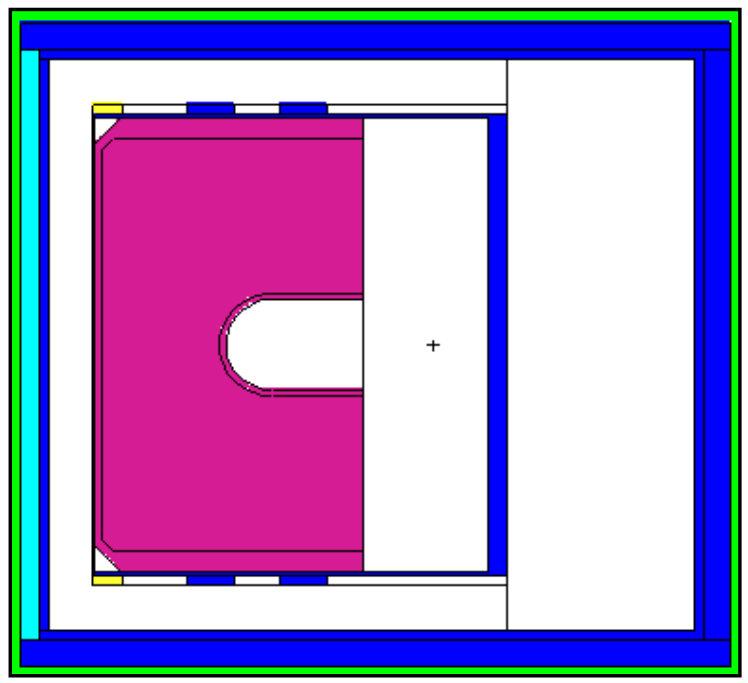

Figure 7.Schematic of the Ortec Detective HPGe. (See text for a color-coded description of the detector region)

During the method development, the composition of the target material cube was first simulated as low density $\left(0.5 \mathrm{~g} / \mathrm{cm}^{3}\right)$ polyethylene $\left(\mathrm{C}_{2} \mathrm{H}_{4}\right)$. Tests were conducted using the polyethylene case to determine the effects of the neutron cross section used to solve the deterministic neutron transport problem and the spatial dependence of the neutron-induced photon spectrum. The results of these tests are discussed in the Methods Discussion Section. The same geometric configuration was also used to test several other material compositions including nitrogen, oxygen, phosphorus, and iron as discussed in the RESULTS Chapter.

\subsection{Methods Discussion}

Applying a coupled deterministic-Monte Carlo methods to a coupled neutronphoton problem requires the simulation to be partitioned into multiple sections.

Obtaining a high-resolution detector response for coupled neutron-gamma simulations requires four steps: 
1) A multigroup, deterministic neutron transport calculation of the source neutrons to all parts of the problem;

2) The generation of the corresponding spatially dependent neutron-induced gammaray spectrum;

3) A multigroup, deterministic photon transport calculation of the induced gammaray spectrum to all parts of the problem;

4) The calculation of the detector response (i.e. pulse-height tally) in a spectrometer with a Monte Carlo code.

The process is outlined in Figure 8. The methods and motivation for solving the neutron transport, photon transport to the detector location, and the detector response are previously included in RADSAT, but understanding how to utilize the individual features to solve the coupled neutron-photon simulation had not previously been addressed. The focus of the research was the development of a neutron-induced photon calculation. An explanation for completing each of the steps in Figure 8 follows. 


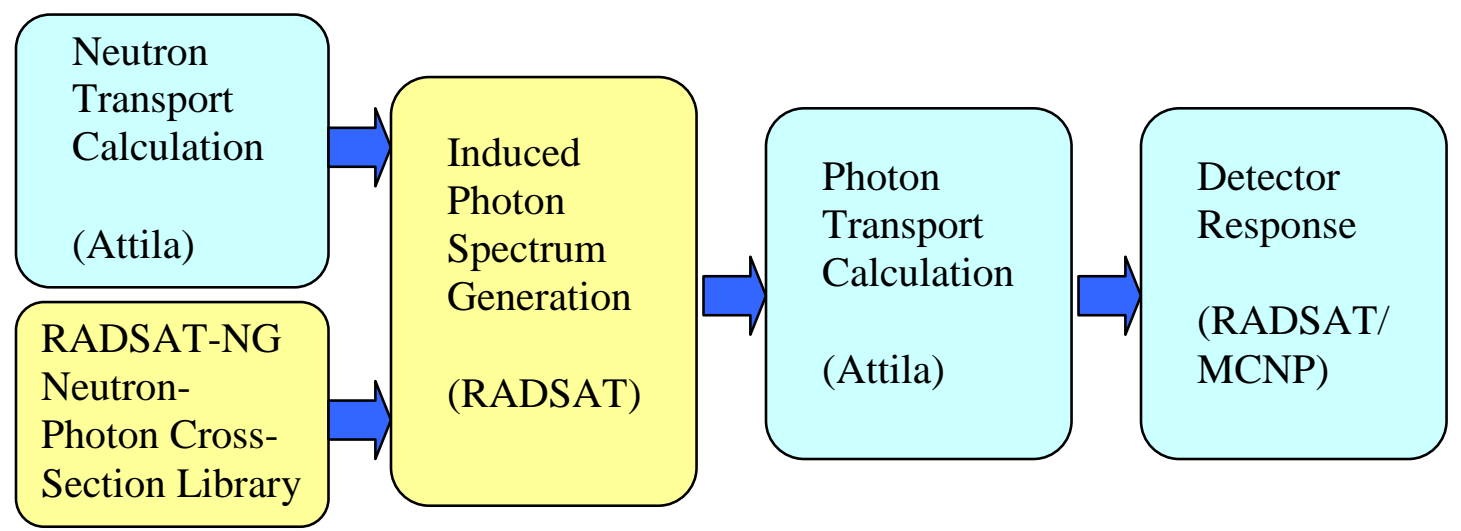

Figure 8. Procedure for Calculating Coupled Neutron-Photon Problems Using RADSAT. The blue boxes (neutron transport, photon transport and detector response) apply principles previously included in RADSAT. Yellow boxes (RADSAT-NG cross-section preparation and neutron-induced photon source generation using RADSAT) are unique steps developed in this work for coupled neutron-photon problems.

\subsubsection{Neutron Transport Calculation}

The first step in performing a coupled neutron-photon simulation is the neutron transport calculation using Attila. Therefore, the neutron transport utilizes a standard discrete-ordinates calculation, which is not unique to RADSAT. The required output of the neutron transport calculation is a volume-dependent neutron flux with which to calculate the neutron-induced photon spectrum. An example Attila neutron transport calculation input file for polyethylene is shown in APPENDIX A. The following sections explain the choice of neutron cross sections library, spatial dependence, transport parameters, reports, and the parameters used to determine solution convergence.

\subsubsection{Neutron Cross-Section Libraries}

The accuracy of the neutron-induced photon spectrum is directly related to the accuracy of the neutron flux which is dependent, but not solely so, on the neutron cross sections used to solve the problem. The multigroup cross sections are weighted averages 
of the cross section over energy bins. The more neutron energy groups in the multigroup neutron cross-section library, the closer the representation is expected to be to the continuous energy cross section. Conversely, an increase in the number of neutron groups used to calculate the energy-dependent neutron flux in a discrete-ordinates code is directly related to the time required to obtain the global flux solution. It is desirable to achieve high degree of detail in the neutron spectrum while maintaining low computational requirements. Due to the high correlation between the energy of the neutron and both the neutron cross section and the energy of the resulting photon, it is important that the neutron interaction cross sections and the neutron flux are sufficiently resolved to allow for the proper computation of the resulting photon spectrum.

There exist a variety of multigroup neutron cross-section libraries designed to obtain solutions to an acceptable degree of accuracy for specific classes of problems. The physics of certain problems may require fine energy grouping structure in certain regions and allow coarser energy groups in other regions. There are many different reactions which may produce a gamma ray spanning a wide range of energies such as radiative capture, inelastic scatter, $(n, 2 n),(n, p),(n, \alpha),(n$, tritium), (n, deuterium), etc., so the energy group structure of the multigroup cross section can have a dramatic effect on the fidelity of the neutron-induced photon spectrum calculation. Some of these interactions are driven primarily by thermal neutron interactions (i.e. radiative capture in carbon has a thermal cross section of 0.135 barns and a total cross section of 0.137 barns). Conversely, threshold interactions which produce gamma rays (i.e. inelastic scatter in carbon) are dependent on the interactions of high-energy neutrons in the material. Three cross-section libraries were examined to assess which ones provide an 
adequate multigroup approximation to the neutron flux for the generation of neutroninduced photon spectra: Radion15 [18], SCALE44 [20], and Kynea3 [21].

The Radion 15 cross-section library was originally developed for use with Attila to solve shielding problems. The Radion 15 cross-section library consists of twenty-two neutron energy groups and twenty-five photon groups. It employs a coarse neutron group structure in the thermal energy range, containing only three energy groups below $3.5-\mathrm{eV}$, and provides more detail in the high-energy region, containing 10 energy groups above $2-$ $\mathrm{MeV}$.

The SCALE44 group cross-section library was the second cross-section library considered for use in solving the neutron transport calculation for coupled neutronphoton problems. The SCALE44 group library was developed by collapsing the SCALE 238 group library using a PWR spectrum. It was developed for the "analysis of fresh and spent fuel and radioactive waste systems" [20]. Since nuclear reactor analysis is highly dependent on the thermal neutron flux, the SCALE 44 group cross-section library consists of twenty-two energy groups below $3-\mathrm{eV}$ with upscatter and only one energy group above $8-\mathrm{MeV}$.

The Kynea3 cross-section library was the final cross-section library to be considered for use in neutron transport calculations for coupled neutron-photon problems. Unlike the Radion15 and SCALE44 cross-section libraries previously described, Kynea3 was developed as a pseudo-problem independent cross-section library. Kynea3 was obtained by combining the 35 thermal energy groups with upscatter from the VITAMINB6 cross-section library [22] with the 44 higher energy groups from the SNAPPLE crosssection library [23]. Each of the neutron energy group structures previously mentioned 
offers a different representation of the cross-section based on the intended use of the library. A comparison of the neutron energy group structure is shown in APPENDIX B. As a semi-quantitative determination of the most appropriate cross-section library to use for induced photon calculation, a direct comparison of the photon production rates for hydrogen, carbon and deuterium was calculated. The volume-averaged neutron flux within the entire polyethylene cube was calculated in Attila using each of the crosssection libraries: Radion15, SCALE44 and Kynea3. The neutron flux results obtained from each Attila calculation are compared to an MCNP calculation in Figure 9 and Figure 10. The MCNP, SCALE44 and Kynea3 simulations all use $S(\alpha, \beta)$ treatments. The total neutron fluxes calculated in Attila using SCALE44 and Kynea3 were within $1 \%$ of the MCNP simulation. The $S(\alpha, \beta)$ treatment takes into account the thermal scattering of the bonded hydrogen atom. Without the $S(\alpha, \beta)$ treatment, the thermal scattering in hydrogen is treated as a free gas. The Radion 15 cross-section library does not contain the $S(\alpha, \beta)$ treatment for polyethylene which accounts for the $-90.4 \%$ and $539 \%$ difference from the MCNP calculation in neutron energy groups 21 and 22, respectively. The total neutron flux calculated in Attila using Radion15 was within 14.3\% of the MCNP simulation. The MCNP input file is show in APPENDIX C. 


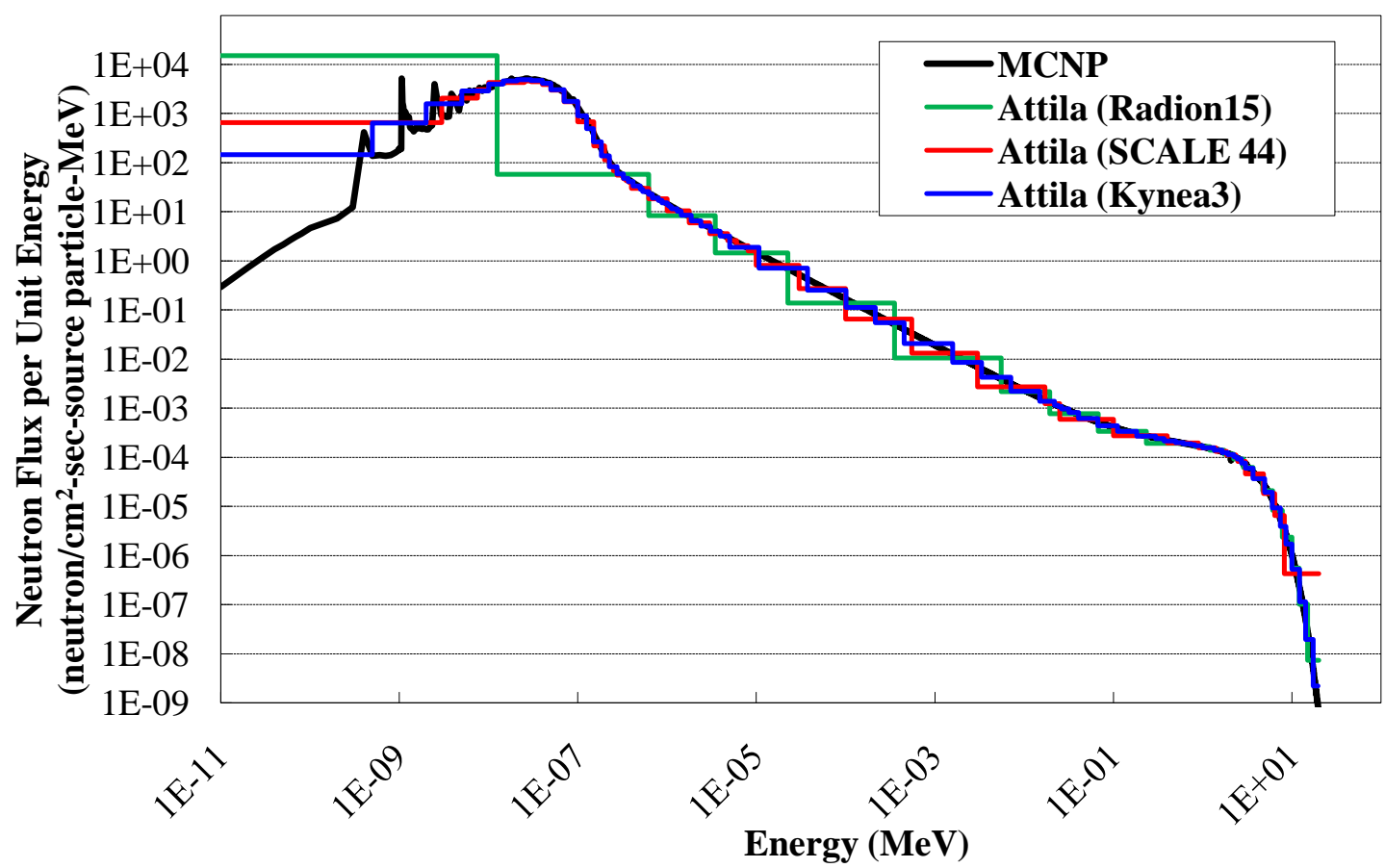

Figure 9. Comparison of Volume-Averaged Neutron Flux in Polyethylene Cube from MCNP and Attila using Radion15, SCALE44 and Kynea3 Cross-Section Libraries

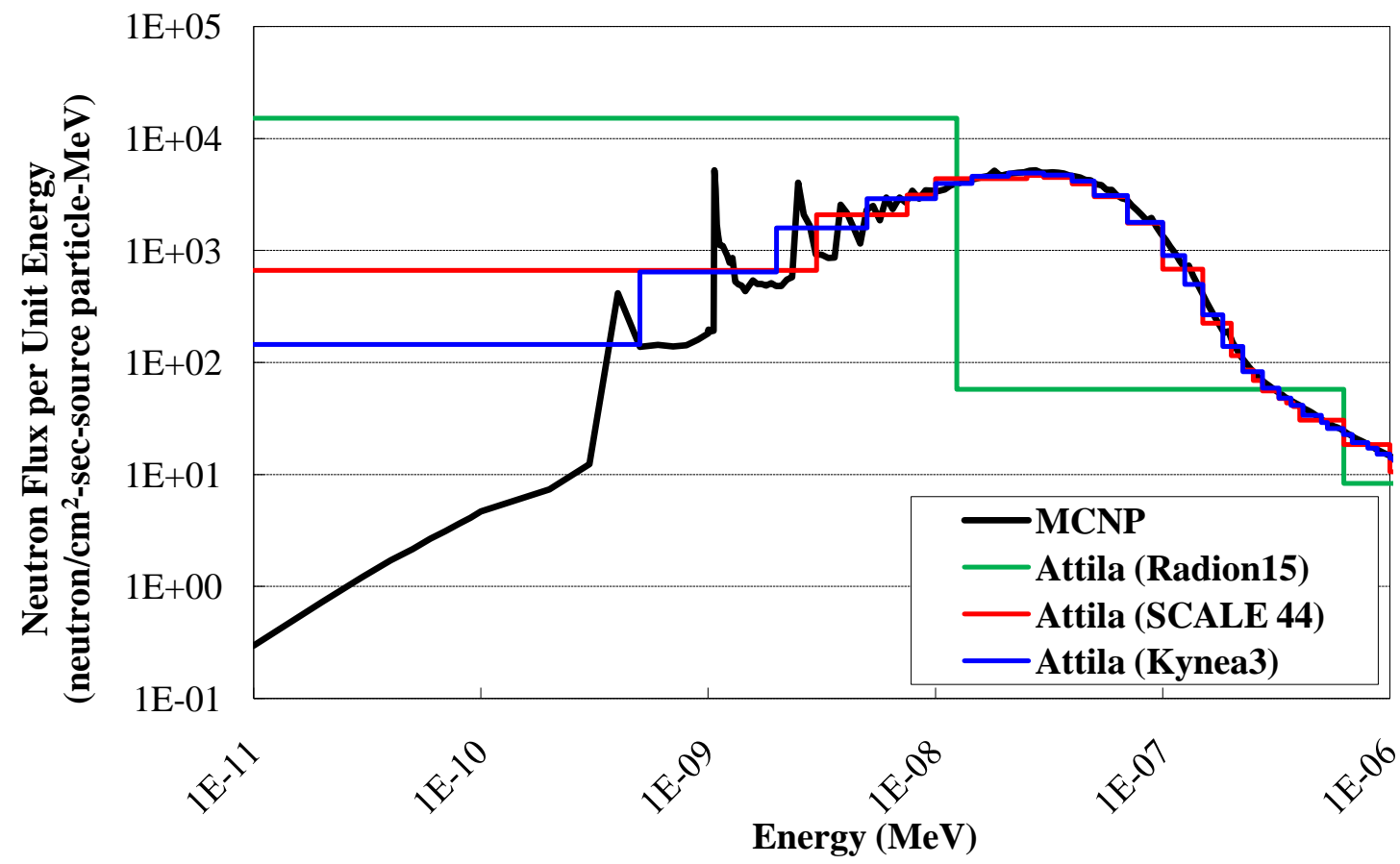

Figure 10 Comparison of Volume-Averaged Thermal Neutron Flux in Polyethylene Cube from MCNP and Attila using Radion15, SCALE44 and Kynea3 Cross-Section Libraries 
The next step is to compare the total photon production cross sections for hydrogen, deuterium and carbon. None of these cross-section libraries contain the required photon production data required to simulate high-resolution gamma-ray spectroscopy, so it will be necessary to generate the photon production cross sections required. For this comparison, NJOY99.259 [3] was used to collapse the ENDF/B-VI photon production cross sections into the group structures from the Radion15, SCALE44 and Kynea3 cross-section libraries for the radiative capture interaction for hydrogen, deuterium and carbon and the inelastic capture interaction for carbon. NJOY99.259, a modular program developed at Los Alamos National Laboratory, is used to convert ENDF/B formatted cross sections into formats used by transport codes [3]. One of the modules of NJOY99.259, GROUPR can be used to generate "self-shielded, multigroup cross sections, group-to-group scattering matrices, photon production matrices, and charged-particle cross sections from pointwise input" [3], GROUPR has the ability to produce photon production matrices from pointwise data based on a user defined neutron and photon energy group structure. An additional NJOY subroutine ACER, which produces a point-wise continuous cross-section file [3], was used in order to examine the behavior of the cross section as a function of energy and to provide comparative data for the multigroup cross sections.

A comparison of the carbon radiative capture and inelastic scatter photon production cross sections are shown in Figure 11 and Figure 12, respectively. The radiative capture cross section for carbon is dominated by the thermal region which is averaged over only three groups in the Radion 15 cross-section library compared to the SCALE44 group and Kynea3 cross-section libraries which contain a large number of 
thermal groups. For the inelastic scattering in carbon, the SCALE44 group cross-section library spans the primary resonance with four energy groups, while the Radion15 and Kynea3 have seven and nine groups, respectively over that resonance. The more energy groups provided by a given cross-section library provide the ability to produce a higherfidelity induced photon spectrum. The larger number of energy groups in both the thermal and high energy ranges provided by the Kynea3 cross-section library allows for higher-fidelity induced photon spectrum for both thermal and resonance-dominated cross sections.

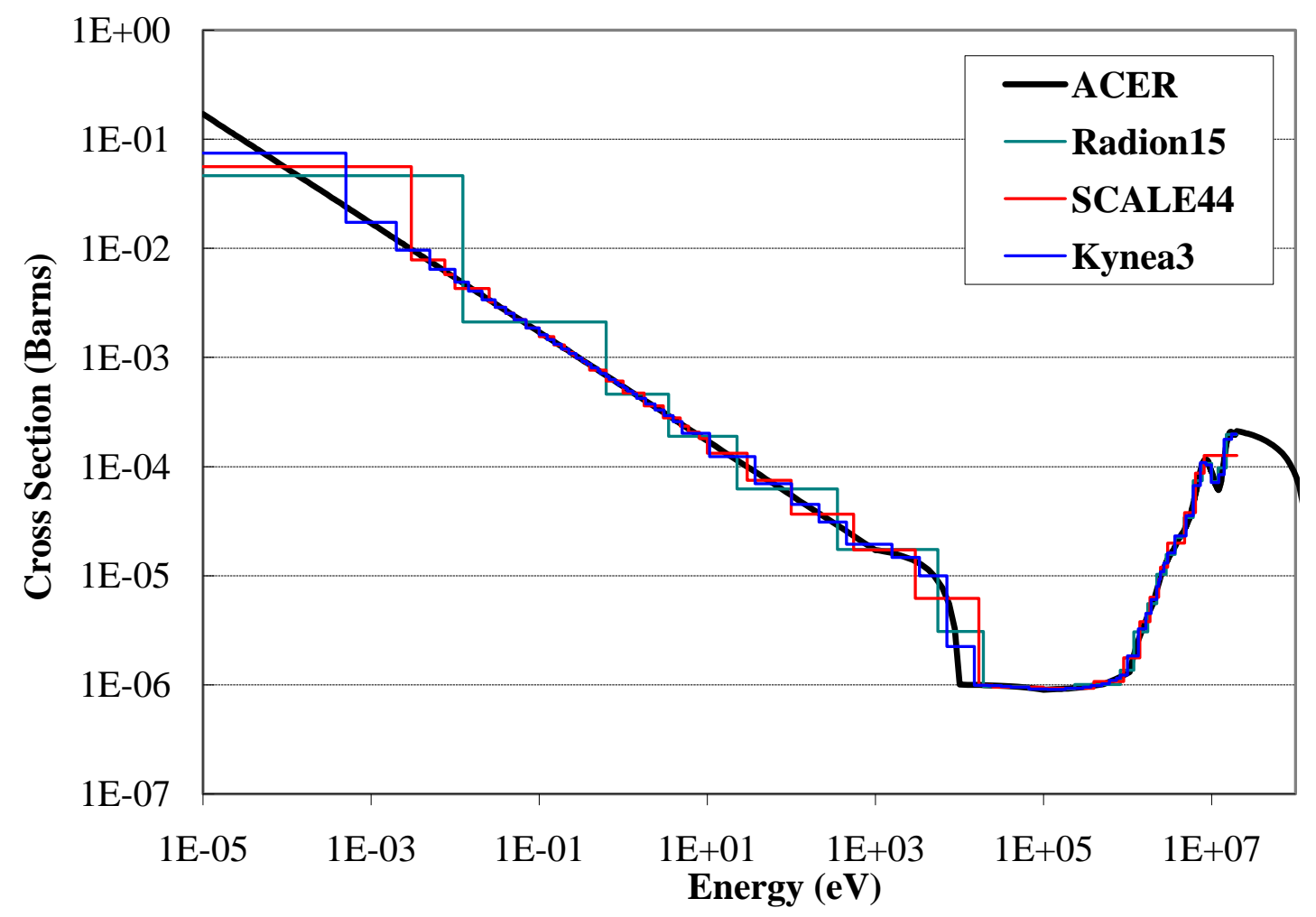

Figure 11. Carbon Radiative Capture Photon Production Cross Section in "Continuous Energy”, Kynea3 Group Structure, SCALE 44 Group Structure and Radion15 Group Structure 


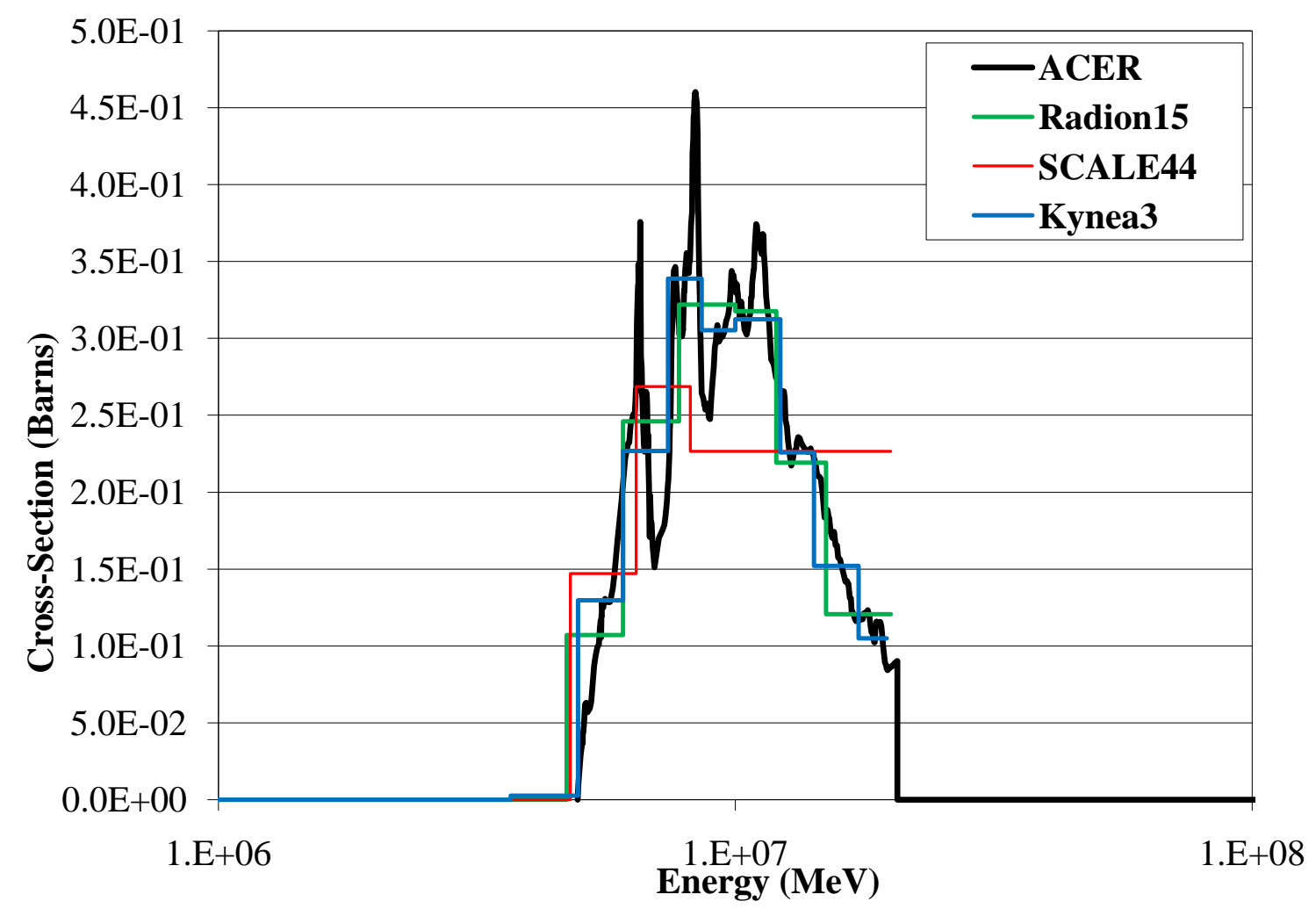

Figure 12. Carbon Inelastic Scattering Photon Production Cross Section in "Continuous Energy”, Kynea3 Group Structure, SCALE 44 Group Structure and Radion15 Group Structure

A more quantitative explanation of the cross-section library choice can be seen by examining reaction rates calculated using each of the photon production cross sections. Using the neutron fluxes calculated using Attila for each cross section set and the photon production cross sections collapsed in NJOY in the appropriate energy group structures, the photon production rates in polyethylene were calculated. MCNP reaction rates were calculated and compared to the calculated photon production rates. This direct comparison of the neutron reaction rate and the photon production rate is possible for polyethylene because the photon yield for these reactions is unity where the photon yield is defined as the ratio of the photon production cross section to the neutron cross section. If the photon yield were not unity for these interactions, a direct comparison of the 
neutron reaction rate and the photon production rate would not produce comparable results. The comparison is shown in Table 2. Although the neutron flux using the SCALE44 and Kynea3 cross-section libraries was within $1 \%$ of the neutron flux obtained from MCNP5, the calculated photon production rates differed by up to $2.25 \%$ for the cross sections collapsed using the Kynea3 bin structure and $4.49 \%$ for the cross sections collapsed using the SCALE44 group bin structure, both for carbon inelastic scatter. The Radion 15 cross-section library overestimates the flux $14.3 \%$ when compared to the MCNP5 calculated neutron flux, because it only contains three upscatter groups and does not contain the $S(\alpha, \beta)$ treatment for polyethylene. For the cross sections collapsed using the Radion15 bin structure, the carbon inelastic scattering photon production rate differed by only $4.5 \%$, while the hydrogen radiative capture photon production rate, which is largely dependent on the thermal neutron flux, differed from MCNP by $76.6 \%$. The difference between the total photon production rate produced by the cross sections collapsed using the Radion15 bin structure and the total MCNP reaction rate was $77.1 \%$. The reaction rate offered by the Kynea3 cross section neutron energy group structure will allow for more accurate generation of the induced photon spectrum, so the Kynea3 crosssection library will be used for neutron transport calculations in coupled neutron-photon simulations. 
Table 2. Reaction Rates Calculated using Attila Flux and Kynea3, SCALE44 and Radion15 Cross Sections and Compared to Reaction Rates Calculated Using MCNP.

\begin{tabular}{|c|c|c|c|c|c|}
\hline & $\begin{array}{c}{ }^{1} \text { H Radiative } \\
\text { Capture }\end{array}$ & $\begin{array}{c}{ }^{2} \text { H Radiative } \\
\text { Capture }\end{array}$ & $\begin{array}{c}\text { C Radiative } \\
\text { Capture }\end{array}$ & $\begin{array}{c}\text { C Inelastic } \\
\text { Scatter }\end{array}$ & Total \\
\hline $\begin{array}{c}\text { MCNP5 } \\
\text { (reactions/sec- } \\
\text { source particle) }\end{array}$ & $4.74 \mathrm{E}-06$ & $8.49 \mathrm{E}-13$ & $2.42 \mathrm{E}-08$ & $1.78 \mathrm{E}-07$ & $4.94 \mathrm{E}-06$ \\
\hline $\begin{array}{c}\text { MCNP Relative } \\
\text { Uncertainty (\%) }\end{array}$ & 0.01 & 0.01 & 0.01 & 0.02 & - --------- \\
\hline $\begin{array}{c}\text { Kynea3 } \\
\text { (reactions/sec- } \\
\text { source particle) }\end{array}$ & $4.67 \mathrm{E}-06$ & $8.37 \mathrm{E}-13$ & $2.39 \mathrm{E}-08$ & $1.82 \mathrm{E}-07$ & $4.87 \mathrm{E}-06$ \\
\hline $\begin{array}{c}\text { SCALE44 } \\
\text { (reactions/sec- } \\
\text { source particle) }\end{array}$ & $4.91 \mathrm{E}-06$ & $8.80 \mathrm{E}-13$ & $2.51 \mathrm{E}-08$ & $1.86 \mathrm{E}-07$ & $5.21 \mathrm{E}-06$ \\
\hline $\begin{array}{c}\text { Radion15 } \\
\text { (reactions/sec- } \\
\text { source particle) }\end{array}$ & $8.37 \mathrm{E}-06$ & $6.51 \mathrm{E}-12$ & $1.89 \mathrm{E}-07$ & $1.86 \mathrm{E}-07$ & $8.75 \mathrm{E}-06$ \\
\hline
\end{tabular}

\subsubsection{Spatial Dependence}

As the neutrons transverse material, interactions occur which alter the neutron spectrum. Due to the dependence of the neutron interaction cross section and the secondary photon energy on the neutron energy, the change in the neutron spectrum will affect the photon flux at the detector location. Since the induced photon spectrum is obtained by multiplying the energy-dependent neutron flux by the multigroup cross sections, the position dependence of the photon spectrum can be determined by examining the variation in the neutron spectrum as a function of position. Theoretically, the neutron spectrum should not change significantly in energy if the distance traveled is less than one mean-free-path, the average distance a particle travels before interacting with the material. A computational mesh can be generated to compare the variation in the neutron spectrum at multiple distances from the source throughout different materials 
to help determine the required level of spatial detail required in the problem. A flux profile for the polyethylene case created using TecPlot [11] is shown in Figure 13. The flux profile graph depicts the log of the flux as a function of position. As shown by scalar flux profile graphs, there is significant flux variation as a function of position within the polyethylene cube. A slice along the $\mathrm{z}$-axis at $\mathrm{z}=0$ is shown in Figure 13.A, which depicts substantial flux variation through the thickness of the cube. Two graphical representations of the flux profile taken from slices along the x-axis are shown in both Figure 13.B and Figure 13.C; B was taken at the center of the cube, and C was taken 1 $\mathrm{cm}$ from the edge closest to the detector. The comparison of the variation in the flux profile diagrams suggests that the spatial and energy dependence of the neutron flux will have an effect on the photon spectrum at the detector location. In order to quantify this effect, a calculation was performed. The volume-averaged scalar neutron flux within entire polyethylene cube was calculated. Additionally, the polyethylene cube was subdivided into 64 equal volumes, and the volume-averaged neutron flux was calculated for each subvolume. Both the volume-averaged flux within the entire polyethylene cube and the volume-averaged flux within each of the subvolumes were used to calculate the induced photon spectrum using the procedure outlined in section 2.2.2. The photon transport calculation was then performed using the procedure outlined in section 2.2.3 to obtain the volume-averaged photon flux within the detector volume. A comparison of the photon flux in the detector calculated using the volume-averaged neutron flux within the entire polyethylene cube with RADSAT, the average neutron flux within each of the subvolumes with RADSAT and a MCNP simulation is shown in Figure 14. A comparison of the flux in each of the photopeaks is given in Table 3. 


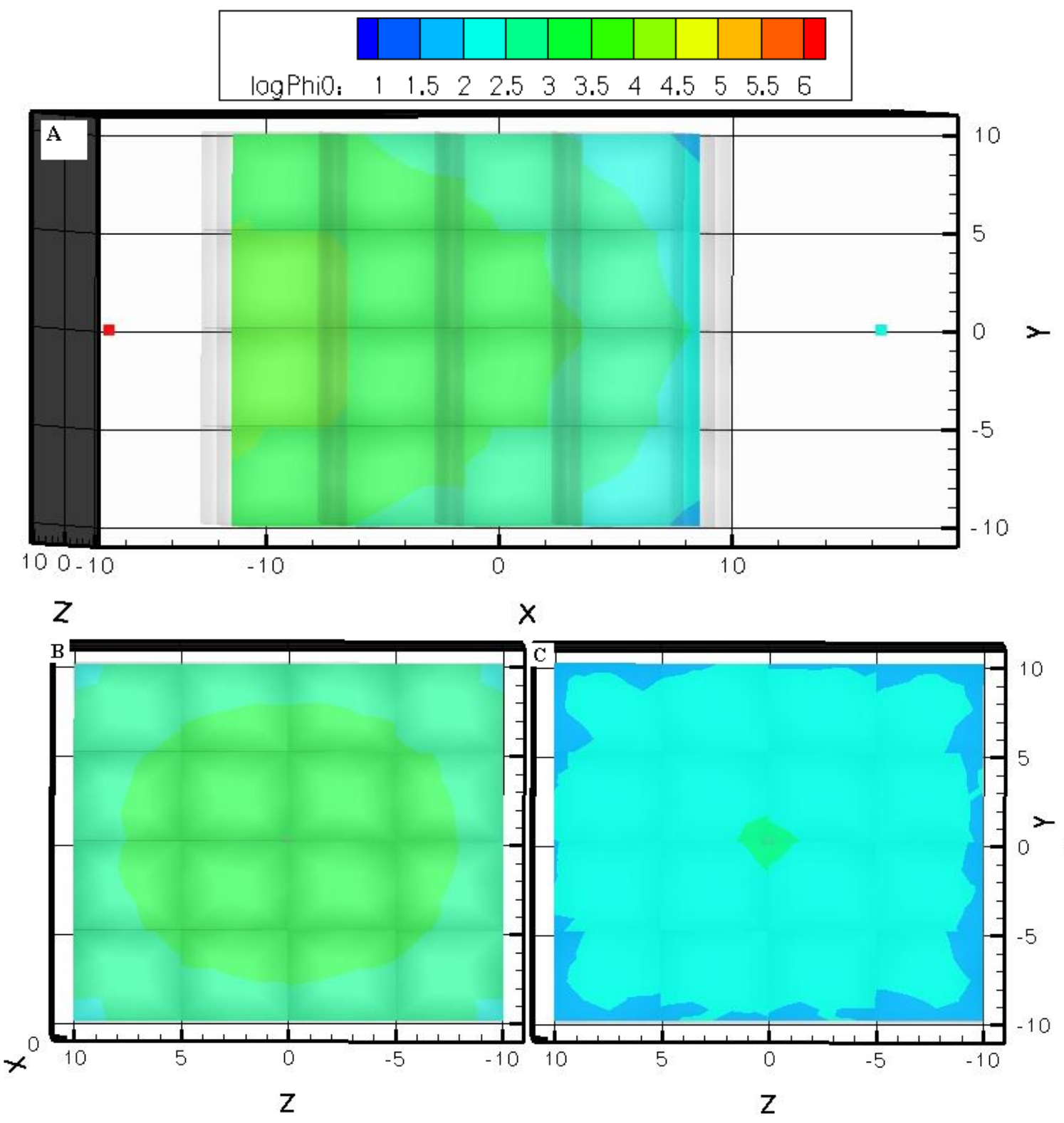

Figure 13. TecPlot of Slices of the Log of the Scalar Flux within the Polyethylene Cube. $\mathrm{A}$ is a slice along the $\mathrm{z}$-axis at $\mathrm{z}=0, \mathrm{~B}$ is a slice along the $\mathrm{x}$-axis at $\mathrm{x}=0$ and $\mathrm{C}$ is a slice along the $x$-axis at $x=9$. 


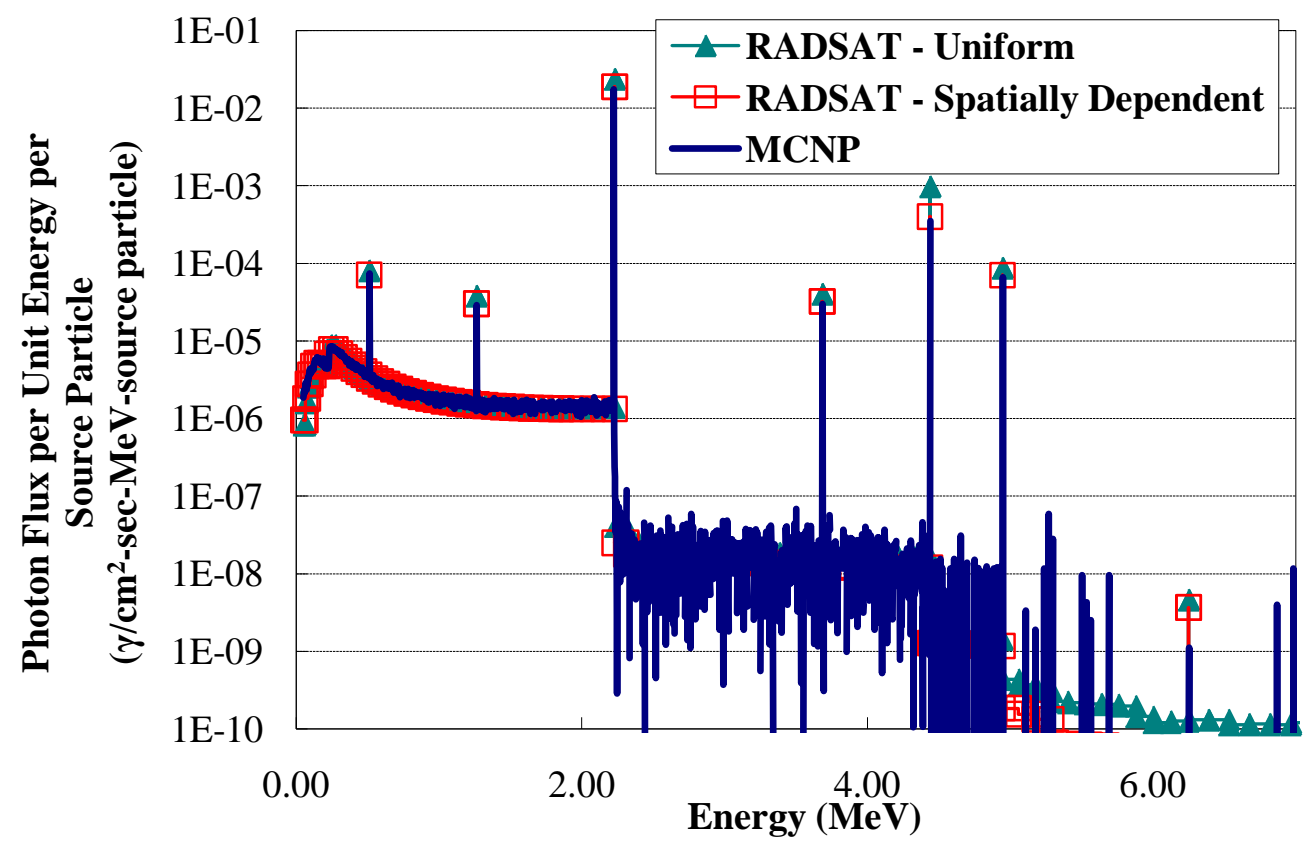

Figure 14. Comparison of Volume-Averaged Photon Flux in Detector for Uniformly Distributed Induced Photon Spectrum, Spatially Dependent Induced Photon Spectrum and MCNP

Table 3. Comparison of the Scalar Flux ( $\gamma / \mathrm{cm}^{2}$-sec-source particle) in Each Photopeak inside the Detector from the Irradiation of Polyethylene

\begin{tabular}{|c|c|c|c|c|c|c|c|}
\hline & \begin{tabular}{|c|}
$\mathrm{C}$ \\
1.2625 \\
$\mathrm{MeV}$
\end{tabular} & $\begin{array}{c}{ }^{1} \mathrm{H} \\
2.223 \\
\mathrm{MeV}\end{array}$ & $\begin{array}{c}\mathrm{C} \\
3.684 \\
\mathrm{MeV}\end{array}$ & $\begin{array}{c}\mathrm{C} \\
4.439 \\
\mathrm{MeV}\end{array}$ & $\begin{array}{c}\mathrm{C} \\
4.9465 \\
\mathrm{MeV}\end{array}$ & $\begin{array}{c}{ }^{2} \mathrm{H} \\
6.25 \\
\mathrm{MeV}\end{array}$ & $\begin{array}{l}\text { Total } \\
\text { Flux }\end{array}$ \\
\hline \begin{tabular}{|l|} 
RADSAT - \\
Uniformly \\
Distributed \\
\end{tabular} & $3.82 \mathrm{E}-08$ & $2.38 \mathrm{E}-05$ & $4.04 \mathrm{E}-08$ & 9.77E-07 & $8.75 \mathrm{E}-08$ & $4.61 \mathrm{E}-12$ & $3.04 \mathrm{E}-05$ \\
\hline $\begin{array}{l}\text { Percent } \\
\text { Difference (\%) } \\
\text { [Uniform and } \\
\text { MCNP] }\end{array}$ & 32.64 & 36.78 & 35.12 & 178.35 & 33.18 & 81.50 & 29.36 \\
\hline $\begin{array}{l}\text { RADSAT - } \\
\text { Spatially } \\
\text { Dependent }\end{array}$ & $3.01 \mathrm{E}-08$ & $1.88 \mathrm{E}-05$ & $3.22 \mathrm{E}-08$ & $4.01 \mathrm{E}-07$ & 7.01E-08 & 3.46E-12 & $2.46 \mathrm{E}-05$ \\
\hline $\begin{array}{l}\text { Percent } \\
\text { Difference (\%) } \\
{[\text { Spatial and }} \\
\text { MCNP] } \\
\end{array}$ & 4.51 & 8.05 & 7.69 & 14.25 & 6.70 & 36.22 & 4.68 \\
\hline MCNP & $2.88 \mathrm{E}-08$ & $1.74 \mathrm{E}-05$ & 2.99E-08 & $3.51 \mathrm{E}-07$ & $6.57 \mathrm{E}-08$ & $2.54 \mathrm{E}-12$ & $2.35 \mathrm{E}-05$ \\
\hline $\begin{array}{l}\text { MCNP Relative } \\
\text { Uncertainty (\%) }\end{array}$ & 2.89 & 0.12 & 2.85 & 0.84 & 1.92 & 100 & 0.18 \\
\hline
\end{tabular}


As Table 3 and Figure 14 show, there was a substantial difference in the calculated photon flux at the detector location when the spatial dependence of the neutron flux was taken into account. When the neutron flux was averaged over the entire polyethylene cube, the total photon flux reaching the detector was much too large. This has to do with the neutron flux distribution shown in Figure 13. By assuming a volumeaveraged neutron flux throughout the entire cube, too many reactions were being located close to the detector. The photons produced by these reactions do not interact in the material causing more of the full energy discrete gamma rays to reach the detector location. This is particularly true for the 4.439-MeV gamma rays from the inelastic scattering interaction in carbon. The threshold for this interaction is approximately 5$\mathrm{MeV}$, and this energy neutron is only present in the region of the polyethylene cube closest to the source. By distributing the photon source uniformly throughout the cube, these gamma rays were produced closer to the detector, artificially increasing the uncollided flux reaching the detector location. The large percent difference between the 6.25-MeV gamma ray produced by the deuterium radiative capture reaction from MCNP and the spatially dependent RADSAT can be attributed to the large statistical uncertainty associated with the MCNP calculation. The log of the spatially distributed photon flux within the cube is shown in Figure 15. 


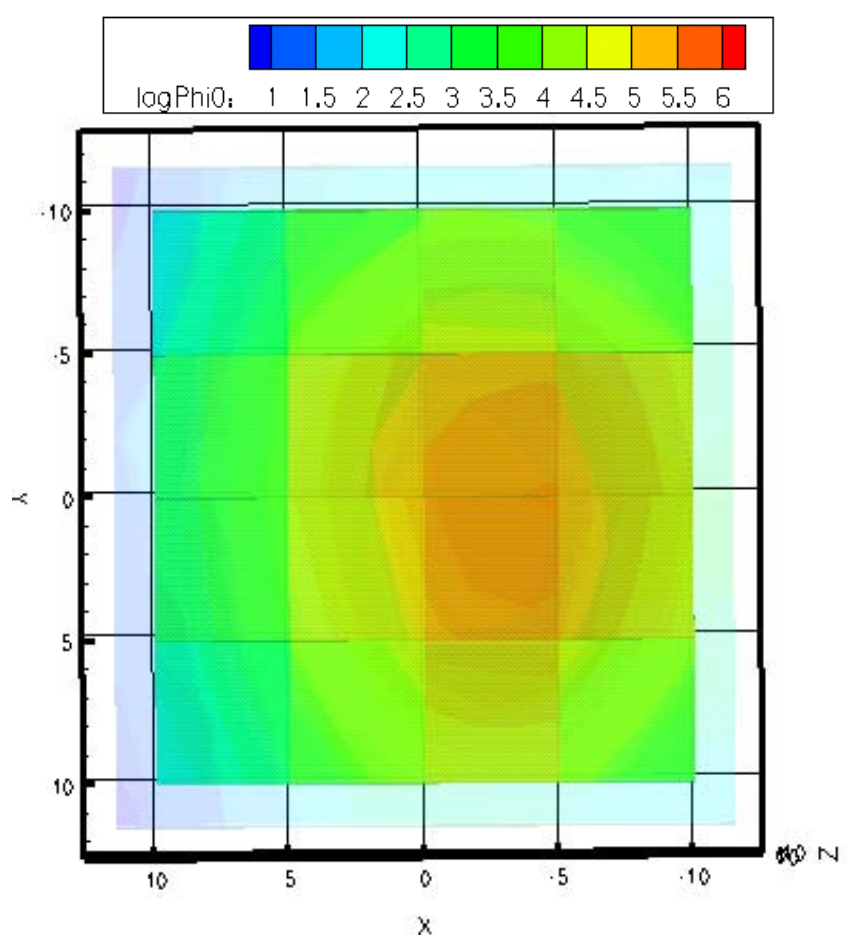

Figure 15. TecPlot of the Scalar Photon Flux within the Polyethylene Cube.

From this comparison it was determined that spatial dependence was necessary to include in the induced photon spectrum source term. It is possible in Attila to map sources to the computational mesh used for the discrete-ordinates calculation, and this method may be required for more complex problems. For the purpose of this project, the spatial dependence was handled by subdividing the volume of the cube into 64 equal subvolumes.

\subsubsection{Neutron Transport Calculation in Attila}

The neutron transport calculations were setup entirely through Attila and currently do not require the use of the RADSAT interface. The standard procedure for completing a discrete-ordinates calculation in Attila was used for these simulations: the problem geometry, cross-section library, and source spectrum must be defined; the transport 
parameters and solution convergence criteria used to solve the problem must be selected; and the output parameters must be defined.

\subsection{Problem Definition}

In order to define the problem setup, three files are provided to Attila: the geometry setup file, the source definition file and the cross section file. For the benchmark cases discussed in this work, the source was defined as a ${ }^{252} \mathrm{Cf}$ Watt-Fission spectrum, and the Kynea3 cross section library was also defined. Attila allows for the geometry of the problem to be defined using CAD (computer-aided design) models. A standard CAD model was created for all of the transport calculations using SolidWorks 2009 SP1, a commercially available CAD program [24]. The same CAD model was to be used for both the neutron and photon transport calculation because the geometry of the simulation cannot be changed between these computational steps. If the geometry of the problem was changed between the neutron transport and photon transport calculations, it could affect the solution to the neutron transport calculation, thus making the source term for the photon transport calculation incorrect. As was determined necessary in Section 2.2.1.2, the moderating cube was subdivided into 64 subvolumes within the CAD model to account for the energy spatial dependence of the neutron flux. Dividing the cube into subvolumes within the CAD model offers some advantages: it allows for easy production of specialized output files containing volume-averaged fluxes and it allows for the easy production of volume-averaged source definitions in later steps.

After the CAD model was imported into Attila, the computational mesh for the problem was created. A global mesh size of 5-cm was defined as the starting place for the iterative process. The mesh generator in Attila was used to determine the 
computational mesh for the problem which contained approximately 7000 cells.

Although the computational mesh was defined here, determining the appropriate computational mesh for a simulation is an iterative process and also a topic of discussion in Section 2.2.1.4.

Once the computational mesh, source and cross section files have been defined, the problem definition can be setup in Attila. First, the materials required in the problem were defined from the materials available in the provided cross-section library. Next, each region of the problem was assigned a material and density. Finally, the region the volume source occupied and the source strength were defined. It should be noted that all sources in both the neutron and photon transport calculations are volume sources in this document. In addition to defining the basic geometry, cross sections and sources necessary to complete the neutron transport simulation, the transport parameters need to be defined in order to complete calculation.

\subsection{Transport Parameters}

The transport parameters define the values required by the discrete-ordinates code to complete the calculation. The transport parameters include the angular quadrature set used discretize the simulation in angle, the scattering treatment, the scatter $\left(\mathrm{P}_{\mathrm{n}}\right)$ and discrete-ordinance $\left(\mathrm{S}_{\mathrm{n}}\right)$ order, and the convergence criteria for the calculation.

The angular quadrature set used to solve the neutron transport determines the approximations used to discretize the problem in angle. There are a number of quadrature sets available in Attila. The Triangular Chebyshev-Lobatto quadrature set, which is used to complete the neutron and photon transport calculations in RADSAT,

places quadrature points mostly uniformly over the unit sphere and contains $n^{2}+2 n-6$ total 
angles, placing a point at each of the poles $(+1$ and -1$)$ [11]. The integration properties of the Triangular Chebyshev-Lobatto quadrature set allow for the exact integration of "the Legendre scattering source up to and including $\mathrm{P}_{2}$ scattering for $\mathrm{S}_{4}$ and up to and including $\mathrm{P}_{3}$ scattering for higher $\mathrm{S}_{\mathrm{n}}$ orders." [11]. Although the other quadrature sets would have provided accurate solutions, the choice of the Triangular Chebyshev-Lobatto quadrature set was made for the neutron and photon transport calculation due to the decreased computational efficiency resulting from the set's integration properties and the uniformity of the distribution of the quadrature points over the unit sphere.

The scattering treatment describes the method used in the computation of the moment-to-discrete matrix. Attila includes two scattering treatments: the standard scattering treatment and the Galerkin scattering treatment. The Galerkin treatment "forces the scattering source to exactly satisfy certain spherical harmonic moment equations" which reduces the inaccuracies that can result from the standard treatment for highly anisotropic scattering [11]. Since RADSAT is designed for problems with a high degree of scattering or attenuation, the Galerkin scattering treatment was used for all of the neutron and photon transport calculations to improve the accuracy of the solution.

The discrete-ordinates $\left(\mathrm{S}_{\mathrm{n}}\right)$ and spherical harmonic scattering $\left(\mathrm{P}_{\mathrm{n}}\right)$ order used by Attila to calculate the transport determine the accuracy of the solution. The $\mathrm{P}_{\mathrm{n}}$ source term accounts for the particle scattering in the problem. The integration of the spherical harmonics functions over the unit sphere, and thus the accuracy of the solution, is a characteristic of the angular quadrature set used to solve the problem. Proper treatment of the $\mathrm{P}_{\mathrm{n}}$ scattering source "requires the use of an angular quadrature set capable of accurately integrating all the spherical harmonics moments" [11]. The $\mathrm{P}_{\mathrm{n}}$ scattering 
source term is limited by the $S_{n}$ order as dictated by the angular quadrature set and scattering treatment used to solve the problem. Although iteration is performed over both the $\mathrm{P}_{\mathrm{n}}$ and $\mathrm{S}_{\mathrm{n}}$ order as will be discussed in Section 2.2.1.4, all of the neutron transport calculations in this document were completed using at least $\mathrm{S}_{16} / \mathrm{P}_{2}$.

The inner iterations, outer iterations and convergence criterion in Attila are user defined transport parameters which determine the convergence of the solution for a particular calculation. The maximum inner iterations specifies the maximum number of iterations within an energy group, and the maximum outer iterations determines the maximum number of allowable outer iterations to account for upscatter within the thermal region. Outer iterations are only necessary in problems where upscatter is present, so the maximum outer iterations for photon transport calculations is one. Both the inner and outer maximum iterations place an upper limit on the number of iterations the transport calculation will perform if the specified convergence criteria is not achieved prior to reaching the maximum. The solution meets the convergence criterion if

$$
\max _{i}\left|\frac{\phi_{i}^{\ell+1}-\phi_{i}^{\ell}}{\phi_{i}^{\ell+1}}\right|<\varepsilon
$$

where $i$ is the cell index, $\phi_{i}$ is the cell averaged flux, and $\ell$ is the iteration number [11]. For both neutron and photon transport calculations, the maximum inner iterations and the convergence criteria were set at 100 and $1 \times 10^{-4}$, respectively which are both default values in Attila. The maximum number of outer iterations for the neutron transport calculations was 200. The number of maximum outer iterations was set to 200 to ensure 
that the calculation did not terminate prior to converging. These parameters were used for all of the deterministic transport calculations in this document.

\subsection{Output Parameters}

Although Attila solves the problem globally, specific outputs called reports were required to complete the induced photon calculation. Due to the required spatial dependence of the neutron flux, a specific formatting for the reports was required in order to efficiently complete the calculation. Since the spatial dependence of the problem is handled by subdividing the volumes in the CAD model, it is necessary to obtain the volume-averaged scalar flux in each of the subvolumes. Additionally a report containing the volume-averaged scalar flux within the entire cube was required. The volumeaveraged scalar flux for each subvolume was used for all induced photon calculations, and the volume-averaged scalar flux over the entire cube was used for diagnostic purposes only.

\subsubsection{Discrete Ordinates Solution Accuracy}

Determining the solution convergence of the deterministic transport calculation is an iterative process. Refinement of the mesh, discrete ordinance order and scattering order should be completed to determine how the solution is changing. A numerical solution convergence is reached when the simulated solution approaches an asymptotic value. The numerically converged solution is the answer to the problem being simulated. The consistency and stability of the numerical methods employed by Attila provide a strong foundation for the neutron transport calculations. Attila employs the discontinuous, finite-element spatial discretization (DFEM) which has been shown to be 
third-order accurate for integral quantities (e.g. volume-averaged flux within a material) and second-order accurate for pointwise quantities (e.g. scalar flux at the detector location) on unstructured (tetrahedral) grids [25][26]. Additionally, the unstructured tetrahedral mesh implemented in Attila ensures that local spatial errors do not propagate throughout the entire solution [11].

The first step in the refinement process was to determine if the computational mesh has been spatially resolved. For coupled neutron-photon problems, it was important that the neutron flux solution contains no negative values and that the solution was resolved throughout the entire problem in order to properly produce the induced photon spectrum. Negative flux values can be a sign that the mesh needs to be refined in specific regions. It was also important to examine the solution for discretization errors. The flux was then visualized over the entire problem to help diagnose any obvious modeling errors or large discretization effects such as ray effects. Once the solution is examined for negative values and discrization errors, the mesh size is iterated on. This procedure was repeated several times in order to determine the appropriate mesh to use for the problem.

The second step in determining solution convergence was refinement of the discrete-ordinates $\left(\mathrm{S}_{\mathrm{n}}\right)$ and scattering $\left(\mathrm{P}_{\mathrm{n}}\right)$ order. An iterative process was used to determine convergence based on the $S_{n}$ order. Initially, a low $S_{n}$ order was used to solve the problem. Then the $S_{n}$ order was increased until the solution stops changing. In addition to the $S_{n}$ order, the spherical-harmonic $\left(P_{n}\right)$ scattering source expansion also determines the accuracy of the solution. An iterative process was also used to determine solution accuracy based on the $\mathrm{P}_{\mathrm{n}}$ order. The $\mathrm{P}_{\mathrm{n}}$ order was increased iteratively within the 
bounds dictated by the angular quadrature set for a given scattering treatment and $S_{n}$ order. This procedure was repeated several times in order to determine the appropriate $S_{n}$ and $\mathrm{P}_{\mathrm{n}}$ order to use for the problem.

The same solution convergence criteria were applied to all photon transport calculations as were applied to the neutron transport calculations in this document. All the solutions were examined for negative values and a three-dimensional visualization tool was used to check for anomalies. Additionally, iterations were performed on $S_{n}$ and $\mathrm{P}_{\mathrm{n}}$ order to determine the appropriate transport parameters for the calculation. The coupled neutron-photon calculation required that the same computational mesh be used for both the neutron and photon transport calculations. Since the neutron transport calculation places more stringent requirements on the mesh, the mesh iterations were only completed for the neutron transport calculations

\subsubsection{Generation of Neutron-Induced Photon Spectrum}

The second step in simulating a prompt gamma neutron activation problem using a coupled deterministic-Monte Carlo method is the generation of the neutron-induced photon spectrum. The neutron flux obtained from the Attila neutron transport simulation was used to calculate the photon production rate in a material using Equation 2.2.

$$
R\left(E_{\gamma}, \vec{r}\right)=\sum_{g=1}^{G} N_{i} \sigma_{(n, \gamma)}^{i}\left(E_{g} \rightarrow E_{\gamma}\right) \phi_{g}(\vec{r}) \quad \text { Equation } 2.2
$$


where $\mathrm{N}_{i}=$ number density of element or isotope $\mathrm{i}$

$\sigma_{(n, \gamma)}^{i}\left(E_{g} \rightarrow E_{\gamma}\right)=$ energy dependent microscopic neutron cross section for a reaction producing a gamma-ray of element or isotope $i$

$\varphi_{g}(\vec{r})=$ energy and spatial dependent neutron flux

$\mathrm{R}\left(E_{\gamma}, \vec{r}\right)=$ photon production rate

The existing multigroup, coupled neutron-photon cross-section libraries are not typically used for the simulation of high-resolution gamma-ray spectroscopy. Existing multigroup, neutron-photon cross-section libraries (i.e. Radion 15 cross-section library) are typically used for photon dose rate and total flux calculations rather than highresolution gamma-ray spectroscopy. Consequently, existing cross-section libraries generally consist of a relatively few (i.e. 10-20) broad photon groups spanning energies from approximately 0.01 up to several-MeV. The broad energy groups available in current coupled neutron-photon cross-section libraries are unsuitable for the simulation of high-resolution gamma-ray spectroscopy, which requires the preservation of discreteenergy photons in order to simulate lines in the spectrum.

In addition to the energy group structure, the current format of coupled neutronphoton cross-section libraries is not suitable for high-resolution gamma-ray spectroscopy applications. Existing coupled neutron-gamma cross-section libraries combine the crosssections for discrete and continuum gamma productions into one multigroup gamma production matrix. This means that the production rate for the characteristic discreteenergy emissions cannot be calculated separately from the continuum. Since the continuum of the gamma-ray spectrum is a smoothly varying function, a coarse energy group structure can be used to sufficiently describe it if the discrete gamma-ray 
production rates can be separated from the production rates of the continuum. Using the current cross-section libraries for the calculation, the discrete gamma-ray production rates will be smeared into broad energy groups with the continuum. A specially formatted multigroup coupled neutron-photon cross-section library was developed for highresolution gamma-ray spectroscopy applications.

\subsubsection{Photon Production Cross-Section Library Development}

In order to simulate high-resolution gamma-ray spectroscopy using a coupled deterministic-Monte Carlo method, a specialized gamma production cross section set was developed, RADSAT-NG, which overcomes the typical shortcomings of using multigroup cross sections to complete these calculations. The unique feature of RADSAT-NG is that it supports the separate calculation of discrete and continuum photons, on a reaction-byreaction basis, from a multigroup neutron flux. The partitioning of the discrete and continuum components of the photon production cross section results in a uniquely formatted multigroup cross section library. The RADSAT-NG cross section library contains a multigroup neutron cross-section for the production of each of the discrete gamma rays and a matrix of multigroup neutron-photon cross-sections for the production of photons in the continuum. This separation allows the continuum cross-section to be expressed in coarse energy groups and the discrete energy gamma rays to be produced and transported at their exact energy (or within a very narrow energy group).

Due to the necessity to partition the continuum and discrete photon production cross sections, it was necessary to develop a methodology for the generation of the cross section set. Initially, the use of an existing cross-section preparation code that allows the user some flexibility in terms of energy group structure, in particular the GROUPR 
subroutine of NJOY [3], was explored. Unfortunately, GROUPR does not allow sufficient flexibility in photon group structure to achieve narrow energy bins about key discrete-energy emissions. Additionally, GROUPR does not differentiate between a discrete and continuum gamma ray produced by the same interaction. For these reasons, NJOY GROUPR was not able to produce suitable cross-section libraries in this work. Since existing cross section production methods were not capable of creating multigroup neutron photon cross section that partition the continuum and discrete photon production cross sections, a method for developing the RADSAT-NG cross section library was developed.

The procedure used for producing the RADSAT-NG coupled neutron-photon cross-section library was completed using a two step process:

1. The application of Doppler broadening,

2. The creation of point-wise cross sections (ACER format) from the raw crosssection data in the ENDF/B format,

3. The collapsing of point-wise data into multigroup neutron cross sections for the production of discrete gamma rays and a neutron-gamma production matrix for the production of continuum gamma rays.

The creation of point-wise cross sections from the raw cross-section data in the ENDF/B format and the application of Doppler broadening were performed using modules within NJOY. A breakdown of each of the NJOY modules used and the functions it performed is provided in APPENDIX D and an example input file for NJOY to produce the pointwise "continuous" hydrogen is shown in APPENDIX E. The collapsing of the point-wise 
cross section data was then performed using the RADSAT-NG cross-section collapsing procedure.

\subsection{RADSAT-NG Cross Section Collapsing Procedure}

Starting from the ACER file produced using NJOY, it was first necessary to parse the data to prepare for the cross section collapsing. Since only the photon production cross sections were generated, only specific sections of the ACER file were required: the neutron cross sections, the photon production yields, photon production cross sections, and photon energy distributions. Each of these data structures were associated with a unique energy set: the neutron cross sections and the photon production cross sections were on the same neutron energy grid; the photon production yields were associated with coarser neutron energies than the neutron cross sections; and the photon energy distributions were associated with coarse neutron energies and with photon energies. The angular dependence data for the photon production was not parsed from the ACER file because isotropic photon production was assumed for all RADSAT-NG cross sections. Of the ten isotopes considered in this document, seven of them only have isotropic photon production. The remaining three have a limited number of photons produced anisotropically. Since RADSAT is intended for use in problems dominated by scattering and attenuation and many photon production interactions are isotropic, the anisotropic production of the induced photons should not drastically affect the photon flux at the detector location.

Once the ACER file was parsed, it was necessary to convert similar data into the same form. The photon production yields and the photon production cross sections contain the same data in a slightly different format. The photon production yields were 
converted to photon production cross sections using Equation 2.3. Since the photon production yields were not represented on the same neutron energy grid as the neutron cross sections, linear interpolation was used to extrapolate the yields onto the same neutron energy grid as the neutron cross section.

$$
\begin{gathered}
\sigma_{(n, \gamma)}\left(E_{n}\right)=\sigma_{n}\left(E_{n}\right) y\left(E_{n} \rightarrow E_{\gamma}\right) \\
\text { where } y\left(E_{n} \rightarrow E_{\gamma}\right)=\frac{\sigma_{(n, \gamma)}\left(E_{n}\right)}{\sigma_{n}\left(E_{n}\right)}=\text { the photon production yield } \\
\sigma_{(n, \gamma)}\left(E_{n}\right)=\text { the photon production cross section } \\
\sigma_{n}\left(E_{n}\right)=\text { the neutron cross section }
\end{gathered}
$$

In preparation for the neutron group collapsing, it was necessary to add the neutron energies associated with the Kynea3 neutron group boundaries to the energy grid for both the photon production cross sections and the neutron cross sections. Since the energy grid for the photon production cross sections and the neutron cross sections was the same, the energy grid was searched to determine if it contained the Kynea3 neutron group boundaries. The neutron group boundaries that were not included were added to the energy grid. The photon production cross sections and the neutron cross sections for the added neutron group boundaries were calculated using linear interpolation.

Next the discrete photon production cross sections were separated from the continuum photon production cross sections. Interactions were broken down into many "sub-reactions", one for each possible discrete gamma ray that the interaction can produce and one for the continuum. Using the photon energy distributions, the "subreactions" were identified as either discrete or continuum. Once identified the discrete 
photon production cross sections were stored in one array while the continuum photon production cross sections were stored in a separate data structure. This ability to obtain a cross section for each individual discrete gamma ray and one for the continuum produced by a single interaction was the cornerstone of the RADSAT-NG cross-section library. In addition to distinguishing between discrete and continuum photon production cross sections, the photon energy distribution contained probability density functions for the production of the continuum data. In order to produce the 400 continuum groups, it was necessary to interpolate the probability density function for the continuum production to include the (401) photon energy group boundaries. 400 continuum photon groups were chosen since this allowed for equal-width energy bins (50-keV) ranging from 0 to $20-\mathrm{MeV}$. Since the probability density functions were provided in a stepwise data format as a function of photon energy, the probability density function values were assumed to be uniform within each energy group, thus linear interpolation was not used when adding the photon energy group boundaries. Next the probability density functions were interpolated over the photon production cross section neutron energy grid.

Although linear interpolation was not used when adding photon energy group boundaries to the photon production cross section neutron energy grid, it was used when adding neutron energy group boundaries. Finally, the probability matrix was computed using Equation 2.4.

$$
\begin{aligned}
p\left(E_{n} \rightarrow E_{\gamma}\right) & =\int_{E_{g}}^{E_{g-1}} f\left(E_{n} \rightarrow E_{\gamma}\right) d_{\gamma} \\
& \cong \sum_{E_{g}}^{E_{g-1}} f\left(E_{n} \rightarrow E_{\gamma}\right) \Delta E_{\gamma}
\end{aligned}
$$


where $f\left(E_{n} \rightarrow E_{\gamma}\right)=$ continuum photon production probability density function $\mathrm{p}\left(E_{n} \rightarrow E_{\gamma}\right)=$ the probability that an incident neutron with energy $E_{n}$ will produce a continuum gamma ray with energy $E_{\gamma}$

The appropriate neutron flux approximations were then determined for each neutron energy point on the photon production cross section energy grid. The neutron flux approximation used for the collapsing was approximated as a Maxwellian in the thermal region $\left(1 \times 10^{-6}-\mathrm{eV}\right.$ to $\left.100-\mathrm{eV}\right), 1 / \mathrm{E}$ in the epithermal region $(100-\mathrm{eV}$ to $50-\mathrm{keV})$, and a Watt-fission spectrum in the fast region (50-keV to 20-MeV) [27]. The reason for this flux approximation is that the anticipated neutron flux seen in many of the intended applications for the RADSAT-NG cross-section library is some variation of a fission spectrum. The source used in current applications and the benchmark cases in this document utilized a ${ }^{252} \mathrm{Cf}$ source. Some experimentation was done to determine the effect of collapsing the cross section using a different flux approximation.

The resonance self-shielding effect was then estimated for both the collapsing of the discrete and continuum cross sections using the Bondarenko method [28] as shown in Equation 2.5

$$
\phi^{i}=\frac{\omega_{n}(E)}{\sigma_{t}^{i}(E)+\sigma_{0}^{i}(E)}
$$

where $\phi^{i}$ is the scalar flux for the nuclide $\mathrm{i}, \omega_{n}(E)$ is the neutron flux approximation, $\sigma_{t}^{i}(E)$ is the total microscopic neutron cross section for the nuclide i, and $\sigma_{0}^{i}$ is the cross section for all other nuclides in the mixture excluding nuclide i per atom of the 
nuclide $\mathrm{i}$ or background cross section [29]. In the Bondarenko model the flux is assumed to vary inversely to the total macroscopic cross section. When resonances are present in the total cross section, it produces dips in the corresponding flux profile. The background cross section allows for the cross section to be calculated without knowing exactly what mixtures of the isotope will be used in by assuming a constant within a given neutron energy group. A background cross section of $1 \times 10^{10}$ was used to create the RADSATNG cross sections, which closely approximates an infinitely dilute system. Using the neutron energies grid associated with the photon production cross sections, an array containing the Bondarenko factor was created to use in the cross section collapsing.

Next the point-wise cross-sections for the discrete gamma rays were collapsed into the neutron energy group structure of the Kynea3 cross-section library using Equation 2.6.

$$
\begin{aligned}
& \sigma_{\left(n, \gamma_{-} \text {discrete }\right)}^{g} \frac{\int_{E_{g}}^{E_{g-1}} d E_{n} \sigma_{\left(n, \gamma_{-} \text {discrete }\right)}\left(E_{n} \rightarrow E_{\gamma}\right) \phi_{n}(E)}{\int_{E_{g}}^{E_{g-1}} d E_{n} \phi_{n}(E)} \\
& \cong \sum_{E_{g}}^{E_{g-1}} \sigma_{\left(n, \gamma_{-} \text {discrete }\right)}\left(E_{n} \rightarrow E_{\gamma}\right) \phi^{i}(E) \Delta E_{n} \\
& \sum_{E_{g}}^{E_{g-1}} \phi^{i}(E) \Delta E_{n}
\end{aligned}
$$

where $\sigma_{\left(n, \gamma_{-} \text {discrete }\right)}\left(E_{n} \rightarrow E_{\gamma}\right)=$ microscopic cross-section for a neutron with energy $E_{n}$ to produce a discrete gamma ray with energy $E_{\gamma}$ $\phi_{n}(E)=$ energy dependent neutron flux 
Then the continuum production was collapsed into the neutron-induced gamma production matrix using Equation 2.7. The continuum was collapsed into the neutron energy group structure of the Kynea3 cross-section library and 400 equal-width photon energy bins ranging from 0 to $20-\mathrm{MeV}$. Additionally, the lower limit threshold for collapsing continuum cross sections was set such that if the maximum continuum photon energy produced was less than or equal to $1-\mathrm{keV}$ the cross section was not collapsed. This limit was set to eliminate spending computational time collapsing cross sections that would not contribute to the detector response.

$$
\begin{aligned}
\sigma_{\left(n, \gamma_{-} \text {continuum }\right)}^{g}= & \frac{\int_{E_{g}}^{E_{g-1}} d E_{\gamma} \int_{E_{g^{\prime}}}^{E_{g^{\prime}-1}} d E_{n}^{\prime} \sigma_{\left(n, \gamma_{-} \text {continuum }\right)}\left(E_{n}^{\prime} \rightarrow E_{\gamma}\right) p\left(E_{n}^{\prime} \rightarrow E_{\gamma}\right) \phi_{n}\left(E^{\prime}\right) \phi_{\gamma}(E)}{\int_{E_{g}}^{E_{g-1}} d E_{\gamma} \int_{E_{g^{\prime}}}^{E_{g^{\prime}-1}} d E_{n}^{\prime} \phi_{\gamma}(E) \phi_{n}(E)} \\
\cong & \frac{\sum_{E_{g}}^{E_{g-1}} \sum_{E_{g^{\prime}}}^{E_{g^{\prime}-1}} \sigma_{\left(n, \gamma_{-} \text {continuum }\right)}\left(E_{n}^{\prime} \rightarrow E_{\gamma}\right) p\left(E_{n}^{\prime}, E_{\gamma}\right) \phi^{i}\left(E^{\prime}\right) \omega_{\gamma}(E) \Delta E_{n}^{\prime} \Delta E_{\gamma}}{\sum_{E_{g}}^{E_{g-1}} \sum_{E_{g^{\prime}}}^{E_{g^{\prime}-1}} \phi^{i}\left(E^{\prime}\right) \omega_{\gamma}(E) \Delta E_{n}^{\prime} \Delta E_{\gamma}}
\end{aligned}
$$

where $\sigma_{\left(n, \gamma_{-} \text {continuum }\right)}\left(E_{n} \rightarrow E_{\gamma}\right)=$ the microscopic cross section for a neutron with energy $E_{n}$ to produce a continuum photon with energy $\mathrm{E}_{\gamma}$ $\phi_{\gamma}(E)=$ energy dependent photon flux $\omega_{\gamma}(E)=$ photon flux approximation

The gamma-ray flux for the collapsing was approximated as uniform over the entire energy range since the continuum photon flux should not change significantly within a particular energy group. This assumption is supported by the fact that photon cross-sections above the x-ray absorption edges are smooth and without resonance 
features. Additionally, the continuum energy groups in RADSAT-NG are defined to be relatively narrow (generally less than $50-\mathrm{keV}$ ).

\subsection{Photon Production Cross-Section Library Format}

The organization of the photon production cross section is needed to support efficient calculations of the neutron-induced photon spectrum. In order to reduce the number of continuum calculations completed, thus reduce the computation time, the photon continuum production cross sections for all interactions for a given isotope were combined to form one photon production matrix for the production of all continuum photons. A calculation for the production of each discrete energy gamma ray was completed individually. The first entry in the cross section data file for a given isotope is the isotope identification number or ZAID. The first block of data preceding the ZAID contains the discrete energy gamma-ray cross sections for the isotope. The discrete energy is given in units of-MeV and followed by the multigroup neutron cross section for the discrete gamma ray. The final block of data contains the continuum photon production matrix. An example of a gamma production cross section file is shown in APPENDIX F.

\subsection{Photon Production Cross-Section Library Benchmarking}

Ideally, the RADSAT-NG cross-sections would be benchmarked against existing cross-sections on a reaction-by-reaction, discrete-line by discrete-line basis. Lacking an equivalent line-by-line cross-section set, the basis for benchmarking of RADSAT-NG becomes a comparison to existing multigroup cross-section sets, using the sum over all reactions, and over all discrete and continuum photon production. In order to have a 
point of comparison, the GROUPR module in NJOY was used to produce multigroup photon production. An example input file for NJOY using the GROUPR subroutine is shown in APPENDIX G. Comparisons of the RADSAT-NG and GROUPR photon production cross sections will be made in the RESULTS.

\subsubsection{Neutron-Induced Photon Spectrum Calculation}

Currently the calculation of the neutron-induced photon spectrum is completed using a Visual Basic script external from the RADSAT interface. It processes the region dependent neutron flux output file generated from the neutron transport calculation and the required RADSAT-NG cross-section file. A separate calculation must be completed using Equation 2.2 for each isotope in the simulation, and the user must specify the number density for each isotope in each spatial region of the simulation. The current output is a separate text file, called a "pseudo-isotope" file, for each isotope in each region of the simulation. For example, the polyethylene cube case produces 194 text files to be used as source files for RADSAT since there are three isotopes and 64 photon production regions in the simulation. In RADSAT a "pseudo-isotope" is defined as a commonly used spectrum, such as the cosmic ray spectrum, which is not an isotope but does have a well-defined spectrum containing discrete photons, continuum photons, or both. Although the induced photon spectrum are not commonly used spectrum like a cosmic ray spectrum, the format of the "pseudo-isotope" file allows for the definition of the induced photon spectrum as an input to RADSAT. An example "pseudo-isotope" file for an induced photon spectrum from the neutron irradiation of phosphorus is shown in APPENDIX $\mathrm{H}$ and contains both a discrete and continuum section. In the future these 
calculations will be completed in the RADSAT interface, and the number densities will be obtained from the Attila neutron calculation.

\subsubsection{Photon Transport}

The third step in completing a coupled neutron-photon calculation for the simulation of high-resolution gamma-ray spectroscopy applications was to complete the photon transport calculation. The photon transport problem was completed in two steps: the preprocessing step which was completed through the RADSAT interface and the photon transport calculation which was completed using Attila. Both of these steps were part of the previously defined RADSAT method. While these methods were utilized heavily in this work, no modifications to the existing methodology were required in order to accommodate this new class of problems.

\subsubsection{RADSAT Interface Process}

For the coupled neutron-photon calculations, the RADSAT interface was used to simplify the source definition, define the photon energy group structure, and to produce the photon cross section library to be used in Attila.

The first step was to define the sources to be used in the Attila photon transport calculation. The isotope and region dependent induced photon spectrum calculated in the Section 2.2.2.2, in the form of "pseudo-isotope" files, were used as the photon source terms in RADSAT. A source was defined in RADSAT for each spatial region in the problem, and each induced photon spectrum was assigned to the appropriate source in RADSAT based on the spatial region in which it was produced. This combines all the 
isotopes present in a specific region, so there are only two volume sources for each region in Attila, one source containing all of the discrete lines and one containing the continuum.

The second step was to determine the photon energy group structure for the photon transport calculation. The photon spectrum for each neutron-induced photon simulation is unique. Every isotope has its own unique signature, so defining a predetermined photon group structure to the photon spectrum would not allow the simulation to maintain as much detail in the gamma-ray spectrum throughout the calculation. For this reason, the photon energy group structure for the gamma problem was defined using the adaptive approach built into the RADSAT interface [1]. The upper and lower photon energy threshold, the energy weighting factor, the number of discrete photons to be included in the calculation and the number of continuum groups to be included in the calculation were defined. To preserve the important gamma-ray lines, narrow "peak" groups (typically $0.5-\mathrm{keV}$ or less) were centered around the most prominent emission energies in the problem. All lower-yield discrete emissions, and any continuum source terms were then distributed into broader continuum groups that fill the energy range between peak groups. The visualization tool in RADSAT was used to iterate on the photon group structure until an appropriate group structure was created for each benchmarking problem in this document. An example of the graphical display of the RADSAT group structure for iron is shown in Figure 16. For the iron case, 149 0.3$\mathrm{keV}$ wide peak groups, including one for the $511-\mathrm{keV}$ annihilation peak, and 150 nonpeak groups were used. A $1 / \sqrt{\boldsymbol{E}}$ energy weighting factor was applied to continuum groups to make finer groups in the lower energy region and coarser energy groups in the higher energy range. 


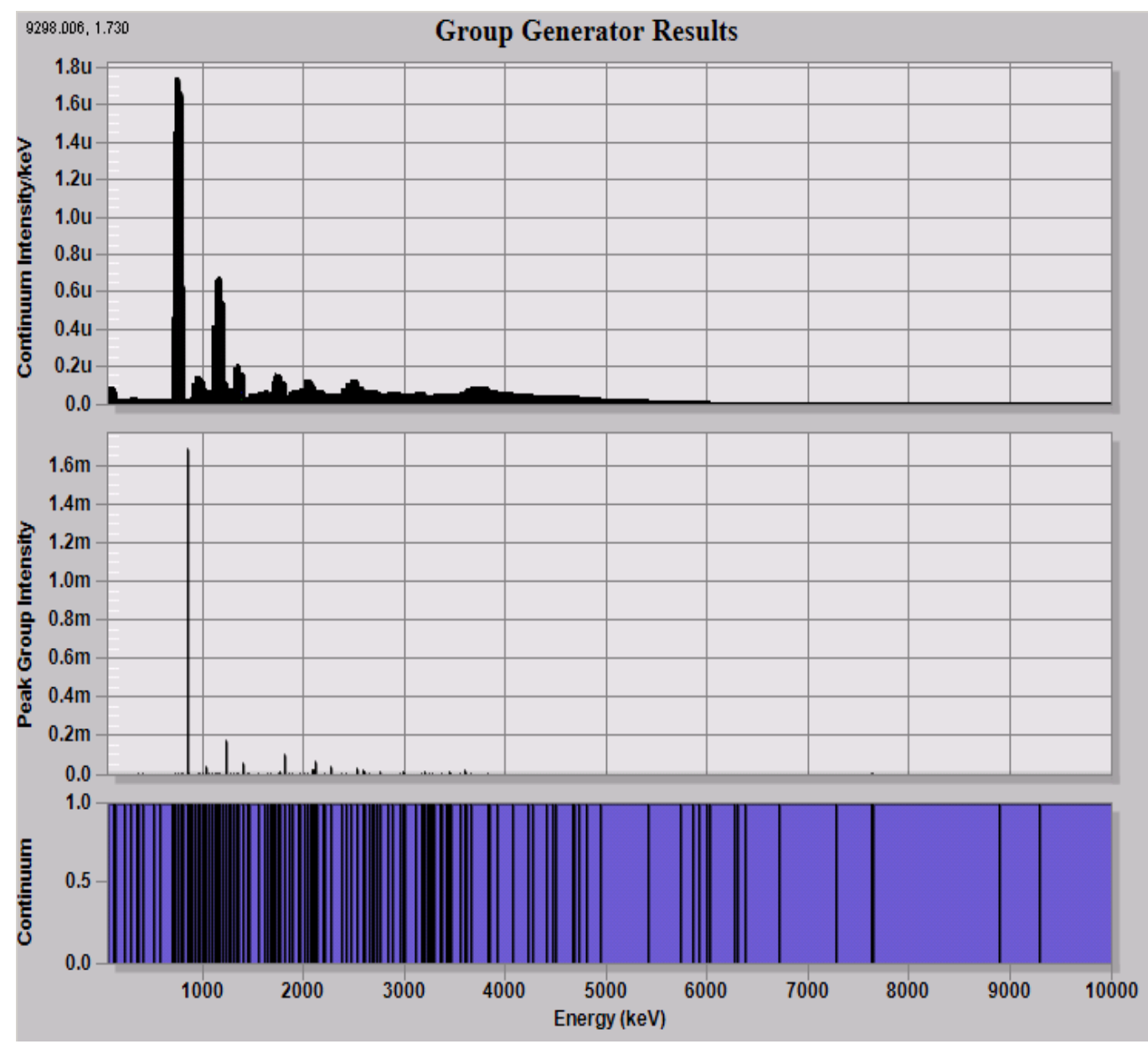

Figure 16. Adaptive Algorithm Applied to Neutron-Induced Photon Spectrum from the Irradiation of Iron

Once the energy group structure was determined using this adaptive algorithm, the group structure was used to generate a problem-specific photon cross-section library using CEPXS [30]. CEPXS produces macroscopic multigroup photon cross sections for a specified material at a particular density [30]. The CEPXS cross section file and the augmented source files in the new group structure were then used in the Attila photon transport calculation. 


\subsubsection{Attila Photon Transport Calculation}

The RADSAT photon transport approaches are a combination of analytical raytracing, integral transport, and discrete ordinates methods - an approach capable of mitigating ray effects for many radiation-detection scenarios. The result of these calculations is a multigroup, discrete ordinates angular flux at all points in the problem. The angular flux can then be used for subsequent pulse-height tally calculations at the detector location.

In order to complete the photon transport, the same procedure was used as was applied for the neutron transport calculation. The CEPXS cross-section library and the regional dependent neutron-induced photon spectrum source terms were used for the photon simulations. The material must be assigned to each region of the problem. Then the volume sources were added to the appropriate regions of the problem. Since most of the induced photon spectrum were composed of both discrete and continuum emissions, typically it was necessary to assign two volume sources to a region of the problem, one for the discrete source and one for the continuum source. All of the photon-only transport calculations in this work were run with $\mathrm{S}_{16}$ Triangular Chebychev Legendre angular quadrature set and a minimum of $\mathrm{P}_{2}$ Galerkin scattering moments. The same required checks for solution convergence were applied for the photon transport calculations.

The output parameters used for the photon transport calculation are different than those used for the neutron transport calculation. In order to compare the volumeaveraged photon flux to that obtained from MCNP, the energy-dependent volumeaveraged scalar flux edit was obtained. To obtain the required input for the detector 
response calculation, the multigroup angularly discretized photon flux at the detector location is calculated using the Attila LCF edit [11], an approach described more fully in [1] and [19]. The Triangular Chebychev Lobatto quadrature set with an $\mathrm{S}_{128}$ was used for the LCF calculation. The Triangular Chebychev Lobatto quadrature set was used because it has a quadrature point forwardly directed.

Although in a typical coupled neutron-photon calculation the photon flux within the detector volume would not be calculated, the comparison of the volume-averaged photon flux is more diagnostically relevant to this method development work than the detector response. Understanding if the proper induced photon spectrum was created within the moderating material and properly represented as a source term in Attila needed to be tested. The calculated photon production rate calculated within a volume could be compared to a reaction rate tally in MCNP if the photon yield for the interaction was unity, but this is not typically the case and could only be done for hydrogen, deuterium and carbon. This testing was completed through the comparison of the volume-averaged photon flux within the detector volume for all remaining benchmarking cases.

\subsubsection{Detector Response Calculation}

The final step was calculating the high-resolution gamma-ray spectrometer response for the prompt gamma neutron activation problem. The photon flux at the detector location obtained from Attila was used as the source definition for the detector model in MCNP. This energy-dependent angular flux was then used as the source term for a "near-field" Monte Carlo simulation for pulse-height tally in the gamma-ray spectrometer [1] [19]. This coupled deterministic-Monte Carlo approach combines the attractive advantages of deterministic transport for the radiation field calculations 
throughout a problem, with the inherent strength of Monte Carlo methods to calculate accurate pulse-height tallies for gamma-ray spectrometer modeling. The pulse-height tally was calculated using MCNP5 in this work, and recorded in uniform, narrow energy bins (typically 1-keV wide or less). The detector response reflects the angular fidelity of the Attila photon flux calculation as the coordinate system of the detector-scale Monte Carlo simulation was forced to be consistent with the coordinate system of the large-scale Attila calculation. While this method was utilized in this work, no modifications to the existing methods were necessary to facilitate the inclusion of this new class of problems. A detailed explanation of the mechanics and utility of the RADSAT coupling method is provided in [1] [19]. 


\section{CHAPTER 3}

\section{RESULTS}

Once the generally methodology had been developed, testing the methodology using code-to-code comparisons was an important part of the verification process. Completing the code-to-code comparison will indicate the capabilities and shortcomings of the method used in RADSAT to generate neutron-induced photons in material. This chapter contains the results of the code-to-code comparisons completed as part of the verification of the methodology using polyethylene, phosphorus, nitrogen, oxygen, and iron as materials of interest.

\subsection{Polyethylene}

Polyethylene was chosen as the first test case for the newly developed RADSAT neutron-gamma method for a number of reasons: the small number of neutron reactions which generate gamma rays, it is a molecule and not just an element, and the frequency that the material and/or its constituents would be exposed to neutrons. One frequent use for polyethylene is as a neutron shielding material and therefore often present around nuclear systems. Additionally, polyethylene and its constituents are present everywhere.

Many of the comparisons for polyethylene were included in Chapter 2 since it was used as the test case for decision making in the method development process. A comparison of the scalar neutron flux over all the entire neutron energy range and the thermal range within the polyethylene cube for Attila and MCNP are shown in Figure 9 and Figure 10, respectively. A comparison of the photon flux in the detector calculated using the volume-averaged neutron flux within the entire polyethylene cube with 
RADSAT, the average neutron flux within each of the subvolumes with RADSAT and a MCNP simulation is shown in Figure 14. The comparison of the RADSAT-NG and GROUPR cross-section libraries and the comparison of the MCNP and RADSAT detector responses are given in the following sections.

\subsubsection{Cross Sections}

Neutron interactions in polyethylene do not produce many gamma rays. None of its constituents produce any continuum gamma rays at all. Hydrogen has two naturally occurring isotopes, ${ }^{1} \mathrm{H}$ and ${ }^{2} \mathrm{H}$ with $99.9885 \%$ and $0.0115 \%$ natural abundance, respectively. Hydrogen and deuterium only produce one gamma ray each, both of which are from radiative capture. Carbon (elemental) produces four: three from radiative capture and one from inelastic scatter. The ${ }^{1} \mathrm{H},{ }^{2} \mathrm{H}$ and C RADSAT-NG cross sections were collapsed from the ENDF/B-VI cross section data at $300 \mathrm{~K}$. A comparison of the newly developed RADSAT-NG cross sections and the cross sections collapsed using GROUPR for the radiative capture cross sections for hydrogen, deuterium and carbon and the carbon inelastic scattering cross section are shown in Figure 17, Figure 18, Figure 19 and Figure 20, respectively. 


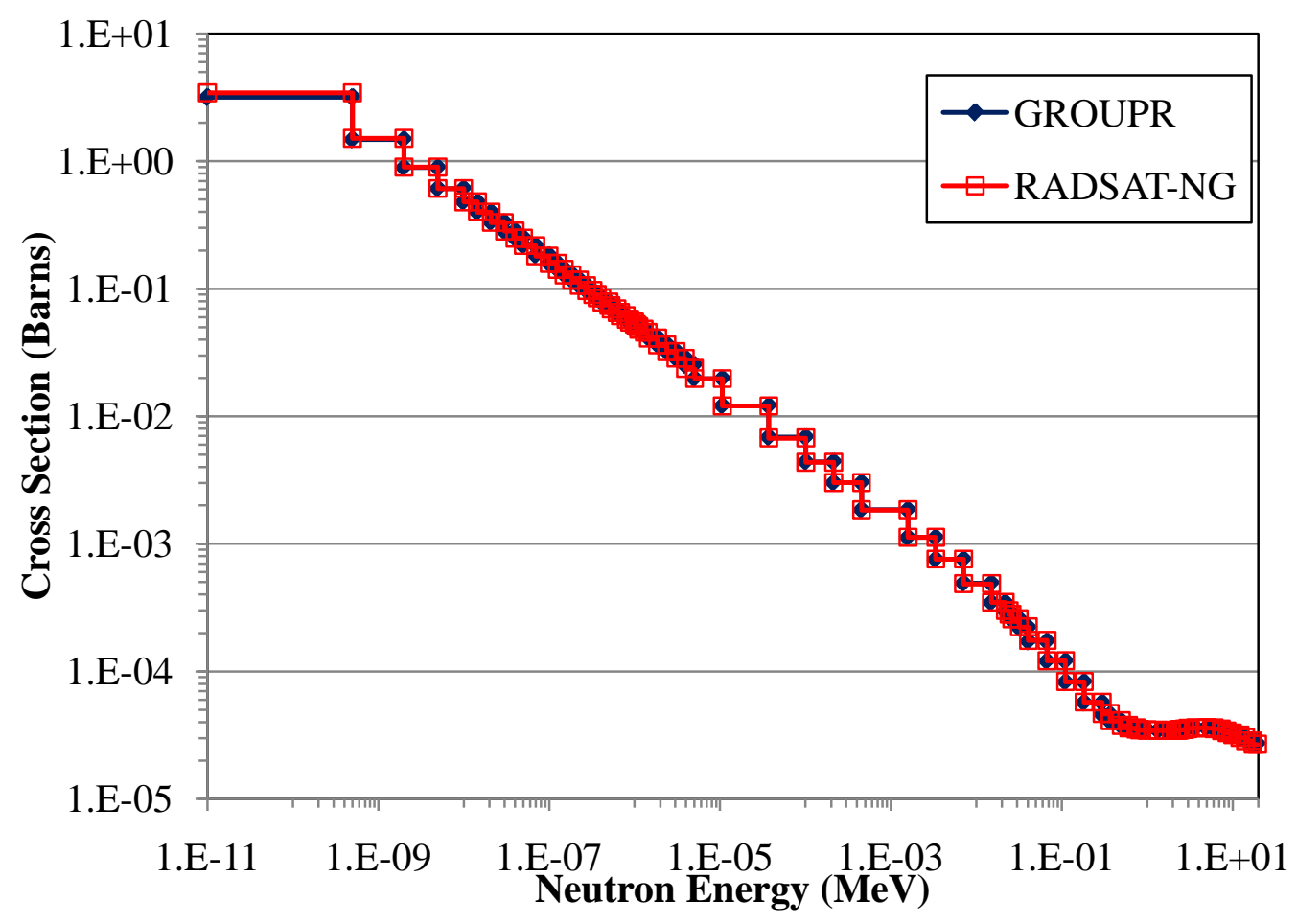

Figure 17. Comparison of Hydrogen Radiative Capture Photon Production Cross Section for RADSAT-NG and GROUPR

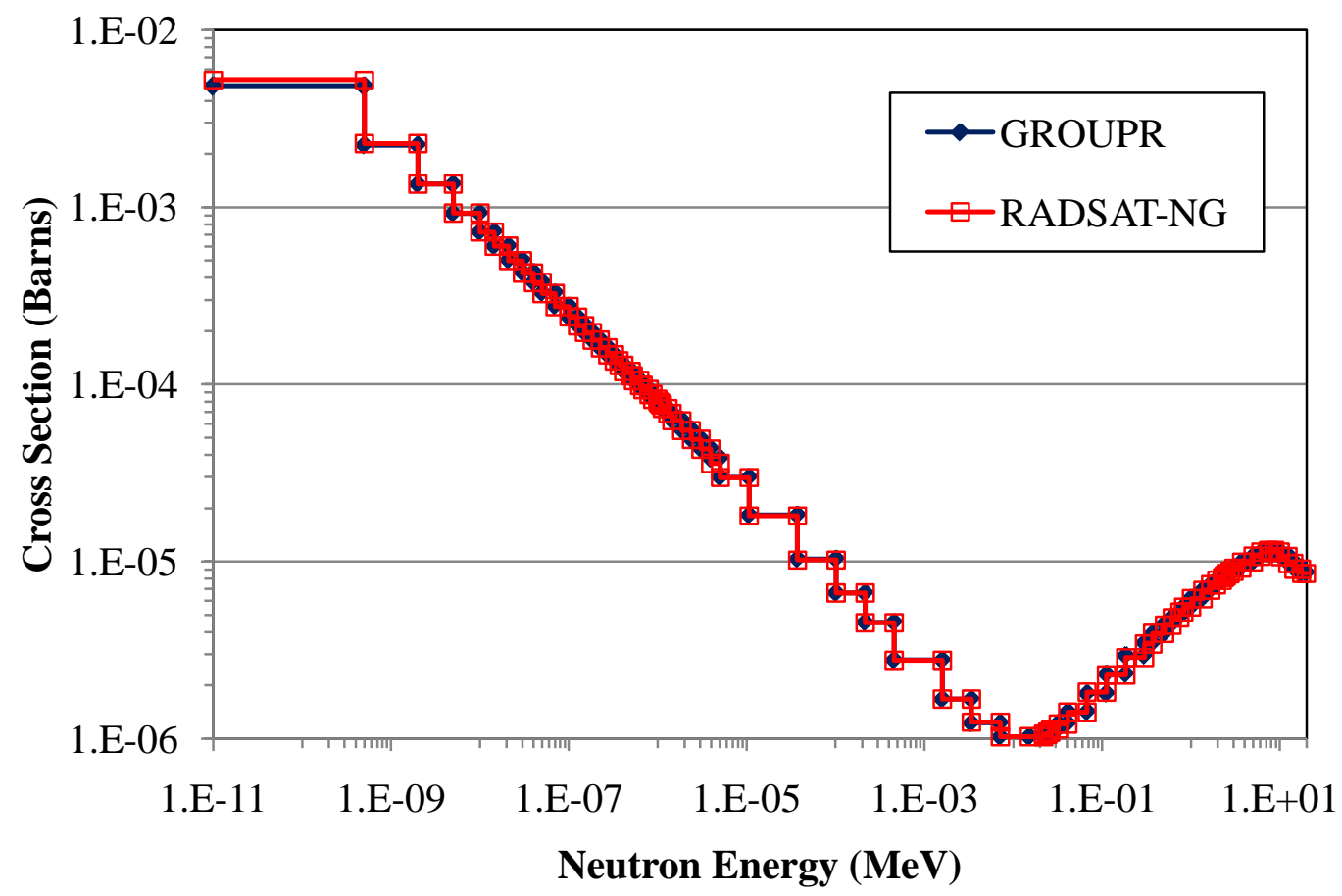

Figure 18. Comparison of Deuterium Radiative Capture Photon Production Cross Section for RADSAT-NG and GROUPR 


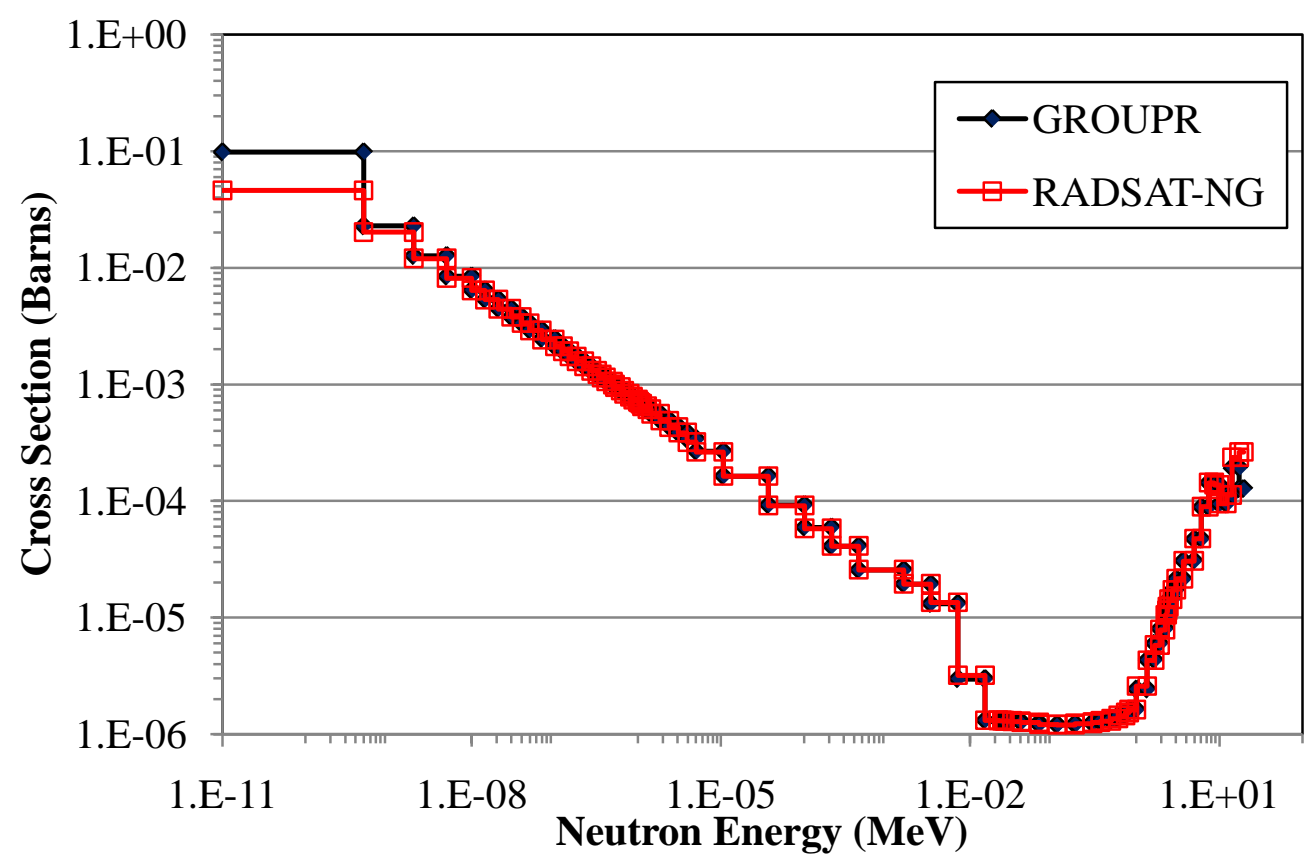

Figure 19. Comparison of Carbon Radiative Capture Photon Production Cross Section for RADSAT-NG and GROUPR

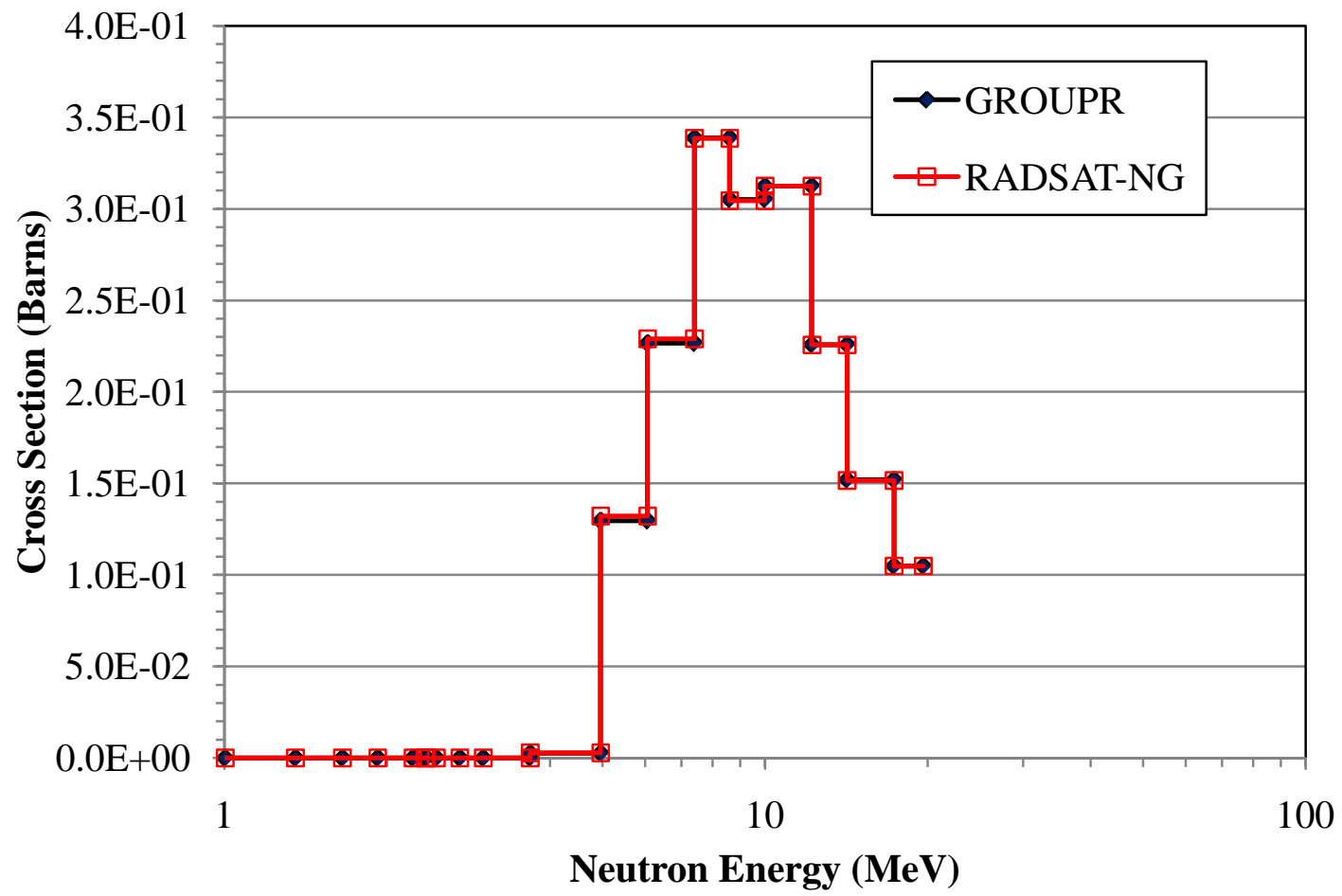

Figure 20. Comparison of Carbon Inelastic Scatter Photon Production Cross Section for RADSAT-NG and GROUPR 
The radiative capture cross sections for hydrogen, deuterium and carbon are all smoothly varying functions of neutron energy causing the collapsed cross sections to also be relatively smooth functions. A comparison of the total radiative capture cross section in hydrogen, deuterium and carbon for RADSAT-NG and collapsed using GROUPR are within $3 \%$. The inelastic scattering cross section for carbon is a threshold interaction, so the inelastic scatter photon production cross section in carbon is zero below $4.85-\mathrm{MeV}$. Unlike the radiative capture cross section, the inelastic scattering cross section for carbon does not smoothly vary with neutron energy as was shown previously in Figure 12. Although there is some variation in the collapsed results, the total carbon inelastic scattering cross section for RADSAT-NG and GROUPR differ by less than 3\%. Table 4 contains a comparison of the RADSAT-NG total cross section, total cross sections collapsed using GROUPR, the group containing the maximum variation, and the group containing the minimum variation for the hydrogen, deuterium and carbon radiative capture cross section and the carbon inelastic scatter cross section.

Table 4. Comparison of Total Cross Section for RADSAT-NG and Cross Sections Produced by GROUPR for Hydrogen, Deuterium and Carbon

\begin{tabular}{|l|c|c|c|c|c|}
\hline $\begin{array}{l}\text { Cross } \\
\text { Section }\end{array}$ & $\begin{array}{l}\text { RADSAT-NG } \\
\text { Total Cross } \\
\text { Section (Barns) }\end{array}$ & $\begin{array}{l}\text { GROUPR Total } \\
\text { Cross Section } \\
\text { (Barns) }\end{array}$ & $\begin{array}{l}\text { Percent } \\
\text { Difference }\end{array}$ & $\begin{array}{l}\text { Group \# } \\
\text { Largest \% } \\
\text { Difference }\end{array}$ & $\begin{array}{l}\text { Group \# } \\
\text { Smallest \% } \\
\text { Difference }\end{array}$ \\
\hline $\begin{array}{l}{ }^{1} \text { H Radiative } \\
\text { Capture }\end{array}$ & 10.424 & 10.12 & $3.02 \%$ & $\begin{array}{c}\text { Group \#79 } \\
(7.84 \%)\end{array}$ & $\begin{array}{l}\text { Group \#14 } \\
(1.78 E-3 \%)\end{array}$ \\
\hline $\begin{array}{l}{ }^{2} \text { H Radiative } \\
\text { Capture }\end{array}$ & 0.0161 & 0.0156 & $2.79 \%$ & $\begin{array}{c}\text { Group \#79 } \\
(8.51 \%)\end{array}$ & $\begin{array}{c}\text { Group \#14 } \\
(7.20 \mathrm{E}-3 \%)\end{array}$ \\
\hline $\begin{array}{l}\text { C Radiative } \\
\text { Capture }\end{array}$ & 0.141 & 0.137 & $1.56 \%$ & $\begin{array}{l}\text { Group \#1 } \\
(105.8 \%)\end{array}$ & $\begin{array}{c}\text { Group \#33 } \\
(4.68 \mathrm{E}-3 \%)\end{array}$ \\
\hline $\begin{array}{l}\text { C Inelastic } \\
\text { Scatter }\end{array}$ & 1.80 & 1.83 & $3.08 \%$ & $\begin{array}{c}\text { Group \#9 } \\
(37.4 \%)\end{array}$ & $\begin{array}{c}\text { Group \#6 } \\
(5.86 \mathrm{E}-1 \%)\end{array}$ \\
\hline
\end{tabular}


Most of the discrepancies between RADSAT-NG and GROUPR for hydrogen, deuterium and carbon were minor. For the carbon radiative capture photon production cross section, the RADSAT-NG cross section for energy group 1 appears to be approximately a factor of 2 greater than the GROUPR collapsed cross section. Also, the GROUPR set shows the cross section for group 1 lower than the cross section for group 2. Upon examining the pointwise cross section data obtained from ACER, which is shown graphically in Figure 11, it was noted that the cross section data were not decreasing in magnitude from the data within group 2. It was also noted that the largest percent difference for the RADSAT-NG and GROUPR radiative capture cross sections for hydrogen and deuterium were in group 79, and the largest percent difference for the carbon inelastic scatter reaction was in group 9, the first energy group containing a cross section. The discrepancies in the first and last energy group containing a cross section were investigated but no obvious data handling differences were found. The NJOY source code was examined to determine if there was a special treatment applied in this bin, but none could be found. It should be noted that although the comparison was made to GROUPR, it was determined that exactly replicating the data produced by GROUPR was not necessary. The differences were noted and the cross section set was tested through benchmarking against MCNP problem.

\subsubsection{Detector Response}

In order to show how the photon angular flux at the detector location translates to the detector response, the calculation of the detector response using the RADSAT method was completed. As stated in Section 2.2.4, the detector response calculation is part of the existing RADSAT package and was only drawn upon for these simulations. The 
RADSAT method entailed a near field MCNP calculation containing only the detector using the photon angular flux calculated in Attila as the source term. The photon angular flux was calculated using the spatially dependent neutron-induced photon spectrum as the source term for the photon transport calculation as indicated in Section 2.2.1.2. This was then compared to a full scale MCNP calculation which included the full geometry for the neutron activation analysis of polyethylene and the results are shown in Figure 21. The same detector geometry was specified in both the RADSAT and MCNP simulations as outlined in Section 2.1, and the same Gaussian energy broadening (or GEB) was applied to both spectra. The GEB in MCNP is a parameter that allows one to "specify the full width at half maximum of the observed energy broadening in a physical radiation detector" [2].

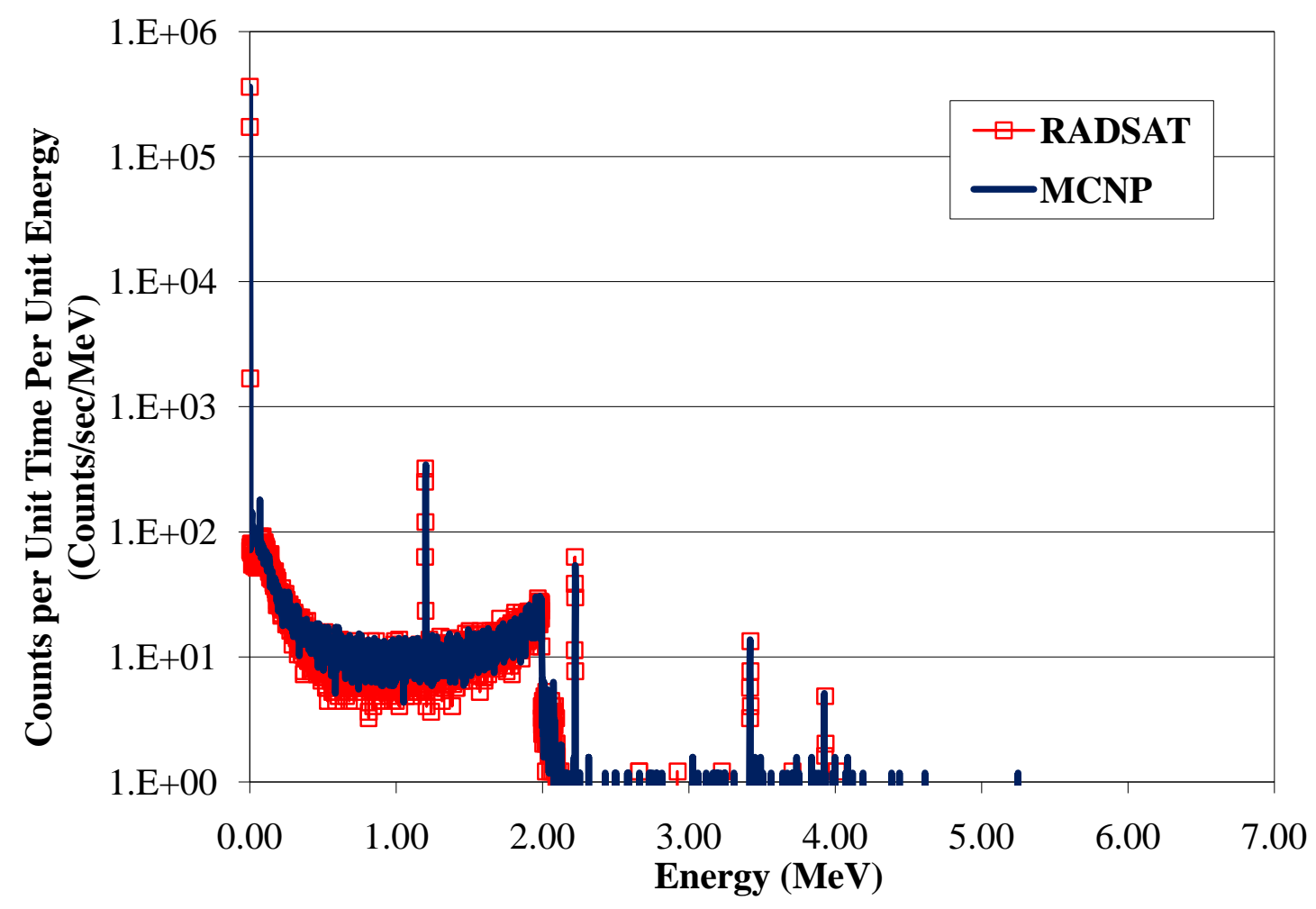

Figure 21. RADSAT and MCNP Detector Response for Neutron Irradiated Polyethylene 
The total count rate in the prominent peak regions agreed to within $5 \%$ for the RADSAT and MCNP simulations. The statistical uncertainty of the MCNP simulation varied from $3.9 \%$ in the region of the $1.2-\mathrm{MeV}$ line to $27.7 \%$ in the region of the 3.9 MeV line. The large statistical uncertainty associated with the MCNP calculation is due to the short count time (600 second). Additionally, the double-escape peak at $1.20-\mathrm{MeV}$ is enhanced due to the small detector size.

\subsection{Phosphorus}

Phosphorus was chosen as the next benchmarking experiment to study an increased complexity in the cross sections. Phosphorus is commonly found in fertilizer, pesticides, explosives, fireworks, nerve agents, detergents and toothpaste. Knowing the neutron-induced photon spectrum from phosphorus could allow for the identification of explosives or nerve agents using active interrogation methods.

\subsubsection{Neutron Flux Comparison}

Although blocks of solid phosphorus are not found in nature due to its high chemical reactivity, a solid block of pure phosphorus was modeled to test the accuracy of the RADSAT-NG cross section. The density of the phosphorus was modeled at 1.82 grams $/ \mathrm{cm}^{3}$, the natural density of phosphorus [31]. Unlike in the polyethylene simulation, the phosphorus cube does not shield the germanium detector from fast neutrons. Since the purpose of these benchmarking cases was not to simulate the activation of the germanium detector, the neutron importance within the detector in the MCNP simulation was set to a very small number, $1 \times 10^{-10}$, reducing the number of neutron interactions that will occur within the volume of the germanium detector. This 
will reduce the effect of the detector on the volume-averaged photon flux within the detector volume. By reducing the neutron importance in the detector material, the backscatter from the detector into the phosphorus cube is also reduced. Additionally, MCNP will not allow importance weighting in problems where pulse-height tallies are calculated. Although the comparison to the detector response is important, the method of calculating the detector response from the photon angular flux in Attila was unaltered as part of the method development in this work. This method has been well tested and proven to work in previous RADSAT publications [1][19]. Additionally, the detector response comparison has been made for the polyethylene case in this work. Since it is more diagnostically relevant for testing this new method to compare only the activation of the material of interest for a given benchmark problem, the importance weighting was used for phosphorus and all the other materials except polyethylene. A comparison of the volume-averaged neutron flux within the phosphorus cube for MCNP and Attila is shown in Figure 22. 


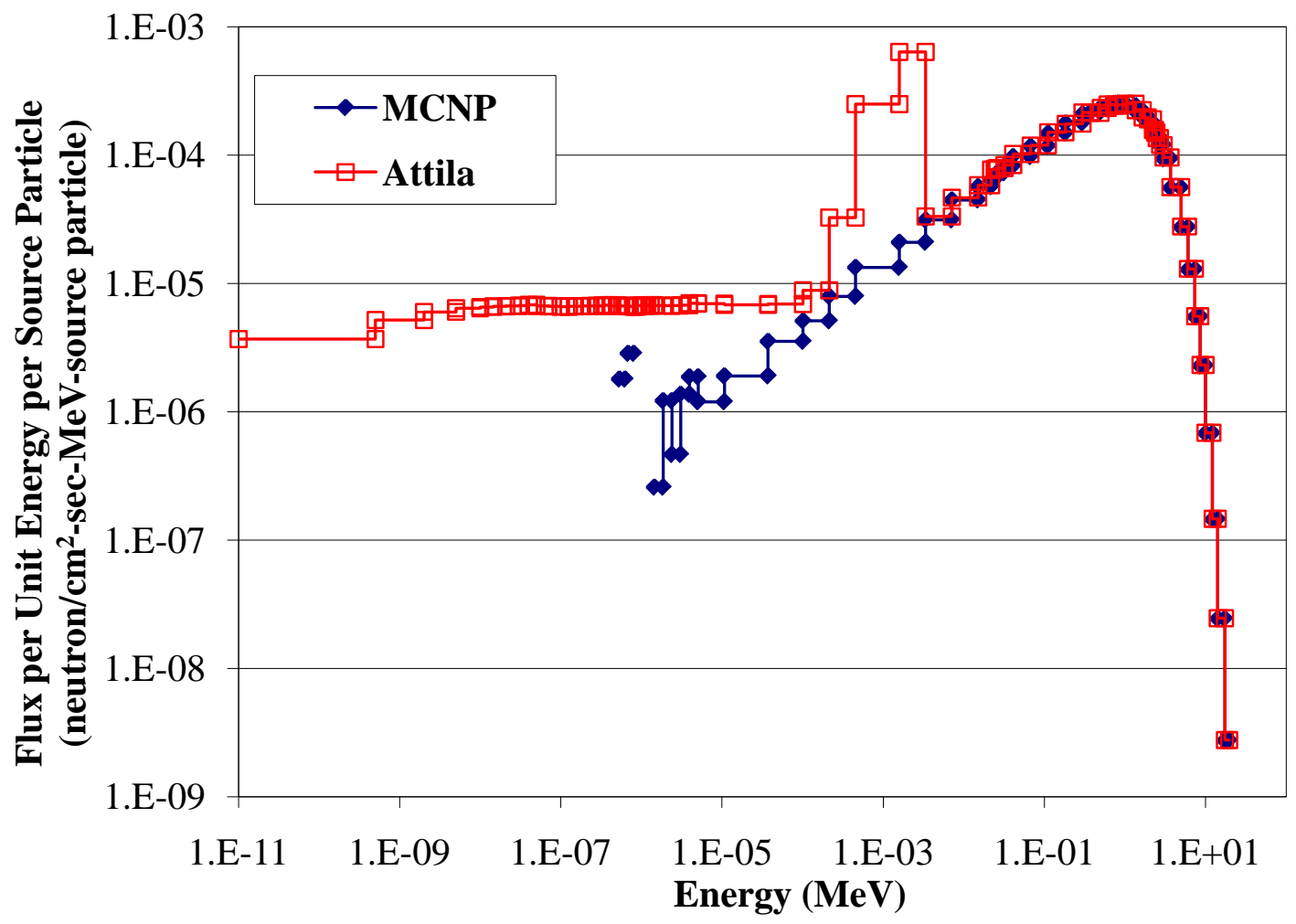

Figure 22. Comparison of Attila and MCNP Neutron Flux for the Irradiation of Phosphorus

The Attila result is able to resolve the entire solution, whereas MCNP does not obtain a result in every energy bin. The statistical uncertainty for the MCNP calculation from $13.7-\mathrm{eV}$ to $10.7-\mathrm{eV}$ is $9.04 \%$, and the statistics below $5-\mathrm{eV}$ are above $42.82 \%$. There is a bias in the Attila neutron flux results which is seen in all of the remaining neutron transport cases below approximately $50-\mathrm{eV}$. It is believed that this bias is due to the small number of neutrons which actually scatter into this region. Additionally, the discrepancy in the neutron flux from Attila in the energy range of $0.214-\mathrm{keV}$ to $3.35-\mathrm{keV}$ appears to be an artifact of the Kynea3 cross-section library. Analysis of the original ENDF/B-VI data, the data from which the Kynea3 cross section library was derived, does not indicate the presence of a multiplicity interaction $\left(n,{ }^{*} n\right)$ that would account for the 
increase in neutrons in this energy range. Tests were also completed with MCNP using ENDF/B-VI.0 cross section instead of the ENDF/B-VI.6 cross section for phosphorus to determine if the shape was an artifact of the cross section library, but the shape could not be reproduced. Comparisons can be made using more realistic materials and experimental setups to test the biasing in the thermal region, so the computational models can be compared to experiment to determine which method is more accurately depicting the neutron spectrum. Additional comparisons of the neutron transport methods were outside the scope of this body of work. The total neutron flux for the Attila calculation was within $1.07 \%$ of the neutron flux calculated by the MCNP simulation. A comparison of the total neutron flux is shown in Table 5.

Table 5. Comparison of Attila and MCNP Total Neutron Flux for the Irradiation of Phosphorus

\begin{tabular}{|l|l|l|l|}
\hline $\begin{array}{l}\text { MCNP Flux } \\
\text { (Neutron } / \mathbf{c m}^{2} \text {-sec- } \\
\text { source particle) }\end{array}$ & $\begin{array}{l}\text { MCNP Statistical } \\
\text { Uncertainty }\end{array}$ & $\begin{array}{l}\text { Attila Flux } \\
\text { (Neutron } / \mathbf{c m}^{2} \text {-sec- } \\
\text { source particle) }\end{array}$ & $\begin{array}{l}\text { Percent } \\
\text { Difference }\end{array}$ \\
\hline $7.67 \times 10-4$ & $0.01 \%$ & $7.75 \times 10-4$ & $1.07 \%$ \\
\hline
\end{tabular}

\subsubsection{Cross Sections}

Like polyethylene, phosphorus does not have a complex induced gamma-ray

spectrum. Within the Kynea3 energy range, the cross section data for ${ }^{31} \mathrm{P}$ designates that two continua are produced, one by radiative capture and one by an unidentified interaction, in addition to two discrete gamma rays produced by an unidentified reaction. In the ENDF format, photons produced by unidentified reactions are designated as originating from "nonelastic interactions." Table 6 contains a list of the gamma-ray 
producing interactions in ${ }^{31} \mathrm{P}$ and the discrete gamma rays produced by each interaction. All of the photon production cross sections for phosphorus are smoothly varying functions of energy containing no resonances. The ${ }^{31} \mathrm{P}$ RADSAT-NG cross sections were collapsed from the ENDF/B-VI cross section data at 300K. A graphical comparison of the RADSAT-NG and GROUPR radiative capture cross section and "nonelastic" cross sections for phosphorus are shown in Figure 23 and Figure 24.

Table 6. Discrete Gamma Rays Produced by ${ }^{31} \mathrm{P}$

\begin{tabular}{|l|l|}
\hline Reaction & Gamma Rays $(\mathrm{MeV})$ \\
\hline Nonelastic & $1.266,2.234$ \\
\hline
\end{tabular}

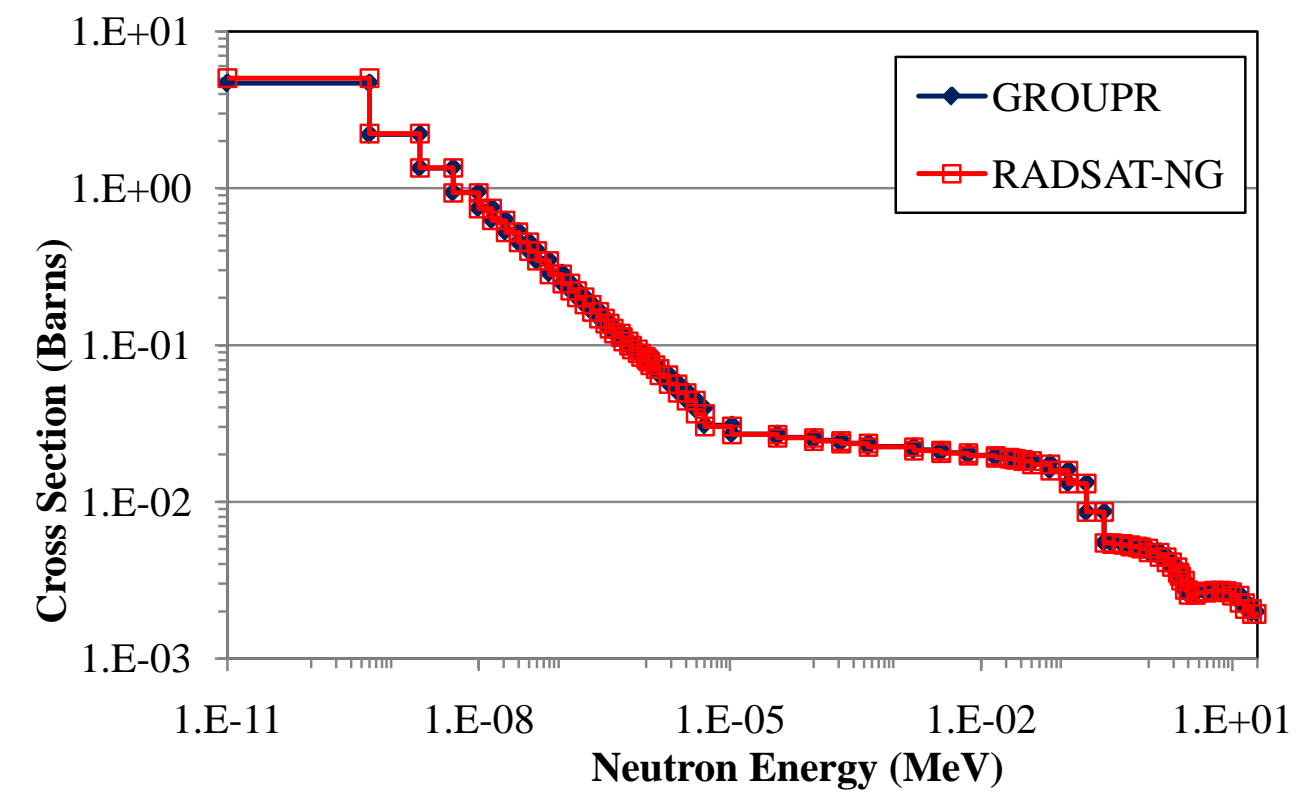

Figure 23. Comparison of Phosphorus Radiative Capture Photon Production Cross Section for RADSAT-NG and GROUPR

The comparison of the RADSAT-NG and GROUPR collapsed cross sections show good agreement for phosphorus. The total RADSAT-NG radiative capture and 
"nonelastic" cross sections are within $0.1 \%$ and $1.9 \%$ of the GROUPR collapsed cross sections, respectively. The energy group with the largest percent difference for the radiative capture cross section was group number 79 at $7.5 \%$. Energy group 19, the first energy group containing a cross section for the "nonelastic interaction" in phosphorus, has a difference of $7.84 \%$. A comparison of the RADSAT-NG total cross section, total cross sections collapsed using GROUPR, the group containing the maximum variation, and the group containing the minimum variation is shown in Table 7.

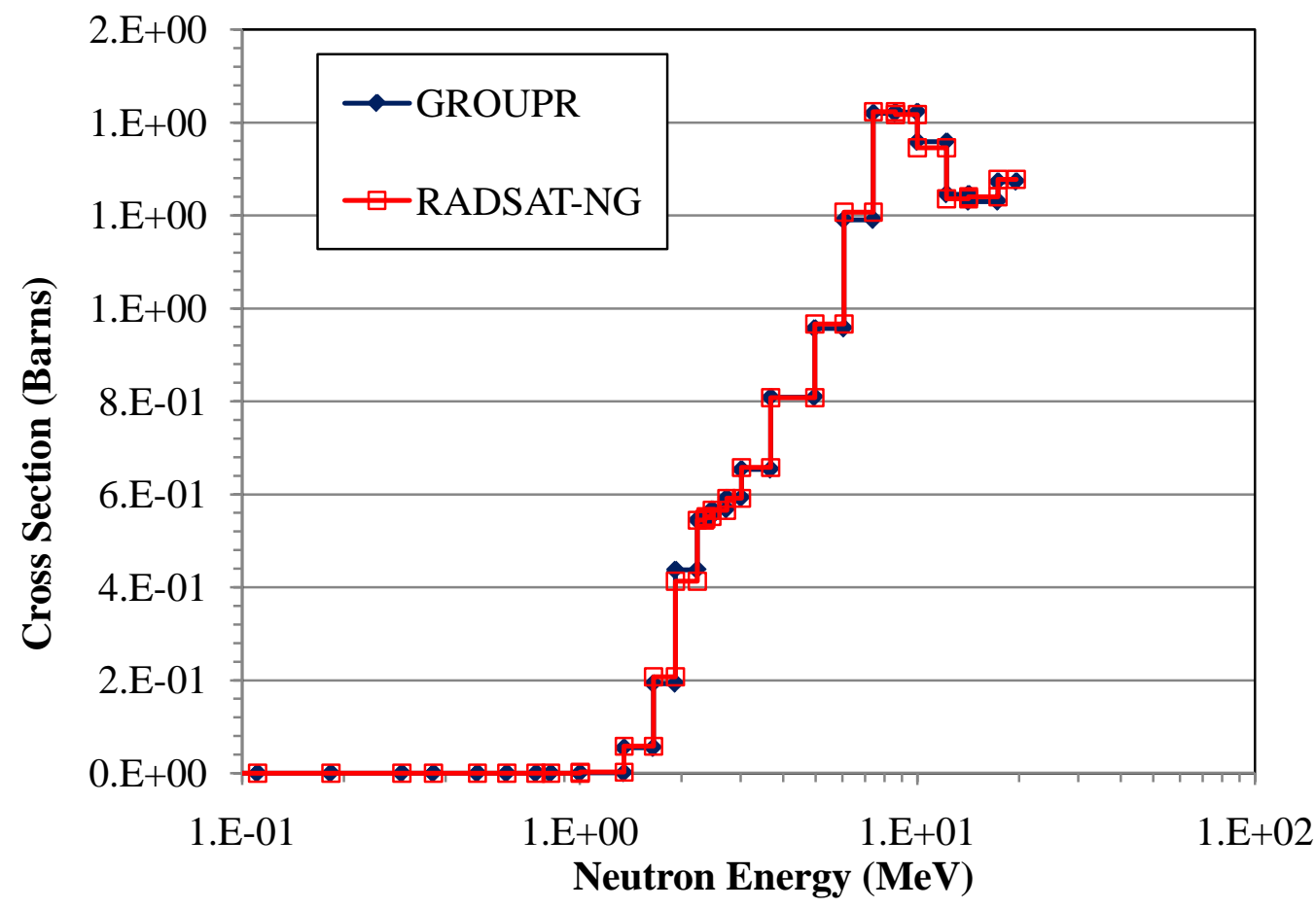

Figure 24. Comparison of Phosphorus "Nonelastic" Photon Production Cross Section for RADSAT-NG and GROUPR 
Table 7. Comparison of Total Cross Section for RADSAT-NG and Cross Sections Produced by GROUPR for Phosphorus

\begin{tabular}{|l|l|l|l|l|l|}
\hline $\begin{array}{l}\text { Cross } \\
\text { Section }\end{array}$ & $\begin{array}{l}\text { RADSAT-NG } \\
\text { Total Cross } \\
\text { Section (Barns) }\end{array}$ & $\begin{array}{l}\text { GROUPR Total } \\
\text { Cross Section } \\
\text { (Barns) }\end{array}$ & $\begin{array}{l}\text { Percent } \\
\text { Difference }\end{array}$ & $\begin{array}{l}\text { Group \# } \\
\text { Largest \% } \\
\text { Difference }\end{array}$ & $\begin{array}{l}\text { Group \# } \\
\text { Smallest \% } \\
\text { Difference }\end{array}$ \\
\hline $\begin{array}{l}\text { Nonelastic } \\
\text { Interaction }\end{array}$ & 15.06 & 15.05 & $0.10 \%$ & $\begin{array}{l}\text { Group \#19 } \\
(7.84 \%)\end{array}$ & $\begin{array}{l}\text { Group \#14 } \\
(5.52 \mathrm{E}-2 \%)\end{array}$ \\
\hline $\begin{array}{l}\text { Radiative } \\
\text { Capture }\end{array}$ & 16.14 & 15.84 & $1.87 \%$ & $\begin{array}{l}\text { Group \#79 } \\
(7.51 \%)\end{array}$ & $\begin{array}{l}\text { Group \#77 } \\
(2.16 \mathrm{E}-2 \%)\end{array}$ \\
\hline
\end{tabular}

\subsubsection{Photon Flux within Detector}

Using the RADSAT-NG cross sections and the spatially dependent neutron flux obtained from Attila, the neutron-induced photon spectrum was calculated for the irradiation of phosphorus. Using the group generator in RADSAT, 150 continuum groups and three $0.3-\mathrm{keV}$ wide peak groups were assigned, one for the $511-\mathrm{keV}$ annihilation peak and the other two for the two discrete gamma rays produced by phosphorus. A $1 / \sqrt{E}$ weighting factor was applied to the continuum groups which makes the continuum groups in the lower energy range finer and the continuum groups in the higher energy range broader. This group structure was used to create the photon cross-section library for the photon transport calculation.

A comparison of the volume-averaged gamma flux in the detector for MCNP and RADSAT for the irradiation of phosphorus is shown in Figure 25. The comparison of MCNP and RADSAT shows good agreement for both the continuum and the peaks. The total flux simulated using MCNP and RADSAT shows agreement to within $0.13 \%$, and the statistical uncertainty of the MCNP simulation was $0.92 \%$. A comparison of the 1.266 and 2.234-MeV gamma rays for MCNP and RADSAT shows typical agreement to 
within 0.42 and $1.23 \%$, respectively. The statistical uncertainty of the MCNP calculations was 1.43 and $2.31 \%$ for those same lines and is shown in Table 8 .

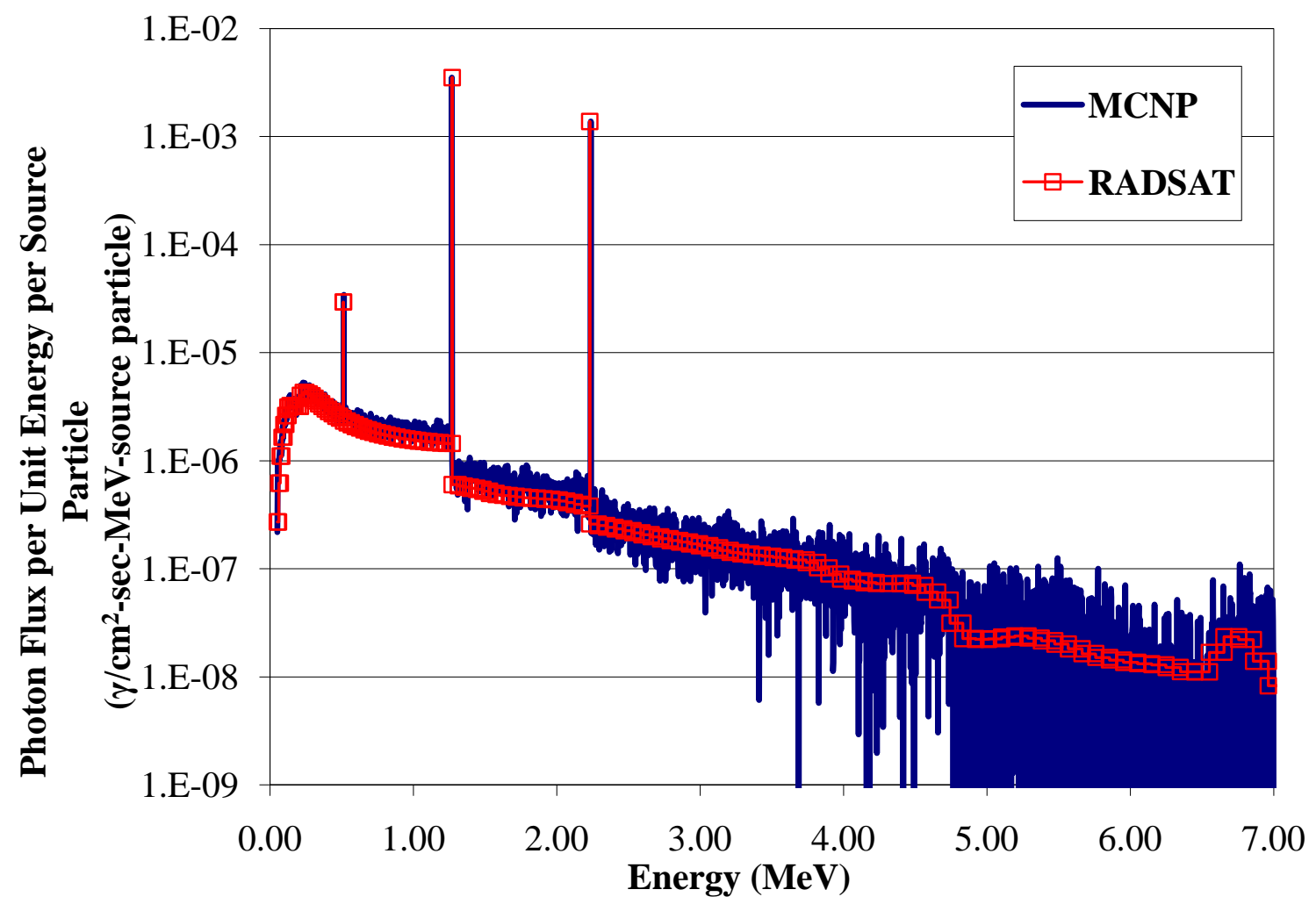

Figure 25. Code-to-Code Comparison Using RADSAT and MCNP5 of VolumeAveraged Flux at the Detector Location for Neutron Activation Analysis of Phosphorus

Table 8. Comparison of Photon Flux for Top 2 Most Intense Peaks Produced by the Neutron Irradiation of Phosphorus

\begin{tabular}{|l|l|l|l|l|}
\hline $\begin{array}{l}\text { Gamma-Ray } \\
\text { Energy (MeV) }\end{array}$ & $\begin{array}{l}\text { MCNP } \\
\text { ( } / \text { /sec-Source } \\
\text { Particle) }\end{array}$ & $\begin{array}{l}\text { MCNP Relative } \\
\text { Uncertainty } \\
(\%)\end{array}$ & $\begin{array}{l}\text { RADSAT } \\
\text { ( } / \text { /sec-Source } \\
\text { Particle) }\end{array}$ & $\begin{array}{l}\text { Percent } \\
\text { Difference } \\
(\%)\end{array}$ \\
\hline 1.266 & $3.53 \mathrm{E}-06$ & 1.43 & $3.52 \mathrm{E}-06$ & 0.42 \\
\hline 2.234 & $1.37 \mathrm{E}-06$ & 2.31 & $1.38 \mathrm{E}-06$ & 1.23 \\
\hline Total & $8.48 \mathrm{E}-06$ & 0.92 & $8.47 \mathrm{E}-06$ & 0.13 \\
\hline
\end{tabular}




\subsection{Nitrogen}

Nitrogen was chosen as a testing material due to its common presence in nature and its relatively complex induced photon spectrum when compared to either polyethylene or phosphorus. Nitrogen is one of the most abundant elements on earth. It is present in the air, the earth's crust and many man-made substances including fertilizer, ammonia, nitric acid, propellants (rocket fuel) and explosives. The abundance of nitrogen in the atmosphere and the ground, in addition to being a constituent of explosives, makes it a key element for a coupled neutron photon simulations.

\subsubsection{Neutron Flux Comparison}

The neutron flux comparison was completed to test the accuracy of the Kynea3 cross section for nitrogen. Since pure nitrogen is typically only present in low densities (natural density of $0.001251 \mathrm{grams} / \mathrm{cm}^{3}$ at $0^{\circ} \mathrm{C}$ and $0.807 \mathrm{grams} / \mathrm{cm}^{3}$ at $-196^{\circ} \mathrm{C}$ for liquid nitrogen), an arbitrary density 0.5 grams per cubic centimeter was modeled to increase the probability of the neutron interaction in the nitrogen. The total neutron flux within the nitrogen cube for Attila is within $1.25 \%$ of the MCNP simulation result and is shown in Table 9. The Attila result was able to resolve the flux at the lower energy ranges causing the total flux to be higher. The MCNP simulation did not obtain a neutron flux in many of the lower energy bins. Although the MCNP statistical uncertainty for the tally is less than $1 \times 10^{-4} \%$, the statistical uncertainty for the $10-\mathrm{eV}$ energy bin is $11.19 \%$, and the statistical uncertainty for the 5-eV energy bin and below is greater than or equal to 20.21\%. A graphical comparison of the volume-averaged neutron flux within the nitrogen cube for MCNP and Attila is shown in Figure 26. Below approximately 50-keV, the Attila neutron flux appears to be biased. It is hypothesized that this is due to the 
small number of thermal neutrons created in this problem. In order for either code to converge of the proper answer, a problem should be set up which allows for the generation of more thermal neutrons.

Table 9. Comparison of Attila and MCNP Total Neutron Flux for the Irradiation of Nitrogen

\begin{tabular}{|l|l|l|l|}
\hline $\begin{array}{l}\text { MCNP Flux } \\
\text { (Neutron } / \mathbf{c m}^{2} \text {-sec- } \\
\text { source particle) }\end{array}$ & $\begin{array}{l}\text { MCNP Statistical } \\
\text { Uncertainty }\end{array}$ & $\begin{array}{l}\text { Attila Flux } \\
\text { (Neutron } / \mathbf{c m}^{2} \text {-sec- } \\
\text { source particle) }\end{array}$ & $\begin{array}{l}\text { Percent } \\
\text { Difference }\end{array}$ \\
\hline $7.67 \times 10^{-4}$ & $<1 \times 10^{-4} \%$ & $7.77 \times 10^{-4}$ & $1.25 \%$ \\
\hline
\end{tabular}

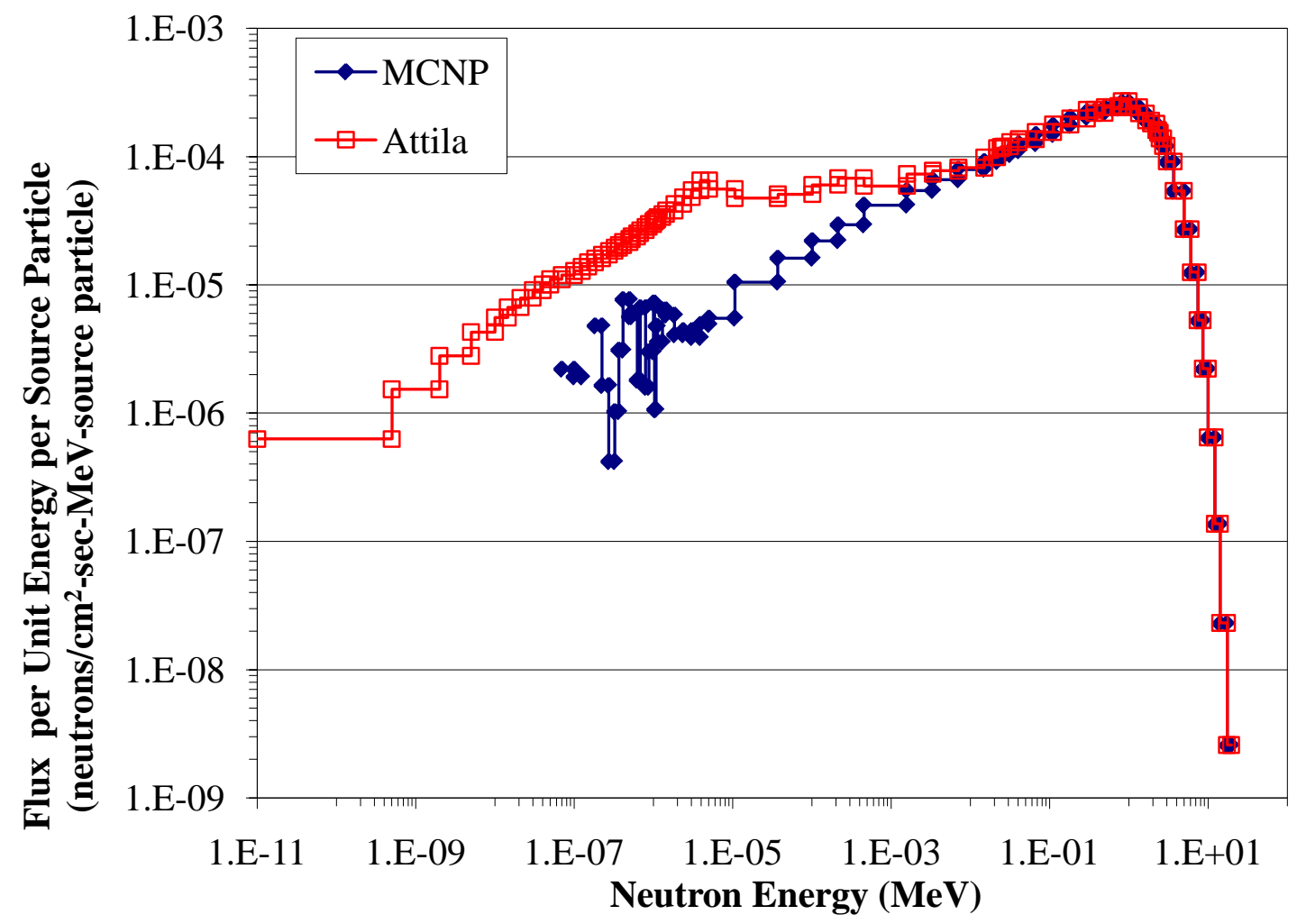

Figure 26. Comparison of Attila and MCNP Neutron Flux for the Irradiation of Nitrogen 


\subsubsection{Cross Sections}

The multigroup cross section for nitrogen is somewhat more difficult to calculate than the phosphorus cross section data. Nitrogen has two naturally occurring isotopes ${ }^{14} \mathrm{~N}$ and ${ }^{15} \mathrm{~N}$, with corresponding natural abundances of $99.634 \%$ and $0.366 \% .{ }^{14} \mathrm{~N}$ has eight gamma-ray producing reactions and ${ }^{15} \mathrm{~N}$ has eight gamma-ray producing reactions in the neutron energy range included in the Kynea3 neutron energy group structure, none of which contain any unresolved resonances. ${ }^{15} \mathrm{~N}$ produces eight continua and no discrete gamma rays, and there are a total of 136 discrete gamma-rays and one continuum produced by ${ }^{14} \mathrm{~N}$. Table 10 contains a list of the gamma producing interactions in ${ }^{14} \mathrm{~N}$ and the gamma rays produced by each interaction. The ${ }^{14} \mathrm{~N}$ continuum gamma rays are produced by the radiative capture cross section. The ${ }^{14} \mathrm{~N}$ and ${ }^{15} \mathrm{~N}$ RADSAT-NG cross sections were collapsed from the ENDF/B-VI cross section data at $300 \mathrm{~K}$. A comparison of the RADSAT-NG and GROUPR radiative capture cross section for ${ }^{14} \mathrm{~N}$ and the (n,np) reaction in ${ }^{15} \mathrm{~N}$ is shown in Figure 27 and Figure 28, respectively. 
Table 10. Discrete Gamma Rays Produced by ${ }^{14} \mathrm{~N}$

\begin{tabular}{|l|l|}
\hline Reaction & Gamma Rays $(\mathrm{MeV})$ \\
\hline$(\mathrm{n}, \mathrm{n})$ & $0.6119201,0.7283601,0.9670002,1.15779,1.34028,1.635302,2.14313$, \\
& $2.312799,2.498071,2.517631,2.655651,2.682731,2.726081,2.793093$, \\
& $2.928261,3.081021,3.294651,3.338001,3.378643,3.384011,3.480811$, \\
& $3.890703,3.948101,4.018801,4.365831,4.671601,4.716323,4.915101$, \\
& $5.105891,5.691441,5.834251,6.203501,6.446171,6.859454,7.029122$, \\
& $7.966902,8.062002,8.619702,8.776002,9.128902,9.172252,10.432,11.05$ \\
\hline$(\mathrm{n}, \mathrm{np})$ & $0.1693,0.5951001,0.7644001,3.089401,3.684501,3.853801$ \\
\hline$(\mathrm{n}, \mathrm{nd})$ & 4.438911 \\
\hline$(\mathrm{n}, \gamma)$ & $0.1314,0.383,0.5838,0.6083,0.7678,0.7704,0.8312,0.9084,1.0117$, \\
& $1.0252,1.0539,1.073,1.6108,1.6783,1.6812,1.7836,1.854,1.8848,1.9885$, \\
& $1.9997,2.0023,2.0308,2.2474,2.2618,2.2932,2.5204,2.726,2.8308$, \\
& $2.8984,3.0136,3.2692,3.3007,3.532,3.6777,3.8556,3.8809,3.8842$, \\
& $3.9239,4.5087,4.6541,5.2692,5.2978,5.5334,5.5621,6.3224,7.1534$, \\
& $7.299,8.3102,8.5686,9.0467,9.149,9.1519,9.2195,9.7571,9.9213$, \\
& $10.0619,10.6978,10.83291$ \\
\hline$(\mathrm{n}, \mathrm{p})$ & $0.4956001,0.6132001,0.6344001,0.8088001,0.9182001,1.2476,6.093801$, \\
& $6.589401,6.728202,7.012002,7.341402$ \\
\hline$(\mathrm{n}, \mathrm{d})$ & $0.1693,0.5951001,0.7644001,3.089401,3.684501,3.853801$ \\
\hline$(\mathrm{n}, \mathrm{t})$ & 4.438911 \\
\hline$(\mathrm{n}, \alpha)$ & $1.77149,2.12469,2.265201,2.298011,2.442101,2.840621,2.895621$, \\
& $3.539991,4.115411,4.444891,4.475311,4.667111,4.740111,5.020311$, \\
& $5.853151,6.435611,6.742902,6.791802,7.285512,7.977842,8.560302$, \\
& $8.920202,9.185002$ \\
\hline
\end{tabular}

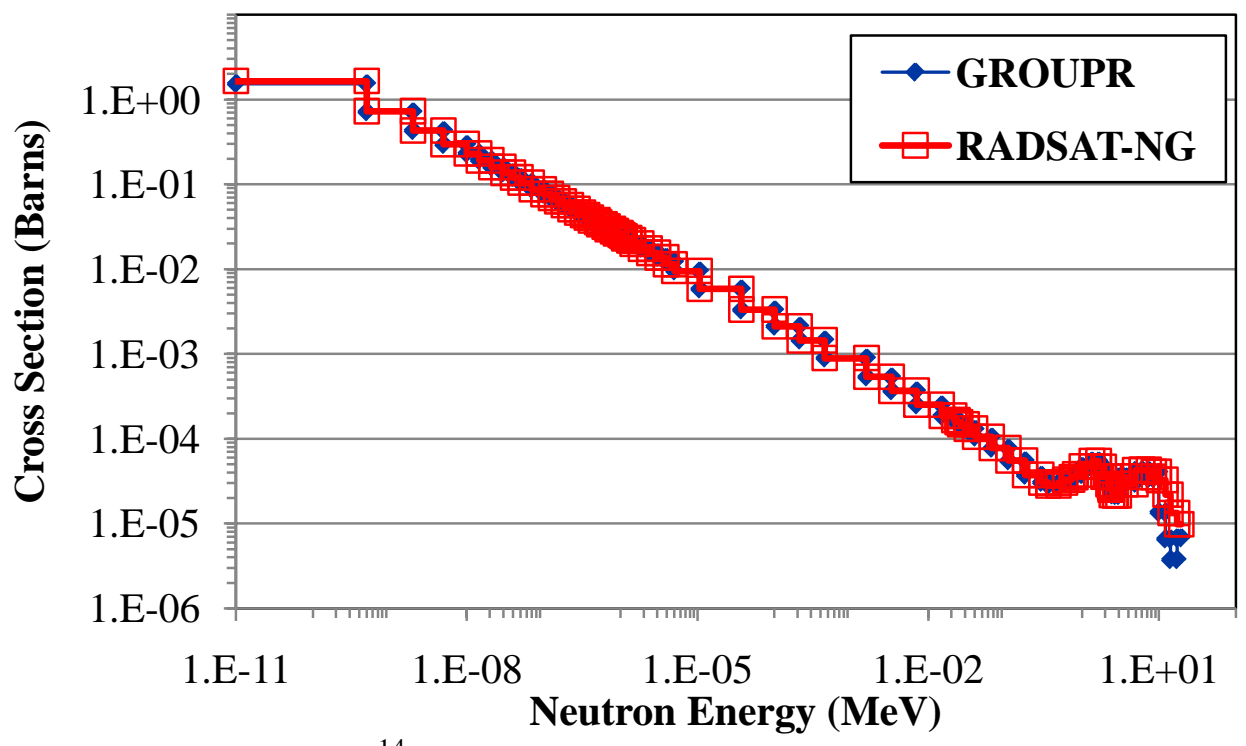

Figure 27. Comparison of ${ }^{14} \mathrm{~N}$ Radiative Capture Photon Production Cross Section for RADSAT-NG and GROUPR 


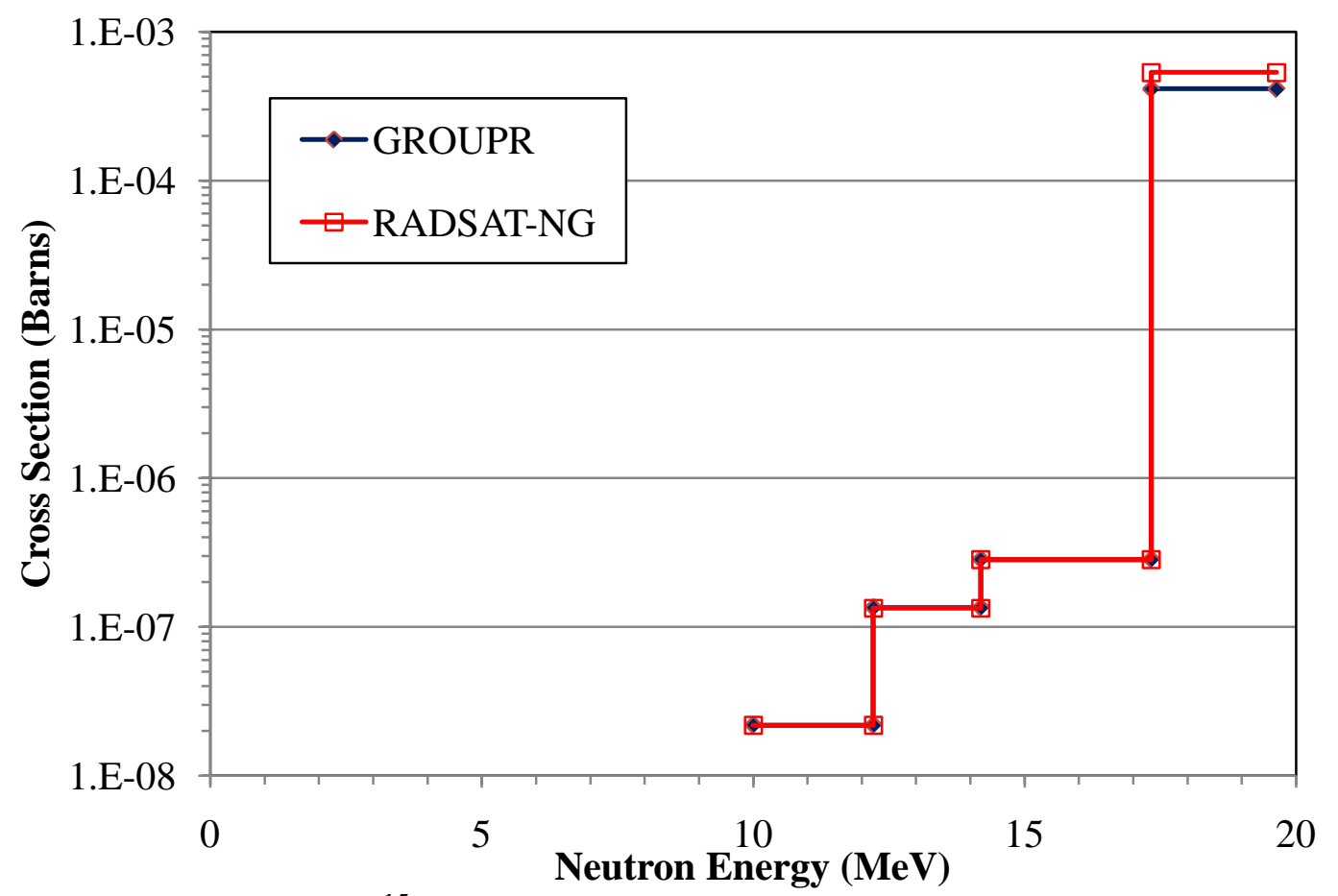

Figure 28. Comparison of ${ }^{15} \mathrm{~N}$ (n,np) Photon Production Cross Section for RADSAT-NG and GROUPR

The nitrogen RADSAT-NG and GROUPR collapsed nitrogen cross sections agree reasonably well. The comparison of the total RADSAT-NG ${ }^{15} \mathrm{~N}(\mathrm{n}, \mathrm{np})$ cross section is within $28.82 \%$ of the GROUPR collapsed cross section. The large percent difference for this cross section is due to the difference in the cross section in highest energy group, $5.39 \times 10^{-4}$ for RADSAT-NG and $4.13 \times 10^{-4}$ for GROUPR which accounts for $99.893 \%$ of the total cross section. For the ${ }^{14} \mathrm{~N}$ radiative capture cross section, there is a discrepancy in the cross section between the four highest energy groups when comparing the RADSAT-NG cross to the GROUPR collapsed cross section. The energy-dependent RADSAT-NG radiative capture cross section for ${ }^{14} \mathrm{~N}$ is compared to the GROUPR collapsed cross section and the energy-dependent ACER cross section for the same interaction in Figure 29 to examine this discrepancy against the energy-dependent cross 
section data. For the region between 10 and 19.6-MeV (neutron energy groups 1 thru 4), it appears that the RADSAT-NG cross section more accurately represents the pointwise data. This discrepancy is the main source of the $2.62 \%$ difference in the ${ }^{14} \mathrm{~N}$ radiative capture cross section when comparing the cross section produced by GROUPR and RADSAT-NG.

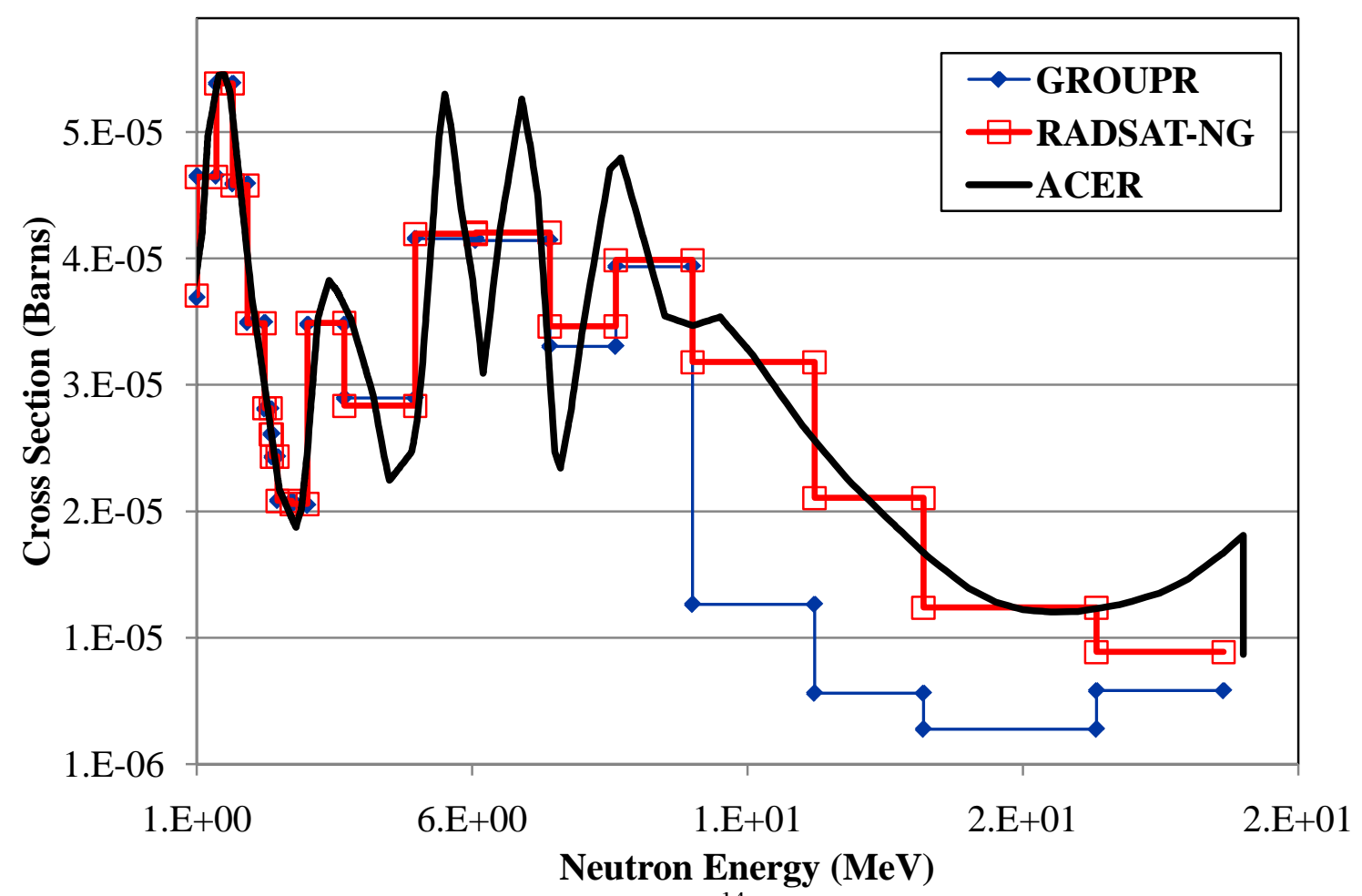

Figure 29. Comparison of Energy-dependent ${ }^{14} \mathrm{~N}$ Radiative Capture Photon Production Cross Section for RADSAT-NG, GROUPR and ACER

Table 11 contains a comparison of the total for each of the gamma-ray producing cross sections for ${ }^{14} \mathrm{~N}$ and ${ }^{15} \mathrm{~N}$ for RADSAT-NG and GROUPR and the percent difference for the two. All of the ${ }^{14} \mathrm{~N}$ RADSAT-NG cross sections are within $1.5 \%$ of the GROUPR cross sections except for the radiative capture cross section. The comparison 
of the ${ }^{15} \mathrm{~N}$ RADSAT-NG cross sections to the GROUPR cross section was not as close as the ${ }^{14} \mathrm{~N}$ comparison. All but one of the ${ }^{15} \mathrm{~N}$ RADSAT-NG cross sections were within $5 \%$ of the GROUPR cross sections. The ${ }^{15} \mathrm{~N}(\mathrm{n}, \mathrm{np})$ total RADSAT-NG cross section differs from GROUPR by $28.82 \%$. Although the ${ }^{15} \mathrm{~N}$ (n,np) cross section spans four energy groups, the highest energy group composes $99.17 \%$ of the total cross section for the interaction. The RADSAT-NG cross section in the highest energy neutron group was larger than the GROUPR collapsed cross section by $28.82 \%$. As previously noted, there is a known difference in the handling of RADSAT-NG and GROUPR cross sections in the highest and lowest energy groups which has been documented but is still under investigation.

Table 11. Comparison of Total Cross Section for RADSAT-NG and Cross Sections Produced by GROUPR for ${ }^{14} \mathrm{~N}$

\begin{tabular}{|l|l|l|l|}
\hline Cross Section & $\begin{array}{l}\text { RADSAT-NG } \\
\text { Total Cross Section } \\
\text { (Barns) }\end{array}$ & $\begin{array}{l}\text { GROUPR } \\
\text { Total Cross Section } \\
\text { (Barns) }\end{array}$ & $\begin{array}{l}\text { Percent } \\
\text { Difference } \\
\text { (\%) }\end{array}$ \\
\hline${ }^{14} \mathrm{~N}(\mathrm{n}, \mathrm{n})$ & 2.0093 & 2.0270 & 0.87 \\
\hline${ }^{14} \mathrm{~N}(\mathrm{n}, \mathrm{np})$ & 0.1478 & 0.1480 & 0.19 \\
\hline${ }^{14} \mathrm{~N}(\mathrm{n}, \mathrm{nd})$ & 0.0256 & 0.0257 & 0.11 \\
\hline${ }^{14} \mathrm{~N}(\mathrm{n}, \gamma)$ & 5.0332 & 4.9037 & 2.64 \\
\hline${ }^{14} \mathrm{~N}(\mathrm{n}, \mathrm{p})$ & 0.2563 & 0.2579 & 0.62 \\
\hline${ }^{14} \mathrm{~N}(\mathrm{n}, \mathrm{d})$ & 0.0951 & 0.0953 & 0.27 \\
\hline${ }^{14} \mathrm{~N}(\mathrm{n}, \mathrm{t})$ & 0.0668 & 0.0677 & 1.43 \\
\hline${ }^{14} \mathrm{~N}(\mathrm{n}, \alpha)$ & 0.5969 & 0.6004 & 0.57 \\
\hline${ }^{15} \mathrm{~N}(\mathrm{n}, \mathrm{n})$ & 3.9162 & 3.9123 & 0.10 \\
\hline${ }^{15} \mathrm{~N}(\mathrm{n}, 2 \mathrm{n})$ & 0.0295 & 0.0283 & 4.31 \\
\hline${ }^{15} \mathrm{~N}(\mathrm{n}, \mathrm{n} \alpha)$ & 0.0209 & 0.0200 & 4.65 \\
\hline${ }^{15} \mathrm{~N}(\mathrm{n}, \mathrm{np})$ & 0.0005 & 0.0004 & 28.82 \\
\hline${ }^{15} \mathrm{~N}(\mathrm{n}, \mathrm{p})$ & 0.0325 & 0.0326 & 0.37 \\
\hline${ }^{15} \mathrm{~N}(\mathrm{n}, \mathrm{d})$ & 0.0290 & 0.0283 & 0.89 \\
\hline${ }^{15} \mathrm{~N}(\mathrm{n}, \mathrm{t})$ & 0.0315 & 0.0346 & 1.65 \\
\hline${ }^{15} \mathrm{~N}(\mathrm{n}, \alpha)$ & 0.1899 & 0.1914 & 0.80 \\
\hline
\end{tabular}




\subsubsection{Photon Flux within Detector}

Once the induced photon spectrum was calculated for the neutron irradiation of nitrogen using the RADSAT-NG cross sections and the spatially dependent neutron flux obtained from Attila, the RADSAT group generator was used to produced the specialized photon group structure. 166 continuum groups and 133 0.3-keV wide peak groups were assigned using the group generator, one for the 511-keV annihilation peak and the

remaining peak groups 165 most intense gamma rays produced by ${ }^{14} \mathrm{~N}$. A $1 / \sqrt{\boldsymbol{E}}$ energy weighting factor was applied to the continuum groups. This group structure was used to create the photon cross-section library for the photon transport calculation.

A comparison of the volume-averaged gamma flux in the detector for MCNP and RADSAT is shown in Figure 30. A comparison of the 5 highest intensity gamma rays for MCNP and RADSAT show typical agreement to within 3\%, with the statistical uncertainty of the MCNP calculations generally greater than $4 \%$ for those same lines and is shown in Table 12. The nitrogen test case indicates that the RADSAT-NG crosssection creation methods are adequately collapsing and compiling the many crosssections needed for more complex neutron activation isotopes. 


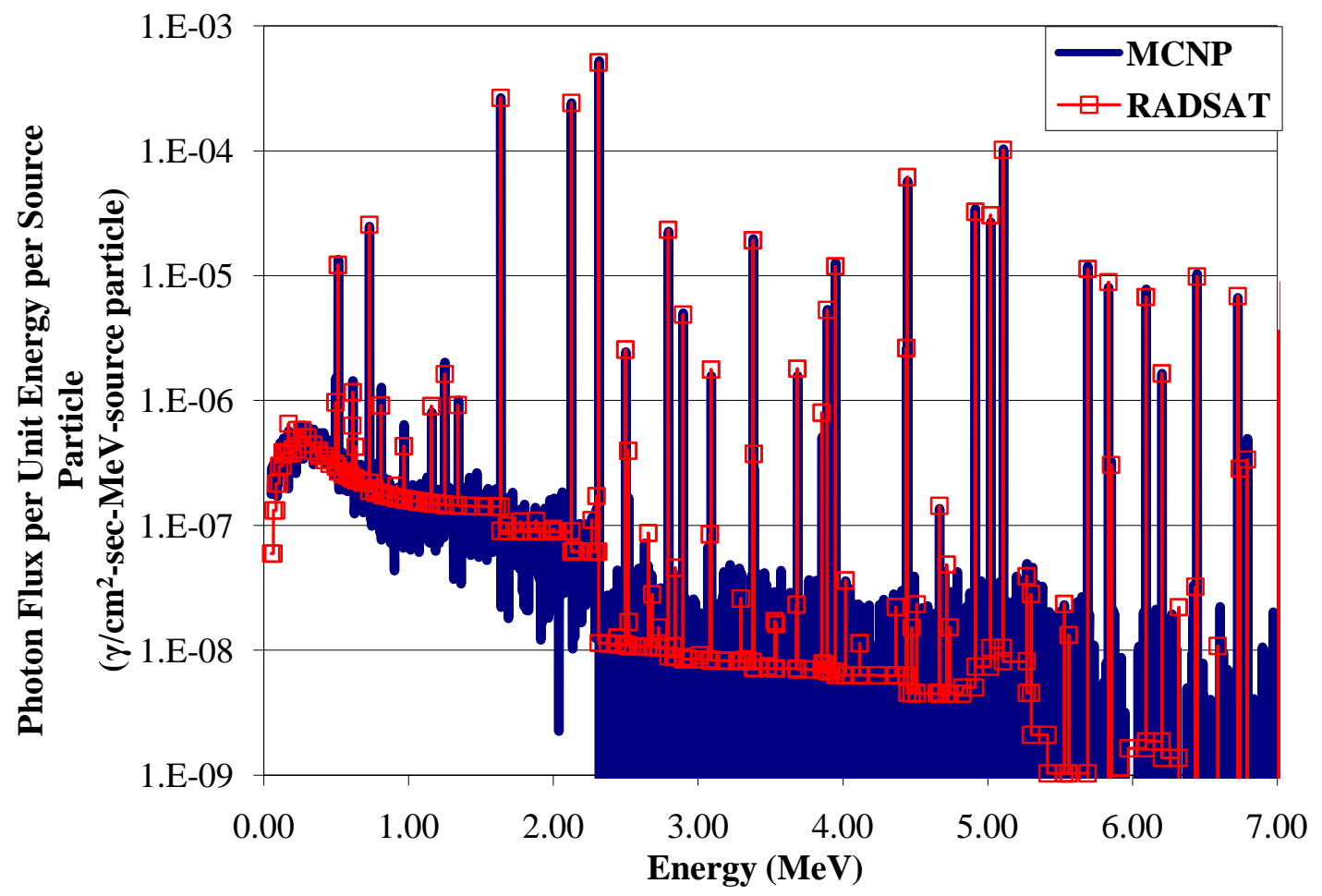

Figure 30. Comparison of Volume-Averaged Photon Flux in Detector using RADSAT and MCNP for Nitrogen

Table 12. Comparison of Photon Flux for Top 5 Most Intense Peaks Produced by the Neutron Irradiation of Nitrogen

\begin{tabular}{|l|l|l|l|l|}
\hline $\begin{array}{l}\text { Gamma-Ray } \\
\text { Energy (MeV) }\end{array}$ & $\begin{array}{l}\text { MCNP } \\
\mathbf{2} \\
\left(\boldsymbol{\gamma} / \mathbf{c m}^{\text {-sec- }}\right. \\
\text { source particle) }\end{array}$ & $\begin{array}{l}\text { MCNP Relative } \\
\text { Uncertainty } \\
\mathbf{( \% )}\end{array}$ & $\begin{array}{l}\text { RADSAT } \\
\mathbf{2} \\
\left(\boldsymbol{\gamma} / \mathbf{c m}^{\text {-sec- }}\right. \\
\text { source particle) }\end{array}$ & $\begin{array}{l}\text { Percent } \\
\text { Difference } \\
\mathbf{( \% )}\end{array}$ \\
\hline 1.635302 & $2.65 \mathrm{E}-07$ & 4.38 & $2.64 \mathrm{E}-07$ & 0.38 \\
\hline 2.12469 & $5.23 \mathrm{E}-07$ & 4.59 & $5.06 \mathrm{E}-07$ & 3.23 \\
\hline 2.312799 & $5.23 \mathrm{E}-07$ & 3.15 & $5.08 \mathrm{E}-07$ & 2.87 \\
\hline 4.444891 & $5.73 \mathrm{E}-08$ & 9.65 & $5.56 \mathrm{E}-08$ & 2.90 \\
\hline 5.105891 & $1.03 \mathrm{E}-07$ & 6.62 & $1.01 \mathrm{E}-07$ & 1.94 \\
\hline Total & $1.86 \mathrm{E}-06$ & 1.65 & $1.82 \mathrm{E}-06$ & 2.17 \\
\hline
\end{tabular}

\subsection{Oxygen}

Oxygen, like nitrogen, is very common in nature, and the induced photon spectrum for the neutron irradiation of oxygen is approximately the same level of complexity as nitrogen. Oxygen, also like nitrogen, is one of the most abundant elements 
on Earth. It composes a larger fraction of the Earth's crust and oceans than nitrogen but a smaller percentage of the air. Oxygen is used for many applications including torches, rocket propellants, chemical weapons, medicine, antifreeze, plastics and fabrics. Due to oxygen's wide range of applications and abundance on Earth, many applications may benefit from the addition of the inclusion of oxygen in the RADSAT-NG photon production cross sections in the library.

\subsubsection{Neutron Flux Comparison}

The first step in completing the coupled neutron-photon calculation was to complete the neutron transport calculation for oxygen. An arbitrary density 0.5 grams per cubic centimeter was modeled to increase the probability of the neutron interaction in the oxygen since pure oxygen is typically only present in low densities (natural density of 0.001429 grams $/ \mathrm{cm}^{3}$ at $\left.0^{\circ} \mathrm{C}[31]\right)$. The total neutron flux within the oxygen cube for Attila is within $1.69 \%$ of the MCNP simulation result and is shown in Table 13. Unlike the Attila simulation which provides an infinite time solution, the MCNP result does not obtain counts in many of the lower energy bins even though the statistical uncertainty for the overall tally is less than $1 \times 10^{-4} \%$. The statistical uncertainty for the $10-\mathrm{eV}$ energy bin is $17.83 \%$, and the statistical uncertainty for the 5-eV energy bin and below is greater than or equal to $29.65 \%$. Only two of the energy bins below $0.145-\mathrm{eV}$ registered any counts at all. A comparison of the volume-averaged neutron flux within the entire oxygen cube obtained from MCNP and Attila using the Kynea3 cross section library is shown in Figure 31. Although the statistical uncertainly below 50-kev is relatively poor on the MCNP calculation, the Attila calculation exhibits a substantial bias. Determining the origin of this bias remains outside of the scope of this body of work, but it is believed 
that the bias is caused by a lack of thermalization of neutrons to this region. Adjusting the density of the problem or the target material to a mixture of poly and oxygen may remedy this bias.

Table 13. Comparison of Attila and MCNP Total Neutron Flux for the Irradiation of Oxygen

\begin{tabular}{|l|l|l|l|}
\hline $\begin{array}{l}\text { MCNP Flux } \\
\text { (Neutron } / \mathbf{c m}^{2} \text {-sec- } \\
\text { source particle) }\end{array}$ & $\begin{array}{l}\text { MCNP Statistical } \\
\text { Uncertainty (\%) }\end{array}$ & $\begin{array}{l}\text { Attila Flux } \\
\text { (Neutron } / \mathbf{c m}^{2} \text {-sec- } \\
\text { source particle) }\end{array}$ & $\begin{array}{l}\text { Percent } \\
\text { Difference (\%) }\end{array}$ \\
\hline $8.21 \times 10^{-4}$ & $<1 \times 10^{-4}$ & $8.34 \mathrm{Ex} 10^{-4}$ & 1.69 \\
\hline
\end{tabular}

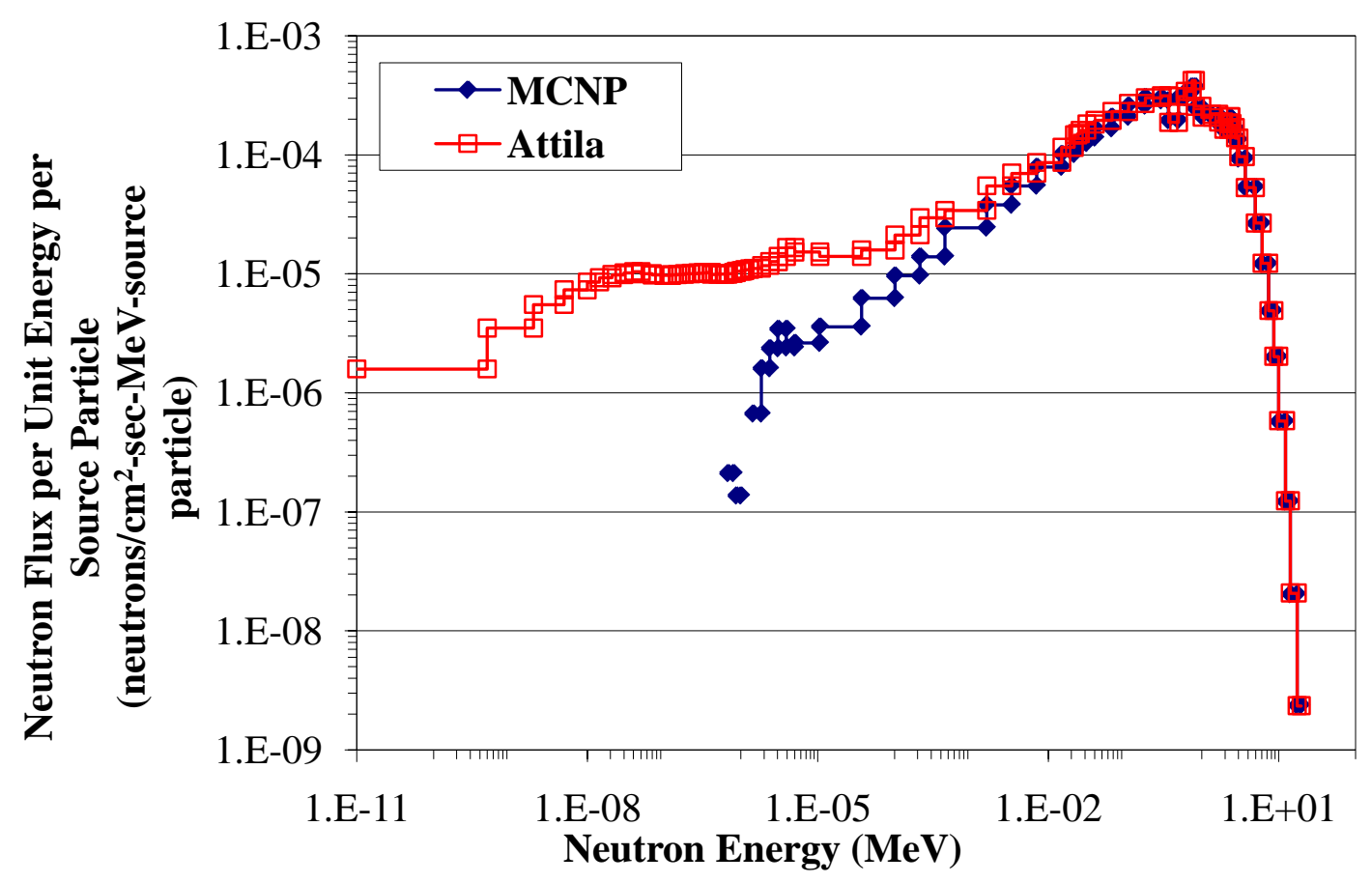

Figure 31. Comparison of Attila and MCNP Neutron Flux for the Irradiation of Oxygen 


\subsubsection{Cross Sections}

The cross section for oxygen is similar to that of nitrogen in that it does not contain any unresolved resonance features. Oxygen is composed of three naturally occurring isotopes ${ }^{16} \mathrm{O},{ }^{17} \mathrm{O}$ and ${ }^{18} \mathrm{O}(99.762 \%, 0.038 \%$ and $0.2 \%$ respectively). There is no neutron cross section for ${ }^{18} \mathrm{O}$, so it will not be included in these calculations. Additionally, the ENDF/B-VI cross section for ${ }^{17} \mathrm{O}$ does not contain any photon production cross section data. Thus, the only isotope of oxygen used for the photon production calculations is ${ }^{16} \mathrm{O} .{ }^{16} \mathrm{O}$ has eight gamma-ray producing reactions in the neutron energy range included in the Kynea3 neutron energy group structure, producing a total of 51 discrete and 4 continua gamma rays. A list of the gamma-ray producing interactions in ${ }^{16} \mathrm{O}$ and the gamma rays produced by each interaction is shown in Table 14. The ${ }^{16} \mathrm{O}$ RADSAT-NG cross sections were collapsed from the ENDF/B-VI cross section data at 300K. A comparison of the RADSAT-NG and GROUPR radiative capture and $(n, \alpha)$ cross sections for ${ }^{16} \mathrm{O}$ are shown in Figure 32 and Figure 33, respectively.

Table 14. Discrete Gamma Rays Produced by ${ }^{16} \mathrm{O}$

\begin{tabular}{|c|c|}
\hline Reaction & Gamma Ray Energy (MeV) \\
\hline$\left(n, n^{\prime}\right)$ & $\begin{array}{l}0.51,0.8677,0.986957,1.75505,1.9548,2.2081,2.742007,2.8225,3.84015, \\
4.1629,4.950107,6.129893,6.9171,7.11685,8.8719\end{array}$ \\
\hline$(\mathrm{n}, \mathrm{n} \alpha)$ & 4.438 \\
\hline$(\mathrm{n}, \gamma)$ & $0.8708,1.088,2.1842,3.2722$ \\
\hline$(n, p)$ & $0.1201,0.2774,0.297,0.3975$ \\
\hline$(\mathrm{n}, \mathrm{d})$ & $\begin{array}{l}1.0117,1.1577,1.4163,1.885,1.9889,2.0023,2.031,2.2475,2.297,3.014, \\
3.3013,5.2701,5.2988,6.3239,7.3011,7.5671,8.3128,8.5714\end{array}$ \\
\hline$(\mathrm{n}, \mathrm{t})$ & $1.635302,2.312798,3.9481$ \\
\hline$(n, \alpha)$ & $0.1693,0.5951,0.7644,3.0894,3.6845,3.8538$ \\
\hline
\end{tabular}




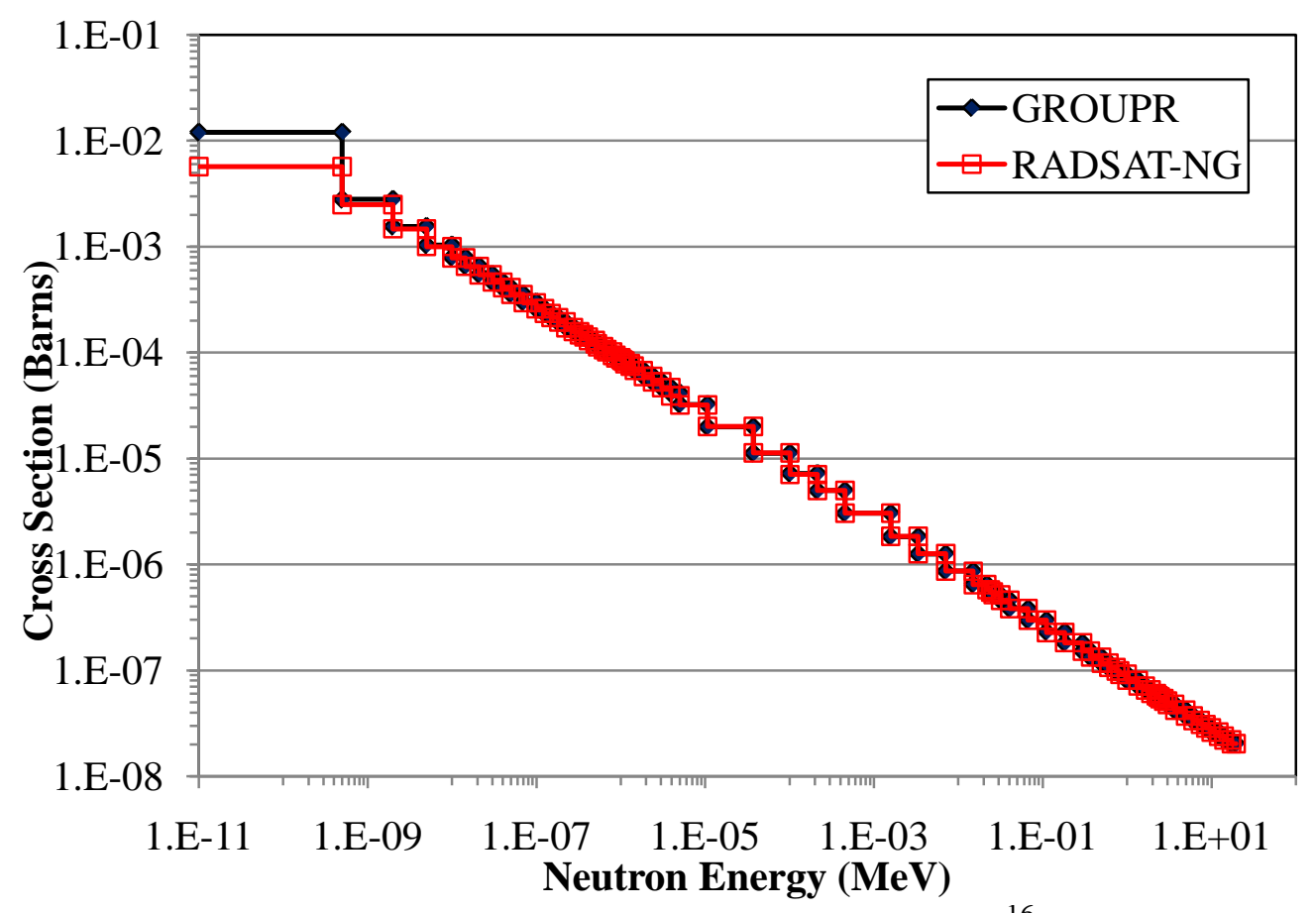

Figure 32. Comparison of the GROUPR and RADSAT-NG ${ }^{16} \mathrm{O}$ Radiative Capture Photon Production Cross Section

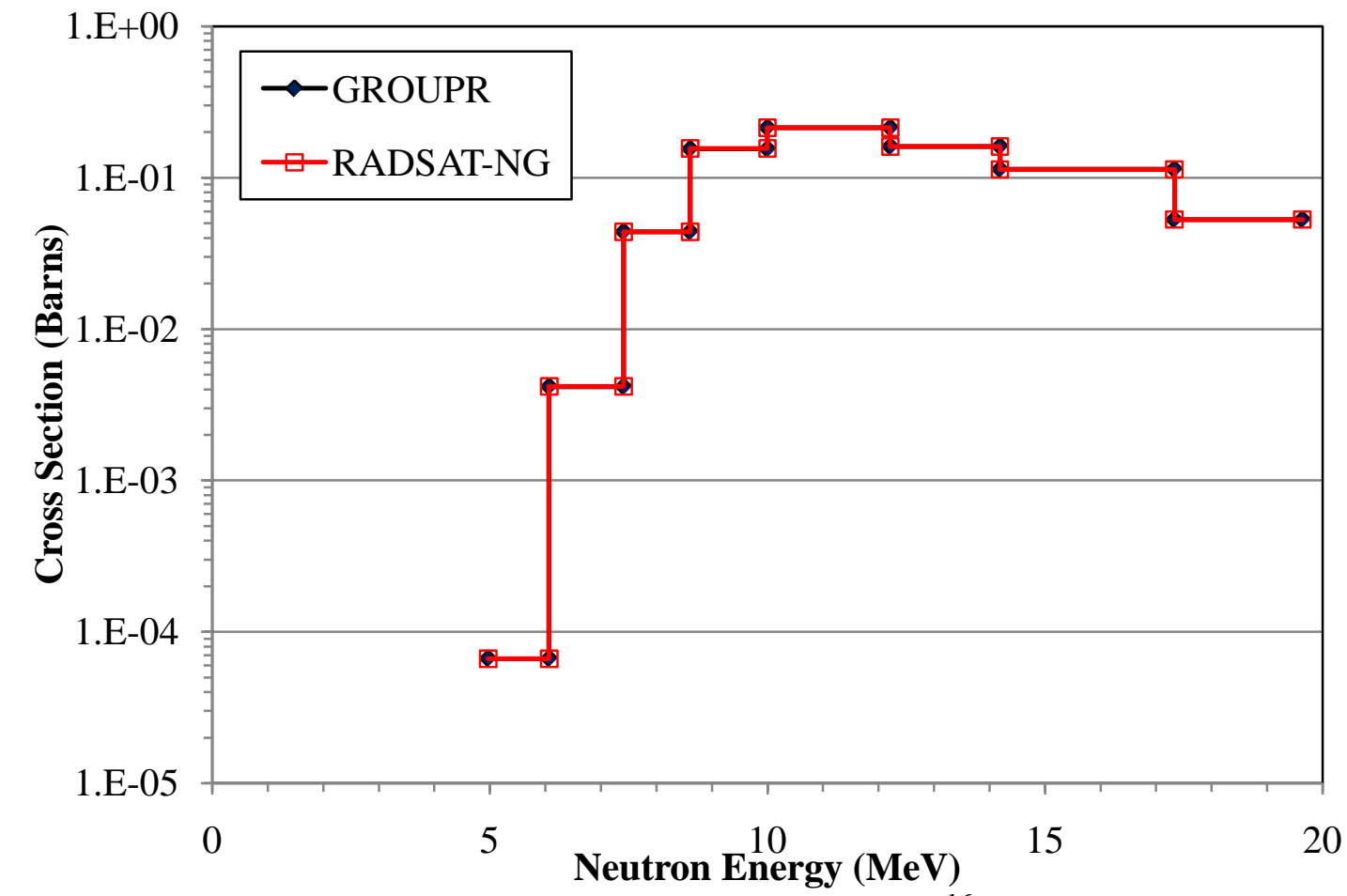

Figure 33. Comparison of the GROUPR and RADSAT-NG ${ }^{16} \mathrm{O}(\mathrm{n}, \alpha)$ Photon Production Cross Section 
The comparison of the RADSAT-NG cross sections and the cross sections collapsed using GROUPR shows reasonably good agreement for ${ }^{16} \mathrm{O}$ as shown in Table 17. The difference in ${ }^{16} \mathrm{O}$ radiative capture cross section is due to a difference in the lowest energy group. The RADSAT-NG radiative capture cross section for the lowest energy group is a factor of 2 lower than the GROUPR collapsed cross section. The remainder of the cross section agreed with the GROUPR collapsed cross section.

The large percent difference of $19.96 \%$ for the $(n, n 3 \alpha)$ cross section is due to the fact that the cross section is present in only one energy bin. The RADSAT-NG and GROUPR method rarely calculate the same value for the lowest and highest cross section bins, so when a cross section relies on the calculation of only one bin the codes do not agree. The reason for this discrepancy is still under investigation. The RADSAT-NG cross section library calculates a cross section in a second energy bin, but its value is 10 orders of magnitude smaller than the cross section in highest neutron energy group which is believed to be below the minimum cross section value calculated by GROUPR. A similar scenario is true for the (n,np) cross section which would account for the $6.93 \%$ difference in the RADSAT-NG and GROUPR cross sections.

The $(n, 2 \alpha)$ cross section was not collapsed by GROUPR at all even though the cross section is present in the Kynea3 energy range. A comparison of the continuous energy ACER photon production cross section to the RADSAT-NG photon production cross section for the $(n, 2 \alpha)$ reaction is shown in Figure 34. It is hypothesized that GROUPR did not collapse this particular reaction due to the small magnitude of the cross section. 
Table 15. Comparison of Total Cross Section for RADSAT-NG and Cross Sections Produced by GROUPR for ${ }^{16} \mathrm{O}$

\begin{tabular}{|l|l|l|l|}
\hline Cross Section & $\begin{array}{l}\text { RADSAT-NG } \\
\text { Total Cross Section } \\
\text { (Barns) }\end{array}$ & $\begin{array}{l}\text { GROUPR } \\
\text { Total Cross Section } \\
\text { (Barns) }\end{array}$ & $\begin{array}{l}\text { Percent } \\
\text { Difference } \\
\text { (\%) }\end{array}$ \\
\hline$\left.{ }^{16} \mathrm{O}(\mathrm{n}, \mathrm{n})\right)$ & $2.39 \mathrm{E}+00$ & $2.39 \mathrm{E}+00$ & 0.04 \\
\hline${ }^{16} \mathrm{O}(\mathrm{n}, \mathrm{n} \alpha)$ & $1.96 \mathrm{E}-01$ & $1.95 \mathrm{E}-01$ & 0.16 \\
\hline${ }^{16} \mathrm{O}(\mathrm{n}, \mathrm{n} 3 \alpha)$ & $5.70 \mathrm{E}-08$ & $4.75 \mathrm{E}-08$ & 19.96 \\
\hline${ }^{16} \mathrm{O}(\mathrm{n}, \mathrm{np})$ & $4.13 \mathrm{E}-05$ & $3.86 \mathrm{E}-05$ & 6.93 \\
\hline${ }^{16} \mathrm{O}(\mathrm{n}, \gamma)$ & $1.71 \mathrm{E}-02$ & $2.39 \mathrm{E}-02$ & 28.35 \\
\hline${ }^{16} \mathrm{O}(\mathrm{n}, \mathrm{p})$ & $1.29 \mathrm{E}-01$ & $1.29 \mathrm{E}-01$ & 0.07 \\
\hline${ }^{16} \mathrm{O}(\mathrm{n}, \mathrm{d})$ & $1.85 \mathrm{E}-02$ & $1.85 \mathrm{E}-02$ & 0.50 \\
\hline${ }^{16} \mathrm{O}(\mathrm{n}, \mathrm{t})$ & $6.65 \mathrm{E}-04$ & $6.36 \mathrm{E}-04$ & 4.47 \\
\hline${ }^{16} \mathrm{O}(\mathrm{n}, \alpha)$ & $7.44 \mathrm{E}-01$ & $7.44 \mathrm{E}-01$ & 0.02 \\
\hline${ }^{16} \mathrm{O}(\mathrm{n}, 2 \alpha)$ & $1.37 \mathrm{E}-13$ & $0.00 \mathrm{E}+00$ & N/A \\
\hline
\end{tabular}

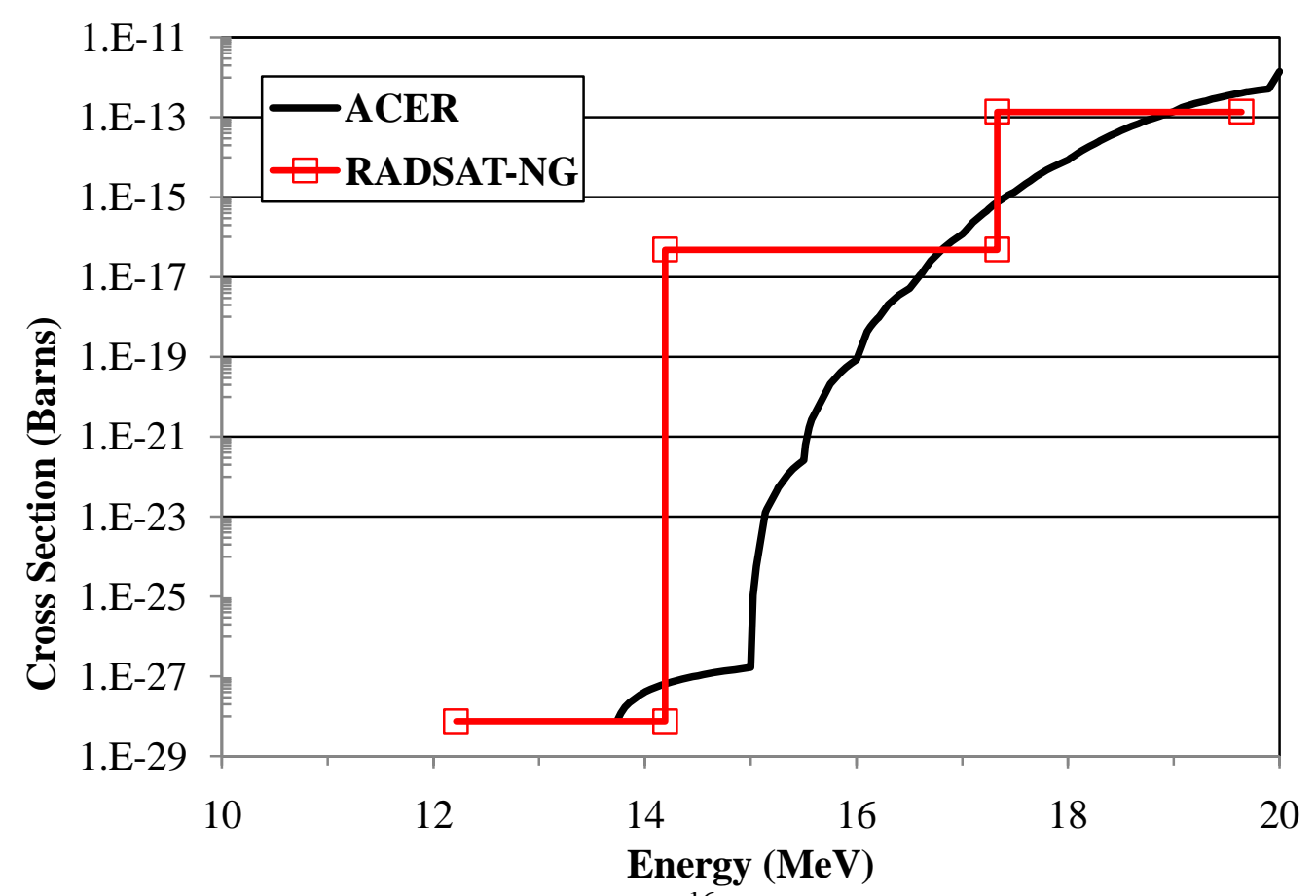

Figure 34. Comparison of Energy-dependent ${ }^{16} \mathrm{O}(\mathrm{n}, 2 \alpha)$ Photon Production Cross Section for RADSAT-NG, GROUPR and ACER 


\subsubsection{Photon Flux within Detector}

Using the RADSAT-NG cross sections and the spatially dependent neutron flux obtained from Attila, the induced photon spectrum was calculated for the neutron irradiation of oxygen. The RADSAT group generator then was used to produce the specialized photon group structure containing 248 continuum groups and $520.3-\mathrm{keV}$ wide groups, one for the 511-keV annihilation peak and for the remaining groups 51 most intense gamma rays produced by ${ }^{16} \mathrm{O}$ using a $1 / \sqrt{E}$ energy weighting factor for the continuum energy groups.

The volume-averaged gamma flux in the detector from the neutron irradiation of oxygen calculated using the RADSAT method agrees well with the results obtained from the MCNP simulation as shown in Figure 35. The total photon flux within the detector is within $6.4 \%$ of the MCNP result. The $0.511-\mathrm{MeV}$ annihilation line is underestimated using the RADSAT method, and the continuum region below the $0.511-\mathrm{MeV}$ line does not quite obtain the same shape as the MCNP simulation. The 5 most intense gamma rays for MCNP and RADSAT show typical agreement to within 3\%. The statistical uncertainty of the MCNP simulation for the same gamma ray emissions is equal to or greater than the percent difference between the MCNP and RADSAT calculations as shown in Table 16. 


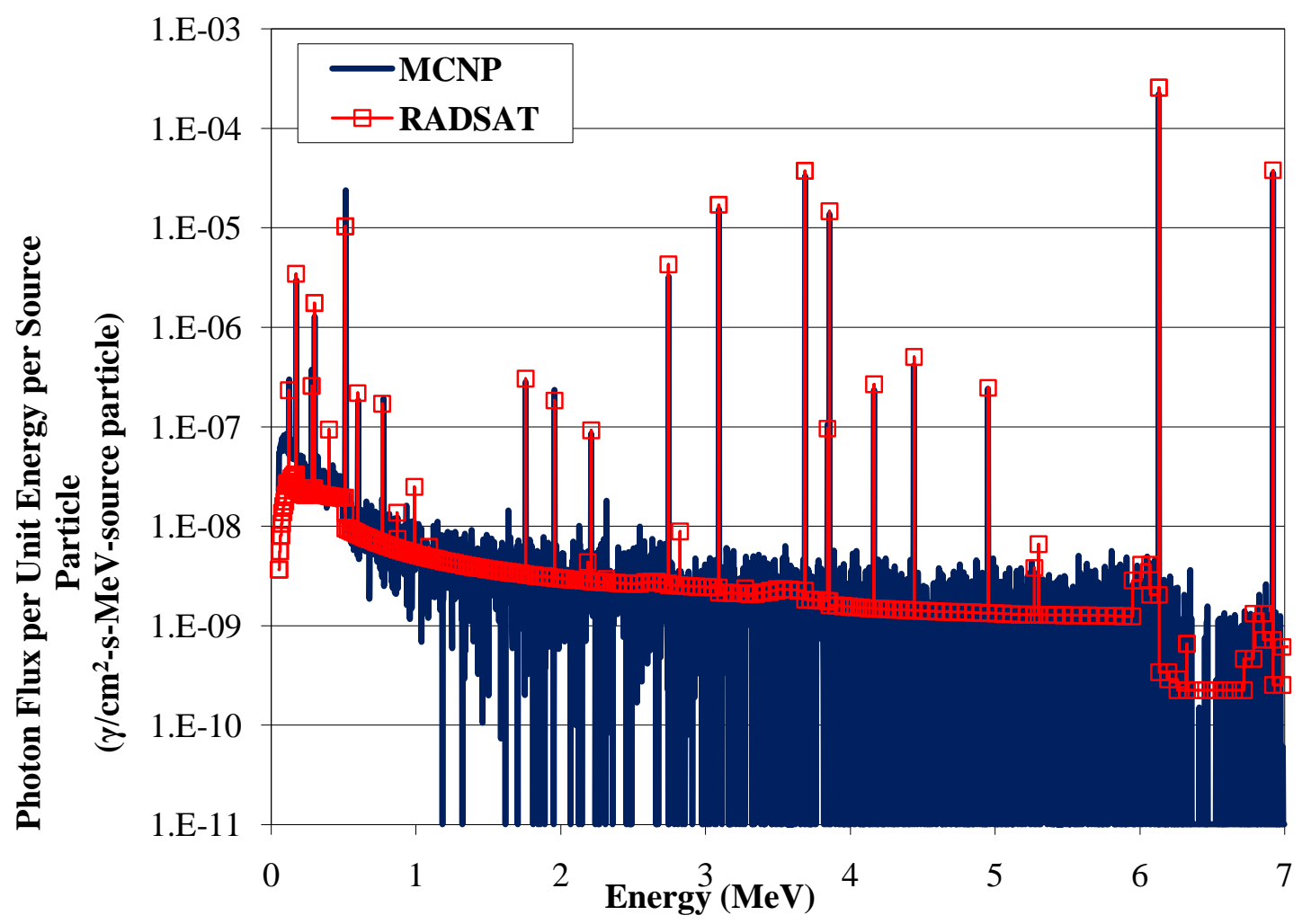

Figure 35. Comparison of Volume-Averaged Photon Flux in Detector using RADSAT and MCNP for Oxygen

Table 16. Comparison of Photon Flux for Top 6 Most Intense Peaks Produced by the Neutron Irradiation of Oxygen

\begin{tabular}{|c|c|c|c|c|}
\hline $\begin{array}{l}\text { Gamma-Ray } \\
\text { Energy (MeV) }\end{array}$ & $\begin{array}{l}\text { MCNP } \\
\left(\gamma / \mathrm{cm}^{2} \text {-sec- }\right. \\
\text { source particle) }\end{array}$ & $\begin{array}{l}\text { MCNP Relative } \\
\text { Uncertainty (\%) }\end{array}$ & $\begin{array}{l}\text { RADSAT } \\
\left(\gamma / \mathrm{cm}^{2}-\mathrm{sec}-\right. \\
\text { source particle) }\end{array}$ & $\begin{array}{l}\text { Percent } \\
\text { Difference } \\
\text { (\%) } \\
\end{array}$ \\
\hline 2.742007 & $1.22 \mathrm{E}-09$ & 9.68 & $1.28 \mathrm{E}-09$ & 5.26 \\
\hline 3.0894 & $3.98 \mathrm{E}-09$ & 5.10 & $3.88 \mathrm{E}-09$ & 2.38 \\
\hline 3.6845 & $\begin{array}{l}8.70 \mathrm{E}-09 \\
\end{array}$ & 3.24 & $8.53 \mathrm{E}-09$ & 2.03 \\
\hline 3.8538 & $3.60 \mathrm{E}-09$ & 5.35 & $3.35 \mathrm{E}-09$ & 0.41 \\
\hline 6.129893 & \begin{tabular}{|c|}
$5.84 \mathrm{E}-08$ \\
\end{tabular} & 1.25 & $5.85 \mathrm{E}-08$ & 0.15 \\
\hline 6.9171 & 9.34E-09 & 3.31 & $8.62 \mathrm{E}-09$ & 3.37 \\
\hline Total & $\begin{array}{l}1.46 \mathrm{E}-07 \\
\end{array}$ & 0.90 & $1.55 \mathrm{E}-07$ & 6.40 \\
\hline
\end{tabular}




\subsection{Iron}

Iron was selected as a benchmarking material due to its common presence in many common applications. Iron is the main material component of steel. Also, iron is commonly used as a structural component for buildings, cars, planes, ships, office supplies, and computers. Many of these items could be exposed to neutron fields, so understand the neutron-induced photon spectrum is important to many detector scenarios.

\subsubsection{Neutron Flux Comparison}

The neutron flux comparison was completed to test the accuracy of the Kynea3 cross section for iron. The iron was modeled at $7.874 \mathrm{grams} / \mathrm{cm}^{3}$ which is the natural density of iron at $300 \mathrm{~K}$ [31]. The total neutron flux within the iron cube for Attila is within $1.25 \%$ of the MCNP simulation result and is shown in Table 17. The Attila was able to resolve the flux at the lower energy ranges causing the total flux to be higher than the flux obtained by MCNP since it did not obtain any counts in the lower energy bins at all. Although the MCNP statistical uncertainty for the tally is less than $1 \times 10^{-4} \%$, the statistical uncertainty for the $37.3-\mathrm{eV}$ energy bin is $8.49 \%$, and the statistical uncertainty for the 5-eV energy bin and below is greater than or equal to $38.52 \%$. A graphical comparison of the volume-averaged neutron flux within the nitrogen cube for MCNP and Attila is shown in Figure 36. As with the other neutron transport calculations that do not contain many thermal neutrons, the Attila calculation exhibits a systematic bias below 50-keV when compared to MCNP for the neutron flux. 
Table 17. Comparison of Attila and MCNP Total Neutron Flux for the Irradiation of Iron

\begin{tabular}{|l|l|l|l|}
\hline $\begin{array}{l}\text { MCNP Flux } \\
\text { (Neutron /cm } \\
\text { source particle) }\end{array}$ & $\begin{array}{l}\text { MCNP Statistical } \\
\text { Error (\%) }\end{array}$ & $\begin{array}{l}\text { Attila Flux } \\
\text { (Neutron } / \mathbf{c m}^{2} \text {-sec- } \\
\text { source particle) }\end{array}$ & $\begin{array}{l}\text { Percent } \\
\text { Difference } \\
\text { (\%) }\end{array}$ \\
\hline $7.60 \times 10^{-4}$ & $>1 \times 10^{-4} \%$ & $7.77 \times 10^{-4}$ & $1.25 \%$ \\
\hline
\end{tabular}

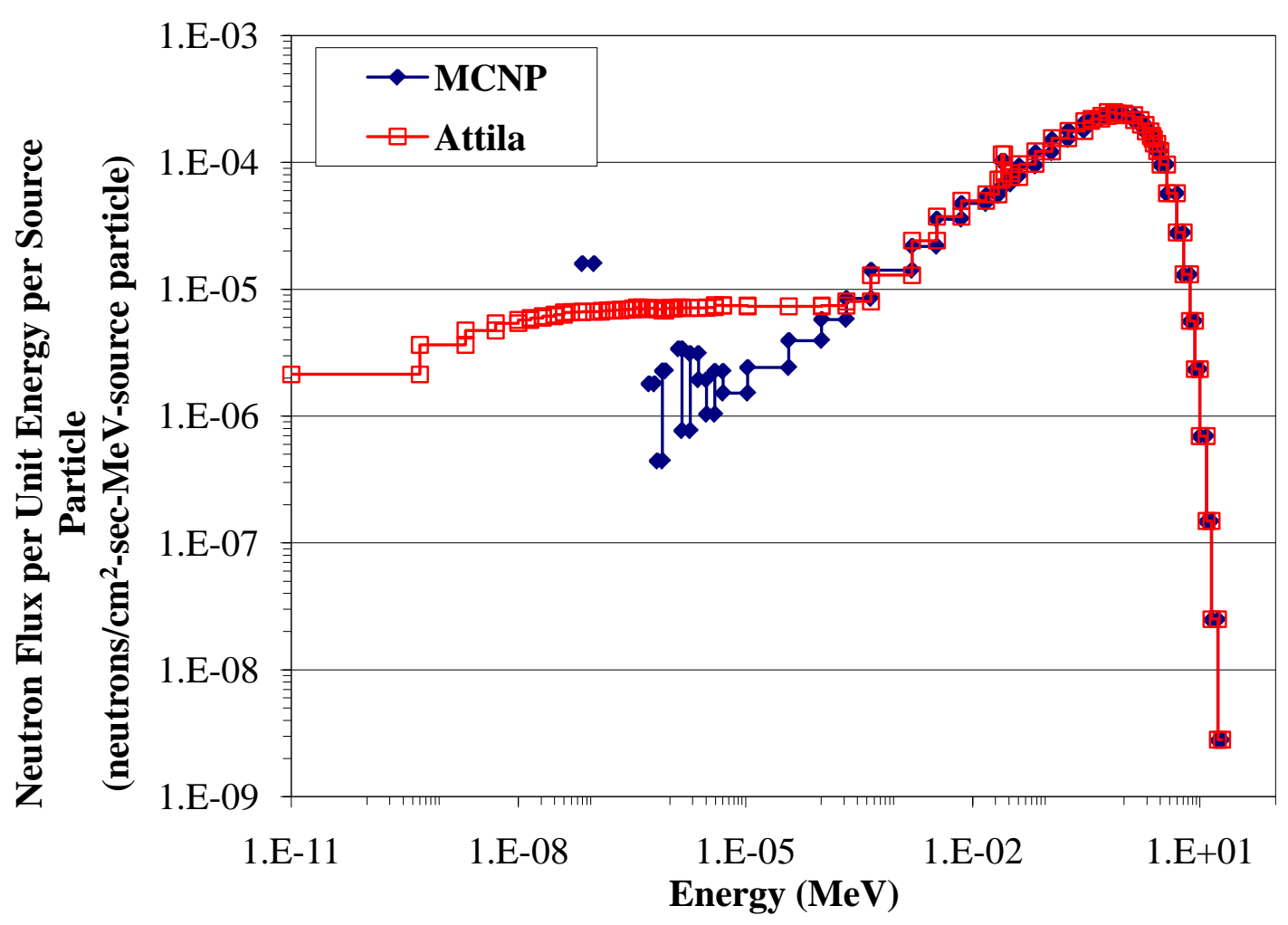

Figure 36. Comparison of Attila and MCNP Neutron Flux for the Irradiation of Iron

\subsubsection{Cross Sections}

Although the ENDF/B-VI cross sections for iron do not contain any unresolved resonances, they do contain resolved resonances making them the most difficult cross sections in this document to collapse. Iron has four naturally occurring isotopes ${ }^{54} \mathrm{Fe}$, ${ }^{56} \mathrm{Fe},{ }^{57} \mathrm{Fe}$ and ${ }^{58} \mathrm{Fe}$, with corresponding natural abundances of $5.8 \%, 91.72 \%, 2.2 \%$ and $0.28 \%$. Unfortunately the GROUPR subroutine produces an energy grid error when 
collapsing ${ }^{57} \mathrm{Fe}$, so the comparison of the RADSAT-NG and GROUPR cross sections for ${ }^{57} \mathrm{Fe}$ has been omitted. ${ }^{54} \mathrm{Fe}$ has 7 gamma producing reactions which result in 52 discrete gamma rays and 7 continua; ${ }^{56} \mathrm{Fe}$ has 7 gamma producing reactions which create in 308 discrete gamma rays and 7 continua; ${ }^{57} \mathrm{Fe}$ has 7 gamma producing reactions which create in 112 discrete gamma rays and 7 continua; and ${ }^{58} \mathrm{Fe}$ has 14 gamma producing reactions resulting in 143 discrete gamma rays and 7 continua. Table 18 contains a list of the gamma producing interactions in iron and the gamma rays produced by each interaction. The ${ }^{54} \mathrm{Fe},{ }^{56} \mathrm{Fe}$ and ${ }^{58} \mathrm{Fe}$ RADSAT-NG cross sections were collapsed from the ENDF/BVI cross section data at 300K. A comparison of the RADSAT-NG and GROUPR radiative capture cross sections for ${ }^{54} \mathrm{Fe},{ }^{56} \mathrm{Fe}$ and ${ }^{58} \mathrm{Fe}$ are shown in Figure 37, Figure 38, and Figure 39, respectively.

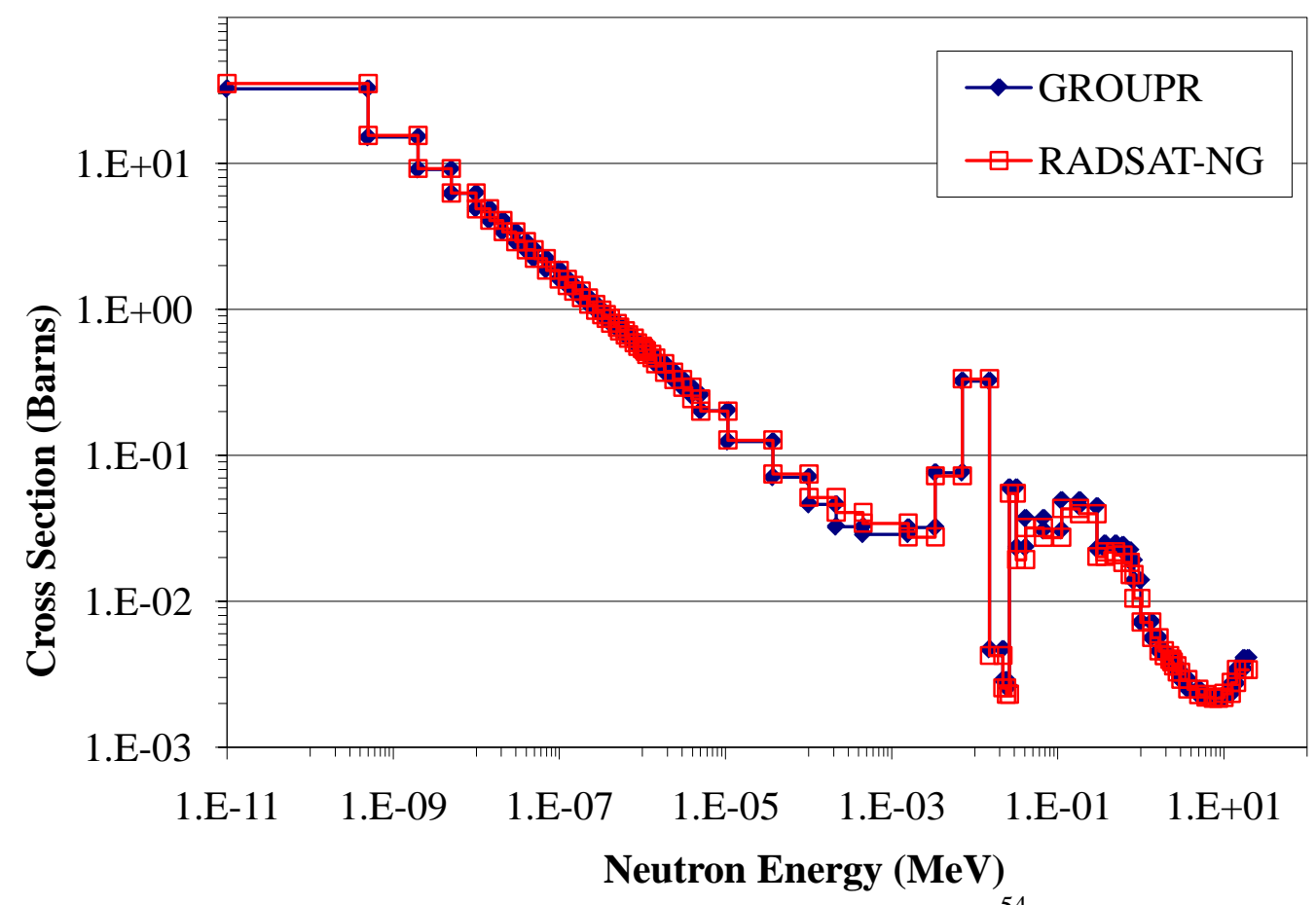

Figure 37. Comparison of the GROUPR and RADSAT-NG ${ }^{54} \mathrm{Fe}$ Radiative Capture Photon Production Cross Section 
Table 18. Discrete Gamma Rays Produced by Iron

\begin{tabular}{|c|c|c|}
\hline Isotope & Reaction & Gamma Rays (MeV) \\
\hline${ }^{54} \mathrm{Fe}$ & $\left(\mathrm{n}, \mathrm{n}^{\prime}\right)$ & $0.412,0.757,1.13,1.153,1.408,1.551,1.758,1.887,2.959,3.166$ \\
\hline${ }^{54} \mathrm{Fe}$ & $(\mathrm{n}, \gamma)$ & $\begin{array}{l}\text { 9.2978, 8.8864, 7.385, 7.363, 7.2463, 6.8268, 6.617, 6.2689, } \\
5.7454,5.5075,5.3911,4.8028,4.707,4.5896,4.535,4.495 \\
4.455,4.18,4.012,3.96,3.906,3.792,3.552,3.508,3.38,3.04 \\
3.028,3.005,2.873,2.791,2.67,2.618,2.47,2.052,1.918,1.872 \\
1.638,1.506,1.315,1.24,0.931,0.412\end{array}$ \\
\hline 56 $\mathrm{Fe}$ & $\left(\mathrm{n}, \mathrm{n}^{\prime}\right)$ & $\begin{array}{l}0.368,0.734,0.787,0.847,0.952,0.977,1.038,1.061,1.089,1.14, \\
1.16,1.174,1.175,1.238,1.272,1.303,1.335,1.36,1.442,1.459, \\
1.462,1.64,1.671,1.772,1.811,1.852,1.964,2.015,2.035,2.095, \\
2.113,2.273,2.373,2.425,2.523,2.598,2.602,2.658,2.755,2.76, \\
2.96,2.985,3.01,3.202,3.253,3.273,3.37,3.449,3.451,3.455, \\
3.548,3.554,3.602,3.611,3.663,3.832\end{array}$ \\
\hline${ }^{56} \mathrm{Fe}$ & $(\mathrm{n}, \gamma)$ & $\begin{array}{l}7.6456,7.6312,7.2788,7.199,6.742,6.7174,6.5485,6.3805, \\
6.2764,6.2194,6.1293,6.1021,6.0184,5.992,5.9204,5.9014, \\
5.7849,5.7306,5.3258,5.318,5.1797,5.0474,5.0421,4.9483, \\
4.914,4.8566,4.8455,4.84,4.8256,4.8098,4.724,4.687,4.6751, \\
4.6593,4.5974,4.5555,4.4625,4.4182,4.4057,4.3783,4.3238, \\
4.2745,4.218,4.2102,4.1948,4.0733,3.991,3.9817,3.9553, \\
3.9215,3.854,3.8424,3.7766,3.7236,3.7109,3.6894,3.663, \\
3.649,3.6413,3.6102,3.5086,3.5045,3.4364,3.4129,3.3563, \\
3.2911,3.267,3.2393,3.2253,3.186,3.1669,3.1031,3.0751, \\
3.0609,3.0479,3.0276,3.0147,2.9703,2.954,2.9502,2.9434, \\
2.9358,2.8737,2.8354,2.8325,2.8215,2.815,2.7559,2.753, \\
2.7342,2.7212,2.7046,2.6966,2.6916,2.6825,2.6543,2.6189, \\
2.6031,2.5981,2.582,2.5743,2.5624,2.5371,2.534,2.5262, \\
2.517,2.5072,2.4908,2.486,2.4802,2.466,2.4621,2.4243, \\
2.4151,2.4074,2.3918,2.3853,2.3517,2.3489,2.246,2.2162, \\
2.2068,2.2027,2.1982,2.1928,2.1866,2.1647,2.1515,2.1386, \\
2.1295,2.1134,2.1045,2.1013,2.097,2.0919,2.0812,2.0689, \\
2.0662,2.0457,2.0397,2.0332,1.991,1.987,1.9821,1.9764, \\
1.9734,1.9653,1.9431,1.9318,1.9276,1.8995,1.8559,1.8513, \\
1.8419,1.8364,1.8289,1.8259,1.8129,1.8105,1.8023,1.7601, \\
1.7253,1.7224,1.7172,1.7102,1.705,1.7008,1.6973,1.691, \\
1.6746,1.6721,1.6555,1.646,1.627,1.6128,1.5846,1.506, \\
1.4924,1.4872,1.4609,1.4574,1.447,1.4356,1.4302,1.412, \\
1.3817,1.3716,1.3691,1.3587,1.3556,1.3518,1.3452,1.3055, \\
1.3009,1.284,1.2823,1.2633,1.2606,1.2555,1.251,1.2186, \\
1.2154,1.1973,1.186,1.1595,1.1198,1.1156,1.1109,1.0773, \\
1.0439,1.0411,1.0264,1.022,1.019,1.0069,0.9918,0.9882, \\
0.9771,0.942,0.92085,0.89828,0.88478,0.87075,0.8495, \\
0.8379,0.83491,0.8186,0.80309,0.7494,0.74731,0.7351,0.723, \\
0.7064,0.7034,0.69203,0.65756,0.60354,0.6013,0.59863, \\
0.57509,0.56992,0.56419,0.4601,0.36675,0.35236,0.33954, \\
0.3359,0.2511,0.23029,0.21187,0.13652,0.12208\end{array}$ \\
\hline
\end{tabular}


Table 18 (continued).

\begin{tabular}{|c|c|c|}
\hline${ }^{57} \mathrm{Fe}$ & $\left(\mathrm{n}, \mathrm{n}^{\prime}\right)$ & $\begin{array}{l}0.993,0.87,0.706,0.692,0.64,0.569,0.367,0.353,0.339,0.23, \\
0.137,0.123,0.014\end{array}$ \\
\hline${ }^{57} \mathrm{Fe}$ & $(\mathrm{n}, \gamma)$ & $\begin{array}{l}10.0441,9.2337,8.3697,7.2622,7.163,6.9607,6.84,6.5064, \\
\text { 6.4143, } 6.1631,6.0287,5.9056,5.89,5.747,5.7218,5.6916, \\
5.6002,5.4939,5.241,5.223,5.212,5.092,5.044,5.001,4.889, \\
4.8239,4.789,4.7498,4.712,4.6265,4.592,4.521,4.506,4.483, \\
4.443,4.411,4.38,4.342,4.3221,4.2981,4.189,4.185,4.1399, \\
4.08,4.062,4.006,3.987,3.952,3.8818,3.74,3.54,3.486,3.326, \\
3.28,3.205,3.183,3.162,3.071,2.876,2.82,2.781,2.726,2.5135, \\
2.4906,2.4669,2.4335,2.2733,2.1376,2.0655,1.971,1.8625, \\
1.6742,1.6625,1.6572,1.4677,1.4463,1.3225,1.306,1.2927, \\
1.269,1.2668,1.2602,1.2507,1.2387,1.1646,1.1073,1.0974, \\
0.8982,0.8636,0.8544,0.8105,0.5247,0.4593,0.4109,0.2781, \\
0.2526,0.2431,0.2387,0.2336\end{array}$ \\
\hline${ }^{58} \mathrm{Fe}$ & $\left(\mathrm{n}, \mathrm{n}^{\prime}\right)$ & $0.811,0.864,1.675$ \\
\hline${ }^{58} \mathrm{Fe}$ & $(\mathrm{n}, \gamma)$ & $\begin{array}{l}\text { 6.5809, 6.2936, 6.2284, 6.1044, 6.097, 6.0127, 5.8542, 5.6722, } \\
5.6117,5.5739,5.5653,5.4195,5.3833,5.3754,5.3691,5.2043, \\
5.136,5.0092,4.9232,4.7638,4.7578,4.7295,4.6618,4.6287, \\
4.6189,4.508,4.4187,4.26,4.1643,4.1334,4.126,4.1142, \\
4.0725,4.0357,4.0115,3.862,3.824,3.7705,3.7573,3.6644, \\
3.59,3.5321,3.5234,3.513,3.5022,3.4778,3.4226 .3 .3408, \\
3.3371,3.2395,3.2003,3.1964,3.1292,3.114,3.1084,3.0979, \\
3.0814,3.0704,3.0571,2.9666,2.9482,2.9162,2.9086,2.8964, \\
\text { 2.8726, 2.7516, 2.6354, 2.5784, 2.5332, } 2.5051,2.4943,2.4478, \\
2.4286,2.3616,2.3397,2.3224,2.2793,2.2409,2.1602,2.1382, \\
2.103,2.091,2.084,1.9619,1.9568,1.9187,1.9043,1.7495, \\
1.7303,1.7221,1.7199,1.6476,1.5988,1.5699,1.5517,1.5488, \\
1.5448,1.4773,1.4683,1.3762,1.3484,1.3233,1.2736,1.2355, \\
1.2112,1.1925,1.1622,1.1369,1.0621,1.0591,1.0488,0.9689, \\
0.87512,0.84124,0.8269,0.7769,0.767,0.75692,0.7274,0.7109, \\
0.6997,0.69719,0.6886,0.6706,0.6429,0.6273,0.6131,0.6107, \\
0.60538,0.5912,0.57081,0.5525,0.5374,0.465 .0 .43943,0.3791, \\
0.3747,0.28703,0.2804\end{array}$ \\
\hline
\end{tabular}




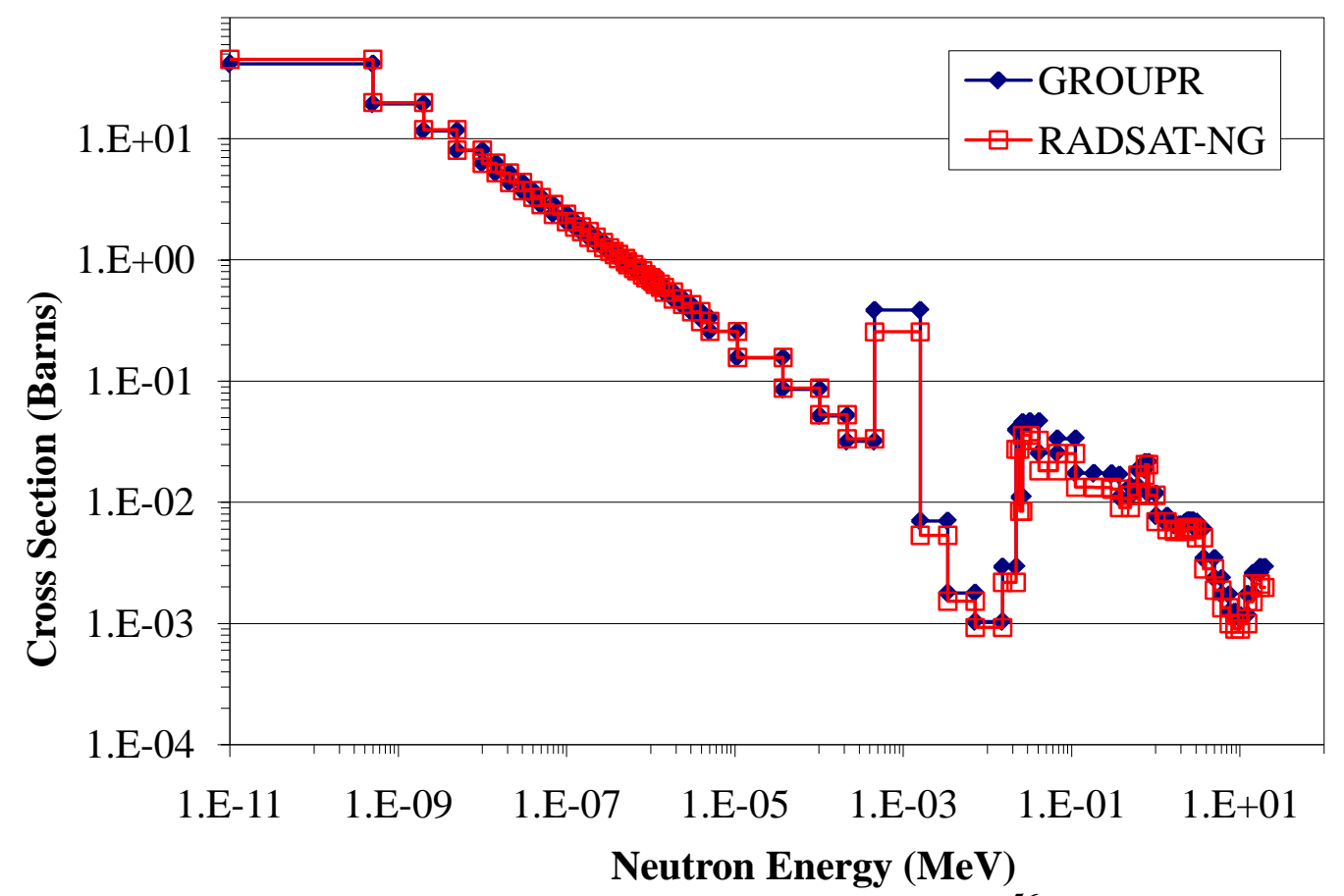

Figure 38. Comparison of the GROUPR and RADSAT-NG ${ }^{56} \mathrm{Fe}$ Radiative Capture Photon Production Cross Section

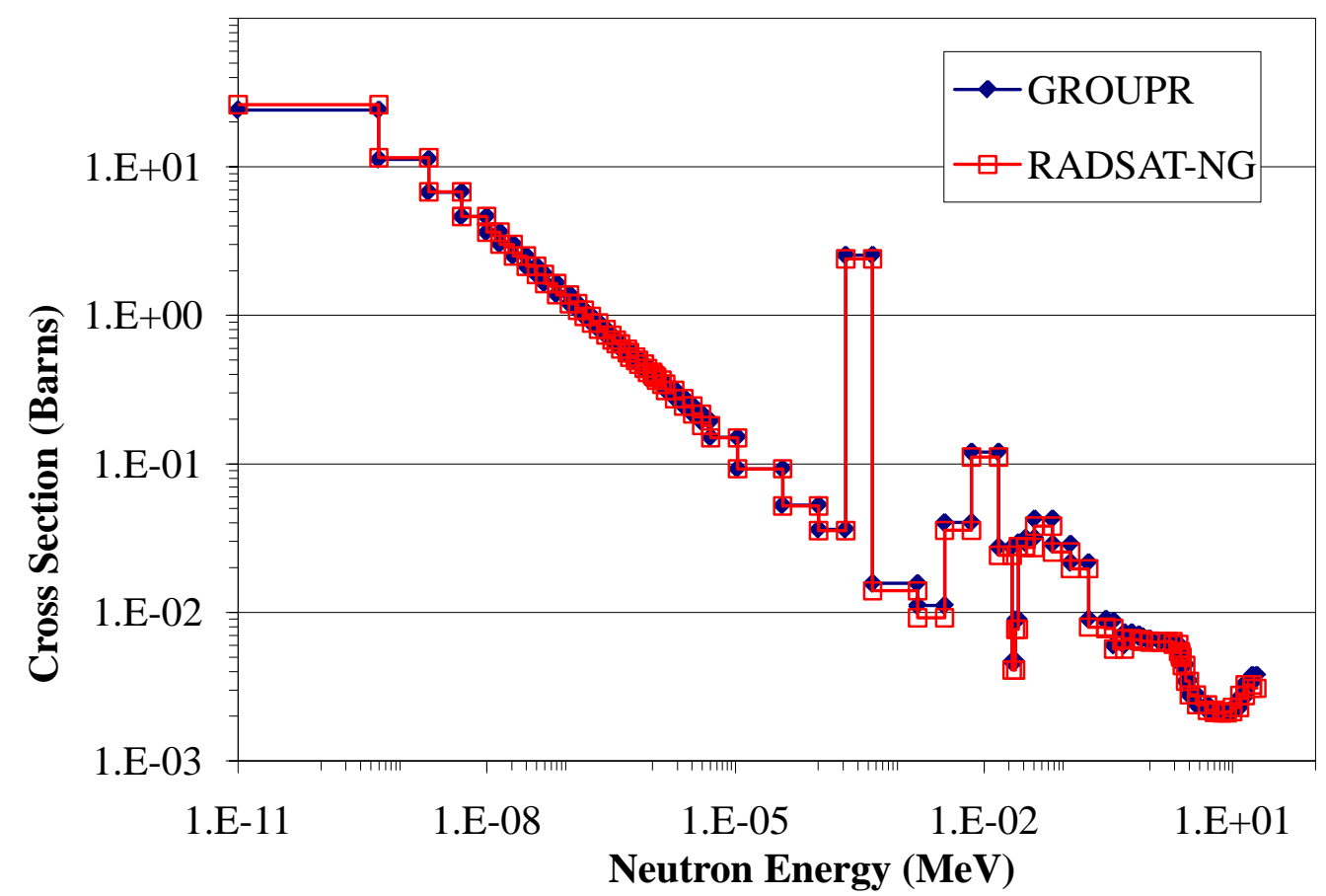

Figure 39. Comparison of the GROUPR and RADSAT-NG ${ }^{58} \mathrm{Fe}$ Radiative Capture Photon Production Cross Section 
A comparison of all of the RADSAT-NG photon production cross section for ${ }^{54} \mathrm{Fe},{ }^{56} \mathrm{Fe}$ and ${ }^{58} \mathrm{Fe}$ and the GROUPR collapsed cross sections is shown in Table 19. Most of these cross sections agree to within a few percent of the GROUPR collapsed cross sections. For the ${ }^{54} \mathrm{Fe}(\mathrm{n}, 2 \mathrm{n})$ interaction, the RADSAT-NG and GROUPR photon production cross sections differ by $22.64 \%$. The difference is due to a factor of 1.7 and a factor of 2.5 differences in the second and third highest energy groups, respectively. Similarly for the ${ }^{54} \mathrm{Fe}(\mathrm{n}, \mathrm{n} \alpha)$ photon production cross section, the RADSAT-NG and GROUPR photon production cross sections differ by $42.20 \%$ due to a difference in the second and third highest energy groups of a factor of 2.3 and 1.5, respectively. The $15 \%$ difference in the ${ }^{54} \mathrm{Fe}(\mathrm{n}, \mathrm{np})$ photon production cross section for RADSAT-NG and GROUPR is due to a factor of 1.5 difference in the fourth highest energy group. For the ${ }^{56} \mathrm{Fe}(\mathrm{n}, \mathrm{p})$ photon production cross section, the RADSAT-NG and GROUPR collapsed cross sections differ by approximately $7.16 \%$. This is due to the fact that the all of the energy groups calculated by the RADSAT-NG and GROUPR method differ consistently by a range of 6 to $15 \%$. A graphical comparison of the RADSAT-NG and GROUPR ${ }^{56} \mathrm{Fe}$ $(n, p)$ cross section is shown in Figure 40. Finally the $17.56 \%$ difference in the ${ }^{58} \mathrm{Fe}(\mathrm{n}, 2 \mathrm{n})$ photon production cross section for RADSAT-NG and GROUPR is due to a constant $13 \sim 19 \%$ difference all four energy groups the cross section is present in. The remainder of the RADSAT-NG cross sections agree with the GROUPR collapsed cross sections to within approximately $3 \%$ or better. 
Table 19. Comparison of Total Cross Section for RADSAT-NG and Cross Sections Produced by GROUPR for Iron

\begin{tabular}{|c|c|c|c|}
\hline Cross Section & $\begin{array}{l}\text { RADSAT-NG } \\
\text { Total Cross Section } \\
\text { (Barns) }\end{array}$ & $\begin{array}{l}\text { GROUPR } \\
\text { Total Cross Section } \\
\text { (Barns) }\end{array}$ & $\begin{array}{l}\text { Percent } \\
\text { Difference } \\
(\%)\end{array}$ \\
\hline${ }^{54} \mathrm{Fe}\left(\mathrm{n}, \mathrm{n}^{\prime}\right)$ & $2.09 \mathrm{E}+01$ & $2.12 \mathrm{E}+01$ & 1.22 \\
\hline${ }^{54} \mathrm{Fe}(\mathrm{n}, 2 \mathrm{n})$ & $8.22 \mathrm{E}-02$ & $6.70 \mathrm{E}-02$ & 22.64 \\
\hline${ }^{54} \mathrm{Fe}(\mathrm{n}, \mathrm{n} \alpha)$ & $6.26 \mathrm{E}-02$ & $4.40 \mathrm{E}-02$ & 42.20 \\
\hline${ }^{54} \mathrm{Fe}(\mathrm{n}, \mathrm{np})$ & $1.30 \mathrm{E}+00$ & $1.13 \mathrm{E}+00$ & 15.54 \\
\hline${ }^{54} \mathrm{Fe}(\mathrm{n}, \gamma)$ & $1.08 \mathrm{E}+02$ & $1.05 \mathrm{E}+02$ & 3.12 \\
\hline${ }^{54} \mathrm{Fe}(\mathrm{n}, \mathrm{p})$ & $7.25 \mathrm{E}+00$ & $7.34 \mathrm{E}+00$ & 1.14 \\
\hline${ }^{54} \mathrm{Fe}(\mathrm{n}, \alpha)$ & $7.99 \mathrm{E}-01$ & $7.89 \mathrm{E}-01$ & 1.27 \\
\hline${ }^{56} \mathrm{Fe}\left(\mathrm{n}, \mathrm{n}{ }^{\prime}\right)$ & $3.54 \mathrm{E}+01$ & $3.54 \mathrm{E}+01$ & $8.18 \mathrm{E}-05$ \\
\hline${ }^{56} \mathrm{Fe}(\mathrm{n}, 2 \mathrm{n})$ & $1.70 \mathrm{E}+00$ & $1.70 \mathrm{E}+00$ & 0.03 \\
\hline${ }^{56} \mathrm{Fe}(\mathrm{n}, \mathrm{n} \alpha)$ & $5.12 \mathrm{E}-02$ & $4.98 \mathrm{E}-02$ & 2.91 \\
\hline${ }^{56} \mathrm{Fe}(\mathrm{n}, \mathrm{np})$ & $4.07 \mathrm{E}-01$ & $4.04 \mathrm{E}-01$ & 0.71 \\
\hline${ }^{56} \mathrm{Fe}(\mathrm{n}, \gamma)$ & $1.38 \mathrm{E}+02$ & $1.34 \mathrm{E}+02$ & 3.03 \\
\hline${ }^{56} \mathrm{Fe}(\mathrm{n}, \mathrm{p})$ & $1.18 \mathrm{E}+00$ & $1.27 \mathrm{E}+00$ & 7.16 \\
\hline${ }^{56} \mathrm{Fe}(\mathrm{n}, \alpha)$ & $4.11 \mathrm{E}-01$ & 4.11E-01 & 0.17 \\
\hline${ }^{57} \mathrm{Fe}\left(\mathrm{n}, \mathrm{n}{ }^{\prime}\right)$ & $7.08 \mathrm{E}+01$ & ------ & $\begin{array}{l}----- \\
-1\end{array}$ \\
\hline${ }^{57} \mathrm{Fe}(\mathrm{n}, 2 \mathrm{n})$ & $7.37 \mathrm{E}+00$ & ------ & ------ \\
\hline${ }^{57} \mathrm{Fe}(\mathrm{n}, \mathrm{n} \alpha)$ & $6.98 \mathrm{E}-02$ & ------ & $\begin{array}{ll}----- \\
-1\end{array}$ \\
\hline${ }^{57} \mathrm{Fe}(\mathrm{n}, \mathrm{np})$ & $2.79 \mathrm{E}-01$ & ------ & $\begin{array}{ll}----- \\
-1\end{array}$ \\
\hline${ }^{57} \mathrm{Fe}(\mathrm{n}, \gamma)$ & $2.32 \mathrm{E}+02$ & ------ & 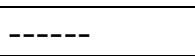 \\
\hline${ }^{57} \mathrm{Fe}(\mathrm{n}, \mathrm{p})$ & $5.24 \mathrm{E}-01$ & ------ & ------ \\
\hline${ }^{57} \mathrm{Fe}(\mathrm{n}, \alpha)$ & $3.86 \mathrm{E}-01$ & ------ & ------ \\
\hline${ }^{58} \mathrm{Fe}\left(\mathrm{n}, \mathrm{n}^{\prime}\right)$ & $3.72 \mathrm{E}+01$ & $3.71 \mathrm{E}+01$ & 0.27 \\
\hline${ }^{58} \mathrm{Fe}(\mathrm{n}, 2 \mathrm{n})$ & $6.37 \mathrm{E}+00$ & $7.73 \mathrm{E}+00$ & 17.56 \\
\hline${ }^{58} \mathrm{Fe}(\mathrm{n}, \mathrm{n} \alpha)$ & $2.59 \mathrm{E}-02$ & $2.56 \mathrm{E}-02$ & 1.15 \\
\hline${ }^{58} \mathrm{Fe}(\mathrm{n}, \mathrm{np})$ & $4.54 \mathrm{E}-02$ & $4.42 \mathrm{E}-02$ & 2.71 \\
\hline${ }^{58} \mathrm{Fe}(\mathrm{n}, \gamma)$ & $8.25 \mathrm{E}+01$ & $8.02 \mathrm{E}+01$ & 2.87 \\
\hline${ }^{58} \mathrm{Fe}(\mathrm{n}, \mathrm{p})$ & $8.81 \mathrm{E}-02$ & $8.92 \mathrm{E}-02$ & 1.25 \\
\hline${ }^{58} \mathrm{Fe}(\mathrm{n}, \alpha)$ & $1.57 \mathrm{E}-01$ & $1.58 \mathrm{E}-01$ & 0.62 \\
\hline
\end{tabular}




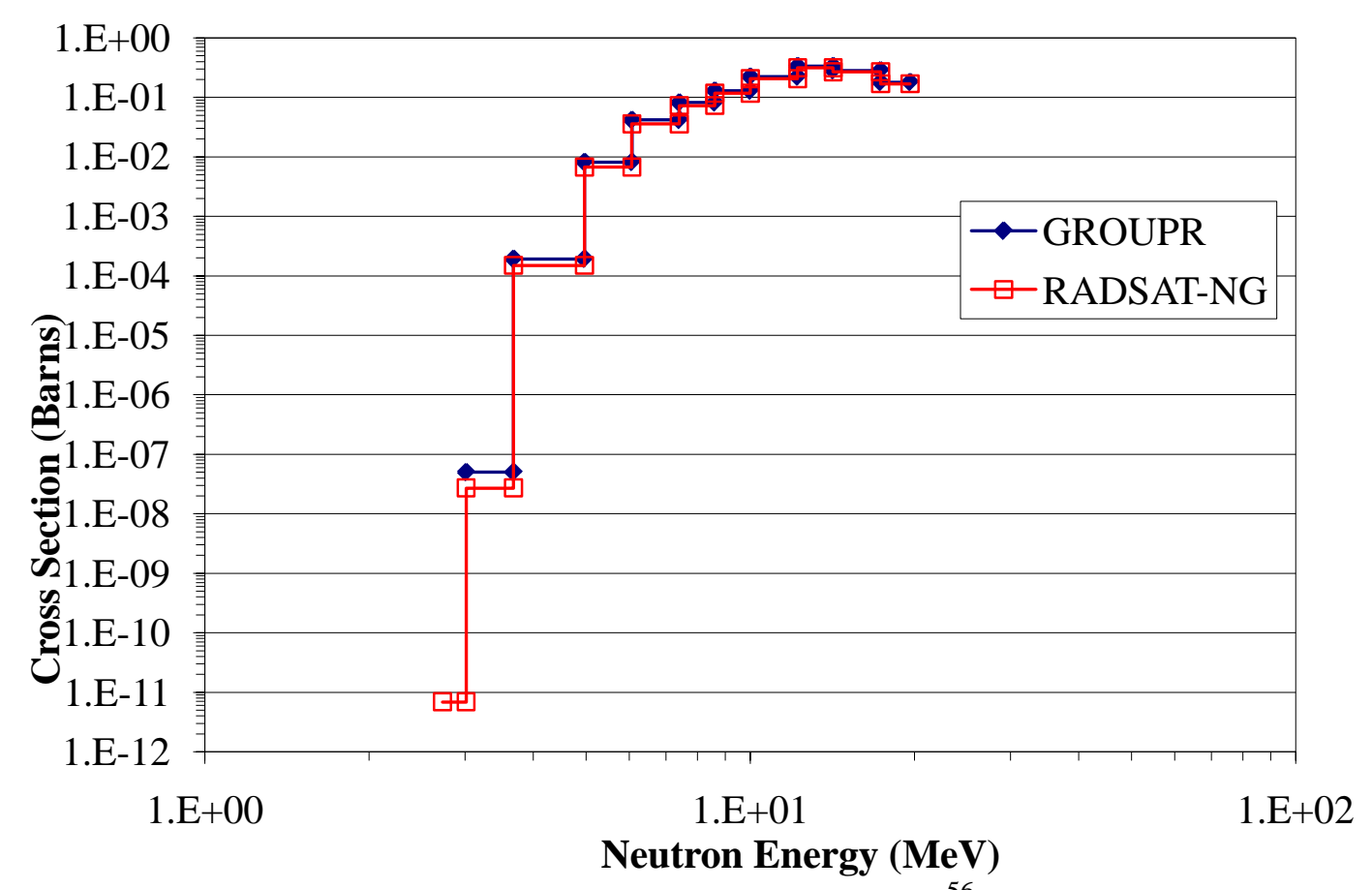

Figure 40. Comparison of the GROUPR and RADSAT-NG ${ }^{56} \mathrm{Fe}$ (n,p) Photon Production Cross Section

\subsubsection{Photon Flux within Detector}

Once the induced photon spectrum has been calculated for the irradiation of iron using the RADSAT-NG cross sections and the spatially dependent neutron flux obtained from Attila, the problem dependent photon group structure was generated using the RADSAT interface. The RADSAT group generator then was used to produce the specialized photon group structure containing 150 continuum groups and $1490.3-\mathrm{keV}$ wide peak groups, one for the $511-\mathrm{keV}$ annihilation peak and the remaining peak groups 148 most intense gamma rays produced by iron. Although a $1 / \sqrt{E}$ energy weighting factor was supplied, this will not affect the spacing of the continuum groups like it normally would since there are just enough continuum groups to place one between every peak group. Additionally, a 2-cm thick glass attenuator was applied to the source 
spectrum for the purpose of choosing the photon group structure. The purpose of the attenuator feature is to artificially reduce the intensity of the x-rays produced that would not greatly impact the detector response. This feature is only used for the purpose of choosing a photon energy bin structure. It allows the group generator to reduce the importance of these high intensity low energy gamma rays while increasing the importance of other lower intensity higher energy gamma rays which may be more noticeable in the final detector response than the x-rays.

A comparison of the volume-averaged gamma flux in the detector from the neutron irradiation of iron calculated using the RADSAT method and MCNP is shown in Figure 41. The total photon flux within the detector for the RADSAT calculation is within $7.03 \%$ of the MCNP result. This is probably due to the RADSAT calculation not properly accounting for the low angle scatter properly in this region below the $849.5 \mathrm{keV}$ gamma ray. This artifact does not typically affect the detector response because this energy region is dominated by Compton Scattering interactions in the detector. The seven most intense gamma rays for MCNP and RADSAT show typical agreement to within $2 \%$. The statistical uncertainty of the MCNP simulation for the same gamma ray emissions is equal to or greater than the percent difference between the MCNP and RADSAT calculations as shown in Table 20. 
Table 20. Comparison of Photon Flux for Top Seven Most Intense Peaks Produced by the Neutron Irradiation of Iron

\begin{tabular}{|l|l|l|l|l|}
\hline $\begin{array}{l}\text { Gamma-Ray } \\
\text { Energy (MeV) }\end{array}$ & $\begin{array}{l}\text { MCNP } \\
\left(\boldsymbol{\gamma} / \mathbf{c m}^{2} \text {-sec- }\right. \\
\text { source particle) }\end{array}$ & $\begin{array}{l}\text { MCNP Relative } \\
\text { Uncertainty (\%) }\end{array}$ & $\begin{array}{l}\text { RADSAT } \\
\left(\boldsymbol{\gamma} / \mathbf{c m}^{\mathbf{2}} \text {-sec- }\right. \\
\text { source particle) }\end{array}$ & $\begin{array}{l}\text { Percent } \\
\text { Difference } \\
(\mathbf{\%})\end{array}$ \\
\hline 0.8495 & $5.52 \mathrm{E}-06$ & 1.16 & $5.63 \mathrm{E}-06$ & 1.92 \\
\hline 1.24 & $5.89 \mathrm{E}-07$ & 3.62 & $5.91 \mathrm{E}-07$ & 0.20 \\
\hline 1.811 & $3.51 \mathrm{E}-07$ & 4.71 & $3.52 \mathrm{E}-07$ & 0.42 \\
\hline 2.113 & $2.11 \mathrm{E}-07$ & 6.13 & $2.11 \mathrm{E}-07$ & 0.41 \\
\hline 2.273 & $1.22 \mathrm{E}-07$ & 7.8 & $1.20 \mathrm{E}-07$ & 1.24 \\
\hline 2.517 & $1.02 \mathrm{E}-07$ & 9.13 & $1.03 \mathrm{E}-07$ & 0.71 \\
\hline 2.6031 & $1.15 \mathrm{E}-07$ & 10.66 & $1.15 \mathrm{E}-07$ & 0.63 \\
\hline Total & $1.29 \mathrm{E}-05$ & 0.75 & $1.38 \mathrm{E}-05$ & 7.03 \\
\hline
\end{tabular}

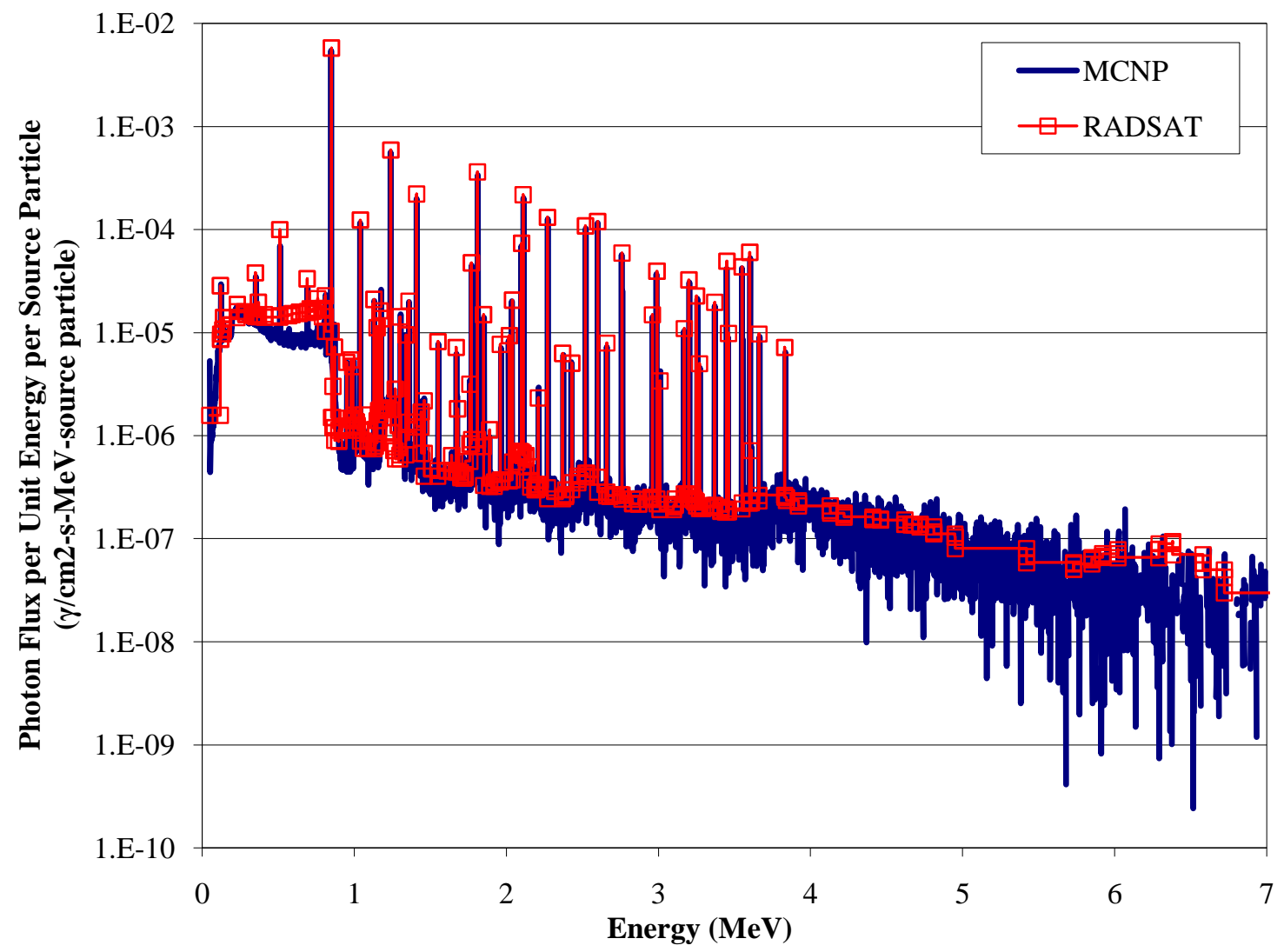

Figure 41. Comparison of Volume-Averaged Photon Flux in Detector using RADSAT and MCNP for Iron 


\section{CHAPTER 4}

\section{CONCLUSIONS}

Based on the series of simple benchmarking problems, and the comparison to MCNP5 results, the coupled Monte Carlo-deterministic transport methods being developed and implemented in RADSAT are capable of accurately and efficiently modeling neutron-induced gamma rays for high-resolution gamma-ray spectroscopy applications. The series of simple benchmark problems showed good agreement between RADSAT- and MCNP-calculated photon production rates and photon transport to a detector location, typically within $5 \%$ for the 5 highest intensity gamma rays. The polyethylene test case demonstrated good agreement between RADSAT and MCNP for a pulse-height tally, within 5\% for the prominent peak regions, in a high-resolution HPGe spectrometer.

The key enabler for RADSAT neutron-gamma method is the RADSAT-NG multigroup cross-section preparation method development in this work which, unlike currently available neutron-gamma cross-section libraries and preparation methods, tabulates the discrete-energy and continuum neutron-induced photon source terms separately. The comparison of the RADSAT-NG cross section library and cross sections collapsed using the GROUPR subroutine of NJOY typically showed good agreement. The main discrepancy present in the comparison of the two methods is in the first and last energy groups with cross sections. The general shape and magnitude of the RADSATNG multigroup cross-section preparation method is the same as the cross sections collapsed using the GROUPR subroutine of NJOY. 
While demonstrating solution accuracy is the necessary first step in the use of RADSAT for coupled neutron-photon problems for high-resolution gamma-ray spectroscopy applications, the primary motivator for using the coupled method over pure Monte Carlo method is the potential for significantly lower computational times which may enable new ways of approaching some high-resolution gamma-ray applications. In this work, the RADSAT and MCNP5 computational times for each problem were recorded, and normalized to a typical single-processor $3 \mathrm{GHz}$ desktop computer. For the series of test cases, the run times for RADSAT were approximately 25-500 times shorter than for MCNP, when assuming a $40 \mathrm{mCi}^{252} \mathrm{Cf}$ neutron source and 600 seconds of "realworld measurement time," as shown in Table 21.

Table 21. Comparison of MCNP and RADSAT Computational Times for Simple Cube Problems

\begin{tabular}{|l|c|c|c|}
\hline Simulation & $\begin{array}{l}\text { RADSAT } \\
\text { (hours) }\end{array}$ & MCNP (hours) & $\begin{array}{c}\text { Run-Time } \\
\text { Ratio }\end{array}$ \\
\hline Polyethylene & 5 & 914 & 183 \\
\hline Phosphorus & 1.9 & 910 & 479 \\
\hline Nitrogen & 3 & 84 & 28 \\
\hline Oxygen & 3.5 & 93 & 27 \\
\hline Iron & 5 & 198 & 40 \\
\hline
\end{tabular}

The run-time comparisons can vary depending on the composition of materials in the problem and size or complexity of the problem geometry. The calculations completed in RADSAT were used primarily to complete a proof of principle, so little effort was spent optimizing the RADSAT calculational parameters in order to reduce run time. Two forms of variance reduction were used in the MCNP simulations, the geometric biasing of the neutron source toward the cube and neutron importance weighting in the detector 
volume to compare the induced photon spectrum from specific materials. However, additional variance reduction techniques could substantially reduce run times for specific problems.

This study represents a proof-of-principle study for the coupled Monte Carlodeterministic method to simulate neutron-induced gamma rays for high-resolution gamma-ray spectroscopy applications. This work, therefore, represents only a preliminary assessment of RADSAT methods using a narrow subset of neutron-induced photon problems. 


\section{CHAPTER 5 \\ RECOMMENDATIONS}

Although promising results have been obtained from the proof-of-concept

analysis, additional testing to determine the range of applicability of the method will need to be completed before this method will be fully integrated into the RADSAT package. The results produced in this study were for a simple geometry and only covered a limited number of materials. Although the RADSAT-NG cross-section preparation method has been shown to produce acceptable results for the materials tested, the method still needs to be tested for more materials, particularly materials containing unresolved resonances. More complex geometries should also be tested to determine how the assumption of isotropic photon production affects the photon flux at the detector location. The systematic bias between MCNP and Attila calculated flux at low energies although not central to this work, must be resolved, and understood in order to increase confidence in the RADSAT coupled neutron gamma and neutron-only calculations. Additional evaluation of spatial discretization in the calculation of neutron flux and neutron-induced photon source terms will shed more light on both aspects-accuracy and computational efficiency — and allow a more thorough evaluation of the strengths and limitations of RADSAT methods over a wide range of radiation detection problems.

Experimental validation of computer codes is an important step in determining if the computational method is working properly. A set of benchmarking experiments should be developed to determine if the RADSAT coupled neutron gamma method is working properly. It should be noted that the ability to properly benchmark the 
RADSAT-NG method will depend on the accuracy of the underlying cross section library that was collapsed, not just the cross section collapsing method or the transport method. The experimental validation will help point out short comings that may be common to both the RADSAT-NG method and MCNP.

In order for the methods developed in this study to be properly integrated into the RADSAT interface, some additions to the RADSAT user interface are necessary. The RADSAT-NG cross-section preparation method has already been implemented into a program RADSAT-NG_Collapser which is currently being tested. The calculations of the neutron-induced photon spectra are completed using a Visual Basic script external from the RADSAT interface. The results must then be loaded into the RADSAT interface as source terms. In order to remove this intermediate step, these calculations will be completed within the RADSAT interface. The integration of these procedures into the code has not yet begun but is anticipated to begin in the near future. 


\section{APPENDIX A}

\section{Example Attila Input File for Neutron Transport Problem in $\mathrm{C}_{2} \mathrm{H}_{4}$}

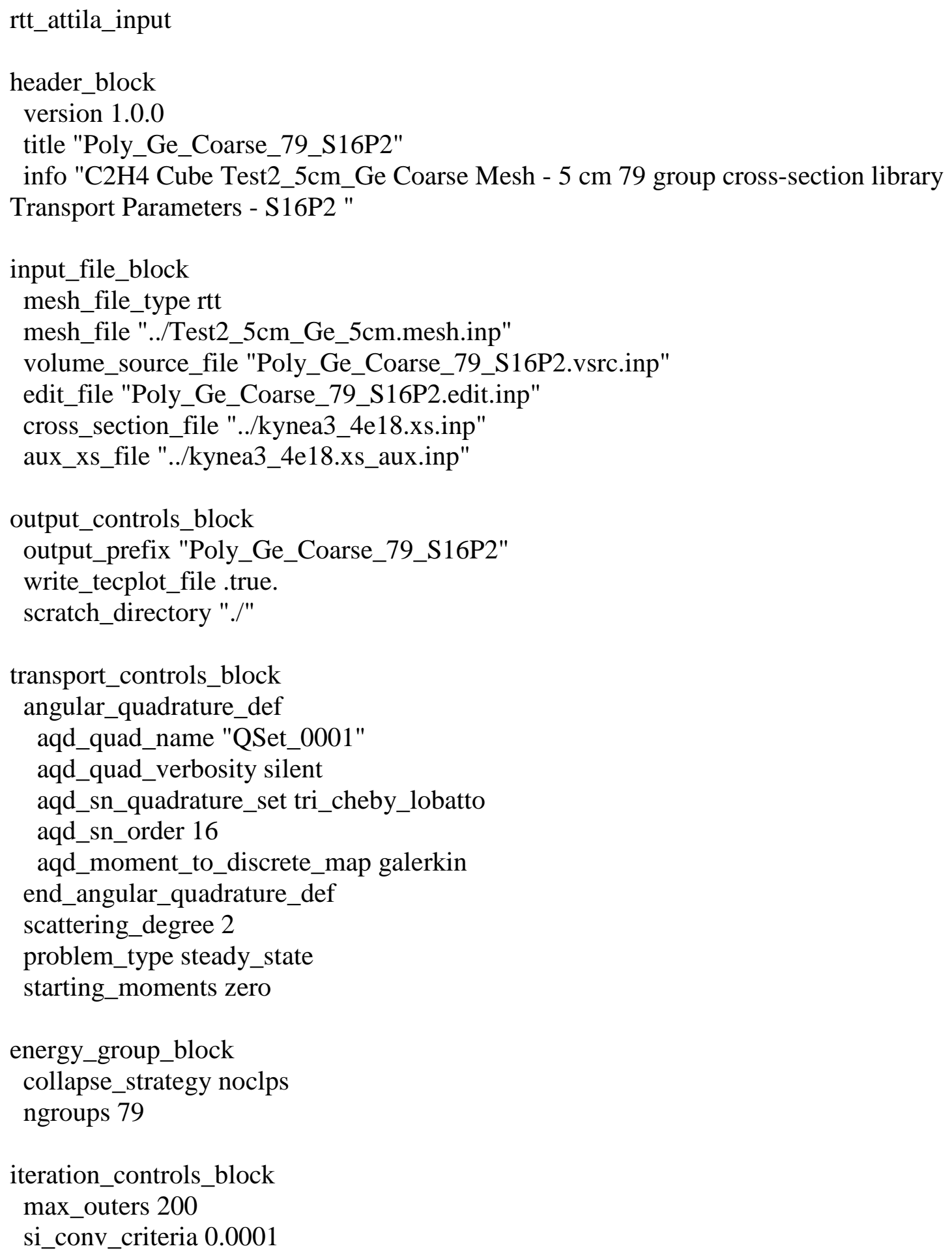


dummy_solution .false.

mesh_region_definition_block

region_index 1

side_index 2

region_def 1 "Universe" 20.001205

region_def 2 "Source_Box" 20.001205

region_def 3 "detector" 20.001205

region_def 4 "cube4_4_4" 10.5

region_def 5 "cube4_4_3" 10.5

region_def 6 "cube4_4_2" 10.5

region_def 7 "cube4_4_1" 10.5

region_def 8 "cube4_3_4" 10.5

region_def 9 "cube4_3_3" 10.5

region_def 10 "cube4_3_2" 10.5

region_def 11 "cube4_3_1" 10.5

region_def 12 "cube4_2_4" 10.5

region_def 13 "cube4_2_3" 10.5

region_def 14 "cube4_2_2" 10.5

region_def 15 "cube4_2_1" 10.5

region_def 16 "cube4_1_4" 10.5

region_def 17 "cube4_1_3" 10.5

region_def 18 "cube4_1_2" 10.5

region_def 19 "cube4_1_1" 10.5

region_def 20 "cube3_4_4" 10.5

region_def 21 "cube3_4_3" 10.5

region_def 22 "cube3_4_2" 10.5

region_def 23 "cube3_4_1" 10.5

region_def 24 "cube3_3_4" 10.5

region_def 25 "cube3_3_3" 10.5

region_def 26 "cube3_3_2" 10.5

region_def 27 "cube3_3_1" 10.5

region_def 28 "cube3_2_4" 10.5

region_def 29 "cube3_2_3" 10.5

region_def 30 "cube3_2_2" 10.5

region_def 31 "cube3_2_1" 10.5

region_def 32 "cube3_1_4" 10.5

region_def 33 "cube3_1_3" 10.5

region_def 34 "cube3_1_2" 10.5

region_def 35 "cube3_1_1" 10.5

region_def 36 "cube2_4_4" 10.5

region_def 37 "cube2_4_3" 10.5

region_def 38 "cube2_4_2" 10.5

region_def 39 "cube2_4_1" 10.5

region_def 40 "cube2_3_4" 10.5

region_def 41 "cube2_3_3" 10.5 
region_def 42 "cube2_3_2" 10.5

region_def 43 "cube2_3_1" 10.5

region_def 44 "cube2_2_4" 10.5

region_def 45 "cube2_2_3" 10.5

region_def 46 "cube2_2_2" 10.5

region_def 47 "cube2_2_1" 10.5

region_def 48 "cube2_1_4" 10.5

region_def 49 "cube2_1_3" 10.5

region_def 50 "cube2_1_2" 10.5

region_def 51 "cube2_1_1" 10.5

region_def 52 "cube1_4_4" 10.5

region_def 53 "cube1_4_3" 10.5

region_def 54 "cube1_4_2" 10.5

region_def 55 "cube1_4_1" 10.5

region_def 56 "cube1_3_4" 10.5

region_def 57 "cube1_3_3" 10.5

region_def 58 "cube1_3_2" 10.5

region_def 59 "cube1_3_1" 10.5

region_def 60 "cube1_2_4" 10.5

region_def 61 "cube1_2_3" 10.5

region_def 62 "cube1_2_2" 10.5

region_def 63 "cube1_2_1" 10.5

region_def 64 "cube1_1_4" 10.5

region_def 65 "cube1_1_3" 10.5

region_def 66 "cube1_1_2" 10.5

region_def 67 "cube1_1_1" 10.5

side_def 1 "(+)Universe@5"

side_def 79 "(+)Universe@2"

side_def 81 "(+)Universe@3"

side_def 83 "(+)Universe@4"

side_def 85 "(+)Universe@ 1"

side_def 160 "(+)Universe@6"

side_def 250 "(+)Universe/(-)cube1_4_1@1"

side_def 268 "(+)Universe/(-)cube4_4_1@3"

side_def 286 "(+)Universe/(-)cube4_4_4@3"

side_def 304 "(+)Universe/(-)cube4_1_4@2"

side_def 322 "(+)Universe/(-)cube1_4_4@2"

side_def 340 "(+)Universe/(-)cube4_4_4@1"

side_def 409 "(+)Universe/(-)detector@5"

side_def 412 "(+)Universe/(-)detector@2"

side_def 415 "(+)Universe/(-)detector@3"

side_def 418 "(+)Universe/(-)detector@4"

side_def 421 "(+)Universe/(-)detector@6"

side_def 424 "(+)Universe/(-)detector@ 1"

side_def 478 "(+)Universe/(-)Source_Box@5"

side_def 481 "(+)Universe/(-)Source_Box@2" 
side_def 484 "(+)Universe/(-)Source_Box@3" side_def 487 "(+)Universe/(-)Source_Box@4" side_def 490 "(+)Universe/(-)Source_Box@6" side_def 493 "(+)Universe/(-)Source_Box@1" side_def 1285 "(+)Universe/(-)cube4_3_4@1" side_def 1299 "(+)Universe/(-)cube4_4_3@2" side_def 1309 "(+)Universe/(-)cube4_4_4@2" side_def 1363 "(+)cube4_4_4/(-)cube3_4_4@1" side_def 1369 "(+)cube4_4_4/(-)cube4_3_4@1" side_def 1381 "(+)cube4_4_4/(-)cube4_4_3@1" side_def 1491 "(+)Universe/(-)cube4_4_3@1" side_def 1501 "(+)Universe/(-)cube4_4_2@2" side_def 1562 "(+)cube4_4_3/(-)cube3_4_3@1" side_def 1568 "(+)cube4_4_3/(-)cube4_3_3@1" side_def 1580 "(+)cube4_4_3/(-)cube4_4_2@1" side_def 1691 "(+)Universe/(-)cube4_4_2@1" side_def 1701 "(+)Universe/(-)cube4_4_1@2" side_def 1769 "(+)cube4_4_2/(-)cube3_4_2@1" side_def 1775 "(+)cube4_4_2/(-)cube4_3_2@1" side_def 1787 "(+)cube4_4_2/(-)cube4_4_1@1" side_def 1895 "(+)Universe/(-)cube4_4_1@1" side_def 1905 "(+)Universe/(-)cube4_3_4@2" side_def 1914 "(+)Universe/(-)cube4_3_1@2" side_def 1993 "(+)cube4_4_1/(-)cube3_4_1@1" side_def 1999 "(+)cube4_4_1/(-)cube4_3_1@1" side_def 2125 "(+)Universe/(-)cube4_3_3@1" side_def 2134 "(+)Universe/(-)cube4_2_4@2" side_def 2216 "(+)cube4_3_4/(-)cube3_3_4@1" side_def 2222 "(+)cube4_3_4/(-)cube4_2_4@1" side_def 2234 "(+)cube4_3_4/(-)cube4_3_3@1" side_def 2344 "(+)Universe/(-)cube4_3_2@1" side_def 2432 "(+)cube4_3_3/(-)cube3_3_3@1" side_def 2438 "(+)cube4_3_3/(-)cube4_2_3@1" side_def 2450 "(+)cube4_3_3/(-)cube4_3_2@1" side_def 2561 "(+)Universe/(-)cube4_3_1@1" side_def 2655 "(+)cube4_3_2/(-)cube3_3_2@1" side_def 2661 "(+)cube4_3_2/(-)cube4_2_2@1" side_def 2673 "(+)cube4_3_2/(-)cube4_3_1@1" side_def 2788 "(+)Universe/(-)cube4_2_1@2" side_def 2794 "(+)Universe/(-)cube4_2_4@1" side_def 2898 "(+)cube4_3_1/(-)cube3_3_1@1" side_def 2904 "(+)cube4_3_1/(-)cube4_2_1@1" side_def 3034 "(+)Universe/(-)cube4_2_3@1" side_def 3043 "(+)Universe/(-)cube4_1_4@3" side_def $3150 "(+)$ cube4_2_4/(-)cube3_2_4@1" side_def 3156 "(+)cube4_2_4/(-)cube4_1_4@1" 
side_def 3168 "(+)cube4_2_4/(-)cube4_2_3@1" side_def 3282 "(+)Universe/(-)cube4_2_2@1" side_def 3395 "(+)cube4_2_3/(-)cube3_2_3@1" side_def 3401 "(+)cube4_2_3/(-)cube4_1_3@1" side_def 3413 "(+)cube4_2_3/(-)cube4_2_2@1" side_def 3528 "(+)Universe/(-)cube4_2_1@1" side_def 3647 "(+)cube4_2_2/(-)cube3_2_2@1" side_def 3653 "(+)cube4_2_2/(-)cube4_1_2@1" side_def 3665 "(+)cube4_2_2/(-)cube4_2_1@1" side_def 3784 "(+)Universe/(-)cube4_1_1@2" side_def 3790 "(+)Universe/(-)cube4_1_1@1" side_def 3919 "(+)cube4_2_1/(-)cube3_2_1@1" side_def 3925 "(+)cube4_2_1/(-)cube4_1_1@1" side_def 4056 "(+)Universe/(-)cube4_1_4@1" side_def 4070 "(+)Universe/(-)cube4_1_3@2" side_def 4076 "(+)Universe/(-)cube3_4_4@1" side_def 4212 "(+)cube4_1_4/(-)cube3_1_4@1" side_def 4226 "(+)cube4_1_4/(-)cube4_1_3@1" side_def 4350 "(+)Universe/(-)cube4_1_3@1" side_def 4360 "(+)Universe/(-)cube4_1_2@2" side_def 4502 "(+)cube4_1_3/(-)cube3_1_3@1" side_def 4516 "(+)cube4_1_3/(-)cube4_1_2@1" side_def 4641 "(+)Universe/(-)cube4_1_2@1" side_def 4651 "(+)Universe/(-)cube4_1_1@3" side_def 4799 "(+)cube4_1_2/(-)cube3_1_2@1" side_def 4813 "(+)cube4_1_2/(-)cube4_1_1@1" side_def 4938 "(+)Universe/(-)cube3_1_4@2" side_def 4944 "(+)Universe/(-)cube3_4_1@2" side_def 5101 "(+)cube4_1_1/(-)cube3_1_1@1" side_def 5243 "(+)Universe/(-)cube3_3_4@1" side_def 5253 "(+)Universe/(-)cube3_4_4@2" side_def 5409 "(+)cube3_4_4/(-)cube2_4_4@1" side_def 5415 "(+)cube3_4_4/(-)cube3_3_4@1" side_def 5427 "(+)cube3_4_4/(-)cube3_4_3@1" side_def 5549 "(+)Universe/(-)cube3_4_3@1" side_def 5711 "(+)cube3_4_3/(-)cube2_4_3@1" side_def 5717 "(+)cube3_4_3/(-)cube3_3_3@1" side_def 5729 "(+)cube3_4_3/(-)cube3_4_2@1" side_def 5852 "(+)Universe/(-)cube3_4_2@1" side_def 6020 "(+)cube3_4_2/(-)cube2_4_2@1" side_def 6026 "(+)cube3_4_2/(-)cube3_3_2@1" side_def 6038 "(+)cube3_4_2/(-)cube3_4_1@1" side_def 6162 "(+)Universe/(-)cube3_4_1@1" side_def 6171 "(+)Universe/(-)cube3_3_1@1" side_def 6349 "(+)cube3_4_1/(-)cube2_4_1@1" side_def 6355 "(+)cube3_4_1/(-)cube3_3_1@1" 
side_def 6492 "(+)Universe/(-)cube3_2_4@1" side_def 6672 "(+)cube3_3_4/(-)cube2_3_4@1" side_def 6678 "(+)cube3_3_4/(-)cube3_2_4@1" side_def 6690 "(+)cube3_3_4/(-)cube3_3_3@1" side_def 6991 "(+)cube3_3_3/(-)cube2_3_3@1" side_def 6997 "(+)cube3_3_3/(-)cube3_2_3@1" side_def 7009 "(+)cube3_3_3/(-)cube3_3_2@1" side_def 7316 "(+)cube3_3_2/(-)cube2_3_2@1" side_def 7322 "(+)cube3_3_2/(-)cube3_2_2@1" side_def 7334 "(+)cube3_3_2/(-)cube3_3_1@1" side_def 7461 "(+)Universe/(-)cube3_2_1@1" side_def 7660 "(+)cube3_3_1/(-)cube2_3_1@1" side_def 7666 "(+)cube3_3_1/(-)cube3_2_1@1" side_def 7807 "(+)Universe/(-)cube3_1_4@1" side_def 8008 "(+)cube3_2_4/(-)cube2_2_4@1" side_def 8014 "(+)cube3_2_4/(-)cube3_1_4@1" side_def 8026 "(+)cube3_2_4/(-)cube3_2_3@1" side_def 8352 "(+)cube3_2_3/(-)cube2_2_3@1" side_def 8358 "(+)cube3_2_3/(-)cube3_1_3@1" side_def 8370 "(+)cube3_2_3/(-)cube3_2_2@1" side_def 8702 "(+)cube3_2_2/(-)cube2_2_2@1" side_def 8708 "(+)cube3_2_2/(-)cube3_1_2@1" side_def 8720 "(+)cube3_2_2/(-)cube3_2_1@1" side_def 8851 "(+)Universe/(-)cube3_1_1@2" side_def 9071 "(+)cube3_2_1/(-)cube2_2_1@1" side_def 9077 "(+)cube3_2_1/(-)cube3_1_1@1" side_def 9227 "(+)Universe/(-)cube3_1_3@1" side_def 9233 "(+)Universe/(-)cube2_4_4@1" side_def 9459 "(+)cube3_1_4/(-)cube2_1_4@1" side_def 9473 "(+)cube3_1_4/(-)cube3_1_3@1" side_def 9612 "(+)Universe/(-)cube3_1_2@1" side_def 9843 "(+)cube3_1_3/(-)cube2_1_3@1" side_def 9857 "(+)cube3_1_3/(-)cube3_1_2@1" side_def 9997 "(+)Universe/(-)cube3_1_1@1" side_def 10233 "(+)cube3_1_2/(-)cube2_1_2@1" side_def 10247 "(+)cube3_1_2/(-)cube3_1_1@1" side_def 10388 "(+)Universe/(-)cube2_4_1@2" side_def 10394 "(+)Universe/(-)cube2_1_4@2" side_def 10639 "(+)cube3_1_1/(-)cube2_1_1@1" side_def 10798 "(+)Universe/(-)cube2_4_4@2" side_def 10807 "(+)Universe/(-)cube2_3_4@1" side_def 11051 "(+)cube2_4_4/(-)cube1_4_4@1" side_def 11057 "(+)cube2_4_4/(-)cube2_3_4@1" side_def 11069 "(+)cube2_4_4/(-)cube2_4_3@1" side_def 11207 "(+)Universe/(-)cube2_4_3@1" side_def 11457 "(+)cube2_4_3/(-)cube1_4_3@1" 
side_def 11463 "(+)cube2_4_3/(-)cube2_3_3@1" side_def 11475 "(+)cube2_4_3/(-)cube2_4_2@1" side_def 11614 "(+)Universe/(-)cube2_4_2@1" side_def 11870 "(+)cube2_4_2/(-)cube1_4_2@1" side_def 11876 "(+)cube2_4_2/(-)cube2_3_2@1" side_def 11888 "(+)cube2_4_2/(-)cube2_4_1@1" side_def 12028 "(+)Universe/(-)cube2_4_1@1" side_def 12037 "(+)Universe/(-)cube2_3_1@1" side_def 12303 "(+)cube2_4_1/(-)cube1_4_1@1" side_def 12309 "(+)cube2_4_1/(-)cube2_3_1@1" side_def 12462 "(+)Universe/(-)cube2_2_4@1" side_def 12730 "(+)cube2_3_4/(-)cube1_3_4@1" side_def 12736 "(+)cube2_3_4/(-)cube2_2_4@1" side_def 12748 "(+)cube2_3_4/(-)cube2_3_3@1" side_def 13153 "(+)cube2_3_3/(-)cube1_3_3@1" side_def 13159 "(+)cube2_3_3/(-)cube2_2_3@1" side_def 13171 "(+)cube2_3_3/(-)cube2_3_2@1" side_def 13582 "(+)cube2_3_2/(-)cube1_3_2@1" side_def 13588 "(+)cube2_3_2/(-)cube2_2_2@1" side_def 13600 "(+)cube2_3_2/(-)cube2_3_1@1" side_def 13743 "(+)Universe/(-)cube2_2_1@1" side_def 14030 "(+)cube2_3_1/(-)cube1_3_1@1" side_def 14036 "(+)cube2_3_1/(-)cube2_2_1@1" side_def 14193 "(+)Universe/(-)cube2_1_4@1" side_def 14482 "(+)cube2_2_4/(-)cube1_2_4@1" side_def 14488 "(+)cube2_2_4/(-)cube2_1_4@1" side_def 14500 "(+)cube2_2_4/(-)cube2_2_3@1" side_def 14930 "(+)cube2_2_3/(-)cube1_2_3@1" side_def 14936 "(+)cube2_2_3/(-)cube2_1_3@1" side_def 14948 "(+)cube2_2_3/(-)cube2_2_2@1" side_def 15384 "(+)cube2_2_2/(-)cube1_2_2@1" side_def 15390 "(+)cube2_2_2/(-)cube2_1_2@1" side_def 15402 "(+)cube2_2_2/(-)cube2_2_1@1" side_def 15549 "(+)Universe/(-)cube2_1_1@2" side_def 15857 "(+)cube2_2_1/(-)cube1_2_1@1" side_def 15863 "(+)cube2_2_1/(-)cube2_1_1@1" side_def 16029 "(+)Universe/(-)cube2_1_3@1" side_def 16035 "(+)Universe/(-)cube1_4_4@1" side_def 16349 "(+)cube2_1_4/(-)cube1_1_4@1" side_def 16363 "(+)cube2_1_4/(-)cube2_1_3@1" side_def 16518 "(+)Universe/(-)cube2_1_2@1" side_def 16837 "(+)cube2_1_3/(-)cube1_1_3@1" side_def 16851 "(+)cube2_1_3/(-)cube2_1_2@1" side_def 17007 "(+)Universe/(-)cube2_1_1@1" side_def 17331 "(+)cube2_1_2/(-)cube1_1_2@1" side_def 17345 "(+)cube2_1_2/(-)cube2_1_1@1" 
side_def 17502 "(+)Universe/(-)cube1_4_1@3" side_def 17508 "(+)Universe/(-)cube1_1_1@3" side_def 17841 "(+)cube2_1_1/(-)cube1_1_1@1" side_def 18013 "(+)Universe/(-)cube1_4_4@3" side_def 18028 "(+)Universe/(-)cube1_4_3@2" side_def 18034 "(+)Universe/(-)cube1_3_4@1" side_def 18372 "(+)cube1_4_4/(-)cube1_3_4@1" side_def 18384 "(+)cube1_4_4/(-)cube1_4_3@1" side_def 18544 "(+)Universe/(-)cube1_4_3@1" side_def 18555 "(+)Universe/(-)cube1_4_2@2" side_def 18899 "(+)cube1_4_3/(-)cube1_3_3@1" side_def 18911 "(+)cube1_4_3/(-)cube1_4_2@1" side_def 19072 "(+)Universe/(-)cube1_4_2@1" side_def 19083 "(+)Universe/(-)cube1_4_1@2" side_def 19433 "(+)cube1_4_2/(-)cube1_3_2@1" side_def 19445 "(+)cube1_4_2/(-)cube1_4_1@1" side_def 19607 "(+)Universe/(-)cube1_3_4@2" side_def 19613 "(+)Universe/(-)cube1_3_1@2" side_def 19972 "(+)cube1_4_1/(-)cube1_3_1@1" side_def 20150 "(+)Universe/(-)cube1_3_3@1" side_def 20156 "(+)Universe/(-)cube1_2_4@1" side_def 20517 "(+)cube1_3_4/(-)cube1_2_4@1" side_def 20529 "(+)cube1_3_4/(-)cube1_3_3@1" side_def 20693 "(+)Universe/(-)cube1_3_2@1" side_def 21059 "(+)cube1_3_3/(-)cube1_2_3@1" side_def 21071 "(+)cube1_3_3/(-)cube1_3_2@1" side_def 21236 "(+)Universe/(-)cube1_3_1@1" side_def 21607 "(+)cube1_3_2/(-)cube1_2_2@1" side_def 21619 "(+)cube1_3_2/(-)cube1_3_1@1" side_def 21784 "(+)Universe/(-)cube1_2_1@2" side_def 21791 "(+)Universe/(-)cube1_2_4@2" side_def 22171 "(+)cube1_3_1/(-)cube1_2_1@1" side_def 22353 "(+)Universe/(-)cube1_2_3@1" side_def 22359 "(+)Universe/(-)cube1_1_4@1" side_def 22741 "(+)cube1_2_4/(-)cube1_1_4@1" side_def 22753 "(+)cube1_2_4/(-)cube1_2_3@1" side_def 22921 "(+)Universe/(-)cube1_2_2@1" side_def 23308 "(+)cube1_2_3/(-)cube1_1_3@1" side_def 23320 "(+)cube1_2_3/(-)cube1_2_2@1" side_def 23489 "(+)Universe/(-)cube1_2_1@1" side_def 23881 "(+)cube1_2_2/(-)cube1_1_2@1" side_def 23893 "(+)cube1_2_2/(-)cube1_2_1@1" side_def 24062 "(+)Universe/(-)cube1_1_1@1" side_def 24069 "(+)Universe/(-)cube1_1_1@2" side_def 24470 "(+)cube1_2_1/(-)cube1_1_1@1" side_def 24652 "(+)Universe/(-)cube1_1_4@3" 
side_def 24659 "(+)Universe/(-)cube1_1_4@2"

side_def 25073 "(+)cube1_1_4/(-)cube1_1_3@1"

side_def 25248 "(+)Universe/(-)cube1_1_3@1"

side_def 25255 "(+)Universe/(-)cube1_1_3@2"

side_def 25674 "(+)cube1_1_3/(-)cube1_1_2@1"

side_def 25850 "(+)Universe/(-)cube1_1_2@1"

side_def 25857 "(+)Universe/(-)cube1_1_2@2"

side_def 26281 "(+)cube1_1_2/(-)cube1_1_1@1"

material_definition_block

material_def 1 "Poly" 0

material_def 2 "Air" 0

material_comp 1160.333

material_comp 1470.667

material_comp 2610.99632

material_comp 2620.00368

cross_section_block

nparticle 1

nfine_groups 79

xsfile_format dtf

isotope_def 160 "c" 0

isotope_def 470 "h1(ch2)" 0

isotope_def 610 "n14" 0

isotope_def 620 "n15" 0

edit_xs_def 1 "absorption"

edit_xs_def 2 "nu-fission"

end_rtt_attila_input 


\section{APPENDIX B}

\section{Radion15, SCALE44 and Kynea3 Group Structure}

Table 22. Comparison of Radion15, SCALE44 and Kynea3 Neutron Energy Group Structure.

\begin{tabular}{|c|c|c|c|}
\hline Group \# & $\begin{array}{l}\text { Radion15 Group } \\
\text { Boundary (MeV) }\end{array}$ & $\begin{array}{l}\text { SCALE44 Group } \\
\text { Boundary (MeV) }\end{array}$ & $\begin{array}{l}\text { Kynea3 Group } \\
\text { Boundary }(\mathrm{MeV})\end{array}$ \\
\hline 1 & $2.00 \mathrm{E}+01-1.50 \mathrm{E}+01$ & $2.00 \mathrm{E}+01-8.19 \mathrm{E}+00$ & $1.73 \mathrm{E}+01-1.96 \mathrm{E}+01$ \\
\hline 2 & $1.50 \mathrm{E}+01-1.20 \mathrm{E}+01$ & $8.19 \mathrm{E}+00-6.43 \mathrm{E}+00$ & $1.42 \mathrm{E}+01-1.73 \mathrm{E}+01$ \\
\hline 3 & $1.20 \mathrm{E}+01-1.00 \mathrm{E}+01$ & $6.43 \mathrm{E}+00-4.80 \mathrm{E}+00$ & $1.22 \mathrm{E}+01-1.42 \mathrm{E}+01$ \\
\hline 4 & $1.00 \mathrm{E}+01-7.79 \mathrm{E}+00$ & $4.80 \mathrm{E}+00-3.00 \mathrm{E}+00$ & $1.00 \mathrm{E}+01-1.22 \mathrm{E}+01$ \\
\hline 5 & $7.79 \mathrm{E}+00-6.07 \mathrm{E}+00$ & $3.00 \mathrm{E}+00-2.48 \mathrm{E}+00$ & $8.61 \mathrm{E}+00-1.00 \mathrm{E}+01$ \\
\hline 6 & $6.07 \mathrm{E}+00-4.72 \mathrm{E}+00$ & $2.48 \mathrm{E}+00-2.35 \mathrm{E}+00$ & $7.41 \mathrm{E}+00-8.61 \mathrm{E}+00$ \\
\hline 7 & $4.72 \mathrm{E}+00-3.68 \mathrm{E}+00$ & $2.35 \mathrm{E}+00-1.85 \mathrm{E}+00$ & $6.07 \mathrm{E}+00-7.41 \mathrm{E}+00$ \\
\hline 8 & $3.68 \mathrm{E}+00-2.87 \mathrm{E}+00$ & $1.85 \mathrm{E}+00-1.40 \mathrm{E}+00$ & $4.97 \mathrm{E}+00-6.07 \mathrm{E}+00$ \\
\hline 9 & $2.87 \mathrm{E}+00-2.23 \mathrm{E}+00$ & $1.40 \mathrm{E}+00-9.00 \mathrm{E}-01$ & $3.68 \mathrm{E}+00-4.97 \mathrm{E}+00$ \\
\hline 10 & $2.23 \mathrm{E}+00-1.74 \mathrm{E}+00$ & 9.00E-01 - 4.00E-01 & $3.01 \mathrm{E}+00-3.68 \mathrm{E}+00$ \\
\hline 11 & $1.74 \mathrm{E}+00-1.19 \mathrm{E}+00$ & $4.00 \mathrm{E}-01-1.00 \mathrm{E}-01$ & $2.73 \mathrm{E}+00-3.01 \mathrm{E}+00$ \\
\hline 12 & $1.19 \mathrm{E}+00-8.21 \mathrm{E}-01$ & 1.00 E-01 - 2.50E-02 & $2.47 \mathrm{E}+00-2.73 \mathrm{E}+00$ \\
\hline 13 & 8.21E-01 - 2.35E-01 & $2.50 \mathrm{E}-02-1.70 \mathrm{E}-02$ & $2.37 \mathrm{E}+00-2.47 \mathrm{E}+00$ \\
\hline 14 & $2.35 \mathrm{E}-01-6.74 \mathrm{E}-02$ & $1.70 \mathrm{E}-02-3.00 \mathrm{E}-03$ & $2.35 \mathrm{E}+00-2.37 \mathrm{E}+00$ \\
\hline 15 & $6.74 \mathrm{E}-02-1.93 \mathrm{E}-02$ & $3.00 \mathrm{E}-03-5.50 \mathrm{E}-04$ & $2.23 \mathrm{E}+00-2.35 \mathrm{E}+00$ \\
\hline 16 & $1.93 \mathrm{E}-02-5.53 \mathrm{E}-03$ & 5.50E-04 - 1.00E-04 & $1.92 \mathrm{E}+00-2.23 \mathrm{E}+00$ \\
\hline 17 & 5.53E-03-3.54E-04 & 1.00E-04 - 3.00E-05 & $1.65 \mathrm{E}+00-1.92 \mathrm{E}+00$ \\
\hline 18 & $3.54 \mathrm{E}-04-2.26 \mathrm{E}-05$ & $3.00 \mathrm{E}-05-1.00 \mathrm{E}-05$ & $1.35 \mathrm{E}+00-1.65 \mathrm{E}+00$ \\
\hline 19 & $2.26 \mathrm{E}-05-3.47 \mathrm{E}-06$ & $1.00 \mathrm{E}-05-8.10 \mathrm{E}-06$ & $1.00 \mathrm{E}+00-1.35 \mathrm{E}+00$ \\
\hline 20 & $3.47 \mathrm{E}-06-6.25 \mathrm{E}-07$ & $8.10 \mathrm{E}-06-6.00 \mathrm{E}-06$ & $8.21 \mathrm{E}-01-1.00 \mathrm{E}+00$ \\
\hline 21 & $6.25 \mathrm{E}-07-1.24 \mathrm{E}-08$ & $6.00 \mathrm{E}-06-4.75 \mathrm{E}-06$ & 7.43E-01 - 8.21E-01 \\
\hline 22 & $1.24 \mathrm{E}-08-1.00 \mathrm{E}-11$ & $4.75 \mathrm{E}-06-3.00 \mathrm{E}-06$ & 6.08E-01 - 7.43E-01 \\
\hline 23 & ----------------------------- & $3.00 \mathrm{E}-06-1.77 \mathrm{E}-06$ & 4.98E-01 - 6.08E-01 \\
\hline 24 & ---------------------------- & 1.77E-06 - 1.00E-06 & 3.69E-01 - 4.98E-01 \\
\hline 25 & ---------------------------- & $1.00 \mathrm{E}-06-6.25 \mathrm{E}-07$ & $2.97 \mathrm{E}-01-3.69 \mathrm{E}-01$ \\
\hline 26 & --------------------------- & $6.25 \mathrm{E}-07-4.00 \mathrm{E}-07$ & 1.83E-01 - 2.97E-01 \\
\hline 27 & -------------------------- & 4.00E-07 - 3.75E-07 & $1.11 \mathrm{E}-01-1.83 \mathrm{E}-01$ \\
\hline 28 & |------------------------- & $3.75 \mathrm{E}-07-3.50 \mathrm{E}-07$ & $6.74 \mathrm{E}-02-1.11 \mathrm{E}-01$ \\
\hline 29 & ------------------------- & $3.50 \mathrm{E}-07-3.25 \mathrm{E}-07$ & 4.09E-02 - 6.74E-02 \\
\hline 30 & --------------------------- & $3.25 \mathrm{E}-07-2.75 \mathrm{E}-07$ & 3.18E-02 - 4.09E-02 \\
\hline 31 & ---------------------------- & $2.75 \mathrm{E}-07-2.50 \mathrm{E}-07$ & $2.61 \mathrm{E}-02-3.18 \mathrm{E}-02$ \\
\hline 32 & 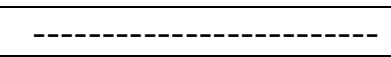 & $2.50 \mathrm{E}-07-2.25 \mathrm{E}-07$ & $2.42 \mathrm{E}-02-2.61 \mathrm{E}-02$ \\
\hline 33 & -------------------------- & $2.25 \mathrm{E}-07-2.00 \mathrm{E}-07$ & $2.19 \mathrm{E}-02-2.42 \mathrm{E}-02$ \\
\hline 34 & ------------------------- & $2.00 \mathrm{E}-07-1.50 \mathrm{E}-07$ & $1.50 \mathrm{E}-02-2.19 \mathrm{E}-02$ \\
\hline
\end{tabular}


Table 22 Cont.

\begin{tabular}{|c|c|c|c|}
\hline Group \# & $\begin{array}{l}\text { Radion15 Group } \\
\text { Boundary (MeV) }\end{array}$ & $\begin{array}{l}\text { SCALE44 Group } \\
\text { Boundary }(\mathrm{MeV})\end{array}$ & $\begin{array}{l}\text { Kynea3 Group } \\
\text { Boundary }(\mathrm{MeV})\end{array}$ \\
\hline 35 & ------------------------- & $1.50 \mathrm{E}-07-1.00 \mathrm{E}-07$ & $7.10 \mathrm{E}-03-1.50 \mathrm{E}-02$ \\
\hline 36 & ------------------------- & $1.00 \mathrm{E}-07-7.00 \mathrm{E}-08$ & $3.35 \mathrm{E}-03-7.10 \mathrm{E}-03$ \\
\hline 37 & ---------------------------- & $7.00 \mathrm{E}-08-5.00 \mathrm{E}-08$ & $1.58 \mathrm{E}-03-3.35 \mathrm{E}-03$ \\
\hline 38 & ---------------------------- & $5.00 \mathrm{E}-08-4.00 \mathrm{E}-08$ & $4.54 \mathrm{E}-04-1.58 \mathrm{E}-03$ \\
\hline 39 & 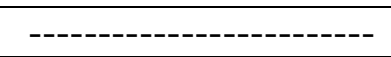 & 4.00E-08 - 3.00E-08 & $2.14 \mathrm{E}-04-4.54 \mathrm{E}-04$ \\
\hline 40 & --------------------------- & $3.00 \mathrm{E}-08-2.53 \mathrm{E}-08$ & $1.01 \mathrm{E}-04-2.14 \mathrm{E}-04$ \\
\hline 41 & 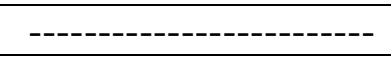 & $2.53 \mathrm{E}-08-1.00 \mathrm{E}-08$ & $3.73 \mathrm{E}-05-1.01 \mathrm{E}-04$ \\
\hline 42 & ------------------------- & $1.00 \mathrm{E}-08-7.50 \mathrm{E}-09$ & $1.07 \mathrm{E}-05-3.73 \mathrm{E}-05$ \\
\hline 43 & -------------------------- & 7.50E-09-3.00E-09 & $5.04 \mathrm{E}-06-1.07 \mathrm{E}-05$ \\
\hline 44 & ------------------------- & $3.00 \mathrm{E}-09-1.00 \mathrm{E}-11$ & $3.93 \mathrm{E}-06-5.04 \mathrm{E}-06$ \\
\hline 45 & --------------------------- & ---------------------------- & $3.06 \mathrm{E}-06-3.93 \mathrm{E}-06$ \\
\hline 46 & ---------------------------- & ---------------------------- & $2.38 \mathrm{E}-06-3.06 \mathrm{E}-06$ \\
\hline 47 & 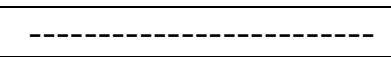 & --------------------------- & $1.86 \mathrm{E}-06-2.38 \mathrm{E}-06$ \\
\hline 48 & ------------------------- & -------------------------- & $1.45 \mathrm{E}-06-1.86 \mathrm{E}-06$ \\
\hline 49 & --------------------------- & --------------------------- & $1.30 \mathrm{E}-06-1.45 \mathrm{E}-06$ \\
\hline 50 & ------------------------- & ------------------------- & $1.13 \mathrm{E}-06-1.30 \mathrm{E}-06$ \\
\hline 51 & ------------------------- & $\begin{array}{l}----------------------- \\
\end{array}$ & $1.08 \mathrm{E}-06-1.13 \mathrm{E}-06$ \\
\hline 52 & ------------------------- & -------------------------- & $1.04 \mathrm{E}-06-1.08 \mathrm{E}-06$ \\
\hline 53 & ----------------------------- & ----------------------------- & $1.00 \mathrm{E}-06-1.04 \mathrm{E}-06$ \\
\hline 54 & ---------------------------- & ---------------------------- & $8.76 \mathrm{E}-07-1.00 \mathrm{E}-06$ \\
\hline 55 & ------------------------- & ------------------------- & $8.00 \mathrm{E}-07-8.76 \mathrm{E}-07$ \\
\hline 56 & $\begin{array}{l}\text {------------------------- } \\
\end{array}$ & $\begin{array}{l}\text {------------------------- } \\
\end{array}$ & $6.83 \mathrm{E}-07-8.00 \mathrm{E}-07$ \\
\hline 57 & ----------------------------- & ----------------------------- & $6.25 \mathrm{E}-07-6.83 \mathrm{E}-07$ \\
\hline 58 & -------------------------- & -------------------------- & $5.32 \mathrm{E}-07-6.25 \mathrm{E}-07$ \\
\hline 59 & ------------------------- & $\begin{array}{l}\text {------------------------ } \\
\end{array}$ & $5.00 \mathrm{E}-07-5.32 \mathrm{E}-07$ \\
\hline 60 & ---------------------------- & --------------------------- & $4.14 \mathrm{E}-07-5.00 \mathrm{E}-07$ \\
\hline 61 & ----------------------------- & ----------------------------- & $3.67 \mathrm{E}-07-4.14 \mathrm{E}-07$ \\
\hline 62 & ------------------------- & ------------------------- & $3.25 \mathrm{E}-07-3.67 \mathrm{E}-07$ \\
\hline 63 & -------------------------- & -------------------------- & $2.75 \mathrm{E}-07-3.25 \mathrm{E}-07$ \\
\hline 64 & --------------------------- & --------------------------- & $2.25 \mathrm{E}-07-2.75 \mathrm{E}-07$ \\
\hline 65 & ------------------------- & -------------------------- & $1.84 \mathrm{E}-07-2.25 \mathrm{E}-07$ \\
\hline 66 & ------------------------- & -------------------------- & $1.50 \mathrm{E}-07-1.84 \mathrm{E}-07$ \\
\hline 67 & ---------------------------- & --------------------------- & $1.25 \mathrm{E}-07-1.50 \mathrm{E}-07$ \\
\hline 68 & ---------------------------- & --------------------------- & $1.00 \mathrm{E}-07-1.25 \mathrm{E}-07$ \\
\hline 69 & ------------------------- & -------------------------- & $7.00 \mathrm{E}-08-1.00 \mathrm{E}-07$ \\
\hline 70 & ------------------------- & ------------------------- & $5.00 \mathrm{E}-08-7.00 \mathrm{E}-08$ \\
\hline 71 & --------------------------- & ---------------------------- & $4.00 \mathrm{E}-08-5.00 \mathrm{E}-08$ \\
\hline 72 & |-------------------------- & |-------------------------- & $3.00 \mathrm{E}-08-4.00 \mathrm{E}-08$ \\
\hline 73 & ------------------------- & -------------------------- & $2.10 \mathrm{E}-08-3.00 \mathrm{E}-08$ \\
\hline 74 & ------------------------- & -------------------------- & $1.45 \mathrm{E}-08-2.10 \mathrm{E}-08$ \\
\hline 75 & 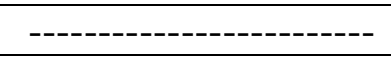 & 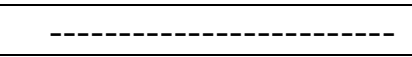 & $1.00 \mathrm{E}-08-1.45 \mathrm{E}-08$ \\
\hline 76 & ------------------------- & -------------------------- & $5.00 \mathrm{E}-09-1.00 \mathrm{E}-08$ \\
\hline
\end{tabular}


Table 22 Cont.

\begin{tabular}{|c|c|c|c|}
\hline Group \# & $\begin{array}{l}\text { Radion15 Group } \\
\text { Boundary (MeV) }\end{array}$ & $\begin{array}{l}\text { SCALE44 Group } \\
\text { Boundary (MeV) }\end{array}$ & $\begin{array}{l}\text { Kynea3 Group } \\
\text { Boundary }(\mathrm{MeV})\end{array}$ \\
\hline 77 & -------------------------- & -------------------------- & $2.00 \mathrm{E}-09-5.00 \mathrm{E}-09$ \\
\hline 78 & --------------------------- & 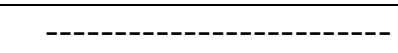 & $5.00 \mathrm{E}-10-2.00 \mathrm{E}-09$ \\
\hline 79 & 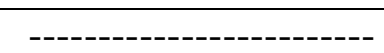 & --------------------------- & $1.00 \mathrm{E}-11-5.00 \mathrm{E}-10$ \\
\hline
\end{tabular}




\section{APPENDIX C}

\section{MCNP5 Input File for $\mathrm{C}_{2} \mathrm{H}_{4}$ Cube}

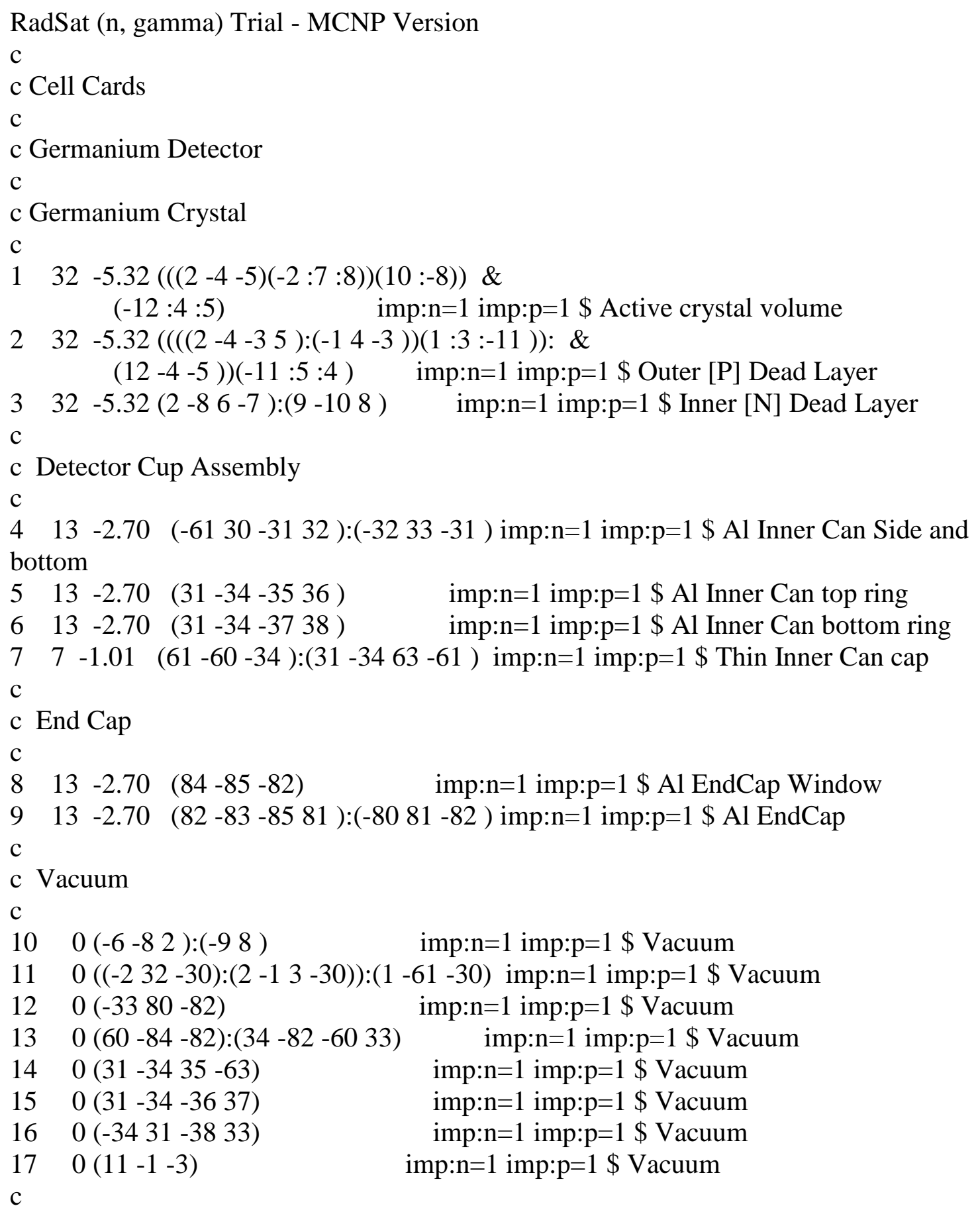




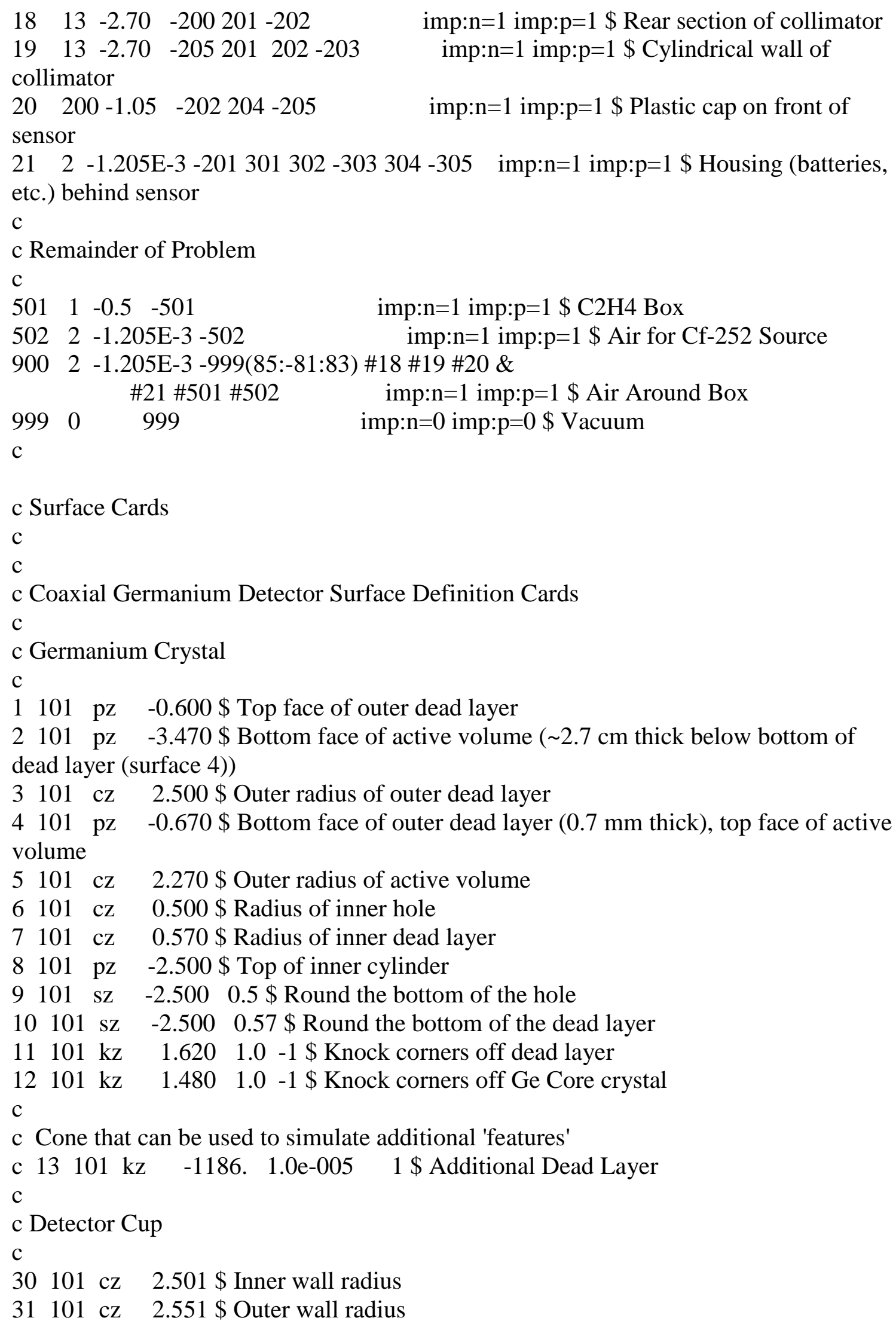


$32101 \mathrm{pz}-4.800$ \$ Inside [top] of bottom piece

$33101 \mathrm{pz}-5.000$ \$ Outside [bottom] of bottom piece

$34101 \mathrm{cz} \quad 2.650$ \$ Outer screw Flange radius

$35101 \mathrm{pz}-1.590$ \$ Top of top screw Flange

$36101 \mathrm{pz}-2.090$ \$ Bottom of top screw flange

$37101 \mathrm{pz}-2.580$ \$ Top of bottom screw Flange

$38101 \mathrm{pz}-3.080$ \$ Bottom of bottom screw flange

$\mathrm{c}$

c Cap for Detector Cup

$\mathrm{c}$

$60101 \mathrm{pz} \quad-0.570$ \$ Outer face

$61101 \mathrm{pz} \quad-0.599$ \$ Inner face, top of detector cup

$63101 \mathrm{pz}-0.900$ \$ Lower extent of cap

$\mathrm{c}$

c End Cap and End Cap Window

c

$80101 \mathrm{pz} \quad-7.000$ \$ Inside Lower bound of detector end cap

$81101 \mathrm{pz} \quad-7.100$ \$ Outside Lower bound of detector end cap

$82101 \mathrm{cz} \quad 3.150$ \$ Inside radius of end cap

$83101 \mathrm{cz} \quad 3.250$ \$ Outside radius of end cap

$84101 \mathrm{pz} \quad-0.100$ \$ Inside detector Face (Window)

$85101 \mathrm{pz} \quad 0.000$ \$ Outside detector face (Window)

$\mathrm{c}$

c Collimator around detector crystal

$200101 \mathrm{pz}-7.1$ \$ front face of rear section

$201101 \mathrm{pz}-7.4$ \$ back face of rear section

$202101 \mathrm{cz} \quad 3.25$ \$ inside of collimator wall

$203101 \mathrm{cz} \quad 3.55$ \$ outside of collimator wall

$204101 \mathrm{pz} \quad 0.0$ \$ front surface of collimator wall

$205101 \mathrm{pz} \quad 0.2$ \$ front face of front section

$\mathrm{c}$

c Remainder of Problem

$\mathrm{c}$

501 rpp -10 10 -10 10-10 10 \$ C2H4 Box

$\mathrm{c}$

502 box $-15-0.25-0.25$

$\begin{array}{lll}-0.5 & 0 & 0\end{array}$

$\begin{array}{lll}0 & 0.5 & 0\end{array}$

$\begin{array}{llll}0 & 0 & 0.5 & \$ \mathrm{Cf}-252 \text { Source }\end{array}$

c

999 rpp -16.5 31 -11.5 11.5 -11.5 11.5 \$ Problem Boundary

$\mathrm{C}$

c $* * * * * * * * * * * * * * * * * * * * * * * * * * * * * * * *$ Data cards

**************************************

$\mathrm{c}$ 


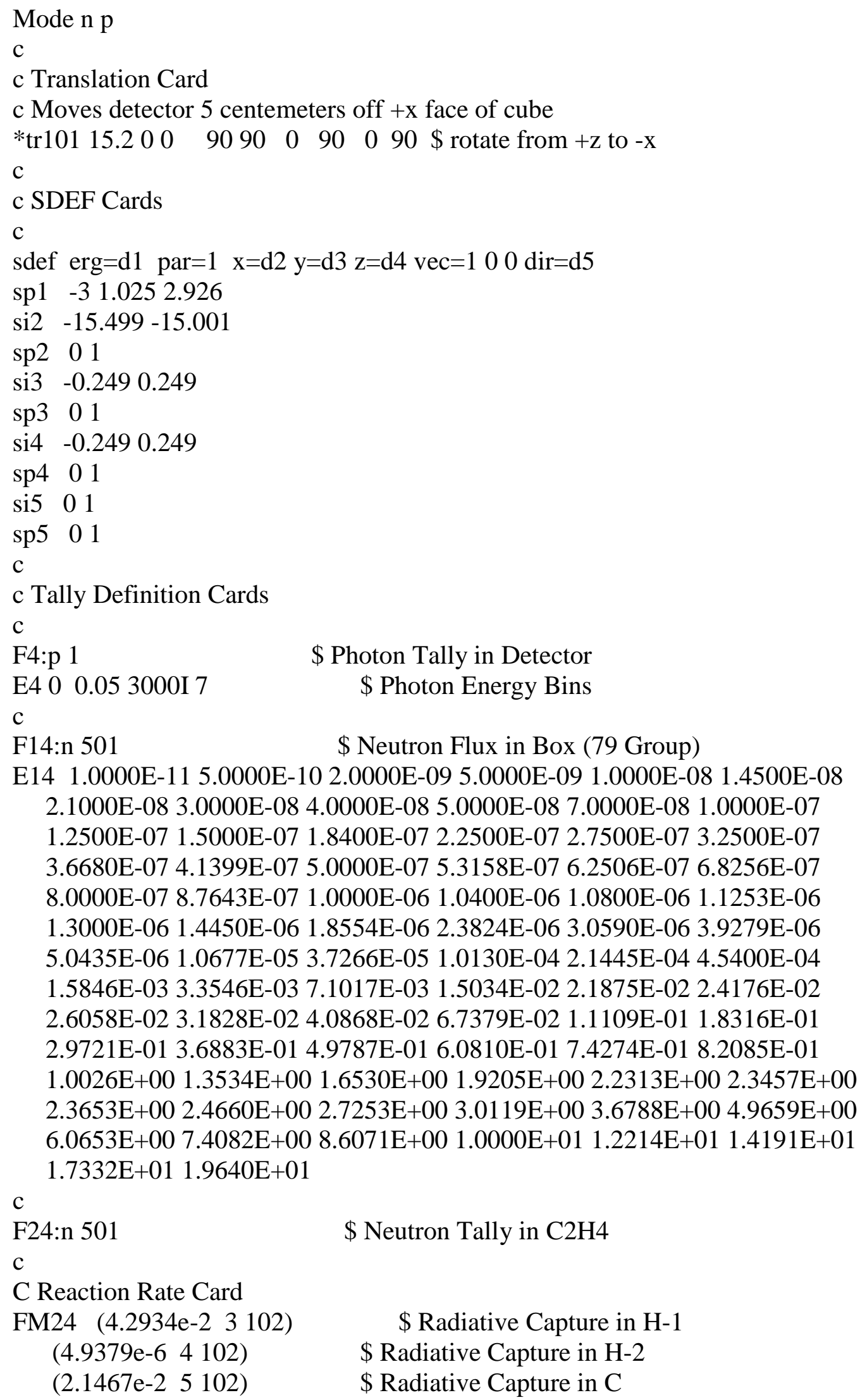

C Reaction Rate Card FM24 (4.2934e-2 3 102)

\$ Radiative Capture in $\mathrm{C}$ 


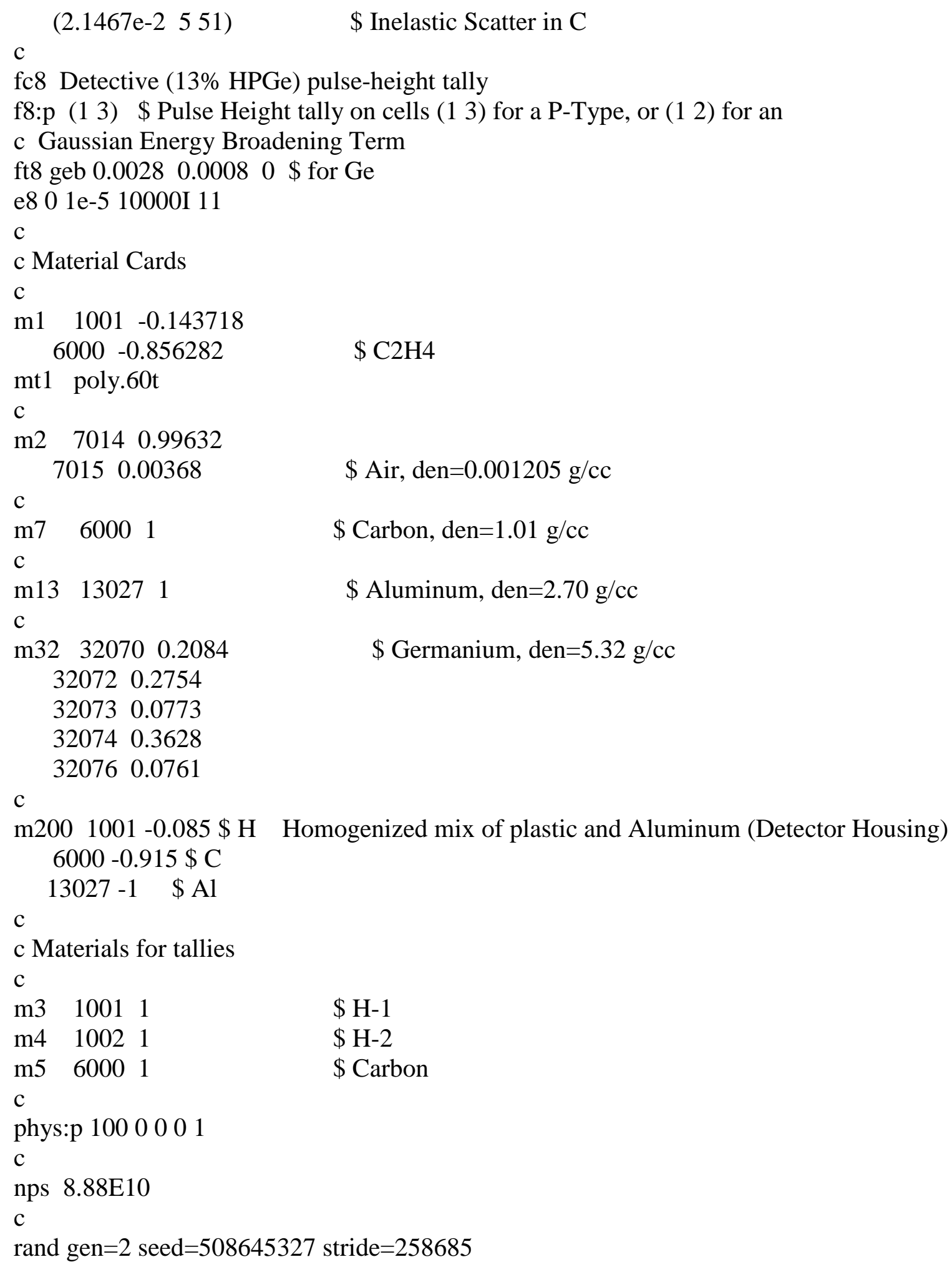




\section{APPENDIX D \\ Summary of NJOY99.259 Modules Used in Calculations}

The MODER module is purely a formatting routine that is used within NJOY to convert files to binary and vice versa [3]. The MODER module was used to convert the ENDF file into the binary format used by NJOY for computation procedures within the code.

RECONR module was used to reconstruct pointwise cross-sections from the resonance parameters and interpolation schemes in the ENDF/B format. The RECONR module in NJOY produces pointwise cross sections on a union energy grid that is suitable for linear interpolation in the PENDF format [3]. Additionally the summation cross sections, such as the total and inelastic, "are reconstructed to be exactly equal to the sum of their parts at all energies.

BROADR module was used to calculate the Doppler broadening of the pointwise PENDF cross sections. The method employed by the BROADR module is often referred to as "kernel broadening" since "it uses a detailed integration of the integral equation defining the effective cross section. It is a fully accurate method, treating all resonance and nonresonance cross sections including multilevel effects." [3]

UNRESR module was used to produce the effective self-shielded pointwise cross section in the unresolved resonance range using the resonance parameters in the ENDF files. Although none of the elements included in this project include unresolved resonances, it does not hurt to include this subroutine in the input file. Since there are no unresolved resonance parameters in the ENDF files, the subroutine does not perform a 
function but acts as a place holder for future NJOY runs which could contain unresolved resonance features.

ACER module was used to produce a point-wise cross-section file (of the form used by MCNP5). The ACER module was key to the process because the file it produces contains separate cross-sections for the production of discrete gamma rays and for continuum photons. Both are provided on a single union energy grid suitable for linear interpolation, making subsequent collapsing relatively straightforward. No data points were removed using the ACER subroutine. It was used merely as a formatting routine to convert the PENDF file, an internal file used by NJOY, to a file more easily collapsed.

GROUPR module was used to produce photon production matrices from ENDF/B-VI data. The Bondarenko narrow-resonance flux weighting approximation [3] was used for all calculations. 


\section{APPENDIX E}

\section{NJOY99.259 Input File to Produce ACER file for Hydrogen}

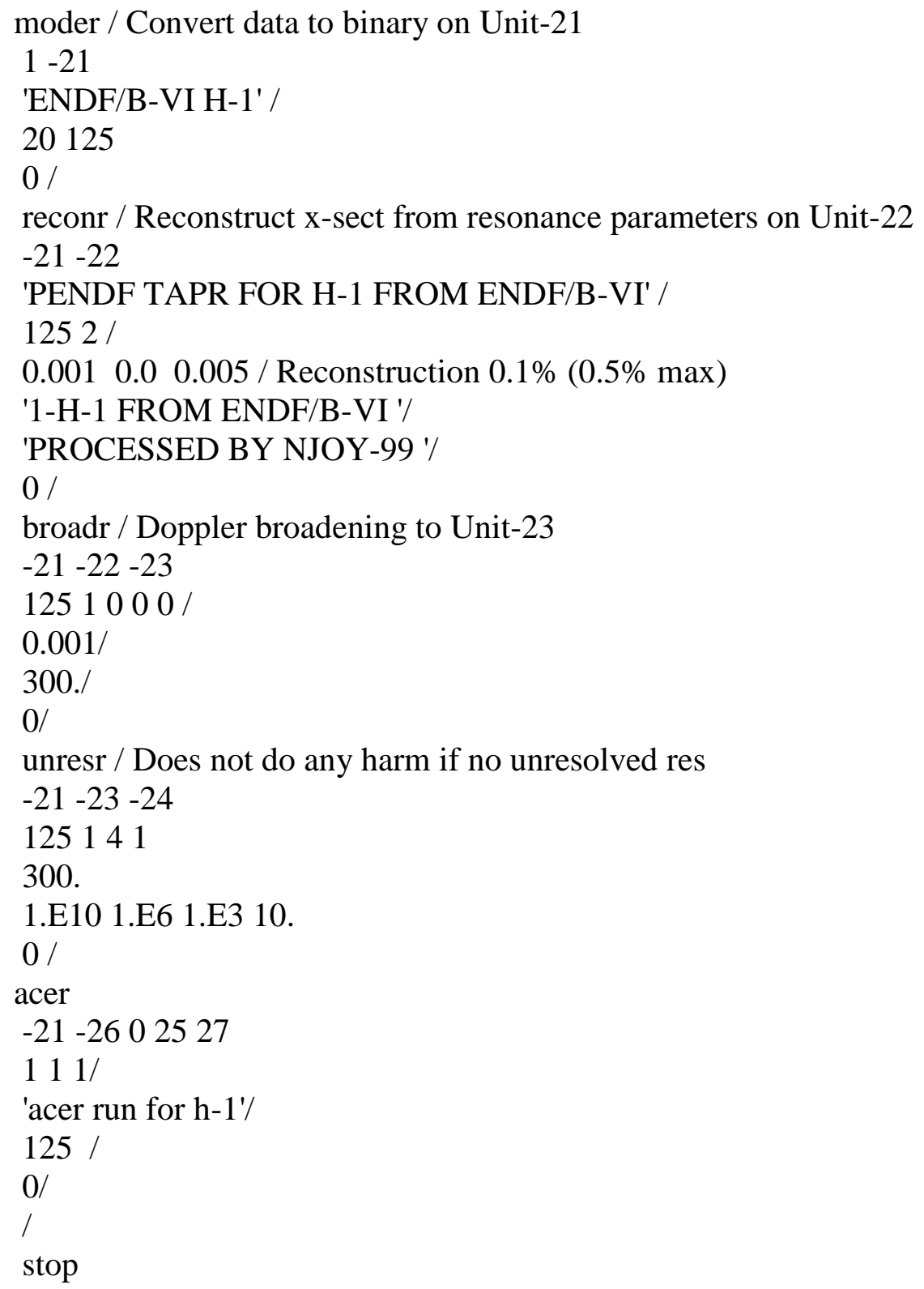




\section{APPENDIX F}

\section{RADSAT-NG Cross Section Example Format}

\section{ZAID}

\begin{tabular}{|c|c|c|c|c|c|}
\hline \multicolumn{6}{|c|}{$\mathrm{E}_{\gamma 1}(\mathrm{MeV})$} \\
\hline$\sigma_{\mathrm{g} 1}$ & $\sigma_{\mathrm{g} 2}$ & $\sigma_{\mathrm{g} 3}$ & $\sigma_{\mathrm{g} 4}$ & $\sigma_{\mathrm{g} 5}$ & $\sigma_{g 6}$ \\
\hline$\sigma_{\mathrm{g} 7}$ & $\sigma_{\mathrm{g} 8}$ & $\sigma_{\mathrm{g} 9}$ & $\sigma_{\mathrm{g} 10}$ & $\sigma_{\mathrm{g} 11}$ & $\sigma_{\mathrm{g} 12}$ \\
\hline g13 & $\sigma_{\mathrm{g} 14}$ & $\sigma_{\mathrm{g} 15}$ & $\sigma_{\mathrm{g} 16}$ & $\sigma_{\mathrm{g} 17}$ & $\sigma_{\mathrm{g} 18}$ \\
\hline$\sigma_{\mathrm{g} 19}$ & $\sigma_{\mathrm{g} 20}$ & $\sigma_{\mathrm{g} 21}$ & $\sigma_{\mathrm{g} 22}$ & $\sigma_{\mathrm{g} 23}$ & $\sigma_{\mathrm{g} 24}$ \\
\hline$\sigma_{\mathrm{g} 25}$ & $\sigma_{\mathrm{g} 26}$ & $\sigma_{\mathrm{g} 27}$ & $\sigma_{\mathrm{g} 28}$ & $\sigma_{\mathrm{g} 29}$ & $\sigma_{\mathrm{g} 30}$ \\
\hline$\sigma_{\mathrm{g} 31}$ & $\sigma_{\mathrm{g} 32}$ & $\sigma_{\mathrm{g} 33}$ & $\sigma_{\mathrm{g} 34}$ & $\sigma_{\mathrm{g} 35}$ & $\sigma_{\mathrm{g} 36}$ \\
\hline$\sigma_{\mathrm{g} 37}$ & $\sigma_{\mathrm{g} 38}$ & $\sigma_{\mathrm{g} 39}$ & $\sigma_{\mathrm{g} 40}$ & $\sigma_{\mathrm{g} 41}$ & $\sigma_{\mathrm{g} 42}$ \\
\hline$\sigma_{\mathrm{g} 43}$ & $\sigma_{\mathrm{g} 44}$ & $\sigma_{\mathrm{g} 45}$ & $\sigma_{\mathrm{g} 46}$ & $\sigma_{\mathrm{g} 47}$ & $\sigma_{\mathrm{g} 48}$ \\
\hline$\sigma_{\mathrm{g} 49}$ & $\sigma_{\mathrm{g} 50}$ & $\sigma_{\mathrm{g} 51}$ & $\sigma_{\mathrm{g} 52}$ & $\sigma_{\mathrm{g} 53}$ & $\sigma_{\mathrm{g} 54}$ \\
\hline$\sigma_{\mathrm{g} 55}$ & $\sigma_{\mathrm{g} 56}$ & $\sigma_{\mathrm{g} 57}$ & $\sigma_{\mathrm{g} 58}$ & $\sigma_{\mathrm{g} 59}$ & $\sigma_{\mathrm{g} 60}$ \\
\hline$\sigma_{\mathrm{g} 61}$ & $\sigma_{\mathrm{g} 62}$ & $\sigma_{\mathrm{g} 63}$ & $\sigma_{\mathrm{g} 64}$ & $\sigma_{\mathrm{g} 65}$ & $\sigma_{\mathrm{g} 66}$ \\
\hline$\sigma_{\mathrm{g} 67}$ & $\sigma_{\mathrm{g} 68}$ & $\sigma_{\mathrm{g} 69}$ & $\sigma_{\mathrm{g} 70}$ & $\sigma_{\mathrm{g} 71}$ & $\sigma_{\mathrm{g} 72}$ \\
\hline$\sigma_{\mathrm{g} 73}$ & $\sigma_{\mathrm{g} 74}$ & $\sigma_{\mathrm{g} 75}$ & $\sigma_{\mathrm{g} 76}$ & $\sigma_{\mathrm{g} 77}$ & $\sigma_{\mathrm{g} 78}$ \\
\hline \multicolumn{6}{|l|}{$\sigma_{\mathrm{g} 79}$} \\
\hline \multicolumn{6}{|c|}{$\mathrm{E}_{\gamma 2}(\mathrm{MeV})$} \\
\hline$\sigma_{\mathrm{g} 1}$ & $\sigma_{\mathrm{g} 2}$ & $\sigma_{\mathrm{g} 3}$ & $\sigma_{\mathrm{g} 4}$ & $\sigma_{\mathrm{g} 5}$ & $\sigma_{\mathrm{g} 6}$ \\
\hline$\sigma_{\mathrm{g} 7}$ & $\sigma_{\mathrm{g} 8}$ & $\sigma_{\mathrm{g} 9}$ & $\sigma_{\mathrm{g} 10}$ & $\sigma_{\mathrm{g} 11}$ & $\sigma_{\mathrm{g} 12}$ \\
\hline$\sigma_{\mathrm{g} 13}$ & $\sigma_{\mathrm{g} 14}$ & $\sigma_{\mathrm{g} 15}$ & $\sigma_{\mathrm{g} 16}$ & $\sigma_{\mathrm{g} 17}$ & $\sigma_{\mathrm{g} 18}$ \\
\hline$\sigma_{\mathrm{g} 19}$ & $\sigma_{\mathrm{g} 20}$ & $\sigma_{\mathrm{g} 21}$ & $\sigma_{\mathrm{g} 22}$ & $\sigma_{\mathrm{g} 23}$ & $\sigma_{\mathrm{g} 24}$ \\
\hline$\sigma_{\mathrm{g} 25}$ & $\sigma_{\mathrm{g} 26}$ & $\sigma_{\mathrm{g} 27}$ & $\sigma_{\mathrm{g} 28}$ & $\sigma_{\mathrm{g} 29}$ & $\sigma_{\mathrm{g} 30}$ \\
\hline$\sigma_{\mathrm{g} 31}$ & $\sigma_{\mathrm{g} 32}$ & $\sigma_{\mathrm{g} 33}$ & $\sigma_{\mathrm{g} 34}$ & $\sigma_{\mathrm{g} 35}$ & $\sigma_{\mathrm{g} 36}$ \\
\hline$\sigma_{\mathrm{g} 37}$ & $\sigma_{\mathrm{g} 38}$ & $\sigma_{\mathrm{g} 39}$ & $\sigma_{\mathrm{g} 40}$ & $\sigma_{\mathrm{g} 41}$ & $\sigma_{\mathrm{g} 42}$ \\
\hline$\sigma_{\mathrm{g} 43}$ & $\sigma_{\mathrm{g} 44}$ & $\sigma_{\mathrm{g} 45}$ & $\sigma_{\mathrm{g} 46}$ & $\sigma_{\mathrm{g} 47}$ & $\sigma_{\mathrm{g} 48}$ \\
\hline$\sigma_{\mathrm{g} 49}$ & $\sigma_{\mathrm{g} 50}$ & $\sigma_{\mathrm{g} 51}$ & $\sigma_{\mathrm{g} 52}$ & $\sigma_{\mathrm{g} 53}$ & $\sigma_{\mathrm{g} 54}$ \\
\hline$\sigma_{\mathrm{g} 55}$ & $\sigma_{\mathrm{g} 56}$ & $\sigma_{\mathrm{g} 57}$ & $\sigma_{\mathrm{g} 58}$ & $\sigma_{\mathrm{g} 59}$ & $\sigma_{\mathrm{g} 60}$ \\
\hline$\sigma_{\mathrm{g} 61}$ & $\sigma_{\mathrm{g} 62}$ & $\sigma_{\mathrm{g} 63}$ & $\sigma_{\mathrm{g} 64}$ & $\sigma_{\mathrm{g} 65}$ & $\sigma_{\mathrm{g} 66}$ \\
\hline$\sigma_{\mathrm{g} 67}$ & $\sigma_{\mathrm{g} 68}$ & $\sigma_{\mathrm{g} 69}$ & $\sigma_{\mathrm{g} 70}$ & $\sigma_{\mathrm{g} 71}$ & $\sigma_{\mathrm{g} 72}$ \\
\hline$\sigma_{\mathrm{g} 73}$ & $\sigma_{\mathrm{g} 74}$ & $\sigma_{\mathrm{g} 75}$ & $\sigma_{\mathrm{g} 76}$ & $\sigma_{\mathrm{g} 77}$ & $\sigma_{\mathrm{g} 78}$ \\
\hline \multicolumn{6}{|l|}{$\sigma_{\mathrm{g} 79}$} \\
\hline \multicolumn{6}{|c|}{$\mathrm{E}_{\gamma 3}(\mathrm{MeV})$} \\
\hline$\sigma_{\mathrm{g} 1}$ & $\sigma_{\mathrm{g} 2}$ & $\sigma_{\mathrm{g} 3}$ & $\sigma_{\mathrm{g} 4}$ & $\sigma_{\mathrm{g} 5}$ & $\sigma_{g 6}$ \\
\hline$\sigma_{\mathrm{g} 7}$ & $\sigma_{\mathrm{g} 8}$ & $\sigma_{\mathrm{g} 9}$ & $\sigma_{\mathrm{g} 10}$ & $\sigma_{\mathrm{g} 11}$ & $\sigma_{\mathrm{g} 12}$ \\
\hline$\sigma_{\mathrm{g} 13}$ & $\sigma_{\mathrm{g} 14}$ & $\sigma_{\mathrm{g} 15}$ & $\sigma_{\mathrm{g} 16}$ & $\sigma_{\mathrm{g} 17}$ & $\sigma_{\mathrm{g} 18}$ \\
\hline
\end{tabular}




$\begin{array}{llllll}\sigma_{\mathrm{g} 19} & \sigma_{\mathrm{g} 20} & \sigma_{\mathrm{g} 21} & \sigma_{\mathrm{g} 22} & \sigma_{\mathrm{g} 23} & \sigma_{\mathrm{g} 24} \\ \sigma_{\mathrm{g} 25} & \sigma_{\mathrm{g} 26} & \sigma_{\mathrm{g} 27} & \sigma_{\mathrm{g} 28} & \sigma_{\mathrm{g} 29} & \sigma_{\mathrm{g} 30} \\ \sigma_{\mathrm{g} 31} & \sigma_{\mathrm{g} 32} & \sigma_{\mathrm{g} 33} & \sigma_{\mathrm{g} 34} & \sigma_{\mathrm{g} 35} & \sigma_{\mathrm{g} 36} \\ \sigma_{\mathrm{g} 37} & \sigma_{\mathrm{g} 38} & \sigma_{\mathrm{g} 39} & \sigma_{\mathrm{g} 40} & \sigma_{\mathrm{g} 41} & \sigma_{\mathrm{g} 42} \\ \sigma_{\mathrm{g} 43} & \sigma_{\mathrm{g} 44} & \sigma_{\mathrm{g} 45} & \sigma_{\mathrm{g} 46} & \sigma_{\mathrm{g} 47} & \sigma_{\mathrm{g} 48} \\ \sigma_{\mathrm{g} 49} & \sigma_{\mathrm{g} 50} & \sigma_{\mathrm{g} 51} & \sigma_{\mathrm{g} 52} & \sigma_{\mathrm{g} 53} & \sigma_{\mathrm{g} 54} \\ \sigma_{\mathrm{g} 55} & \sigma_{\mathrm{g} 56} & \sigma_{\mathrm{g} 57} & \sigma_{\mathrm{g} 58} & \sigma_{\mathrm{g} 59} & \sigma_{\mathrm{g} 60} \\ \sigma_{\mathrm{g} 61} & \sigma_{\mathrm{g} 62} & \sigma_{\mathrm{g} 63} & \sigma_{\mathrm{g} 64} & \sigma_{\mathrm{g} 65} & \sigma_{\mathrm{g} 66} \\ \sigma_{\mathrm{g} 67} & \sigma_{\mathrm{g} 68} & \sigma_{\mathrm{g} 69} & \sigma_{\mathrm{g} 70} & \sigma_{\mathrm{g} 71} & \sigma_{\mathrm{g} 72} \\ \sigma_{\mathrm{g} 73} & \sigma_{\mathrm{g} 74} & \sigma_{\mathrm{g} 75} & \sigma_{\mathrm{g} 76} & \sigma_{\mathrm{g} 77} & \sigma_{\mathrm{g} 78} \\ \sigma_{\mathrm{g} 79} & & & & & \end{array}$

Continuum $* * * * * * * * * * * * * * * * * * * *$

\section{Continuum}

\begin{tabular}{|c|c|c|c|c|c|}
\hline$\sigma_{\mathrm{g} 1 \rightarrow \mathrm{p} 1}$ & $\sigma_{\mathrm{g} 2 \rightarrow \mathrm{p} 1}$ & $\sigma_{\mathrm{g} 3 \rightarrow \mathrm{p} 1}$ & $\sigma_{\mathrm{g} 4 \rightarrow \mathrm{p} 1}$ & $\sigma_{\mathrm{g} 5 \rightarrow \mathrm{p} 1}$ & $\sigma_{\mathrm{g} 6 \rightarrow \mathrm{p} 1}$ \\
\hline$\sigma_{\mathrm{g} 7 \rightarrow \mathrm{p} 1}$ & $\sigma_{\mathrm{g} 8 \rightarrow \mathrm{p} 1}$ & $\sigma_{\mathrm{g} 9 \rightarrow \mathrm{p} 1}$ & $\sigma_{\mathrm{g} 10 \rightarrow \mathrm{p} 1}$ & $\sigma_{\mathrm{g} 11 \rightarrow \mathrm{p} 1}$ & $\sigma_{\mathrm{g} 12 \rightarrow \mathrm{p} 1}$ \\
\hline$\sigma_{\mathrm{g} 13 \rightarrow \mathrm{p} 1}$ & $\sigma_{\mathrm{g} 14 \rightarrow \mathrm{p} 1}$ & $\sigma_{\mathrm{g} 15 \rightarrow \mathrm{p} 1}$ & $\sigma_{\mathrm{g} 16 \rightarrow \mathrm{p} 1}$ & $\sigma_{\mathrm{g} 17 \rightarrow \mathrm{p} 1}$ & $\sigma_{\mathrm{g} 18 \rightarrow \mathrm{p} 1}$ \\
\hline$\sigma_{\mathrm{g} 19 \rightarrow \mathrm{p} 1}$ & $\sigma_{\mathrm{g} 20 \rightarrow \mathrm{p} 1}$ & $\sigma_{\mathrm{g} 21 \rightarrow \mathrm{p} 1}$ & $\sigma_{\mathrm{g} 22 \rightarrow \mathrm{p} 1}$ & $\sigma_{\mathrm{g} 23 \rightarrow \mathrm{p} 1}$ & $\sigma_{\mathrm{g} 24 \rightarrow \mathrm{p} 1}$ \\
\hline$\sigma_{\mathrm{g} 25 \rightarrow \mathrm{p} 1}$ & $\sigma_{\mathrm{g} 26} \rightarrow \mathrm{p} 1$ & $\sigma_{\mathrm{g} 27 \rightarrow \mathrm{p} 1}$ & $\sigma_{\mathrm{g} 28 \rightarrow \mathrm{p} 1}$ & $\sigma_{\mathrm{g} 29 \rightarrow \mathrm{p} 1}$ & $\sigma_{\mathrm{g} 30 \rightarrow \mathrm{p} 1}$ \\
\hline$\sigma_{\mathrm{g} 31 \rightarrow \mathrm{p} 1}$ & $\sigma_{\mathrm{g} 32 \rightarrow \mathrm{p} 1}$ & $\sigma_{\mathrm{g} 33 \rightarrow \mathrm{p} 1}$ & $\sigma_{\mathrm{g} 34 \rightarrow \mathrm{p} 1}$ & $\sigma_{\mathrm{g} 35 \rightarrow \mathrm{p} 1}$ & $\sigma_{\mathrm{g} 36 \rightarrow \mathrm{p} 1}$ \\
\hline$\sigma_{\mathrm{g} 37 \rightarrow \mathrm{p} 1}$ & $\sigma_{\mathrm{g} 38 \rightarrow \mathrm{p} 1}$ & $\sigma_{\mathrm{g} 39 \rightarrow \mathrm{p} 1}$ & $\sigma_{\mathrm{g} 40 \rightarrow \mathrm{p} 1}$ & $\sigma_{\mathrm{g} 41 \rightarrow \mathrm{p} 1}$ & $\sigma_{\mathrm{g} 42 \rightarrow \mathrm{p} 1}$ \\
\hline$\sigma_{\mathrm{g} 43 \rightarrow \mathrm{p} 1}$ & $\sigma_{\mathrm{g} 44 \rightarrow \mathrm{p} 1}$ & $\sigma_{\mathrm{g} 45 \rightarrow \mathrm{p} 1}$ & $\sigma_{\mathrm{g} 46 \rightarrow \mathrm{p} 1}$ & $\sigma_{\mathrm{g} 47 \rightarrow \mathrm{p} 1}$ & $\sigma_{\mathrm{g} 48 \rightarrow \mathrm{p} 1}$ \\
\hline$\sigma_{\mathrm{g} 49 \rightarrow \mathrm{p} 1}$ & $\sigma_{\mathrm{g} 50 \rightarrow \mathrm{p} 1}$ & $\sigma_{\mathrm{g} 51 \rightarrow \mathrm{p} 1}$ & $\sigma_{\mathrm{g} 52 \rightarrow \mathrm{p} 1}$ & $\sigma_{\mathrm{g} 53 \rightarrow \mathrm{p} 1}$ & $\sigma_{\mathrm{g} 54 \rightarrow \mathrm{p} 1}$ \\
\hline$\sigma_{\mathrm{g} 55 \rightarrow \mathrm{p} 1}$ & $\sigma_{\mathrm{g} 56 \rightarrow \mathrm{p} 1}$ & $\sigma_{\mathrm{g} 57 \rightarrow \mathrm{p} 1}$ & $\sigma_{\mathrm{g} 58 \rightarrow \mathrm{p} 1}$ & $\sigma_{\mathrm{g} 59 \rightarrow \mathrm{p} 1}$ & $\sigma_{\mathrm{g} 60 \rightarrow \mathrm{p} 1}$ \\
\hline$\sigma_{\mathrm{g} 61 \rightarrow \mathrm{p} 1}$ & $\sigma_{\mathrm{g} 62 \rightarrow \mathrm{p} 1}$ & $\sigma_{\mathrm{g} 63 \rightarrow \mathrm{p} 1}$ & $\sigma_{\mathrm{g} 64 \rightarrow \mathrm{p} 1}$ & $\sigma_{\mathrm{g} 65 \rightarrow \mathrm{p} 1}$ & $\sigma_{\mathrm{g} 66 \rightarrow \mathrm{p} 1}$ \\
\hline$\sigma_{\mathrm{g} 67 \rightarrow \mathrm{p} 1}$ & $\sigma_{\mathrm{g} 68 \rightarrow \mathrm{p} 1}$ & $\sigma_{\mathrm{g} 69 \rightarrow \mathrm{p} 1}$ & $\sigma_{\mathrm{g} 70 \rightarrow \mathrm{p} 1}$ & $\sigma_{\mathrm{g} 71 \rightarrow \mathrm{p} 1}$ & $\sigma_{\mathrm{g} 72 \rightarrow \mathrm{p} 1}$ \\
\hline$\sigma_{\mathrm{g} 73 \rightarrow \mathrm{p} 1}$ & $\sigma_{\mathrm{g} 74 \rightarrow \mathrm{p} 1}$ & $\sigma_{\mathrm{g} 75 \rightarrow \mathrm{p} 1}$ & $\sigma_{\mathrm{g} 76 \rightarrow \mathrm{p} 1}$ & $\sigma_{\mathrm{g} 77 \rightarrow \mathrm{p} 1}$ & $\sigma_{\mathrm{g} 78 \rightarrow \mathrm{p} 1}$ \\
\hline$\sigma_{\mathrm{g} 79 \rightarrow \mathrm{p} 1}$ & $\sigma_{\mathrm{g} 1 \rightarrow \mathrm{p} 2}$ & $\sigma_{\mathrm{g} 2 \rightarrow \mathrm{p} 2}$ & $\sigma_{\mathrm{g} 3 \rightarrow \mathrm{p} 2}$ & $\sigma_{\mathrm{g} 4 \rightarrow \mathrm{p} 2}$ & $\sigma_{\mathrm{g} 5 \rightarrow \mathrm{p} 2}$ \\
\hline$\sigma_{\mathrm{g} 6 \rightarrow \mathrm{p} 2}$ & $\sigma_{\mathrm{g} 7 \rightarrow \mathrm{p} 2}$ & $\sigma_{\mathrm{g} 8 \rightarrow \mathrm{p} 2}$ & $\sigma_{\mathrm{g} 9 \rightarrow \mathrm{p} 2}$ & $\sigma_{\mathrm{g} 10 \rightarrow \mathrm{p} 2}$ & $\sigma_{\mathrm{g} 11 \rightarrow \mathrm{p} 2}$ \\
\hline$\sigma_{\mathrm{g} 12 \rightarrow \mathrm{p} 2}$ & $\sigma_{\mathrm{g} 13 \rightarrow \mathrm{p} 2}$ & $\sigma_{\mathrm{g} 14 \rightarrow \mathrm{p} 2}$ & $\sigma_{\mathrm{g} 15 \rightarrow \mathrm{p} 2}$ & $\sigma_{\mathrm{g} 16 \rightarrow \mathrm{p} 2}$ & $\sigma_{\mathrm{g} 17 \rightarrow \mathrm{p} 2}$ \\
\hline$\sigma_{\mathrm{g} 18 \rightarrow \mathrm{p} 2}$ & $\sigma_{\mathrm{g} 19 \rightarrow \mathrm{p} 2}$ & $\sigma_{\mathrm{g} 20 \rightarrow \mathrm{p} 2}$ & $\sigma_{\mathrm{g} 21 \rightarrow \mathrm{p} 2}$ & $\sigma_{\mathrm{g} 22 \rightarrow \mathrm{p} 2}$ & $\sigma_{\mathrm{g} 23 \rightarrow \mathrm{p} 2}$ \\
\hline$\sigma_{\mathrm{g} 24 \rightarrow \mathrm{p} 2}$ & $\sigma_{\mathrm{g} 25 \rightarrow \mathrm{p} 2}$ & $\sigma_{\mathrm{g} 26 \rightarrow \mathrm{p} 2}$ & $\sigma_{\mathrm{g} 27 \rightarrow \mathrm{p} 2}$ & $\sigma_{\mathrm{g} 28 \rightarrow \mathrm{p} 2}$ & $\sigma_{\mathrm{g} 29 \rightarrow \mathrm{p} 2}$ \\
\hline$\sigma_{\mathrm{g} 30 \rightarrow \mathrm{p} 2}$ & $\sigma_{\mathrm{g} 31 \rightarrow \mathrm{p} 2}$ & $\sigma_{\mathrm{g} 32 \rightarrow \mathrm{p} 2}$ & $\sigma_{\mathrm{g} 33 \rightarrow \mathrm{p} 2}$ & $\sigma_{\mathrm{g} 34 \rightarrow \mathrm{p} 2}$ & $\sigma_{\mathrm{g} 35 \rightarrow \mathrm{p} 2}$ \\
\hline$\sigma_{\mathrm{g} 36 \rightarrow \mathrm{p} 2}$ & $\sigma_{\mathrm{g} 37 \rightarrow \mathrm{p} 2}$ & $\sigma_{\mathrm{g} 38 \rightarrow \mathrm{p} 2}$ & $\sigma_{\mathrm{g} 39 \rightarrow \mathrm{p} 2}$ & $\sigma_{\mathrm{g} 40 \rightarrow \mathrm{p} 2}$ & $\sigma_{\mathrm{g} 41 \rightarrow \mathrm{p} 2}$ \\
\hline$\sigma_{\mathrm{g} 42 \rightarrow \mathrm{p} 2}$ & $\sigma_{\mathrm{g} 43 \rightarrow \mathrm{p} 2}$ & $\sigma_{\mathrm{g} 44 \rightarrow \mathrm{p} 2}$ & $\sigma_{\mathrm{g} 45 \rightarrow \mathrm{p} 2}$ & $\sigma_{\mathrm{g} 46 \rightarrow \mathrm{p} 2}$ & $\sigma_{\mathrm{g} 47 \rightarrow \mathrm{p} 2}$ \\
\hline$\sigma_{\mathrm{g} 48 \rightarrow \mathrm{p} 2}$ & $\sigma_{\mathrm{g} 49 \rightarrow \mathrm{p} 2}$ & $\sigma_{\mathrm{g} 50 \rightarrow \mathrm{p} 2}$ & $\sigma_{\mathrm{g} 51 \rightarrow \mathrm{p} 2}$ & $\sigma_{\mathrm{g} 52 \rightarrow \mathrm{p} 2}$ & $\sigma_{\mathrm{g} 53 \rightarrow \mathrm{p} 2}$ \\
\hline$\sigma_{\mathrm{g} 54 \rightarrow \mathrm{p} 2}$ & $\sigma_{\mathrm{g} 55 \rightarrow \mathrm{p} 2}$ & $\sigma_{\mathrm{g} 56 \rightarrow \mathrm{p} 2}$ & $\sigma_{\mathrm{g} 57 \rightarrow \mathrm{p} 2}$ & $\sigma_{\mathrm{g} 58 \rightarrow \mathrm{p} 2}$ & $\sigma_{\mathrm{g} 59 \rightarrow \mathrm{p} 2}$ \\
\hline$\sigma_{\mathrm{g} 60 \rightarrow \mathrm{p} 2}$ & $\sigma_{\mathrm{g} 61 \rightarrow \mathrm{p} 2}$ & $\sigma_{\mathrm{g} 62 \rightarrow \mathrm{p} 2}$ & $\sigma_{\mathrm{g} 63 \rightarrow \mathrm{p} 2}$ & $\sigma_{\mathrm{g} 64 \rightarrow \mathrm{p} 2}$ & $\sigma_{\mathrm{g} 65 \rightarrow \mathrm{p} 2}$ \\
\hline$\sigma_{\mathrm{g} 66 \rightarrow \mathrm{p} 2}$ & $\sigma_{\mathrm{g} 67 \rightarrow \mathrm{p} 2}$ & $\sigma_{\mathrm{g} 68 \rightarrow \mathrm{p} 2}$ & $\sigma_{\mathrm{g} 69 \rightarrow \mathrm{p} 2}$ & $\sigma_{\mathrm{g} 70 \rightarrow \mathrm{p} 2}$ & $\sigma_{\mathrm{g} 71 \rightarrow \mathrm{p} 2}$ \\
\hline$\sigma_{\mathrm{g} 72 \rightarrow \mathrm{p} 2}$ & $\sigma_{\mathrm{g} 73 \rightarrow \mathrm{p} 2}$ & $\sigma_{\mathrm{g} 74 \rightarrow \mathrm{p} 2}$ & $\sigma_{\mathrm{g} 75 \rightarrow \mathrm{p} 2}$ & $\sigma_{\mathrm{g} 76 \rightarrow \mathrm{p} 2}$ & $\sigma_{\mathrm{g} 77 \rightarrow \mathrm{p} 2}$ \\
\hline$\sigma_{\mathrm{g} 78 \rightarrow \mathrm{p} 2}$ & $\sigma_{\mathrm{g} 79 \rightarrow \mathrm{p} 2}$ & $\sigma_{\mathrm{g} 1 \rightarrow \mathrm{pn}}$ & $\sigma_{\mathrm{g} 2 \rightarrow \mathrm{pn}}$ & $\sigma_{\mathrm{g} 3 \rightarrow \mathrm{pn}}$ & $\sigma_{\mathrm{g} 4 \rightarrow \mathrm{pn}}$ \\
\hline$\sigma_{\mathrm{g} 5 \rightarrow \mathrm{pn}}$ & $\sigma_{\mathrm{g} 6 \rightarrow \mathrm{pn}}$ & $\sigma_{\mathrm{g} 7 \rightarrow \mathrm{pn}}$ & $\sigma_{\mathrm{g} 8 \rightarrow \mathrm{pn}}$ & $\sigma_{\mathrm{g} 9 \rightarrow \mathrm{pn}}$ & $\sigma_{\mathrm{g} 10 \rightarrow \mathrm{pn}}$ \\
\hline$\sigma_{\mathrm{g} 11 \rightarrow \mathrm{pn}}$ & $\sigma_{\mathrm{g} 12 \rightarrow \mathrm{pn}}$ & $\sigma_{\mathrm{g} 13 \rightarrow \mathrm{pn}}$ & $\sigma_{\mathrm{g} 14 \rightarrow \mathrm{pn}}$ & $\sigma_{\mathrm{g} 15 \rightarrow \mathrm{pn}}$ & $\sigma_{\mathrm{g} 16 \rightarrow \mathrm{pn}}$ \\
\hline$\sigma_{\mathrm{g} 17 \rightarrow \mathrm{pn}}$ & $\sigma_{\mathrm{g} 18 \rightarrow \mathrm{pn}}$ & $\sigma_{\mathrm{g} 19 \rightarrow \mathrm{pn}}$ & $\sigma_{\mathrm{g} 20 \rightarrow \mathrm{pn}}$ & $\sigma_{\mathrm{g} 21 \rightarrow \mathrm{pn}}$ & $\sigma_{\mathrm{g} 22 \rightarrow \mathrm{pn}}$ \\
\hline$\sigma_{\mathrm{g} 23 \rightarrow \mathrm{pn}}$ & $\sigma_{\mathrm{g} 24 \rightarrow \mathrm{pn}}$ & $\sigma_{\mathrm{g} 25 \rightarrow \mathrm{pn}}$ & $\sigma_{\mathrm{g} 26 \rightarrow \mathrm{pn}}$ & $\sigma_{\mathrm{g} 27 \rightarrow \mathrm{pn}}$ & $\sigma_{\mathrm{g} 28 \rightarrow \mathrm{f}}$ \\
\hline
\end{tabular}




$\begin{array}{llllll}\sigma_{\mathrm{g} 29 \rightarrow \mathrm{pn}} & \sigma_{\mathrm{g} 30 \rightarrow \mathrm{pn}} & \sigma_{\mathrm{g} 31 \rightarrow \mathrm{pn}} & \sigma_{\mathrm{g} 32 \rightarrow \mathrm{pn}} & \sigma_{\mathrm{g} 33 \rightarrow \mathrm{pn}} & \sigma_{\mathrm{g} 34 \rightarrow \mathrm{pn}} \\ \sigma_{\mathrm{g} 35 \rightarrow \mathrm{pn}} & \sigma_{\mathrm{g} 36 \rightarrow \mathrm{pn}} & \sigma_{\mathrm{g} 37 \rightarrow \mathrm{pn}} & \sigma_{\mathrm{g} 38 \rightarrow \mathrm{pn}} & \sigma_{\mathrm{g} 39 \rightarrow \mathrm{pn}} & \sigma_{\mathrm{g} 40 \rightarrow \mathrm{pn}} \\ \sigma_{\mathrm{g} 41 \rightarrow \mathrm{pn}} & \sigma_{\mathrm{g} 42 \rightarrow \mathrm{pn}} & \sigma_{\mathrm{g} 43 \rightarrow \mathrm{pn}} & \sigma_{\mathrm{g} 44 \rightarrow \mathrm{pn}} & \sigma_{\mathrm{g} 45 \rightarrow \mathrm{pn}} & \sigma_{\mathrm{g} 46 \rightarrow \mathrm{pn}} \\ \sigma_{\mathrm{g} 47 \rightarrow \mathrm{pn}} & \sigma_{\mathrm{g} 48 \rightarrow \mathrm{pn}} & \sigma_{\mathrm{g} 49 \rightarrow \mathrm{pn}} & \sigma_{\mathrm{g} 50 \rightarrow \mathrm{pn}} & \sigma_{\mathrm{g} 51 \rightarrow \mathrm{pn}} & \sigma_{\mathrm{g} 52 \rightarrow \mathrm{pn}} \\ \sigma_{\mathrm{g} 53 \rightarrow \mathrm{pn}} & \sigma_{\mathrm{g} 54 \rightarrow \mathrm{pn}} & \sigma_{\mathrm{g} 55 \rightarrow \mathrm{pn}} & \sigma_{\mathrm{g} 56 \rightarrow \mathrm{pn}} & \sigma_{\mathrm{g} 57 \rightarrow \mathrm{pn}} & \sigma_{\mathrm{g} 58 \rightarrow \mathrm{pn}} \\ \sigma_{\mathrm{g} 59 \rightarrow \mathrm{pn}} & \sigma_{\mathrm{g} 60 \rightarrow \mathrm{pn}} & \sigma_{\mathrm{g} 61 \rightarrow \mathrm{pn}} & \sigma_{\mathrm{g} 62 \rightarrow \mathrm{pn}} & \sigma_{\mathrm{g} 63 \rightarrow \mathrm{pn}} & \sigma_{\mathrm{g} 64 \rightarrow \mathrm{pn}} \\ \sigma_{\mathrm{g} 65 \rightarrow \mathrm{pn}} & \sigma_{\mathrm{g} 66 \rightarrow \mathrm{pn}} & \sigma_{\mathrm{g} 67 \rightarrow \mathrm{pn}} & \sigma_{\mathrm{g} 68 \rightarrow \mathrm{pn}} & \sigma_{\mathrm{g} 69 \rightarrow \mathrm{pn}} & \sigma_{\mathrm{g} 70 \rightarrow \mathrm{pn}} \\ \sigma_{\mathrm{g} 71 \rightarrow \mathrm{pn}} & \sigma_{\mathrm{g} 72 \rightarrow \mathrm{pn}} & \sigma_{\mathrm{g} 73 \rightarrow \mathrm{pn}} & \sigma_{\mathrm{g} 74 \rightarrow \mathrm{pn}} & \sigma_{\mathrm{g} 75 \rightarrow \mathrm{pn}} & \sigma_{\mathrm{g} 76 \rightarrow \mathrm{pn}} \\ \sigma_{\mathrm{g} 77 \rightarrow \mathrm{pn}} & \sigma_{\mathrm{g} 78 \rightarrow \mathrm{pn}} & \sigma_{\mathrm{g} 79 \rightarrow \mathrm{pn}} & & & \end{array}$




\section{APPENDIX G}

\section{NJOY99.259 Input File to Produce GROUPR file for Hydrogen}

moder / Convert data to binary on Unit-21

$1-21$

'ENDF/B-VI H-1' /

20125

0 /

reconr / Reconstruct X-sect from resonance parameters on Unit-22

$-21-22$

'PENDF TAPR FOR H-1 FROM ENDF/B-VI' /

$1252 /$

$0.0010 .00 .005 /$ Reconstruction $0.1 \%$ (0.5\% max)

'1-H-1 FROM ENDF/B-VI '/

'PROCESSED BY NJOY-99 '/

0 /

broadr / Doppler broadening to Unit-23

$-21-22-23$

$1251000 /$

$0.001 /$

300./

$0 /$

unresr / Does not do any harm if no unresolved res

$-21-23-24$

125141

300.

1.E10 1.E6 1.E3 10.

0 /

groupr

$\begin{array}{llll}-21 & -260 & 25\end{array}$

1251111141

'1-H-1 FROM ENDF/B-VI' /

300.

1.E10 1.E6 1.E3 10.

79/ number of neutron groups

1.0000E-05 5.0000E-04 2.0000E-03 5.0000E-03 1.0000E-02 1.4500E-02

2.1000E-02 3.0000E-02 4.0000E-02 5.0000E-02 7.0000E-02 1.0000E-01

$1.2500 \mathrm{E}-01$ 1.5000E-01 1.8400E-01 2.2500E-01 2.7500E-01 3.2500E-01

3.6680E-01 4.1399E-01 5.0000E-01 5.3158E-01 6.2506E-01 6.8256E-01

8.0000E-01 8.7643E-01 1.0000E+00 1.0400E+00 1.0800E+00 1.1253E+00

$1.3000 \mathrm{E}+00 \quad 1.4450 \mathrm{E}+00 \quad 1.8554 \mathrm{E}+00 \quad 2.3824 \mathrm{E}+00 \quad 3.0590 \mathrm{E}+00 \quad 3.9279 \mathrm{E}+00$

5.0435E+00 1.0677E+01 3.7266E+01 1.0130E+02 2.1445E+02 4.5400E+02

1.5846E+03 3.3546E+03 7.1017E+03 1.5034E+04 2.1875E+04 2.4176E+04 
2.6058E+04 3.1828E+04 4.0868E+04 6.7379E+04 1.1109E+05 1.8316E+05 $2.9721 \mathrm{E}+05$ 3.6883E+05 4.9787E+05 6.0810E+05 7.4274E+05 8.2085E+05 $1.0026 \mathrm{E}+06$ 1.3534E+06 1.6530E+06 1.9205E+06 2.2313E+06 2.3457E+06 $2.3653 \mathrm{E}+062.4660 \mathrm{E}+062.7253 \mathrm{E}+06$ 3.0119E+06 3.6788E+06 4.9659E+06 6.0653E+06 7.4082E+06 8.6071E+06 1.0000E+07 1.2214E+07 1.4191E+07 $1.7332 \mathrm{E}+071.9640 \mathrm{E}+07$

$399 /$ number of photon group

50000100000150000200000250000300000350000400000 450000500000550000600000650000700000750000800000 8500009000009500001000000105000011000001150000 1200000125000013000001350000140000014500001500000 1550000160000016500001700000175000018000001850000 1900000195000020000002050000210000021500002200000 2250000230000023500002400000245000025000002550000 2600000265000027000002750000280000028500002900000 2950000300000030500003100000315000032000003250000 3300000335000034000003450000350000035500003600000 3650000370000037500003800000385000039000003950000 4000000405000041000004150000420000042500004300000 4350000440000044500004500000455000046000004650000 4700000475000048000004850000490000049500005000000 5050000510000051500005200000525000053000005350000 5400000545000055000005550000560000056500005700000 $\begin{array}{llllll}5750000 & 58000005850000 & 5900000 & 5950000 & 6000000 & 6050000\end{array}$ 6100000615000062000006250000630000063500006400000 6450000650000065500006600000665000067000006750000 6800000685000069000006950000700000070500007100000 7150000720000072500007300000735000074000007450000 7500000755000076000007650000770000077500007800000 7850000790000079500008000000805000081000008150000 8200000825000083000008350000840000084500008500000 8550000860000086500008700000875000088000008850000 8900000895000090000009050000910000091500009200000 9250000930000093500009400000945000095000009550000 9600000965000097000009750000980000098500009900000 99500001000000010050000101000001015000010200000 $\begin{array}{lllllll}10250000 & 10300000 & 10350000 & 10400000 & 10450000 & 10500000\end{array}$ 105500001060000010650000107000001075000010800000 108500001090000010950000110000001105000011100000 111500001120000011250000113000001135000011400000 114500001150000011550000116000001165000011700000 117500001180000011850000119000001195000012000000 $\begin{array}{lllllll}12050000 & 12100000 & 12150000 & 12200000 & 12250000 & 12300000\end{array}$ $\begin{array}{lllllll}12350000 & 12400000 & 12450000 & 12500000 & 12550000 & 12600000\end{array}$ $\begin{array}{llllll}12650000 & 12700000 & 12750000 & 12800000 & 12850000 & 12900000\end{array}$ 129500001300000013050000131000001315000013200000 
132500001330000013350000134000001345000013500000

$\begin{array}{llllll}13550000 & 13600000 & 13650000 & 13700000 & 13750000 & 13800000\end{array}$

$\begin{array}{lllllll}13850000 & 13900000 & 13950000 & 14000000 & 14050000 & 14100000\end{array}$

$\begin{array}{llllll}14150000 & 14200000 & 14250000 & 14300000 & 14350000 & 14400000\end{array}$

144500001450000014550000146000001465000014700000

$\begin{array}{llllll}14750000 & 14800000 & 14850000 & 14900000 & 14950000 & 15000000\end{array}$

$\begin{array}{llllll}15050000 & 15100000 & 15150000 & 15200000 & 15250000 & 15300000\end{array}$

$\begin{array}{llllll}15350000 & 15400000 & 15450000 & 15500000 & 15550000 & 15600000\end{array}$

$\begin{array}{lllllll}15650000 & 15700000 & 15750000 & 15800000 & 15850000 & 15900000\end{array}$

$\begin{array}{lllllll}15950000 & 16000000 & 16050000 & 16100000 & 16150000 & 16200000\end{array}$

162500001630000016350000164000001645000016500000

$\begin{array}{lllllll}16550000 & 16600000 & 16650000 & 16700000 & 16750000 & 16800000\end{array}$

$\begin{array}{lllllll}16850000 & 16900000 & 16950000 & 17000000 & 17050000 & 17100000\end{array}$

$\begin{array}{llllll}17150000 & 17200000 & 17250000 & 17300000 & 17350000 & 17400000\end{array}$

$\begin{array}{llllll}17450000 & 17500000 & 17550000 & 17600000 & 17650000 & 17700000\end{array}$

$\begin{array}{llllll}17750000 & 17800000 & 17850000 & 17900000 & 17950000 & 18000000\end{array}$

$\begin{array}{llllll}18050000 & 18100000 & 18150000 & 18200000 & 18250000 & 18300000\end{array}$

$\begin{array}{llllll}18350000 & 18400000 & 18450000 & 18500000 & 18550000 & 18600000\end{array}$

$\begin{array}{llllll}18650000 & 18700000 & 18750000 & 18800000 & 18850000 & 18900000\end{array}$

189500001900000019050000191000001915000019200000

192500001930000019350000194000001945000019500000

195500001960000019650000197000001975000019800000

19850000199000001995000020000000

$\begin{array}{llllll}0.0000 \mathrm{E}+0 & 0.000 \mathrm{E}+00 & 0 & 0 & 1 & 89\end{array}$

$89 \quad 5$

1.0000E-5 5.250E-04 9.0000E-3 3.550E-01 1.6000E-2 5.520E-01

$2.4000 \mathrm{E}-2 \quad 7.120 \mathrm{E}-01 \quad 2.9000 \mathrm{E}-2 \quad 7.850 \mathrm{E}-01 \quad 3.3000 \mathrm{E}-2 \quad 8.290 \mathrm{E}-01$

4.3000E-2 8.980E-01 5.0000E-2 9.180E-01 5.4000E-2 9.210E-01

5.9000E-2 9.180E-01 7.0000E-2 $8.920 \mathrm{E}-01 \quad 9.0000 \mathrm{E}-2 \quad 7.990 \mathrm{E}-01$

$1.1200 \mathrm{E}-1 \quad 6.860 \mathrm{E}-01 \quad 1.4000 \mathrm{E}-1 \quad 5.200 \mathrm{E}-01 \quad 1.7000 \mathrm{E}-1 \quad 3.830 \mathrm{E}-01$

$2.1000 \mathrm{E}-1 \quad 2.520 \mathrm{E}-01 \quad 3.0000 \mathrm{E}-1 \quad 1.080 \mathrm{E}-01 \quad 4.0000 \mathrm{E}-1 \quad 6.870 \mathrm{E}-02$

4.9000E-1 5.100E-02 5.7000E-1 4.370E-02 6.0000E-1 4.130E-02

$1.0000 \mathrm{E}+0 \quad 2.491 \mathrm{E}-02 \quad 4.0000 \mathrm{E}+0 \quad 6.786 \mathrm{E}-03 \quad 9.1180 \mathrm{E}+3 \quad 2.977 \mathrm{E}-06$

$2.0000 \mathrm{E}+4 \quad 1.413 \mathrm{E}-06 \quad 3.0700 \mathrm{E}+4 \quad 9.884 \mathrm{E}-07 \quad 6.0700 \mathrm{E}+4 \quad 5.814 \mathrm{E}-07$

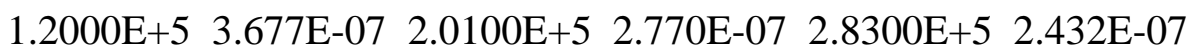

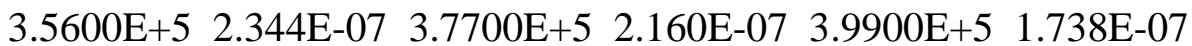

$\begin{array}{llllll}4.4200 \mathrm{E}+5 & 6.395 \mathrm{E}-08 & 4.7400 \mathrm{E}+5 & 1.381 \mathrm{E}-07 & 5.0200 \mathrm{E}+5 & 1.672 \mathrm{E}-07\end{array}$

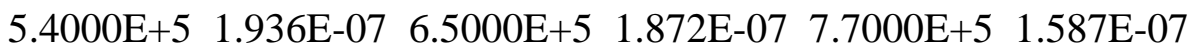

$9.0000 \mathrm{E}+5 \quad 1.363 \mathrm{E}-07 \quad 9.4100 \mathrm{E}+5 \quad 1.134 \mathrm{E}-07 \quad 1.0000 \mathrm{E}+6 \quad 7.268 \mathrm{E}-08$

$1.0500 \mathrm{E}+6 \quad 9.139 \mathrm{E}-08 \quad 1.1200 \mathrm{E}+6 \quad 1.083 \mathrm{E}-07 \quad 1.1900 \mathrm{E}+6 \quad 1.228 \mathrm{E}-07$

$\begin{array}{llllll}1.2100 \mathrm{E}+6 & 1.192 \mathrm{E}-07 & 1.3100 \mathrm{E}+6 & 5.451 \mathrm{E}-08 & 1.4000 \mathrm{E}+6 & 9.666 \mathrm{E}-08\end{array}$ $2.2200 \mathrm{E}+6 \quad 4.684 \mathrm{E}-08 \quad 2.3500 \mathrm{E}+6 \quad 5.814 \mathrm{E}-08 \quad 2.6300 \mathrm{E}+6 \quad 3.807 \mathrm{E}-08$

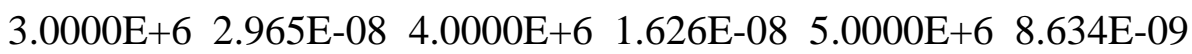
6.0000E+6 4.490E-09 $\quad 8.0000 \mathrm{E}+6 \quad 1.169 \mathrm{E}-09 \quad 1.0000 \mathrm{E}+7 \quad 2.947 \mathrm{E}-10$

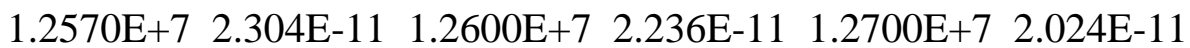
$1.2800 \mathrm{E}+7 \quad 1.832 \mathrm{E}-11 \quad 1.2900 \mathrm{E}+7 \quad 1.658 \mathrm{E}-11 \quad 1.3000 \mathrm{E}+7 \quad 1.501 \mathrm{E}-11$ 
$1.3100 \mathrm{E}+7 \quad 1.358 \mathrm{E}-11 \quad 1.3200 \mathrm{E}+7 \quad 1.229 \mathrm{E}-11 \quad 1.3300 \mathrm{E}+7 \quad 1.112 \mathrm{E}-11$ $\begin{array}{llllll}1.3400 \mathrm{E}+7 & 1.006 \mathrm{E}-11 & 1.3500 \mathrm{E}+7 & 9.108 \mathrm{E}-12 & 1.3600 \mathrm{E}+7 & 8.242 \mathrm{E}-12\end{array}$ $\begin{array}{llllll}1.3700 \mathrm{E}+7 & 7.458 \mathrm{E}-12 & 1.3800 \mathrm{E}+7 & 6.748 \mathrm{E}-12 & 1.3900 \mathrm{E}+7 & 6.106 \mathrm{E}-12\end{array}$ $1.4070 \mathrm{E}+7 \quad 5.151 \mathrm{E}-12 \quad 1.4200 \mathrm{E}+7 \quad 4.522 \mathrm{E}-12 \quad 1.4300 \mathrm{E}+7 \quad 4.091 \mathrm{E}-12$ $1.4400 \mathrm{E}+7 \quad 3.702 \mathrm{E}-12 \quad 1.4500 \mathrm{E}+7 \quad 3.349 \mathrm{E}-12 \quad 1.4600 \mathrm{E}+7 \quad 3.030 \mathrm{E}-12$ $\begin{array}{llllll}1.4700 \mathrm{E}+7 & 2.741 \mathrm{E}-12 & 1.4800 \mathrm{E}+7 & 2.479 \mathrm{E}-12 & 1.4900 \mathrm{E}+7 & 2.243 \mathrm{E}-12\end{array}$

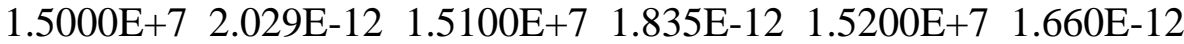

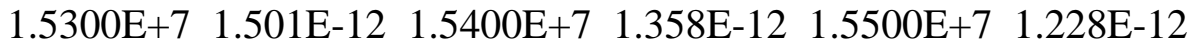
$1.5676 \mathrm{E}+7 \quad 1.029 \mathrm{E}-12 \quad 2.0000 \mathrm{E}+7 \quad 1.317 \mathrm{E}-14 /$

$16 /$

$0 /$

$0 /$

moder $-2327$ stop 


\section{APPENDIX H}

\section{Example RADSAT “Pseudo-Isotope” File for Phosphorus}

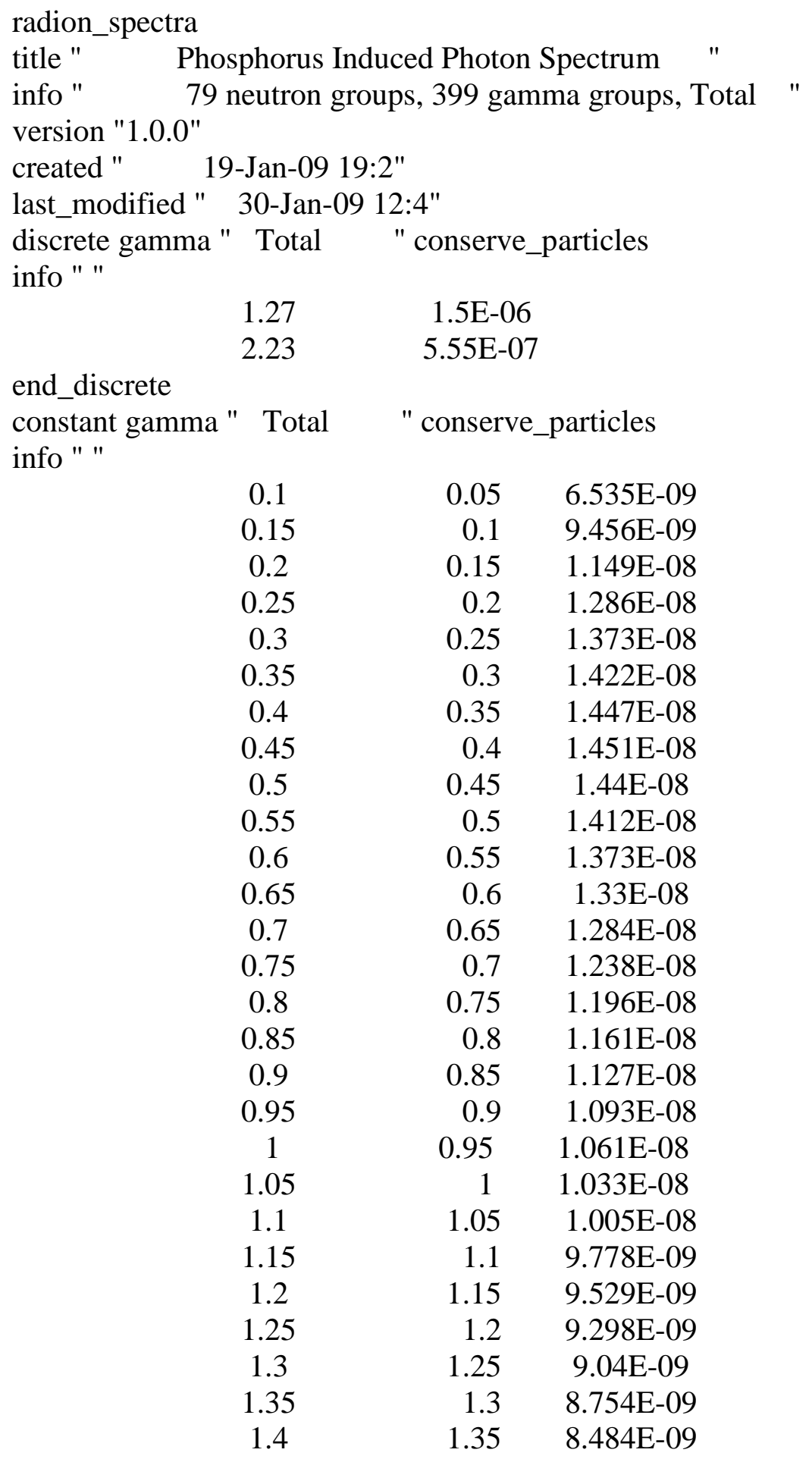




\begin{tabular}{|c|c|c|}
\hline 1.45 & 1.4 & $8.225 \mathrm{E}-09$ \\
\hline 1.5 & 1.45 & 7.97E-09 \\
\hline 1.55 & 1.5 & $7.508 \mathrm{E}-09$ \\
\hline 1.6 & 1.55 & 7.103E-09 \\
\hline 1.65 & 1.6 & $6.962 \mathrm{E}-09$ \\
\hline 1.7 & 1.65 & 6.819E-09 \\
\hline 1.75 & 1.7 & $6.671 \mathrm{E}-09$ \\
\hline 1.8 & 1.75 & $6.558 \mathrm{E}-09$ \\
\hline 1.85 & 1.8 & $6.479 \mathrm{E}-09$ \\
\hline 1.9 & 1.85 & $6.4 \mathrm{E}-09$ \\
\hline 1.95 & 1.9 & $6.321 \mathrm{E}-09$ \\
\hline 2 & 1.95 & $6.242 \mathrm{E}-09$ \\
\hline 2.05 & 2 & $6.076 \mathrm{E}-09$ \\
\hline 2.1 & 2.05 & $5.824 \mathrm{E}-09$ \\
\hline 2.15 & 2.1 & $5.572 \mathrm{E}-09$ \\
\hline 2.2 & 2.15 & $5.32 \mathrm{E}-09$ \\
\hline 2.25 & 2.2 & $5.068 \mathrm{E}-09$ \\
\hline 2.3 & 2.25 & $4.87 \mathrm{E}-09$ \\
\hline 2.35 & 2.3 & 4.731E-09 \\
\hline 2.4 & 2.35 & $4.599 \mathrm{E}-09$ \\
\hline 2.45 & 2.4 & $4.466 \mathrm{E}-09$ \\
\hline 2.5 & 2.45 & 4.333E-09 \\
\hline 2.55 & 2.5 & $4.195 \mathrm{E}-09$ \\
\hline 2.6 & 2.55 & $4.051 \mathrm{E}-09$ \\
\hline 2.65 & 2.6 & 3.914E-09 \\
\hline 2.7 & 2.65 & 3.784E-09 \\
\hline 2.75 & 2.7 & $3.654 \mathrm{E}-09$ \\
\hline 2.8 & 2.75 & 3.543E-09 \\
\hline 2.85 & 2.8 & $3.451 \mathrm{E}-09$ \\
\hline 2.9 & 2.85 & $3.36 \mathrm{E}-09$ \\
\hline 2.95 & 2.9 & $3.272 \mathrm{E}-09$ \\
\hline 3 & 2.95 & 3.19E-09 \\
\hline 3.05 & 3 & 3.097E-09 \\
\hline 3.1 & 3.05 & $2.991 \mathrm{E}-09$ \\
\hline 3.15 & 3.1 & $2.888 \mathrm{E}-09$ \\
\hline 3.2 & 3.15 & $2.791 \mathrm{E}-09$ \\
\hline 3.25 & 3.2 & $2.696 \mathrm{E}-09$ \\
\hline 3.3 & 3.25 & $2.623 \mathrm{E}-09$ \\
\hline 3.35 & 3.3 & $2.574 \mathrm{E}-09$ \\
\hline 3.4 & 3.35 & 2.53E-09 \\
\hline 3.45 & 3.4 & $2.489 \mathrm{E}-09$ \\
\hline 3.5 & 3.45 & $2.447 \mathrm{E}-09$ \\
\hline 3.55 & 3.5 & $2.403 \mathrm{E}-09$ \\
\hline 3.6 & 3.55 & 2.359E-09 \\
\hline 3.65 & 3.6 & $2.318 \mathrm{E}-09$ \\
\hline 3.7 & 3.65 & $2.279 \mathrm{E}-09$ \\
\hline
\end{tabular}




$\begin{array}{crc}3.75 & 3.7 & 2.24 \mathrm{E}-09 \\ 3.8 & 3.75 & 2.149 \mathrm{E}-09 \\ 3.85 & 3.8 & 2.005 \mathrm{E}-09 \\ 3.9 & 3.85 & 1.862 \mathrm{E}-09 \\ 3.95 & 3.9 & 1.719 \mathrm{E}-09 \\ 4 & 3.95 & 1.577 \mathrm{E}-09 \\ 4.05 & 4 & 1.491 \mathrm{E}-09 \\ 4.1 & 4.05 & 1.459 \mathrm{E}-09 \\ 4.15 & 4.1 & 1.429 \mathrm{E}-09 \\ 4.2 & 4.15 & 1.402 \mathrm{E}-09 \\ 4.25 & 4.2 & 1.376 \mathrm{E}-09 \\ 4.3 & 4.25 & 1.362 \mathrm{E}-09 \\ 4.35 & 4.3 & 1.361 \mathrm{E}-09 \\ 4.4 & 4.35 & 1.36 \mathrm{E}-09 \\ 4.45 & 4.4 & 1.362 \mathrm{E}-09 \\ 4.5 & 4.45 & 1.365 \mathrm{E}-09 \\ 4.55 & 4.5 & 1.315 \mathrm{E}-09 \\ 4.6 & 4.55 & 1.211 \mathrm{E}-09 \\ 4.65 & 4.6 & 1.108 \mathrm{E}-09 \\ 4.7 & 4.65 & 1.007 \mathrm{E}-09 \\ 4.75 & 4.7 & 9.071 \mathrm{E}-10 \\ 4.8 & 4.75 & 6.46 \mathrm{E}-10 \\ 4.85 & 4.8 & 4.314 \mathrm{E}-10 \\ 4.9 & 5.85 & 4.242 \mathrm{E}-10 \\ 4.95 & 4.9 & 4.171 \mathrm{E}-10 \\ 5 & 5.95 & 4.106 \mathrm{E}-10 \\ 5.05 & 5.7 & 2.112 \mathrm{E}-10 \\ 5.1 & 5.5 & 2.65 \\ 5.15 & 5.05 & 4.183 \mathrm{E}-10 \\ 5.2 & 5.1 & 4.254 \mathrm{E}-10 \\ 5.25 & 5.15 & 4.325 \mathrm{E}-10 \\ 5.3 & 5.2 & 4.396 \mathrm{E}-10 \\ 5.35 & 5.25 & 4.364 \mathrm{E}-10 \\ 5.4 & 5.3 & 4.231 \mathrm{E}-10 \\ 5.45 & 5.35 & 4.1 \mathrm{E}-10 \\ 5.5 & 5.4 & 3.969 \mathrm{E}-10 \\ 5.55 & 5.45 & 3.839 \mathrm{E}-10 \\ 5.6 & 3.681 \mathrm{E}-10 \\ 5.65 & 3.496 \mathrm{E}-10 \\ 5.7 & 3.317 \mathrm{E}-10 \\ 5.75 & 5.143 \mathrm{E}-10 \\ 5.8 & 569 \mathrm{E}-10 \\ 5.85 & 5.9 & \\ 5.95 & 5.10 \\ 6 & 5.10 \\ & 5.9 & \end{array}$




\begin{tabular}{|c|c|c|}
\hline 6.05 & 6 & $2.481 \mathrm{E}-10$ \\
\hline 6.1 & 6.05 & $2.452 \mathrm{E}-10$ \\
\hline 6.15 & 6.1 & $2.422 \mathrm{E}-10$ \\
\hline 6.2 & 6.15 & $2.393 \mathrm{E}-10$ \\
\hline 6.25 & 6.2 & $2.364 \mathrm{E}-10$ \\
\hline 6.3 & 6.25 & $2.298 \mathrm{E}-10$ \\
\hline 6.35 & 6.3 & $2.197 \mathrm{E}-10$ \\
\hline 6.4 & 6.35 & $2.1 \mathrm{E}-10$ \\
\hline 6.45 & 6.4 & $2.003 \mathrm{E}-10$ \\
\hline 6.5 & 6.45 & $1.906 \mathrm{E}-10$ \\
\hline 6.55 & 6.5 & $2.152 \mathrm{E}-10$ \\
\hline 6.6 & 6.55 & $2.74 \mathrm{E}-10$ \\
\hline 6.65 & 6.6 & $3.329 \mathrm{E}-10$ \\
\hline 6.7 & 6.65 & $3.917 \mathrm{E}-10$ \\
\hline 6.75 & 6.7 & $4.507 \mathrm{E}-10$ \\
\hline 6.8 & 6.75 & $4.454 \mathrm{E}-10$ \\
\hline 6.85 & 6.8 & $3.755 \mathrm{E}-10$ \\
\hline 6.9 & 6.85 & $3.057 \mathrm{E}-10$ \\
\hline 6.95 & 6.9 & $2.36 \mathrm{E}-10$ \\
\hline 7 & 6.95 & $1.665 \mathrm{E}-10$ \\
\hline 7.05 & 7 & $1.437 \mathrm{E}-10$ \\
\hline 7.1 & 7.05 & $1.676 \mathrm{E}-10$ \\
\hline 7.15 & 7.1 & $1.917 \mathrm{E}-10$ \\
\hline 7.2 & 7.15 & $2.159 \mathrm{E}-10$ \\
\hline 7.25 & 7.2 & $2.401 \mathrm{E}-10$ \\
\hline 7.3 & 7.25 & $2.361 \mathrm{E}-10$ \\
\hline 7.35 & 7.3 & $2.038 \mathrm{E}-10$ \\
\hline 7.4 & 7.35 & $1.717 \mathrm{E}-10$ \\
\hline 7.45 & 7.4 & $1.396 \mathrm{E}-10$ \\
\hline 7.5 & 7.45 & $1.076 \mathrm{E}-10$ \\
\hline 7.55 & 7.5 & $9.584 \mathrm{E}-11$ \\
\hline 7.6 & 7.55 & $6.725 \mathrm{E}-11$ \\
\hline 7.65 & 7.6 & $3.987 \mathrm{E}-11$ \\
\hline 7.7 & 7.65 & $5.065 \mathrm{E}-11$ \\
\hline 7.75 & 7.7 & $6.143 \mathrm{E}-11$ \\
\hline 7.8 & 7.75 & $5.902 \mathrm{E}-11$ \\
\hline 7.85 & 7.8 & 4.344E-11 \\
\hline 7.9 & 7.85 & $2.785 \mathrm{E}-11$ \\
\hline 7.95 & 7.9 & $1.535 \mathrm{E}-11$ \\
\hline 8 & 7.95 & $1.052 \mathrm{E}-11$ \\
\hline 8.05 & 8 & $1.029 \mathrm{E}-11$ \\
\hline 8.1 & 8.05 & $1.007 \mathrm{E}-11$ \\
\hline 8.15 & 8.1 & $9.85 \mathrm{E}-12$ \\
\hline 8.2 & 8.15 & $9.634 \mathrm{E}-12$ \\
\hline 8.25 & 8.2 & $9.428 \mathrm{E}-12$ \\
\hline 8.3 & 8.25 & $9.227 \mathrm{E}-12$ \\
\hline
\end{tabular}




$\begin{array}{crc}8.35 & 8.3 & 9.026 \mathrm{E}-12 \\ 8.4 & 8.35 & 8.825 \mathrm{E}-12 \\ 8.45 & 8.4 & 8.629 \mathrm{E}-12 \\ 8.5 & 8.45 & 8.444 \mathrm{E}-12 \\ 8.55 & 8.5 & 8.263 \mathrm{E}-12 \\ 8.6 & 8.55 & 8.082 \mathrm{E}-12 \\ 8.65 & 8.6 & 7.901 \mathrm{E}-12 \\ 8.7 & 8.65 & 7.724 \mathrm{E}-12 \\ 8.75 & 8.7 & 7.555 \mathrm{E}-12 \\ 8.8 & 8.75 & 7.393 \mathrm{E}-12 \\ 8.85 & 8.8 & 7.231 \mathrm{E}-12 \\ 8.9 & 8.85 & 7.068 \mathrm{E}-12 \\ 8.95 & 8.9 & 6.906 \mathrm{E}-12 \\ 9 & 8.95 & 6.753 \mathrm{E}-12 \\ 9.05 & 9 & 6.608 \mathrm{E}-12 \\ 9.1 & 9.05 & 6.463 \mathrm{E}-12 \\ 9.15 & 9.1 & 6.319 \mathrm{E}-12 \\ 9.2 & 9.15 & 6.175 \mathrm{E}-12 \\ 9.25 & 9.2 & 6.034 \mathrm{E}-12 \\ 9.3 & 9.25 & 5.901 \mathrm{E}-12 \\ 9.35 & 9.3 & 5.772 \mathrm{E}-12 \\ 9.4 & 9.35 & 5.644 \mathrm{E}-12 \\ 9.45 & 9.4 & 5.516 \mathrm{E}-12 \\ 9.5 & 9.45 & 5.388 \mathrm{E}-12 \\ 9.55 & 9.5 & 5.265 \mathrm{E}-12 \\ 9.6 & 9.55 & 5.149 \mathrm{E}-12 \\ 9.65 & 9.6 & 5.037 \mathrm{E}-12 \\ 9.7 & 9.65 & 4.924 \mathrm{E}-12 \\ 9.75 & 9.7 & 4.811 \mathrm{E}-12 \\ 9.8 & 9.75 & 4.698 \mathrm{E}-12 \\ 9.85 & 9.8 & 4.59 \mathrm{E}-12 \\ 9.9 & 9.85 & 4.488 \mathrm{E}-12 \\ 9.95 & 9.9 & 4.39 \mathrm{E}-12 \\ 10 & 9.95 & 4.291 \mathrm{E}-12 \\ 10.05 & 10 & 4.193 \mathrm{E}-12 \\ 10.1 & 10.05 & 4.095 \mathrm{E}-12 \\ 10.15 & 10.1 & 3.033 \mathrm{E}-12 \\ 10.2 & 10.2 & 1.064 \mathrm{E}-12 \\ 10.25 & 1.058 \mathrm{E}-13 \\ 10.3 & 1.032 \mathrm{E}-13 \\ 10.35 & 1.007 \mathrm{E}-13 \\ 10.4 & 9.83 \mathrm{E}-14 \\ 10.45 & 9.388 \mathrm{E}-14 \\ & & 9.122 \mathrm{E}-14 \\ 10.5 & & \end{array}$




\begin{tabular}{|c|c|c|}
\hline 10.65 & 10.6 & $8.686 \mathrm{E}-14$ \\
\hline 10.7 & 10.65 & $8.469 \mathrm{E}-14$ \\
\hline 10.75 & 10.7 & $8.26 \mathrm{E}-14$ \\
\hline 10.8 & 10.75 & $8.063 \mathrm{E}-14$ \\
\hline 10.85 & 10.8 & $7.867 \mathrm{E}-14$ \\
\hline 10.9 & 10.85 & $7.672 \mathrm{E}-14$ \\
\hline 10.95 & 10.9 & 7.477E-14 \\
\hline 11 & 10.95 & 7.293E-14 \\
\hline 11.05 & 11 & 7.119E-14 \\
\hline 11.1 & 11.05 & $6.945 \mathrm{E}-14$ \\
\hline 11.15 & 11.1 & $6.772 \mathrm{E}-14$ \\
\hline 11.2 & 11.15 & $6.6 \mathrm{E}-14$ \\
\hline 11.25 & 11.2 & $6.438 \mathrm{E}-14$ \\
\hline 11.3 & 11.25 & $6.283 \mathrm{E}-14$ \\
\hline 11.35 & 11.3 & $6.129 \mathrm{E}-14$ \\
\hline 11.4 & 11.35 & $5.975 \mathrm{E}-14$ \\
\hline 11.45 & 11.4 & $5.822 \mathrm{E}-14$ \\
\hline 11.5 & 11.45 & $5.679 \mathrm{E}-14$ \\
\hline 11.55 & 11.5 & $5.543 \mathrm{E}-14$ \\
\hline 11.6 & 11.55 & $5.406 \mathrm{E}-14$ \\
\hline 11.65 & 11.6 & $5.27 \mathrm{E}-14$ \\
\hline 11.7 & 11.65 & $5.134 \mathrm{E}-14$ \\
\hline 11.75 & 11.7 & $5.006 \mathrm{E}-14$ \\
\hline 11.8 & 11.75 & $4.886 \mathrm{E}-14$ \\
\hline 11.85 & 11.8 & $4.766 \mathrm{E}-14$ \\
\hline 11.9 & 11.85 & $4.647 \mathrm{E}-14$ \\
\hline 11.95 & 11.9 & $4.527 \mathrm{E}-14$ \\
\hline 12 & 11.95 & $4.412 \mathrm{E}-14$ \\
\hline 12.05 & 12 & 4.304E-14 \\
\hline 12.1 & 12.05 & $4.2 \mathrm{E}-14$ \\
\hline 12.15 & 12.1 & $4.095 \mathrm{E}-14$ \\
\hline 12.2 & 12.15 & $3.991 \mathrm{E}-14$ \\
\hline 12.25 & 12.2 & $3.886 \mathrm{E}-14$ \\
\hline 12.3 & 12.25 & $3.789 \mathrm{E}-14$ \\
\hline 12.35 & 12.3 & $3.698 \mathrm{E}-14$ \\
\hline 12.4 & 12.35 & $3.608 \mathrm{E}-14$ \\
\hline 12.45 & 12.4 & $3.517 \mathrm{E}-14$ \\
\hline 12.5 & 12.45 & $3.427 \mathrm{E}-14$ \\
\hline 12.55 & 12.5 & $3.336 \mathrm{E}-14$ \\
\hline 12.6 & 12.55 & $3.252 \mathrm{E}-14$ \\
\hline 12.65 & 12.6 & $3.174 \mathrm{E}-14$ \\
\hline 12.7 & 12.65 & $3.096 \mathrm{E}-14$ \\
\hline 12.75 & 12.7 & $3.018 \mathrm{E}-14$ \\
\hline 12.8 & 12.75 & $2.941 \mathrm{E}-14$ \\
\hline 12.85 & 12.8 & $2.863 \mathrm{E}-14$ \\
\hline 12.9 & 12.85 & $1.412 \mathrm{E}-14$ \\
\hline
\end{tabular}




\begin{tabular}{|c|c|}
\hline 12.95 & 12.9 \\
\hline 13 & 12.95 \\
\hline 13.05 & 13 \\
\hline 13.1 & 13.05 \\
\hline 13.15 & 13.1 \\
\hline 13.2 & 13.15 \\
\hline 13.25 & 13.2 \\
\hline 13.3 & 13.25 \\
\hline 13.35 & 13.3 \\
\hline 13.4 & 13.35 \\
\hline 13.45 & 13.4 \\
\hline 13.5 & 13.45 \\
\hline 13.55 & 13.5 \\
\hline 13.6 & 13.55 \\
\hline 13.65 & 13.6 \\
\hline 13.7 & 13.65 \\
\hline 13.75 & 13.7 \\
\hline 13.8 & 13.75 \\
\hline 13.85 & 13.8 \\
\hline 13.9 & 13.85 \\
\hline 13.95 & 13.9 \\
\hline 14 & 13.95 \\
\hline 14.05 & 14 \\
\hline 14.1 & 14.05 \\
\hline 14.15 & 14.1 \\
\hline 14.2 & 14.15 \\
\hline 14.25 & 14.2 \\
\hline 14.3 & 14.25 \\
\hline 14.35 & 14.3 \\
\hline 14.4 & 14.35 \\
\hline 14.45 & 14.4 \\
\hline 14.5 & 14.45 \\
\hline 14.55 & 14.5 \\
\hline 14.6 & 14.55 \\
\hline 14.65 & 14.6 \\
\hline 14.7 & 14.65 \\
\hline 14.75 & 14.7 \\
\hline 14.8 & 14.75 \\
\hline 14.85 & 14.8 \\
\hline 14.9 & 14.85 \\
\hline 14.95 & 14.9 \\
\hline 15 & 14.95 \\
\hline 15.05 & 15 \\
\hline 15.1 & 15.05 \\
\hline 15.15 & 15.1 \\
\hline 15.2 & 15.15 \\
\hline
\end{tabular}




\begin{tabular}{|c|c|}
\hline 15.25 & 15.2 \\
\hline 15.3 & 15.25 \\
\hline 15.35 & 15.3 \\
\hline 15.4 & 15.35 \\
\hline 15.45 & 15.4 \\
\hline 15.5 & 15.45 \\
\hline 15.55 & 15.5 \\
\hline 15.6 & 15.55 \\
\hline 15.65 & 15.6 \\
\hline 15.7 & 15.65 \\
\hline 15.75 & 15.7 \\
\hline 15.8 & 15.75 \\
\hline 15.85 & 15.8 \\
\hline 15.9 & 15.85 \\
\hline 15.95 & 15.9 \\
\hline 16 & 15.95 \\
\hline 16.05 & 16 \\
\hline 16.1 & 16.05 \\
\hline 16.15 & 16.1 \\
\hline 16.2 & 16.15 \\
\hline 16.25 & 16.2 \\
\hline 16.3 & 16.25 \\
\hline 16.35 & 16.3 \\
\hline 16.4 & 16.35 \\
\hline 16.45 & 16.4 \\
\hline 16.5 & 16.45 \\
\hline 16.55 & 16.5 \\
\hline 16.6 & 16.55 \\
\hline 16.65 & 16.6 \\
\hline 16.7 & 16.65 \\
\hline 16.75 & 16.7 \\
\hline 16.8 & 16.75 \\
\hline 16.85 & 16.8 \\
\hline 16.9 & 16.85 \\
\hline 16.95 & 16.9 \\
\hline 17 & 16.95 \\
\hline 17.05 & 17 \\
\hline 17.1 & 17.05 \\
\hline 17.15 & 17.1 \\
\hline 17.2 & 17.15 \\
\hline 17.25 & 17.2 \\
\hline 17.3 & 17.25 \\
\hline 17.35 & 17.3 \\
\hline 17.4 & 17.35 \\
\hline 17.45 & 17.4 \\
\hline 17.5 & 17.45 \\
\hline
\end{tabular}




\begin{tabular}{|c|c|}
\hline 17.55 & 17.5 \\
\hline 17.6 & 17.55 \\
\hline 17.65 & 17.6 \\
\hline 17.7 & 17.65 \\
\hline 17.75 & 17.7 \\
\hline 17.8 & 17.75 \\
\hline 17.85 & 17.8 \\
\hline 17.9 & 17.85 \\
\hline 17.95 & 17.9 \\
\hline 18 & 17.95 \\
\hline 18.05 & 18 \\
\hline 18.1 & 18.05 \\
\hline 18.15 & 18.1 \\
\hline 18.2 & 18.15 \\
\hline 18.25 & 18.2 \\
\hline 18.3 & 18.25 \\
\hline 18.35 & 18.3 \\
\hline 18.4 & 18.35 \\
\hline 18.45 & 18.4 \\
\hline 18.5 & 18.45 \\
\hline 18.55 & 18.5 \\
\hline 18.6 & 18.55 \\
\hline 18.65 & 18.6 \\
\hline 18.7 & 18.65 \\
\hline 18.75 & 18.7 \\
\hline 18.8 & 18.75 \\
\hline 18.85 & 18.8 \\
\hline 18.9 & 18.85 \\
\hline 18.95 & 18.9 \\
\hline 19 & 18.95 \\
\hline 19.05 & 19 \\
\hline 19.1 & 19.05 \\
\hline 19.15 & 19.1 \\
\hline 19.2 & 19.15 \\
\hline 19.25 & 19.2 \\
\hline 19.3 & 19.25 \\
\hline 19.35 & 19.3 \\
\hline 19.4 & 19.35 \\
\hline 19.45 & 19.4 \\
\hline 19.5 & 19.45 \\
\hline 19.55 & 19.5 \\
\hline 19.6 & 19.55 \\
\hline 19.65 & 19.6 \\
\hline 19.7 & 19.65 \\
\hline 19.75 & 19.7 \\
\hline 19.8 & 19.75 \\
\hline
\end{tabular}




$\begin{array}{lcrc} & 19.85 & 19.8 & 0 \\ & 19.9 & 19.85 & 0 \\ 19.95 & 19.9 & 0 \\ & 20 & 19.95 & 0 \\ \text { end_constant } & & & \\ \text { end_radion_spectra } & & \end{array}$




\section{REFERENCES}

1. L.E. SMITH, et al., "Coupling Deterministic and Monte Carlo Transport Methods for the Simulation of Gamma-Ray Spectroscopy Scenarios," submitted to IEEE Trans. Nucl. Sci., December 2008.

2. MCNP-A General Monte Carlo N-Particle Transport Code, Los Alamos National Laboratory, LA-UR-03-1987, 2003.

3. NJOY99.0 - Code System for Producing Pointwise and Multigroup Neutron and Photon Cross-Sections from ENDF/B Data, Oak Ridge National Laboratory, PSR480/NJOY99.0 (2000).

4. G.W. McKinney, "MCNP Applications for the $21^{\text {st }}$ Century," SNA 2000 Conference, Tokyo, Japan, September 2000.

5. $M C N P X^{T M}$ User's Manual, Los Alamos National Laboratory, LA-UR-02-2607, 2002.

6. S. Agostinelli, J. Allison, K. Amako, J. Apostolakis, H. Araujo, P. Arce, et al., “GEANT4 - A Simulation Toolkit," Nucl. Inst. Meth. A, 506, 250-303, 2003.

7. Fluka: a multi-particle transport code, CERN European Organization for Nuclear Research, CERN-2005-010, 2005.

8. The MARS Code System User's Guide Version 15, Fermi National Accelerator Laboratory, 2009.

9. PENELOPE-2006: A Code System for Monte Carlo Simulation of Electron and Photon Transport, Workshop Proceedings Barcelona Spain, ISBN 92-64-023011,2006

10. J.J. Duderstadt and W.R. Martin, "Transport Theory,” John Wiley \& Sons, Inc., New York, 1979.

11. J.M. McGhee, et al., Attila User's Manual, Transpire, Inc. (2007).

12. W.A. Rhoades and D.B. Simpson, "The TORT Three-Dimensional Discrete Ordinates Neutron/Photon Transport Code," Oak Ridge National Laboratory, ORNL/TM-13221, 1997.

13. R.E. Alcouffe, R.S. Baker, J.A. Dahl, S.A. Turner, and R. Ward, "PARTISN: A Time-Dependent, Parallel Neutral Particle Transport Code System," Los Alamos 
National Laboratory, LA-UR-05-3925, 2005.

14. R.E. Alcouffe, R.S. Baker, F.W. Brinkley, D.R. Marr, R.D. O'Dell, and W.F. Walters, "DANTSYS: A Diffusion Accelerated Neutral Particle Transport Code System," Los Alamos National Laboratory, LA-12969-M, 1995.

15. T.A. Brunner, "Forms of Approximate Radiation Transport," SAND2002-1778, Sandia National Laboratory, 2002.

16. T.A. Wareing, J.M. McGhee, and J.E. Morel, "ATTILA, A Three-Dimensional, Unstructured Tetrahedral Mesh Discrete Ordinates Code,” Trans. Am. Nucl. Soc., 75, 146, 1996.

17. D.J. Mitchell and J. Mattingly, "Rapid Computation of Gamma-ray Spectra for One-Dimensional Source Models," American Nuclear Society Annual Meeting, San Diego, 2008.

18. I. Davis, (private communication), 2008.

19. M.W. Shaver, L.E. Smith, R.T. Pagh, E.A. Miller, and R.S. Wittman, "The Coupling of a Deterministic Transport Field Solution to a Monte Carlo Boundary Condition for the simulation of Large Gamma-Ray Spectrometers," submitted to Nuclear Technology, May 2008.

20. SCALE - Version 5.1, Oak Ridge National Laboratory, ORNL/TM-2005/39, 2006.

21. E.S. Varley and J. Mattingly, "Rapid Feynman-Y Synthesis: Kynea3 CrossSection Library Development," Trans. Amer. Nucl. Soc., Vol. 98, pp.575 - 576 (2008).

22. J. E. White, et al., "VITAMIN-B6: A Fine-Group Cross-Section Library Based on ENDF/B-VI for Radiation Transport Applications," Proc. of the International Conference on Nuclear Data for Science and Technology, Gatlinburg, TN, pp. 733-736 (1994).

23. J.E. White, et al., "BUGLE-96: A Revised Multigroup Cross-Section Library for LWR Applications Based on ENDF/B-VI Release 3," presented at the American Nuclear Society Radiation Protection \& Shielding Topical Meeting, Falmouth, MA (1996).

24. "SolidWorks" <http://www.solidworks.com/> (Accessed May 13, 2008).

25. T.A. Wareing, J.M. McGhee, J.E. Morel, S.D. Pautz, "Discontinuous Finite Element Sn Methods on Three-Dimensional Unstructured Grids," Nucl. Sci. 
Engr., Vol. 138, Number 2, July 2001.

26. T.A. Wareing, J.E. Morel, J.M. McGhee, "Coupled Electron-Photon Transport Methods on 3-D Unstructured Grids," Trans Am. Nucl. Soc., Washington D.C., Vol 83, 2000.

27. J.J. Duderstadt and L.J. Hamilton, "Nuclear Reactor Analysis," John Wiley \& Sons, Inc., New York, 1976.

28. I.I. Bondarenko, Ed., "Group Constants for Nuclear Reactor Calculations," Consultants Bureau, New York, 1964.

29. N.M. Greene, "BONAMI: Resonance Self-Shielding by the Bondarenko Method," Ridge National Laboratory, ORNL/NUREG/CSD-2/V2/R6, 1998.

30. L.J. Lorence, “CEPXS/ONELD Version 2.0: A Discrete Ordinates Code Package for General One-Dimensional Coupled Electron-Photon Transport," IEEE Trans. Nucl. Sci., 39, 1031, 1992.

31. Kenneth Barbalace. Periodic Table of the Elements.

EnvironmentalChemistry.com. 1995 - 2009. Accessed on-line: 4/22/2009

http://EnvironmentalChemistry.com/yogi/periodic/ 